BRIT S. SCHNEIDER

\title{
GESUNDHEIT UND BILDUNG
}

Theorie und Empirie der

Humankapitalinvestitionen 


\section{BRIT S. SCHNEIDER}

\section{GESUNDHEIT UND BILDUNG}

Demographische Alterung und technischer Fortschritt sind maßgebliche Ursachen für steigende Ausgaben im Gesundheitswesen. Reaktionen hierauf sind zusehends Beitragssatzsteigerungen und Kostendämpfungsmaßnahmen, weshalb der Markt für Gesundheitsgüter seine Wachstumspotenziale nicht voll auszuschöpfen vermag. In dieser Arbeit werden die positiven Aspekte der Gesundheit für die wirtschaftliche Entwicklung herausgearbeitet. Darüber hinaus wird das Zusammenspiel von Gesundheit und Bildung analysiert, da Bildung die Rolle eines zentralen Wachstumsmotors zukommt. Eine empirische Untersuchung gibt zudem Aufschluss über die wesentlichen Determinanten eines guten Gesundheitsverhaltens sowie einer guten Gesundheit als Voraussetzung für stetiges Wirtschaftswachstum.

Brit S. Schneider wurde 1978 in Hamburg geboren und studierte von 1997 bis 2001 Betriebswirtschaftslehre an der Universität Greifswald. Seit 2002 ist sie Wissenschaftliche Mitarbeiterin am Lehrstuhl für Volkswirtschaftslehre III, insbesondere Finanzwissenschaft der Universität Bayreuth. Die Promotion wurde 2006 abgeschlossen. 


\section{Gesundheit und Bildung}




\section{ALLOKATION IM MARKTWIRTSCHAFTLICHEN SYSTEM}

Herausgegeben von

Heinz König ( $\dagger$ ), Hans-Heinrich Nachtkamp,

Ulrich Schlieper, Eberhard Wille

\section{Band 54}

\section{(i.)

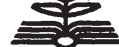 \\ PETER LANG}

Frankfurt am Main · Berlin - Bern - Bruxelles - New York · Oxford · Wien 


\section{BRIT S. SCHNEIDER}

\section{GESUNDHEIT \\ UND BILDUNG \\ Theorie und Empirie der Humankapitalinvestitionen}

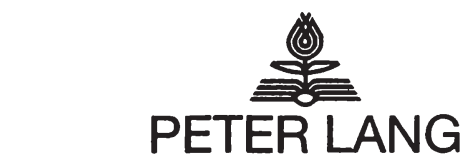

Europäischer Verlag der Wissenschaften

Brit S. Schneider - 978-3-631-75570-9

Downloaded from PubFactory at 01/11/2019 03:28:00AM

via free access 
Bibllografische Information der Deutschen Nationalbibliothek Die Deutsche Nationalbibliothek verzeichnet diese Publikation in der Deutschen Nationalbibliografie; detaillierte bibliografische Daten sind im Internet über <http://www.d-nb.de> abrufbar.

Zugl.: Bayreuth, Univ., Diss., 2006

Open Access: The online version of this publication is published on www.peterlang.com and www.econstor.eu under the international Creative Commons License CC-BY 4.0. Learn more on how you can use and share this work: http://creativecommons.org/licenses/ by/4.0.

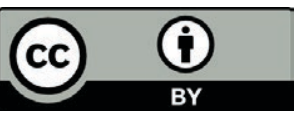

This book is available Open Access thanks to the kind support of ZBW - Leibniz-Informationszentrum Wirtschaft.

Gedruckt auf alterungsbeständigem, säurefreiem Papier.

\author{
D 703 \\ ISSN 0939-7728 \\ ISBN-10: 3-631-56248-9 \\ ISBN-13: 978-3-631-56248-2 \\ ISBN 978-3-631-75570-9 (eBook) \\ (c) Peter Lang GmbH \\ Europäischer Verlag der Wissenschaften \\ Frankfurt am Main 2007 \\ Alle Rechte vorbehalten.
}

Das Werk einschließlich aller seiner Teile ist urheberrechtlich geschützt. Jede Verwertung außerhalb der engen Grenzen des Urheberrechtsgesetzes ist ohne Zustimmung des Verlages unzulässig und strafbar. Das gilt insbesondere für Vervielfältigungen, Übersetzungen, Mikroverfilmungen und die Einspeicherung und Verarbeitung in elektronischen Systemen.

Printed in Germany 123457 www.peterlang.de 


\section{Vorwort}

Die öffentliche Diskussion über das deutsche Gesundheitswesen konzentriert sich insbesondere auf die Frage der Finanzierung sowie notwendige Einsparungen im Leistungskatalog. Medizinisch-technischer Fortschritt und demographische Alterung führen gegenwärtig zu immer weiter steigenden Beitragssätzen und somit zu einem scheinbar unaufhaltsamen Anstieg der Lohnnebenkosten. Auf der anderen Seite ist aber gerade die Gesundheit der Bevölkerung unabdingbar für stetiges Wirtschaftswachstum. Darüber hinaus stellt gerade das Gesundheitswesen einen dynamischen Wachstumsmarkt dar.

Die vorliegende Arbeit beschäftigt sich mit der Frage, welche direkten Wachstumseffekte von einer guten Gesundheit ausgehen. Der wohl noch bedeutendere Wachstumsmotor ist jedoch die Bildung, da insbesondere Forschung und Entwicklung die wesentlichen Wachstumsimpulse liefert. Hieran anknüpfend, werden auch die indirekten Wachstumseffekte einer guten Gesundheit analysiert, indem das wechselseitige Zusammenspiel zwischen Gesundheit und Bildung untersucht wird.

Diese Dissertation wurde im Sommersemester 2006 an der Rechts- und Wirtschaftswissenschaftlichen Fakultät der Universität Bayreuth angenommen. Sie entstand während meiner Tätigkeit als wissenschaftliche Mitarbeiterin am Lehrstuhl für Volkswirtschaftslehre, insbesondere Finanzwissenschaft, der Universität Bayreuth.

Ar dieser Stelle möchte ich mich bei all denen bedanken, die wesentlich zum Gelingen dieser Arbeit beigetragen haben. Mein besonderer Dank gilt meinem Doktorvater Herrn Prof. Dr. Volker Ulrich, der mich zum Verfassen dieser Arbeit ermutigt und mich stets darin bestärkt hat, meine Ideen im Rahmen dieser Arbeit umzusetzen. Weiter möchte ich Herrn Prof. Dr. Dr. h. c. Peter Oberender für die Übernahme des Zweitgutachtens sowie Herrn Prof. Dr. Martin Leschke für den Vorsitz des Prüfungsausschusses danken. Für wertvolle Hinweise und Anregungen danke ich zudem Herrn Dipl.-Volkswirt Timo Meidenbauer sowie Herrn cand. rer. pol. Oliver Gloede.

Zuletzt möchte ich mich ganz besonders bei meinem Mann Dr. Udo Schneider bedanken, der es immer wieder geschafft hat, mich zu motivieren und mich stets auf wundervolle Weise unterstützt hat.

Bayreuth, im Dezember 2006 
Brit S. Schneider - 978-3-631-75570-9

Downloaded from PubFactory at 01/11/2019 03:28:00AM

via free access 


\section{Inhaltsverzeichnis}

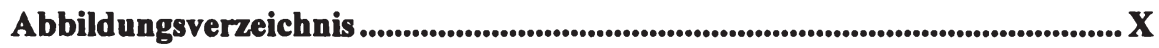

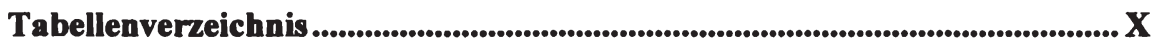

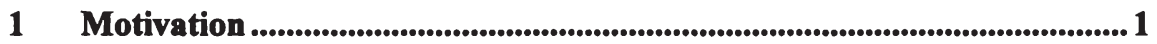

1.1 Gesundheit und die wirtschaftliche Entwicklung.................................... 1

1.2 Aufbau der Arbeit ........................................................................... 4

2 Der Begriff Humankapital ...............................................................................9 9

2.1 Humankapital in Form von Bildung.................................................... 9

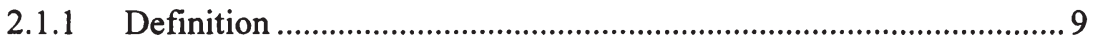

2.1.2 Messbarkeit ......................................................................... 11

2.1.3 Investitionen in das Bildungskapital ........................................... 12

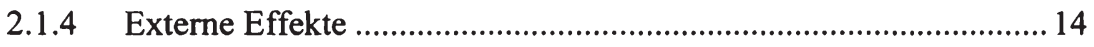

2.2 Gesundheit als Humankapital .......................................................... 15

2.2.1 Definitionsansätze .................................................................... 15

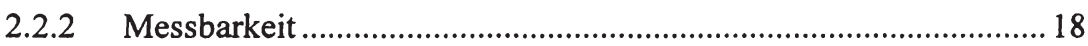

2.2.3 Gesundheit als Investitionsgut ...................................................... 19

2.2.4 Externe Effekte im Gesundheitswesen .......................................22

2.3 Unterschiede zwischen den Humankapitalarten .................................. 23

3 Humankapital als Wachstumsfaktor .........................................................27

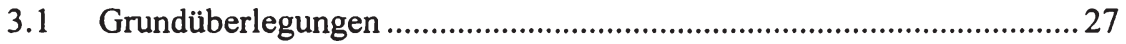

3.2 Die Bedeutung der Bildung für das Wirtschaftswachstum .................. 28

3.2.1 Wachstumstheoretische Ansätze .................................................28

3.2.2 Empirische Überprüfung des Bildungskapitalansatzes .................. 36

3.3 Gesundheit als Produktionsfaktor ....................................................... 42

3.3.1 Grundlegende Zusammenhänge ...................................................4 42

3.3.2 Wachstumstheoretische Modellansätze ...................................... 45

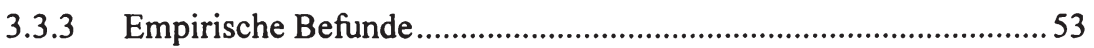

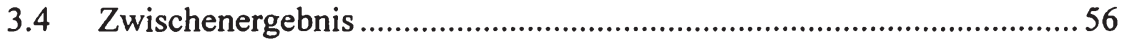




\section{Investitionen in die Gesundheit - Das Grossman-Modell}

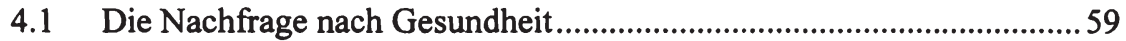

4.2 Der optimale Gesundheitskapitalstock ..............................................60

4.3 Modellkritik und Erweiterungen des Grossman-Modells ......................62

4.4 Zur Bedeutung von Einkommen und Bildung für die Nachfrage

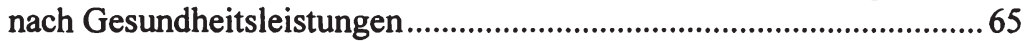

4.4.1 Gesundheitsrelevantes Konsumverhalten ....................................65

4.4.2 Die Bedeutung der Bildung für die Gesundheit .............................68

4.4.3 Zugang zu Gesundheitsleistungen ................................................ 70

5 Gesundheit und Arbeitsangebot ........................................................................ 73

5.1 Die Bedeutung der Arbeit für Gesundheitsinvestitionen ..................... 73

5.1.1 Grundüberlegungen......................................................................... 73

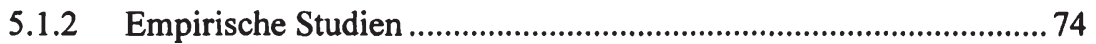

5.2 Der Zusammenhang zwischen medizinischen Leistungen und

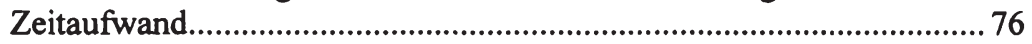

5.3 Die optimale Gesundheit in der kurzen Frist......................................... 78

5.3.1 Arbeit und Gesundheitsinvestitionen............................................ 82

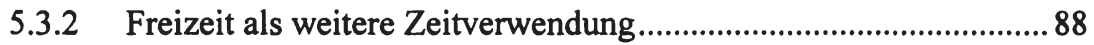

5.3.3 Komparativ-statische Analyse ......................................................92

5.4 Konsequenzen für das Arbeitsangebot in der langen Frist....................96

5.5 Zusammenfassung und Ausblick........................................................ 101

6 Grundlegende Zusammenhănge zwischen Gesundheit und

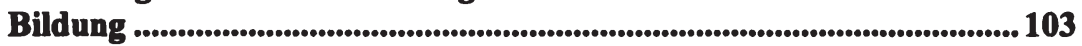

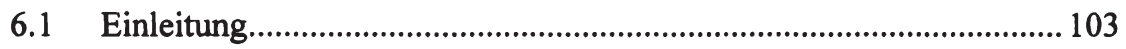

6.2 Gesundheitsorientierte Determinanten von Bildungsinvestitionen..... 108

6.2.1 Auswirkungen der Lebenserwartung auf die Bildungsentscheidung

6.2.2 Die Bedeutung von Unsicherheit und Versicherung für die Bildungsinvestitionen

6.3 Der Einfluss der Gesundheit auf die Produktivität der Bildungsinvestitionen 


\section{Ein theoretisches Modell zum Einfluss der Gesundheit auf die Bildung .............................................................................................................................. 123}

7.1 Die optimale Bildungsentscheidung unter Sicherheit ........................ 123

7.2 Investitionen unter Unsicherheit ohne Krankenversicherungsschutz.

7.3 Einführung einer prämienfinanzierten Krankenversicherung............. 140

7.4 Einführung einer steuerfinanzierten Krankenversicherung................ 149

7.4.1 Finanzierung über eine proportionale Lohnsteuer ....................... 149

7.4.2 Vergleich der Versicherungssysteme ........................................... 155

7.4.3 Erweiterung der Bemessungsgrundlage um Zinseinkünfte .......... 162

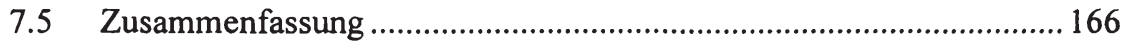

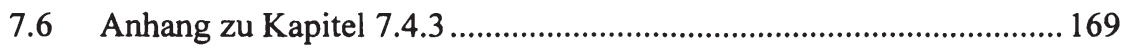

8 Empirische Analyse der Gesundheitsdeterminanten anhand von Mikrodaten .............................................................................................................. 170

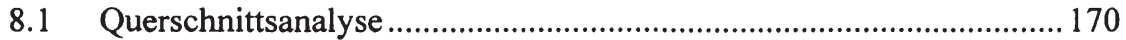

8.1.1 Datenmaterial und Hypothesen............................................... 171

8.1.2 Variablenbeschreibung ........................................................... 173

8.1.3 Deskriptive Statistik ..................................................................... 181

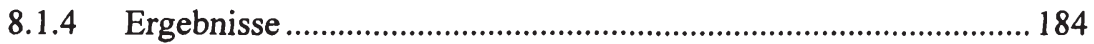

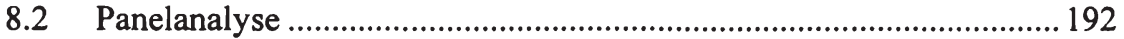

8.2.1 Vorteile und Probleme der Panelschätzung................................. 192

8.2.2 Deskriptive Statistik................................................................. 194

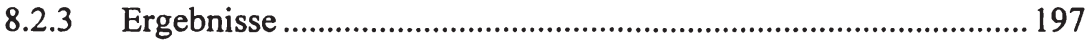

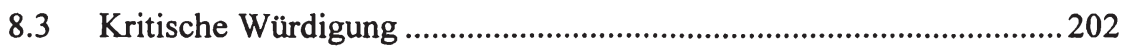

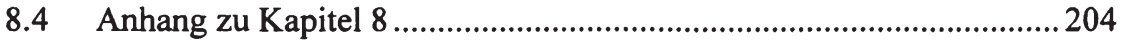

9 Zusammenfassung und Politikimplikationen............................................219

Literaturverzeichnis ............................................................................................ 224 


\section{Abbildungsverzeichnis}

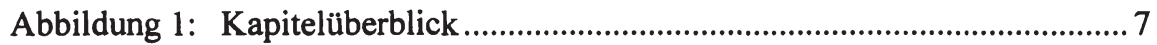

Abbildung 2: Bestimmungsfaktoren des Gesundheitszustandes.......................20

Abbildung 3: Bildungsbezogene Modellannahmen der Wachstumstheorie ..... 29

Abbildung 4: Gesundheitsbezogene Modellannahmen der Wachstumstheorie.

Abbildung 5: Schematische Darstellung der Zusammenhänge zwischen

Gesundheit, Bildung und Wirtschaftswachstum 57

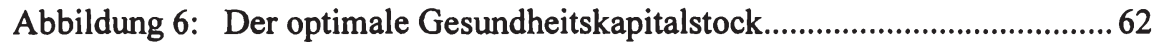

Abbildung 7: Gesundheit und Arbeitsangebot .......................................................93

Abbildung 8: Gesundheit und Arbeitszeit bei variablem Lohnsatz ................. 99

Abbildung 9: Determinanten der Bildungsinvestitionen................................. 104

Abbildung 10: Bildungsentscheidung und finanzieller Trade-off .................... 106

Abbildung 11: Finanzieller Trade-off unter Berücksichtigung von Krankheit.

Abbildung 12: Entgangene Einkommenszuwächse durch Krankheit................ 111

Abbildung 13: Lebenserwartung und Pro-Kopf-Einkommen........................... 116

Abbildung 14: Die optimale Bildungsinvestition unter Sicherheit ................... 127

Abbildung 15: Abnehmender Grenznutzen des Konsums ............................... 131

Abbildung 16: Investitionsentscheidung ohne Krankenversicherung............... 135

Abbildung 17: Bildungsinvestitionen bei prämienfinanziertem Krankenversicherungsschutz und unverändertem Konsumniveau.......... 145

Abbildung 18: Bildungsinvestitionen bei lohnsteuerfinanziertem Kranken-

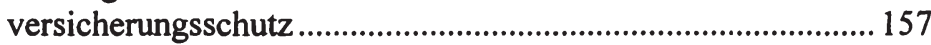

Abbildung 19: Normalverteilung und Logistische Verteilung..........................209

\section{Tabellenverzeichnis}

Tabelle 1: Die wichtigsten Gesundheitsdimensionen in der Health Insurance Study der Rand Corporation............................................. 17

Tabelle 2: Überblick über die Modellvariablen ..............................................8 84 
Tabelle 3: Wünschenswerter Body Mass Index im Verhältnis zum Alter .... 174

Tabelle 4: Verwendete Variablen in der Querschnittsanalyse........................ 175

Tabelle 5: Deskriptive Statistik, Querschnittsanalyse .................................. 182

Tabelle 6: Verteilung der 3 Gesundheitskategorien (in \%) .......................... 183

Tabelle 7: Anteil der Personen je Kategorie (Querschnitt)........................... 184

Tabelle 8: Ergebnisse der Querschnittsschätzung: gesundheitsrelevantes

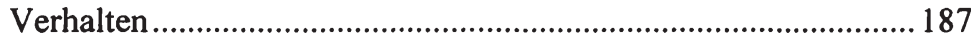

Tabelle 9: Ergebnisse der Querschnittsschätzung: eingeschätzter Gesund-

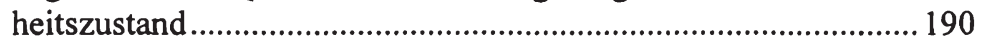

Tabelle 10: Verteilung der zwei Gesundheitskategorien (Panel) .................... 195

Tabelle 11: Anteil der Personen je Kategorie (Panel) ..................................... 196

Tabelle 12: Deskriptive Statistik der Panelanalyse......................................... 197

Tabelle 13: Ergebnisse der Panelschätzung: gesundheitsrelevantes Ver-

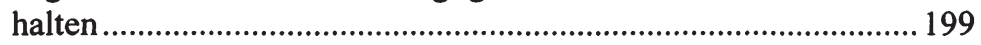

Tabelle 14: Ergebnisse der Panelschätzung: eingeschätzter Gesundheits-

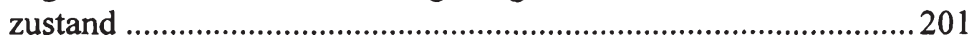


Brit S. Schneider - 978-3-631-75570-9

Downloaded from PubFactory at 01/11/2019 03:28:00AM

via free access 


\section{Motivation}

\subsection{Gesundheit und die wirtschaftliche Entwicklung}

Gesundheit und Wirtschaftswachstum - dies sind zwei Begriffe, die in der öffentlichen Meinung häufig nicht zusammenpassen. Die Entwicklung der Ausgaben für Gesundheitsgüter wird insgesamt sehr kritisch gesehen, da der hiermit verbundene hohe Finanzierungsbedarf im Gesundheitswesen zu hohen Lohnnebenkosten und damit zu einer zunehmenden Belastung des Faktors Arbeit führt. Die Zahlen, die das Bundesministerium für Gesundheit und Soziale Sicherung (2005) veröffentlicht, sprechen anscheinend für sich: Im Jahr 2003 wurden insgesamt knapp $240 \mathrm{Mrd}$. Euro für ärztliche Leistungen, Medikamente und übrige Gesundheitsgüter ausgegeben. Dies entspricht einem Anteil der Gesundheitsausgaben am nominalen Bruttoinlandsprodukt (BIP) von 11,3 Prozent. Pro Einwohner wurden damit im Jahr etwa 2900 Euro für Gesundheitszwecke aufgewendet. Im Vergleich hierzu betrugen die Gesundheitsausgaben im Jahr 1995 gerade 194,0 Mrd. Euro oder 10,8 Prozent des BIP. Die Gesundheitsausgaben pro Einwohner betrugen 1995 etwa 2380 Euro.

Eine ähnliche Entwicklung lässt sich ausmachen, wenn nur die Einnahmen- und Ausgabenentwicklung der gesetzlichen Krankenversicherung (GKV), die stets im Zentrum der politischen Diskussion steht, betrachtet wird. Zwar sind die Einnahmen von 1995 bis zum Jahr 2003 stetig gestiegen, dem stehen aber auch entsprechende Steigerungen auf der Ausgabenseite gegenüber. Insgesamt wurden im Zeitraum von 1995 bis 2003 1,196 Bio. Euro durch die gesetzlichen Kassen ausgegeben, aber nur 1,180 Bio. Euro an Einnahmen erzielt. Trotz der ständigen Bemühungen der jeweiligen Bundesregierung, die Ausgaben der Kassen durch diskretionäre Eingriffe und eine Stärkung der Selbstbeteiligung zu begrenzen, ist in dem betrachteten Jahrzehnt ein Defizit in Höhe von rund $16 \mathrm{Mrd}$. Euro entstanden. Gleichzeitig sind die Beitragssätze von durchschnittlich 13,20 Prozent (1995) auf 14,31 Prozent (2003) angestiegen.

Die Zahlen belegen eines der wesentlichen Probleme des deutschen Gesundheitswesens: Durch den medizinisch-technischen Fortschritt und das hiermit verbundene Problem der demographischen Alterung müssen die immer weiter steigenden Ausgaben durch eine immer geringer werdende Zahl Erwerbstätiger finanziert werden, weshalb allgemein von einer "Wachstumsschwäche der Finanzierungsbasis" (Sachverständigenrat für die Konzertierte Aktion im Gesundheitswesen (2003), S. 58) gesprochen wird. 'Die Orientierung der zu zahlenden

${ }^{1}$ Grundsätzlich gilt zwar eine Gleichbehandlung von Erwerbseinkommen und Renten, indem der Beitragssatz zur GKV auf beide Einkommensarten gleichermaßen erhoben wird. Dennoch 
Beiträge an den Erwerbseinkommen führt gleichzeitig dazu, dass Arbeit immer teurer wird, ohne dass dieser Entwicklung ein Produktivitätsanstieg zugrunde liegt. Die Folge hieraus ist steigende Arbeitslosigkeit. Aus dieser Argumentation könnte gefolgert werden, dass die Gesetzliche Krankenversicherung in seiner heutigen Form die Wachstumsrate des Einkommens negativ beeinflusst. ${ }^{2}$

Dennoch stellt gerade die Gesundheit einen zentralen Wachstumsfaktor dar. Das Gesundheitswesen bietet ein enormes Wachstumspotential, indem immer neue Technologien entwickelt werden. Gleichzeitig besteht insbesondere aufgrund der Alterung ein steigender Bedarf an Beschäftigten, sodass von einem „Wachstumsmarkt Gesundheit" (Oberender et al. (2002)) gesprochen werden kann. Nichtsdestotrotz muss eine solide Finanzierung dieser Entwicklung gewährleistet sein, damit knappe Ressourcen nicht nur umgeschichtet werden. Dies kann durch eine Stärkung der privaten Nachfrage nach Gesundheitsgütern oder durch eine Steigerung der Einnahmen geschehen.

Die vorliegende Arbeit befasst sich mit der Frage, welche direkten Wachstumseffekte mit der Gesundheit der Bevölkerung verbunden sind und wie sich hierdurch einige der genannten Probleme abbauen lassen. Zunächst bietet sich hier die Betrachtung der Arbeitskraft als Produktionsfaktor an. Um Arbeit gegenüber dem physischen Kapital wieder aufzuwerten, müssen sich sowohl die Quantität als auch die Qualität erhöhen. Hierzu sollte es ein Ziel sein, die Leistungen des Gesundheitswesens optimal zu nutzen, um die krank verbrachte Zeit der Individuen zu minimieren und so das vorhandene Arbeitskräftepotential auszuschöpfen. ${ }^{3}$

Eine dauerhafte Einkommenssteigerung und damit dauerhaftes Wirtschaftswachstum lassen sich darüber hinaus erzielen, wenn die Produktivität der Beschäftigten ansteigt. Hierfür ist es zum Ersten notwendig, in die Gesundheit zu investieren, denn nur gesunde Arbeitskräfte können die ihnen aufgetragenen Aufgaben schnell und zuverlässig ausführen. Zum Zweiten kann technischer

kommt es zu einer intergenerativen Umverteilung, da die bezogenen Renten im Durchschnitt geringer sind als die Erwerbseinkommen, weshalb Rentner absolut gesehen weniger Beiträge zahlen.

${ }^{2}$ Der Sachverständigenrat für die Konzertierte Aktion im Gesundheitswesen gibt weiterhin zu bedenken, dass die Entwicklung der beitragspflichtigen Einnahmen je Mitglied hinter dem Wachstum des BIP je Erwerbstätigen zurückbleibt, weshalb „,bei stabilen Beitragssătzen die Leistungen der GKV im Wachstum hinter der allgemeinen Wirtschaftsentwicklung zurückbleiben" (Sachverständigenrat für die Konzertierte Aktion im Gesundheitswesen (2003), Ziffer 54).

${ }^{3}$ Dies gilt im Übrigen nicht nur für Erwerbstătige, sondern für alle Personen, da der Konsum ebenfalls negativ durch Krankheit beeinflusst wird. 
Fortschritt jedoch nur bewusst generiert werden, wenn das in einer Ökonomie verfügbare Bildungskapital stetig zunimmt, da Bildung für die Erforschung und Anwendung neuer Technologien unabdingbar ist. Hierzu ist eine gute Gesundheit Grundvoraussetzung, da diese zum einen die Länge der Erwerbsperiode determiniert und so die Erträge von Bildungsinvestitionen nachhaltig beeinflusst, zum anderen aber auch die Lernfähigkeit bestimmt, sodass Investitionen in die Bildung schneller und mit höherer Produktivität durchgeführt werden können.

Da Krankheit oder Verletzungen nie vollständig ausgeschlossen werden können, ist zudem die Ausgestaltung eines Krankenversicherungssystems für die Bereitschaft zur Bildungskapitalakkumulation maßgeblich. Die Versicherung dient allgemein dazu, die finanziellen Belastungen im Krankheitsfall abzumildern, indem notwendige Behandlungen finanziert werden und ein Ersatz für entgangene Erwerbseinkommen geleistet wird. Zu diesem Zweck werden bei Gesundheit Beiträge oder Versicherungsprämien gezahlt, wodurch es zu einer Umverteilung von gesunden zu kranken Menschen kommt. Bei der Ausgestaltung eines solchen Versicherungssystems ist insbesondere darauf zu achten, dass sich dieses nicht negativ auf die Bildungsinvestitionen auswirkt. Dies könnte insbesondere dann der Fall sein, wenn Erwerbs- und übrige Einkommen unterschiedlich behandelt werden, so dass es zu einer finanziellen Benachteiligung des Humankapitals kommt.

Die hier genannten Punkte über die Finanzierung des Versicherungssystems betreffen sämtlich die Einnahmenseite der gesetzlichen Krankenversicherung, indem nicht nur die Erwerbstätigkeit in der Bevölkerung gesteigert wird, sondern darüber hinaus auch die Qualität der Arbeitskräfte ansteigt. Während der Gesundheit hier eine unterstützende Wirkung zukommt, ist Bildung als eigentlicher Wachstumsmotor zu betrachten, da Bildungskapital im Gegensatz zu Gesundheit unbegrenzt akkumuliert werden kann. ${ }^{4}$ Hierzu ist es allerdings notwendig, die Rahmenbedingungen derart zu setzen, dass die Bereitschaft zu mehr Bildung vorhanden ist und vorhandene Fähigkeiten auch entsprechend genutzt werden.

An diese Überlegungen knüpft schließlich die Frage an, unter welchen Bedingungen eine optimale Gesundheit erreicht werden kann, denn auch hier spielen die Rahmenbedingungen eine große Rolle. Zudem gehen mit einer Verbesserung

${ }^{4}$ Diese Annahme wird durch die Überlegung begründet, dass es keine theoretische Obergrenze für die Erfindung neuer Produkte und Herstellungsverfahren gibt, weshalb neues Wissen unbegrenzt generiert werden kann. Für die Gesundheit folgt hingegen, dass zumindest auf theoretischer Ebene ein Zustand vollkommener Gesundheit existiert. 
der Gesundheit nicht nur Produktivitätseffekte, sondern naturgemäß auch sinkende Behandlungskosten auf der Ausgabenseite einher. Somit kann sich eine Förderung gesundheitsrelevanten Verhaltens nicht nur positiv auf die Einkommen der betreffenden Individuen und damit auf das Wirtschaftswachstum auswirken, sondern es kommt gleichzeitig zu einer Senkung des Finanzierungsbedarfs. Hiermit ist wiederum eine geringere Belastung des verfügbaren Einkommens verbunden, woraus sich letztlich weitere Konsumeffekte ergeben können.

\subsection{Aufbau der Arbeit}

Der Aufbau der Arbeit gestaltet sich wie folgt: Im zweiten Kapitel werden Gesundheit und Bildung als Bestandteile des Humankapitals definiert und Gemeinsamkeiten sowie Unterschiede zwischen den Humankapitalarten, auch im Hinblick auf die empirische Verwertbarkeit, herausgearbeitet. Ziel dieses Kapitels soll es sein, die Betrachtung der menschlichen Leistungsfähigkeit als Kapitalgut zu motivieren. Es wird gezeigt, dass beide Kapitalarten, Gesundheit und Bildung, ähnlich dem physischen Kapital zwar Abschreibungen unterliegen, durch Investitionen aber ebenso auch akkumuliert werden können.

In der nachfolgenden Analyse des dritten Kapitels steht die Arbeitskraft als eigenständiger Produktionsfaktor im Vordergrund, wobei zunächst insbesondere das verfügbare Bildungskapital als Wachstumsfaktor betrachtet wird. Im ersten Abschnitt wird demnach die Frage behandelt, ob Bildung $\mathrm{zu}$ endogenem Wachstum führt und inwiefern sich Unterschiede in Wachstumsraten über Unterschiede in der Bildung erklären lassen. Zum Zweck einer verbesserten Übersichtlichkeit werden die Annahmen ausgewählter wachstumstheoretischer Modelle in funf Kategorien unterteilt und danach beurteilt, inwiefern diese Annahmen die neoklassische Wachstumstheorie oder die endogene Wachstumstheorie stützen. Die kontroverse Diskussion über die Rolle der Bildung zeigt sich zudem in einer Reihe empirischer Arbeiten, über die im Anschluss ein Überblick gegeben wird.

In Abschnitt 3.3 des gleichen Kapitels wird schließlich die Gesundheit als Determinante des Wirtschaftswachstums betrachtet. Hierzu werden zunächst grundlegende Zusammenhänge untersucht, wobei sowohl auf direkte Gesundheitseffekte als auch auf indirekte Effekte, die mit der Akkumulation von Bildungskapital zusammenhängen, Bezug genommen wird. Anschließend werden wiederum ausgewählte wachstumstheoretische Modelle anhand ihrer Annahmen klassifiziert und diskutiert. Ein Überblick über empirische Arbeiten, in denen beispielsweise die Lebenserwartung, die Kindersterblichkeit oder öffentliche Konsumausgaben für Gesundheitsgüter als Indikatoren für die Gesundheit per se herangezogen werden, erfolgt ebenfalls. Die Betrachtung der makroökonomi- 
schen Ebene dient als Motivation für die nachfolgenden Kapitel, in denen die Investitionen in die einzelnen Humankapitalbestandteile näher untersucht werden.

Hierzu wird in Kapitel 4 zunächst das Modell von Grossman (1970), in dem Individuen unter Aufwendung von Zeit und Einkommen Gesundheitsgüter nachfragen und so in ihre eigene Gesundheit investieren können, kurz beschrieben. Dieses Modell ist auch über 35 Jahre nach seinem Erscheinen noch aktuell und in seinen Annahmen zudem Grundlage einer eigenen Weiterentwicklung, die im anschließenden fünften Kapitel präsentiert wird. Somit werden insbesondere die Grundannahmen des Modells skizziert und kritisch diskutiert, wobei auch bereits bestehende Weiterentwicklungen des Modells kurz beschrieben werden.

Abschnitt 4.4 dieses Kapitels befasst sich mit der Nachfrage nach Gesundheitsleistungen, da diese für Investitionen in die Gesundheit unabdingbar sind. Hierzu wird zunächst die Rolle des Einkommens näher untersucht, das nicht nur den Konsum von originären Gesundheitsleistungen determiniert, sonder sich darüber hinaus auf die Ernährung sowie auf das häusliche Umfeld auswirkt. Im Anschluss werden Studien präsentiert, die sich mit dem Einfluss von Bildung auf die Gesundheit befassen. Dieser betrifft maßgeblich die Produktivität, mit der Gesundheitskapital akkumuliert werden kann. Den Schluss des vierten Kapitels bildet ein kurzer Überblick über mögliche Barrieren, welche den Zugang zu Gesundheitsleistungen beschränken und somit eine optimale Versorgung verhindern.

Das fünfte Kapitel behandelt mögliche Interdependenzen zwischen Arbeit und Gesundheit. Eine hohe Arbeitszeit impliziert auf der einen Seite ceteris paribus ein hohes Erwerbseinkommen, auf der anderen Seite wird hierdurch jedoch die für Gesundheitsinvestitionen verfügbare Zeit determiniert, so dass ein Optimierungsproblem in der Zeitallokation entsteht. $\mathrm{Zu}$ Beginn dieses Kapitels werden zunächst kurz die Kanäle, über die sich Arbeit auf die Gesundheit auswirken kann, beschrieben, indem sowohl theoretische Überlegungen als auch empirische Studien präsentiert werden.

Nach einem Überblick über mögliche Zusammenhänge zwischen der Nachfrage nach medizinischen Leistungen und dem mit Gesundheitsinvestitionen verbundenen Zeitaufwand erfolgt in Kapitel 5.3 eine Weiterentwicklung des GrossmanModells, indem nun insbesondere auf die Rolle der Arbeitszeit im Gesundheitsproduktionsprozess abgestellt wird. In dem Modell wird angenommen, dass die Gesundheit eine Funktion der eingesetzten Gesundheitsleistungen, der aufgewendeten Zeit sowie der Abschreibungen ist. In einem ersten Ansatz kann sich das betreffende Individuum lediglich zwischen Arbeitszeit und der Zeit für Ge- 
sundheitsinvestitionen entscheiden, von weiteren Zeitverwendungszwecken wird abstrahiert. In diesem Fall bedeutet eine Zunahme der Arbeitszeit einen Rückgang der Zeit für Gesundheitszwecke im gleichen Umfang. An dieses Ergebnis knüpft der folgende Abschnitt an, in dem Freizeit als weiterer Zeitverwendungszweck eingeführt wird. Aus den Arbeitszeiteffekten, die sich aus diesen Ansätzen ableiten lassen, ergeben sich schließlich langfristige Effekte, deren Schilderung in Kapitel 5.4 erfolgt.

Die wachstumstheoretische Analyse im dritten Kapitel hat ergeben, dass Gesundheit für die wirtschaftliche Entwicklung zwar unabdingbar ist, Bildung jedoch den eigentlichen Wachstumsmotor darstellt. Aus diesem Grund stellt sich über die Gesundheitsinvestitionen hinaus die Frage, von welchen Faktoren Investitionen in die Bildung abhängen. Im sechsten Kapitel wird daher der Einfluss der Gesundheit auf die Bildungsinvestitionen analysiert. Im Anschluss an eine einführende Darstellung der individuellen Bildungsentscheidung werden in Kapitel 6.2 gesundheitsorientierte Determinanten von Bildungsinvestitionen beschrieben. Hier kann gezeigt werden, wie sich Unsicherheit auf die Bereitschaft, Zeit für die Schul- sowie Ausbildung aufzuwenden, auswirkt, weshalb auch die Bedeutung von Versicherungen für die Bildungsinvestitionen kurz erörtert wird. Überlegungen zu möglichen Produktivitätseffekten einer guten Gesundheit beschließen dieses Kapitel.

Eine formale Darstellung zum Einfluss der Gesundheit auf die Bildung erfolgt in Kapitel 7. Zunächst wird ein Zwei-Perioden-Modell entwickelt, in dem von der Möglichkeit der Arbeitsunfähigkeit abstrahiert wird. In der ersten Periode können Individuen arbeiten oder aber einen Schul- oder höheren Bildungsabschluss machen. In der zweiten Periode ist das Individuum mit Sicherheit erwerbstätig, wobei sich das Erwerbseinkommen am erworbenen Abschluss ausrichtet. Dieses Szenario wird nachfolgend derart verändert, dass in der zweiten Periode ein positives Erkrankungsrisiko besteht. Zudem wird untersucht, inwiefern die Einführung einer Krankenversicherung nun dazu beitragen kann, die Unsicherheit über die Erträge der Bildungsinvestitionen zu mindern und so die Bereitschaft zu einer längeren Ausbildung zu fördern. In diesem Zusammenhang wird sowohl eine steuerfinanzierte Versicherung, ähnlich der deutschen GKV, als auch eine einkommensunabhängige Prämienfinanzierung im Sinne einer Pauschale untersucht.

Die ersten sieben Kapitel beschäftigen sich im Wesentlichen mit der Frage, auf welche Weise die Gesundheit der Bevölkerung das Wirtschaftswachstum positiv beeinflussen kann und welche Einflussfaktoren den individuellen Gesundheitszustand bedingen. Das achte Kapitel dient nun der empirischen Überprüfung der zuvor hergeleiteten theoretischen Ergebnisse. Hierzu wird zunächst das indivi- 
duelle Gesundheitsverhalten anhand der Indikatoren Tabakkonsum, Sport und Körpergewicht im Verhältnis zur Körpergröße untersucht. Von besonderem Interesse sind hierbei die Rolle der Bildung für ein gutes Gesundheitsverhalten, die Bedeutung des Einkommens sowie die Arbeitsbedingungen, gemessen an der Arbeitszeit. Darüber hinaus werden auch die Determinanten eines guten $\mathrm{Ge}-$ sundheitszustands empirisch bestimmt, da dieses für die Entscheidung, medizinische Leistungen in Anspruch zu nehmen, maßgeblich ist. Beide Fragestellungen werden sowohl im Rahmen einer Querschnittsschätzung als auch mittels einer Panelanalyse untersucht, wobei jeweils Mikrodaten des Sozioökonomischen Panels verwendet werden. Die ökonometrische Vorgehensweise wird im Anhang des achten Kapitels erläutert.

Die nachfolgende Abbildung 1 gibt einen grafischen Überblick über die Zusammenhänge zwischen den einzelnen Hauptbestandteilen dieser Arbeit. Anhand dieser Abbildung wird noch einmal deutlich, welche zahlreichen Interdependenzen zwischen den Faktoren Gesundheit, Bildung und Wirtschaftswachstum bestehen.

Abbildung 1: Kapitelüberblick

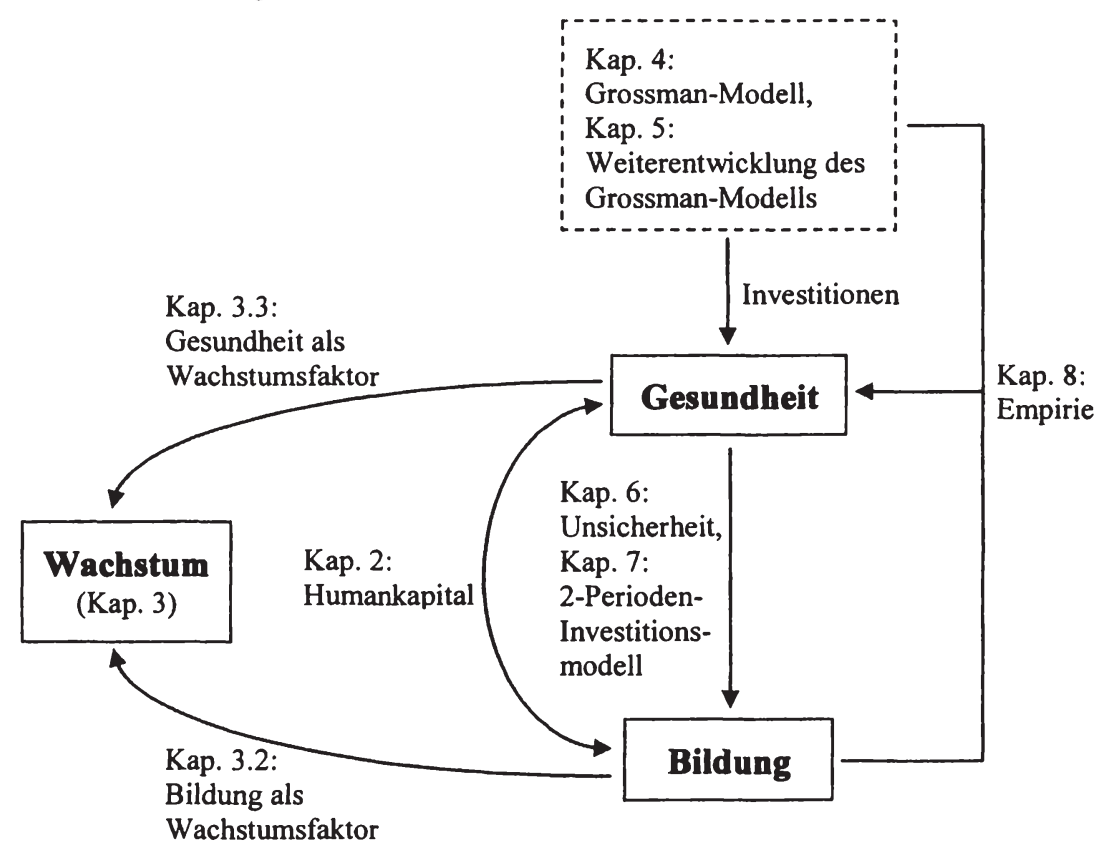

Quelle: Eigene Darstellung. 
Eine Zusammenfassung der wesentlichen Ergebnisse dieser Arbeit liefert abschließend das neunte Kapitel. Darüber hinaus werden in diesem letzten Kapitel Politikimplikationen formuliert, indem noch einmal anhand einiger Beispiele aufgezeigt wird, wo das deutsche Gesundheitswesen Ansatzpunkte für Verbesserungen bietet. 


\section{Der Begriff Humankapital}

Der Begriff „Humankapital“ spielt in zahlreichen Ansätzen der Wachstumstheorie (beispielsweise Nelson und Phelps (1966), Lucas (1988), Romer (1986) und Romer (1990)) eine zentrale Rolle. Das besondere Augenmerk dieser Arbeiten liegt jeweils auf der Bildung, die einen wesentlichen Bestandteil des Humankapitals darstellt. Diese wirkt sich direkt auf die Fähigkeiten und Fertigkeiten von Individuen aus und beeinflusst somit deren Produktivität positiv. Gleichwohl kann der Gesundheit ein ähnlicher Effekt auf die Effektivität der Arbeitskraft zugeschrieben werden, denn diese determiniert nicht nur die für Arbeit verfügbare Zeit, sondern sie ist zudem auch für die körperliche sowie geistige Leistungsfähigkeit maßgeblich. Insofern erscheint es sinnvoll, den Humankapitalbegriff weiter zu fassen und die Gesundheit zu integrieren. ${ }^{5}$

Eine Analyse der Literatur zeigt, dass Gesundheit und Bildung nicht nur jeweils als einzelne Komponenten des Humankapitals zu verstehen sind, vielmehr bedingen sie einander und sind somit direkt miteinander verknüpft. Damit kann das Humankapital als Produkt aus Bildungskapital auf der einen und Gesundheitskapital auf der anderen Seite verstanden werden.

\subsection{Humankapital in Form von Bildung}

\subsubsection{Definition}

Der Begriff „Bildungskapital“ ist sehr umfassend, weshalb zwischen einem Bildungsbegriff im engeren Sinne (i. e. S.) und einer Definition im weiteren Sinne (i. w. S.) unterschieden werden muss. Bildung i. e. S. umfasst die bewusste und planmäßige Erweiterung von Fähigkeiten sowie theoretischem Wissen mit Hilfe von Schul-, Aus- und Weiterbildung. Diese Art der Bildung dient der konsequenten Verbesserung der eigenen Wettbewerbsposition auf dem Arbeitsmarkt und damit der Steigerung von Erwerbseinkommen. Aufgrund eines zumeist gezielten Erwerbs von Bildungsnachweisen in Form von Zeugnissen und Zertifikaten ist der Bildungserfolg zumindest innerhalb eines bestehenden Bildungssystems relativ gut nachweisbar, auch wenn sich die Qualität einzelner gleichgestufter Bildungsinstitutionen unterscheiden kann. Die Begründung hierfür liegt

\footnotetext{
${ }^{5}$ In einem Ansatz von Schultz (1961) ist neben Gesundheit und Bildung auch Migration als Bestandteil des Humankapitals zu sehen, indem hierüber die eigenen Fähigkeiten mit den angebotenen Arbeitsplätzen in Einklang gebracht werden. Insofern steigert Migration den Wert der persönlichen Fähigkeiten, die sich aus Bildung und Gesundheit zusammensetzen. Von einem solchen Ansatz wird in der vorliegenden Arbeit jedoch abstrahiert, da Arbeit im Vergleich zu Kapital als weitgehend immobiler Faktor betrachtet wird.
} 
in der Annahme, dass sich Bildung immer aus dem Wissen vorangegangener Generationen zusammensetzt. Folglich ist der Bildungserfolg nicht nur durch die eigene Motivation bestimmt, sondern auch durch die Qualität der verwendeten Lehrmittel. Damit ist der Bildungsbegriff i. e. S. auf bereits bestehendes Wissen innerhalb der Gesellschaft beschränkt.

Bildungskapital i. w. S. umfasst neben der bewussten Bildung auch die unbewusste Akkumulation von Bildungskapital, die sich aus der Erfahrung, dem so genannten „Learning by Doing“, ergibt. Der Bildungsbegriff wird entsprechend um Fähigkeiten erweitert, die nicht dem originären Lernprozess zuzuordnen sind. Folglich wird nicht mehr nur bereits bestehendes Wissen reproduziert, sondern durch eigene Erfahrungswerte erweitert. Eine Konsequenz hieraus sind Innovationen, die sich aus eigenständiger Forschungstätigkeit ergeben. ${ }^{6}$ Solange neue Erkenntnisse der Allgemeinheit nicht zugänglich gemacht werden, handelt es sich hierbei um spezifisches Wissen, das auf einen kleinen Kreis von Individuen beschränkt ist und deren weitere Innovationstätigkeit begründet. Erst nach der Veröffentlichung von Forschungsergebnissen ist es möglich, auch den Bildungskapitalbestand i.e.S. zu erweitern, indem Innovationen zum Standard werden.

Zusätzlich zu der hier vorgenommenen Abgrenzung existiert in der Literatur die Unterscheidung zwischen spezifischem sowie unspezifischem Bildungskapital. ${ }^{8}$ Während vollkommen unspezifisches Wissen in allen Unternehmen gleichermaßen eingesetzt werden kann, ist absolut spezifisches Wissen an den jeweiligen Arbeitsplatz gekoppelt und stellt somit keinen Wettbewerbsvorteil auf dem Arbeitsmarkt dar. Für allgemeine Ausbildung gilt hingegen, dass diese nicht nur der ausbildenden Firma einen Produktivitätsvorteil verschafft, sondern dass dieser Vorteil in allen anderen Firmen ebenso bestehen würde. Finanziert ein Unternehmen die allgemeine Ausbildung in vollem Umfang, müssten sich diese direkten Ausbildungskosten negativ im zukünftigen Lohn des Auszubildenden niederschlagen, damit sich der Wert der Arbeit und die insgesamt entstandenen Kosten der Beschäftigung ausgleichen. Der Lohnsatz würde damit jedoch nicht mehr der zukünftigen Grenzproduktivität entsprechen und folglich unterhalb des

\footnotetext{
${ }^{6}$ Unter Innovationen werden technische oder organisatorische Neuerungen verstanden, die sich am Markt durchsetzen und damit ökonomisch verwertbar sind. Der Begriff der Invention beschreibt lediglich das Vorhandensein einer Erfindung, und zwar unabhängig von der tatsächlichen Nutzbarkeit. Aufgrund der fehlenden ökonomischen Relevanz wird nachfolgend nur die Innovationstătigkeit betrachtet.

${ }^{7}$ Grundsätzlich können Innovationen zwar durch Patente vor unmittelbarer Nachahmung geschutzt werden, eine Weiterentwicklung der Erfindung kann jedoch ebenso wenig verhindert werden wie die Produktion von Imitationen nach Ablauf des Patentschutzes.

${ }^{8}$ Siehe hierzu Becker (1993), Kapitel 3.
} 
Marktlohnsatzes liegen, wodurch ein Arbeitsplatzwechsel beschleunigt würde. Becker (1993) umschreibt diesen Zusammenhang folgendermaßen: „General training is useful in many firms besides those providing it." (S. 33).

Um eine solche Fehlentwicklung zu vermeiden, müssen die Ausbildungskosten zum Teil von den Auszubildenden selbst getragen werden. Dieser Eigenanteil ist umso größer, je allgemeiner die vermittelten Informationen und Fähigkeiten nutzbar sind. Die Bereitschaft für einen derartigen Einkommensverzicht ist bei den Individuen insbesondere dann gegeben, wenn die zukünftige Produktivität und damit das Lebenseinkommen durch das allgemeine Training gesteigert werden können. ${ }^{9}$

Spezifische Bildung umfasst alle Maßnahmen, von denen insbesondere das beschäftigende Unternehmen profitiert. Dieses Wissen ist im Extremfall auf dem Arbeitsmarkt wertlos, in der Regel ist jedoch zumindest der Produktivitätsanstieg in dem ausbildenden Unternehmen größer als in anderen Firmen am Markt. Die Finanzierung dieser Ausbildung hängt letztlich von der Wahrscheinlichkeit eines Jobwechsels ab. Wird die Ausbildung vollständig von dem Unternehmen finanziert und kommt es zu einer Kündigung durch den Beschäftigten, handelt es sich hierbei um für das Unternehmen verlorenes Kapital. Gleiches gilt für die Finanzierung durch einen Auszubildenden, dem gekündigt wird. Folglich muss eine längerfristige Bindung der Arbeitskraft an das Unternehmen gewährleistet sein. Die Wahrscheinlichkeit eines Arbeitsplatzwechsels kann effektiv eingedämmt werden, indem das Unternehmen nach Abschluss der Ausbildung einen Lohn zahlt, der über den übrigen Marktlöhnen liegt, so dass sich ein Wechsel finanziell nicht lohnt. Diese Bindung wird noch verstärkt, wenn ein Wechsel mit erneuten Investitionen in spezifisches Bildungskapital verbunden ist. Im Gegenzug tragen beide Seiten einen Teil der Ausbildungskosten, um die Anreize für die jeweils andere Seite, das Arbeitsverhältnis zu beenden, zu minimieren.

\subsubsection{Messbarkeit}

Die Messbarkeit von Bildungskapital hängt wesentlich von der verwendeten Definition ab. Beinhaltet der Begriff Bildung ausschließlich die Schul- und Ausbildung, so sind Dauer und Art der Ausbildung sowie die Abschlussnoten gute Indikatoren für den Bildungskapitalbestand. Hierbei handelt es sich um genormte Indikatoren, so dass eine direkte Vergleichbarkeit gegeben ist. Nicht direkt statistisch beobachtbar ist hingegen die Qualität der Ausbildung, da sich bei Schulen des gleichen Systems allein aufgrund der unterschiedlichen Lehrkräfte Diffe-

\footnotetext{
${ }^{9}$ In Deutschland ist eine solche Eigenbeteiligung der Auszubildenden durchweg gegeben, da die Einkommen in der Regel deutlich unter denen bereits ausgebildeter Arbeitskräfte liegen.
} 
renzen in dem vermittelten Lernstoff ergeben. Derartige Unterschiede lassen sich beispielsweise über Lese- und Mathematiktests offenbaren. ${ }^{10}$

Für das Humankapital als Produktionsfaktor ist nicht allein die Akkumulation von Bildungskapital von Interesse, vielmehr bestimmt die Produktionseffizienz eines Individuums den ökonomischen Wert der Ausbildung. Während sich die Schulbildung statistisch messen lässt, ist die Berufserfahrung, die einen wesentlichen Anteil an der Produktivität besitzt, nur begrenzt messbar. Indikatoren hierfür sind die Erwerbszugehörigkeitsdauer sowie die Art der ausgeübten Beschäftigung; Vergleiche der Fähigkeiten verschiedener Individuen sind jedoch nicht ohne weiteres möglich. Unterteilt man Ausbildung und Berufserfahrung in formelle bzw. informelle Ausbildung, so kann die formelle Ausbildung anhand der investierten Zeit gemessen werden, während die informelle Ausbildung eine Funktion des Alters ist (vgl. Mincer (1958), S. 287).

Problematisch ist auch die Messbarkeit der Investitionen in die Bildung in Form von finanziellem Aufwand. Bildung ist nur dann mit direkten Kosten verbunden, wenn Schulgeld oder Studiengebühren zu zahlen sind. Die Opportunitätskosten der Ausbildung, die umso höher sind, je mehr bereits in Bildung investiert wurde, sind jedoch nicht eindeutig zu quantifizieren. Auch die Förderung der Ausbildung von Kindern durch die Familie lässt sich nicht in Geldeinheiten ausdrücken. Zuletzt besteht darüber hinaus die Problematik, dass ein Studium zwar auch die individuelle Produktivität erhöht, indem beispielsweise analytische Fähigkeiten geschult werden. Eine exakte Trennung zwischen den investiven und den konsumtiven Aspekten der Ausbildung ist jedoch nicht immer möglich, denn auf der einen Seite steht das Bestreben, in der Zukunft ein entsprechend hohes Einkommen zu erzielen, auf der anderen Seite kann Bildung per se Nutzen stiftend sein. Das Studium der Philosophie kann hier als Beispiel genannt werden (vgl. Mankiw et al. (1992), S. 418f.). ${ }^{11}$

\subsubsection{Investitionen in das Bildungskapital}

Aufgrund der Tatsache, dass die Akkumulation von Bildungskapital mit Kosten und Erträgen verbunden ist, entspricht die Entscheidung über die eigene Ausbil-

${ }^{10}$ An dieser Stelle sei darauf hingewiesen, dass die Dauer der Schulbildung deren Qualität nicht uneingeschränkt wiedergibt, da sich sowohl das Wiederholen einer Klasse als auch das Phänomen eines uberdurchschnittlich langen Studiums verzerrend auswirken. Folglich bestehen Unterschiede zwischen der Ausbildungsdauer und der Effizienz, mit der Zeit in Bildung umgewandelt wird.

11 Analog zur Bildung wird im Hinblick auf die Gesundheit ebenfalls hăufig zwischen konsumtiven und investiven Zielen unterschieden (vgl. z. B. Grossman (1970)), wobei auch hier eine eindeutige Trennung nicht immer möglich ist (siehe hierzu Kapitel 4). 
dung einer ökonomischen Entscheidung, die als Investition zu verstehen ist. Investitionen in Bildungskapital sind aus ökonomischer Sicht Investitionen in das Lebenszeiterwerbseinkommen. Zur Akkumulation von Bildungskapital wird hauptsächlich Zeit benötigt. Jedes zusätzliche Ausbildungsjahr verschiebt das nach der Ausbildung erzielbare Erwerbseinkommen um ein weiteres Jahr nach hinten, so dass die Erwerbsdauer immer weiter abnimmt. Damit lassen sich die Kosten einer Ausbildung in zwei Komponenten aufspalten. Zunächst existieren direkte Kosten in Form von Studiengebühren sowie Ausgaben für Lernmittel aller Art. Den wesentlich größeren Teil der Kosten machen jedoch die indirekten Kosten in Form entgangener Lohneinkommen aus (vgl. Mincer (1958), S. 284). Die Entscheidung über ein Studium richtet sich damit nicht nur nach dem zukünftigen Einkommen, sondern auch nach dem Lebenseinkommen, das der Student verdient hätte, hätte er statt des Studiums eine praktische Ausbildung abgeschlossen. Einer durch lange Ausbildungszeiten bedingten kurzen Erwerbsphase muss folglich ein entsprechender Einkommensanstieg gegenüberstehen, damit sich derartige Investitionen lohnen. ${ }^{12}$

Analog zu physischem Kapital bestimmen sich Gewinne oder Verluste aus der Ausbildung, indem die zukünftigen Erträge diskontiert und dem Barwert der Ausbildungskosten gegenübergestellt werden. ${ }^{13}$ Eine alternative Form der Rentabilitätsanalyse erfolgt mit Hilfe der internen Rendite. Diese entspricht dem Zinssatz, bei dem der Gewinn der Investition gerade Null beträgt. Liegt die interne Rendite über dem Zinssatz alternativer Investitionen, beispielsweise von Geldanlagen auf dem Kapitalmarkt, ist die Ausbildung weiterhin die beste Alternative.

Zusätzlich zu den mit den Investitionen verbundenen Kosten mindern Abschreibungen auf das Bildungskapital die Erträge. Wird bereits Gelerntes im Zeitablauf vergessen oder aufgrund der technischen Entwicklung obsolet, muss erneut in die Bildung investiert werden, um den Bildungskapitalbestand zumindest konstant zu halten. Es handelt sich hierbei also nicht um Investitionen, die aus-

\footnotetext{
${ }^{12}$ Schultz (1961) fuhrt an, dass die Kosten der Ausbildung in der ersten Hälfte des 20. Jahrhunderts wesentlich schneller wuchsen als die Investitionen in physisches Kapital. Diese Entwicklung legt den Schluss nahe, dass die Bildung erstens nicht als reines Konsum-, sondern als Investitionsgut zu betrachten ist, und dass zweitens Investitionen in die Bildung mit hohen Erträgen verbunden sind, welche die Erträge des reinen physischen Kapitals übersteigen. Unter der Annahme, dass Bildung nicht nur die Produktivität auf dem Arbeitsmarkt erhöht, sondern sich darüber hinaus auch positiv auf die Qualităt des Konsums auswirkt, ist ein Teil der im Rahmen der Investitionen anfallenden Kosten direkt dem Konsum zuzurechnen. Dementsprechend würde sich auch die Ertragsrate der Investitionen erhöhen (vgl. Schultz (1961), S. 12f.).

${ }^{13} \mathrm{Vgl}$. hierzu insbesondere Mincer (1984).
} 
schließlich zu Beginn des Erwerbslebens getätigt werden, sondern um einen Prozess, der die gesamte Lebensdauer über anhält (vgl. Mincer (1984), S. 196). Wird aufgrund fehlender Rentabilität keine weitere oder zu wenig Zeit in die Bildung investiert, führt dieses Verhalten zu einem Absinken des Bildungskapitalstocks. Aufgrund der immer kürzer werdenden Erwerbsperiode ist die Abschreibungsrate auf das Bildungskapital umso größer, je mehr Zeit bereits in die Ausbildung investiert wurde. Insofern sind dauerhafte Weiterbildungsmaßnahmen insbesondere bei sehr gut ausgebildeten Individuen notwendig, während Personen mit geringer Bildung im Verlauf der Erwerbstätigkeit auch weniger Zeit in die Weiterbildung investieren. ${ }^{14}$

\subsubsection{Externe Effekte}

Problematisch im Hinblick auf die Investitionen in Bildungskapital sind externe Effekte. Neue Technologien und damit neues Wissen können nicht vollständig durch Patente vor Nachahmung geschützt werden. Sobald neue Produkte auf dem Markt erhältlich sind, wird dieses Wissen anderen Unternehmen zugänglich gemacht, so dass eine vollständige Geheimhaltung nicht möglich ist (vgl. Romer (1986), S. 1003). Einerseits kann dieser Wissenstransfer zwar zu neuen, verbesserten Forschungsergebnissen führen, andererseits wird das innovative Unternehmen jedoch nicht ausreichend finanziell kompensiert. Damit führen positive externe Effekte in der Forschung zu Unterinvestitionen. Gleiches gilt für die direkte Akkumulation von Bildungskapital. Können private Erträge der Bildung aufgrund einer Produktivitätssteigerung direkt über den Lohn abgegolten werden, finden soziale Erträge durch den Einzelnen keine Berücksichtigung, da der persönliche Beitrag zum durchschnittlichen Bildungskapitalbestand einer gesamten Gesellschaft in der Regel vernachlässigbar ist. Würden derartige Erträge, die erst im Aggregat erkennbar sind, in die Bildungsentscheidung integriert, würde dies zu erhöhten Bildungsinvestitionen führen.

\footnotetext{
${ }^{14}$ De facto kann sich die Höhe der Abschreibungen zwischen einzelnen Individuen unterscheiden, da die genetischen Voraussetzungen unterschiedlich sind. Darüber hinaus hängt die Halbwertszeit des erworbenen Wissens stark von der eigenen Branchenzugehörigkeit ab. So ist Wissen über die Herstellung und Verwendung von Informationstechnologien bereits wesentlich fruher veraltet als erlernte Fähigkeiten in handwerklichen Berufen oder auf dem Fachgebiet der Bürokommunikation.

${ }^{15} \mathrm{Zu}$ einem Überblick über externe Effekte in der neueren wachstumstheoretischen Literatur siehe Bodenhöfer und Riedel (1998). Eine ausfuhrliche Behandlung externer Effekte folgt in Kapitel 2.2
} 


\subsection{Gesundheit als Humankapital}

\subsubsection{Definitionsansätze}

In der einschlägigen Literatur sind nur wenige Definitionsansätze für den $\mathrm{Be}$ griff der Gesundheit zu finden. Aufgrund der fehlenden Messbarkeit von Gesundheit als Ganzes wird oftmals ausschließlich von einer guten Gesundheit als Ziel gesprochen, ohne dieses Ziel jedoch näher zu spezifizieren. Eine sehr eng gefasste Definition der Gesundheit findet sich in der Satzung der World Health Organization (WHO), in der es heißt: „Health is a state of complete physical, mental and social well-being and not merely the absence of disease or infirmity" (Sachverständigenrat für die Konzertierte Aktion im Gesundheitswesen (1994), Ziffer 4). Die Gesundheitsdefinition der WHO bezieht sich damit nicht nur auf den biologischen Gesundheitsbegriff, sondern berücksichtigt darüber hinaus auch andere Dimensionen der Gesundheit im Sinne physischen, psychischen und sozialen Wohlbefindens.

Die WHO-Definition ist insofern sehr restriktiv, als dass hiernach nur derjenige gesund ist, dessen Wohlbefinden vollkommen ist. Diese Auffassung der Gesundheit impliziert daher, dass bei einer wörtlichen Anwendung kaum jemand gesund sein kann, da beispielsweise physische Beeinträchtigungen eher die Regel denn die Ausnahme sind. Zudem kann sich eine Person gesund fühlen, obwohl eine objektive Beeinträchtigung vorliegt. So wird sich jemand, der eine geeignete Sehhilfe benötigt, ansonsten jedoch schmerzfrei ist, keineswegs als ungesund oder sogar krank bezeichnen. Daher ist an dieser Stelle zu konstatieren, dass Gesundheit weniger als ein objektiv messbarer als vielmehr als ein subjektiv empfundener Zustand $\mathrm{zu}$ verstehen ist (vgl. Zweifel und ZyssetPedroni (1992), S. 41).

Etwas allgemeiner ist die Definition des Sachverständigenrates für die Konzertierte Aktion im Gesundheitswesen. In dieser Definition wird nicht mehr der $\mathrm{Zu}-$ stand der Gesundheit per se, sondern vielmehr die Zielsetzung in den Vordergrund gestellt. So heißt es: „[D]er Begriff von Gesundheit zielt auf die Freiheit des Menschen von der Bedrohung der Gesundheit durch Krankheit, der Bedrohung der Funktionalität (Lebensqualität/Produktivität) und Leid (z. B. Schmerz, Depression)" (Sachverständigenrat für die Konzertierte Aktion im Gesundheitswesen (1994), Ziffer 1). Der Begriff „Lebensqualität“ beschreibt in diesem Zusammenhang die passive Möglichkeit der Lebensgestaltung, während der $\mathrm{Be}$ griff „Produktivität“ einen aktiven Anteil an der Gestaltung impliziert. Indem lediglich Zielvorgaben gesetzt werden, ist es laut dieser Definition möglich, sich stets gesund zu fühlen, obwohl sich die Produktivität wie auch die Lebensqualität mit zunehmendem Alter verändern (vgl. Sachverständigenrat für die Konzertierte Aktion im Gesundheitswesen (1994), Ziffer 1). 
Im Rahmen einer Allgemeindefinition lässt sich der Gesundheitsbegriff in zwei Komponenten aufspalten. Erstens existiert ein physiologischer Gesundheitsbegriff, indem Gesundheit als Absenz von Krankheit interpretiert werden kann (vgl. Leu et al. (1986), S. 158). Zahlreiche Indikatoren der Gesundheit sind objektiv messbar und geben somit Aufschluss über die rein körperliche Leistungsfähigkeit eines Individuums. Dieser objektive Gesundheitsbegriff impliziert allerdings, dass Personen mit identischen körperlichen Voraussetzungen auch hinsichtlich ihrer physischen Belastbarkeit identisch sind.

Diese Annahme erscheint aufgrund der offensichtlichen Unterschiede zwischen einzelnen Individuen nahezu gleicher körperlicher Verfassung zu vereinfachend. Insofern ist eine Erweiterung um eine subjektive Komponente, die das persönliche Befinden widerspiegelt, notwendig und sinnvoll. Für die ökonomische Bewertung der Arbeitskraft spielt die eigene Einschätzung des Gesundheitszustandes insofern eine wesentliche Rolle, als dass hierüber nicht nur die Leistungsbereitschaft beeinflusst wird, sondern auch die Inanspruchnahme von Fehlzeiten, da Arbeitsunfähigkeit infolge von Krankheit zumeist eine subjektive Entscheidung darstellt.

Dieser zuletzt genannte Zusammenhang beschreibt eine von zwei wichtigen Eigenschaften, die eine Einordnung der Gesundheit in den ökonomischen Kontext ermöglichen (vgl. Breyer et al. (2005), S. 5): Zum einen kann nur derjenige, der gesund ist, auf dem Arbeitsmarkt Einkommen erzielen, und zum Zweiten wird der Nutzen, der aus diesem Einkommen gezogen werden kann, durch den Gesundheitszustand bestimmt. Hier zeigt sich wiederum die Dehnbarkeit des Gesundheitsbegriffs. Nicht nur die grundsätzliche Erzielung von Erwerbseinkommen ist von der Gesundheit abhängig, sondern es existieren darüber hinaus $\mathrm{Ab}$ stufungen in der Entlohnung aufgrund von gesundheitsbedingten Produktivitätsunterschieden von Erwerbstätigen. So ist die individuelle Gesundheit zwar eine notwendige Bedingung für Erwerbstätigkeit, darüber hinaus beeinflusst sie jedoch auch die Höhe des zu erwartenden Erwerbseinkommens.

Das als Zweites angeführte Nutzenargument unterstützt die Definition eines subjektiven Gesundheitsbegriffs. So erscheint es sinnvoll, dass sich die Wertschätzung eines bestimmten Konsumgutes, bspw. eines beliebigen Nahrungsmittels, mit dem Gesundheitsbefinden verändert. In Zweifel und Zysset-Pedroni (1992) findet sich zudem noch die soziale Gesundheit als drittes Merkmal, über die soziale Kontakte und Beziehungspersonen in den allgemeinen Gesundheitszustand mit einfließen. Eine detaillierte Aufstellung dieser drei Gesundheitsdimensionen sowie deren Teilkomponenten ist in Tabelle 1 dargestellt. 
Tabelle 1: Die wichtigsten Gesundheitsdimensionen in der Health Insurance Study der Rand Corporation

\begin{tabular}{|c|c|}
\hline undheit & II Psychische Gesundheit \\
\hline $\begin{array}{l}\text { - Selbstpflege (Essen, Körperpflege) } \\
\text { - Mobilität (Bewegungsbereich) } \\
\text { - Phys. Aktivität (Gehen, Laufen) } \\
\text { - Soziale Rolle (Arbeit) } \\
\text { - Haushaltsaktivitäten } \\
\text { - Freizeitaktivitäten }\end{array}$ & $\begin{array}{l}\text { (Nur allgemein akzeptierte, häufige, } \\
\text { beeinflussbare Störungen) } \\
\text { - Depression } \\
\text { - Angstzustände } \\
\text { - Emotionale Kontrolle } \\
\text { - Selbstwertgefühl }\end{array}$ \\
\hline III Soziale Gesundheit & $\begin{array}{c}\text { IV Generelle } \\
\text { Gesundheitseinschätzung }\end{array}$ \\
\hline $\begin{array}{l}\text { - Beziehungspersonen } \\
\text { - Kontakte (Besuche, besucht wer- } \\
\text { den, Telefonate, Briefe) } \\
\text { - Mitwirken in Gemeinschaften (Kir- } \\
\text { che, Club, Team) }\end{array}$ & $\begin{array}{l}\text { - Resistenz und Empfindlichkeit } \\
\text { - Besorgnis } \\
\text { - Krankheitsbewusstsein } \\
\text { - Allgemeine Einschätzung der eige- } \\
\text { nen Gesundheit }\end{array}$ \\
\hline
\end{tabular}

Quelle: Nach Brook et al. (1979), zitiert in Leu et al. (1986), S. 46.

Wird ein rein physiologisches Konzept der Gesundheit verfolgt, lassen sich zahlreiche Eigenschaften des individuellen Gesundheitszustandes nicht hinreichend abbilden. Insofern ist es notwendig, auch die Einstellung zur Gesundheit sowie die Bedeutung des Gesundheitssystems für die Inanspruchnahme medizinischer Leistungen in eine Abgrenzung der Begriffe Gesundheit und Krankheit einzubeziehen.

Eine Feststellung des allgemeinen Gesundheitszustandes nach diesen Indikatoren ist auf zwei Arten möglich. Einen Gesundheitsindex erhält man, indem man die Summe aus den drei Komponenten physische, psychische und soziale Gesundheit bildet und somit eine Zielgröße definiert. Je nach erreichtem Indexwert lässt sich so eine Person als gesund oder krank einstufen. Problematisch bei dieser rein additiven Vorgehensweise ist jedoch die Bewertung einzelner Komponenten mit Punkten, obwohl sich die individuelle Gewichtung durchaus unterscheiden kann. Eine zweite Möglichkeit besteht daher in dem Erstellen eines Gesundheitsprofils, bei dem nur einzelne Indikatoren zusammengefasst werden, so dass die Gesundheit weiter durch einzelne Komponenten definiert wird. Bei 
dieser Vorgehensweise kann das Problem der Bewertung zumindest teilweise vermieden werden (vgl. Leu et al. (1986)).

\subsubsection{Messbarkeit}

Ein besonderes Handicap bei der statistischen Verwendung von Gesundheit besteht darin, „dass Gesundheit eine mehrdimensionale qualitative Variable ist, die nicht direkt beobachtet oder gemessen werden kann" (Leu und Doppmann (1986), S. 162). Stattdessen müssen Indikatoren herangezogen werden, die den individuellen Gesundheitszustand so exakt wie möglich wiedergeben. Problematisch ist in diesem Zusammenhang, dass stets das Risiko einer Missspezifikation des Indikators besteht. Gerade aufgrund der Uneinheitlichkeit des Gesundheitsverständnisses ist entsprechend zu erwarten, dass auch die Indikatoren den tatsächlichen Gesundheitszustand nicht eins zu eins wiedergeben.

Ein zweites Problem besteht in Messfehlern. Diese können im Hinblick auf objektiv messbare Gesundheitsdaten wie Blutwerte oder Puls zwar nahezu vermieden werden, gerade in Bezug auf das Gesundheitsempfinden ist eine Vermeidung jedoch nicht möglich. Die subjektive Einschätzung des Gesundheitszustandes lässt sich nur über Befragungen feststellen, die in der Regel Interpretationsspielräume offen lassen und so zu Verzerrungen führen. Wird beispielsweise gefordert, die eigene Gesundheit in fünf Kategorien von sehr gut bis sehr schlecht einzuordnen, so ist davon auszugehen, dass unterschiedliche soziale Gruppen die Schwellenwerte von einer zur nächsten Kategorie auch unterschiedlich setzen (vgl. Hernández-Quevedo et al. (2004), S. 5).

Traditionelle Indikatoren für die statistische Messung von Gesundheit sind Maße wie die Kindersterblichkeit, Mortalitätsraten sowie Todesursachen (siehe hierzu Zweifel und Zysset-Pedroni (1992), S. 47f.). Ein wesentlicher Vorteil dieser Maße ist die Verfügbarkeit statistischer Daten, wobei sich dieser Vorteil aufgrund der problematischen Datenerhebung maßgeblich auf Untersuchungen in Entwicklungsländern bezieht. Nachteilig bei diesen Konzepten ist insbesondere, dass nichttödliche Krankheiten nicht berücksichtigt werden, obwohl hauptsächlich chronische Krankheiten ohne direkten Einfluss auf die Mortalität in den Industrieländern dominieren. Darüber hinaus werden über diese Indikatoren subjektive Einschätzungen nicht abgebildet, so dass es sich bei der Sterblichkeit um einen relativ schwachen Indikator handelt, der einer Selbsteinschätzung des Gesundheitszustandes nicht vorzuziehen ist. ${ }^{16,17}$

\footnotetext{
${ }^{16}$ Aus diesen Gründen wird im Rahmen der empirischen Untersuchung (Kapitel 8) die Selbsteinschätzung als abhängige Variable verwendet.
} 


\subsubsection{Gesundheit als Investitionsgut}

Insbesondere seit den Arbeiten von Mushkin (1962) und Grossman (1972) existiert das Konzept von der Gesundheit als Investitionsgut. Physische Anlagegüter kennzeichnen sich unter anderem dadurch, dass ihr ökonomischer Wert durch Investitionen gesteigert oder durch übermäßigen Gebrauch gemindert werden kann. Gleiches gilt für die Gesundheit. Ausgehend von einem Status Quo unterliegt die individuelle Gesundheit stets einem Wandel in Form von Abschreibungen durch den natürlichen Alterungsprozess sowie durch gesundheitsschädigendes Verhalten (beispielsweise Stress oder Zigaretten- und Alkoholkonsum). Um einen bestimmten Gesundheitszustand zu erhalten, müssen folglich das übrige Verhalten angepasst und eventuell medizinische Leistungen nachgefragt werden. Die sich hieraus ergebenden positiven Veränderungen der Gesundheit können als Erträge von Investitionen interpretiert werden.

Ebenfalls analog zu übrigen Investitionsgütern ist Gesundheit sowohl als Bestands- als auch als Flussgröße zu betrachten (vgl. Williams (1985), S. 1024f.). Gesundheit selbst stiftet als Bestandsgröße direkten Nutzen. Darüber hinaus bestimmt sie als Flussgröße die gesund verbrachte Zeit, die wiederum für sämtliche Aktivitäten notwendig ist. Im Hinblick auf die Erwerbstätigkeit bestimmt die Gesundheit das effektive Arbeitsangebot, also die Arbeitszeit sowie die durchschnittliche Produktivität und damit auch das Erwerbseinkommen. Je besser die Gesundheit subjektiv ist, desto mehr Zeit wird gesund verbracht und steht somit für Erwerbszwecke zur Verfügung. Zudem wird gesunde Zeit für den Konsum sowie für die Haushaltsproduktion verwendet, deren Ergebnis nicht einfach messbar ist. Die Nutzenstiftung eines Konsumgutes ist folglich von der Gesundheit abhängig, indem nicht jedes Konsumgut bei unterschiedlichen Gesundheitszuständen gleichermaßen umfassend genutzt werden kann.

Unter der Annahme, dass eine gute Gesundheit die ökonomische Handlungsfähigkeit gegenüber eines schlechten Gesundheitszustandes insofern beeinflusst, als dass die Herstellung von anderen Gütern und Leistungen mit einer erhöhten Produktivität erfolgt, kann man daher von der Gesundheit als einem Kapitalgut sprechen (vgl. Breyer et al. (2005), S. 74). Die Akkumulation von Gesundheitskapital erfolgt hierbei über gezielte Investitionen in die Gesundheit. Die folgende Abbildung 2 zeigt Einflussfaktoren des individuellen Gesundheitszustandes auf, die zumindest teilweise durch das Verhalten beeinflussbar sind.

${ }^{17} \mathrm{Zu}$ den unterschiedlichen ökonomischen Bewertungsansätzen, z. B. QALY (Quality Adjusted Life Year) oder HYE (Healthy-years Equivalent), siehe beispielsweise Ried (1998a) oder Dolan (2000). 
Abbildung 2: Bestimmungsfaktoren des Gesundheitszustandes

\begin{tabular}{|c|c|c|}
\hline \multicolumn{3}{|c|}{ Bestimmungsfaktoren des Gesundheitszustandes } \\
\hline $\begin{array}{l}\text { Demographische } \\
\text { GröBen: } \\
\text { Alter } \\
\text { Geschlecht } \\
\text { Familienstand } \\
\text { Familiengröße } \\
\text { usw. }\end{array}$ & Gesundheitszustand & $\begin{array}{l}\text { Soxioøkonomische } \\
\text { Groben: } \\
\text { Einkommen und Vermögen } \\
\text { Beruf } \\
\text { Ausbildung } \\
\text { Wohnort } \\
\text { Stellung im Arbeitsprozess } \\
\text { usw. }\end{array}$ \\
\hline $\begin{array}{l}\text { Individuelle GroBen: } \\
\text { Erbfaktoren } \\
\text { Krankheitsgeschichte } \\
\text { Lebensgewohnheiten } \\
\text { (Gesundheits- und } \\
\text { Risikoverhalten) } \\
\text { Ernährungsverhalten } \\
\text { Gesundheitsbewusstsein } \\
\text { Symptomaufmerksamkeit } \\
\text { usw. }\end{array}$ & $\begin{array}{l}\text { Strukturelle } \\
\text { Groben: } \\
\text { Arbeitsbedingungen } \\
\text { Wohnverhältnisse } \\
\text { Verkehrssicherheit } \\
\text { Versicherungsschutz } \\
\text { Lohnfortzahlung } \\
\text { Umweltqualität }\end{array}$ & $\begin{array}{l}\text { Medirainische GröBen: } \\
\text { Angebot an medizinischen } \\
\text { Leistungen } \\
\text { Medizinischer } \\
\text { Wissensstand } \\
\text { Medizinisch-technische } \\
\text { Entwicklung } \\
\text { Zugang zum medizinischen } \\
\text { System und Verfugbarkeit } \\
\text { Überweisungsverhalten } \\
\text { usw. }\end{array}$ \\
\hline
\end{tabular}

Quelle: Zdrowomyslaw und Dürig (1997), S. 2.

Insgesamt werden die einzelnen Einflussgrößen in fünf verschiedene Kategorien untergliedert. Während die individuellen Größen Aufschluss über die Bereitschaft zu Investitionen in die Gesundheit geben, bestimmen die sozioökonomischen Größen den Rahmen, in dem Investitionen möglich sind. So sichert ein hohes Einkommen nicht nur den Konsum von medizinischen Leistungen per se, sondern es ermöglicht darüber hinaus bspw. den Konsum hochwertiger Nahrungsmittel, die sich positiv auf die Gesundheit auswirken. Demgegenüber werden die medizinischen Faktoren in der Regel direkt mit der Gesundheit in Verbindung gebracht, wobei hier nicht das Individuum als Produzent seiner Gesundheit erachtet wird, sondern der behandelnde Arzt.

Diese einzelnen Größen sind insofern als interdependent anzusehen, da der Erfolg medizinischer Behandlungsmethoden immer auch von der Handlung des erkrankten Individuums abhängt. Darüber hinaus handelt es sich hierbei im Wesentlichen um kurative Maßnahmen, während die individuellen Größen vermehrt die Bereitschaft zur Prävention widerspiegeln. Weiterer Erklärungsgehalt 
wird zudem den Kategorien der demographischen sowie der strukturellen Größen zugeschrieben.

Gegen die Verwendung des Gesundheitskapitalbegriffs lassen sich folgende Argumente anbringen (vgl. hierzu Breyer et al. (2005), S. 75f.): Erstens unterliegt die Gesundheit im Verhältnis zu anderen Kapitalgütern einer mangelnden Steuerbarkeit. Es kann weder Gesundheitskapital auf Vorrat akkumuliert werden, noch besteht de facto die Möglichkeit, Lagerbestände abzubauen. Zudem existiert keine Produktionsfunktion, die sämtliche direkten Input-Output-Beziehungen zwischen den konsumierten Gesundheitsleistungen und dem Gesundheitskapitalbestand abbildet. Es existieren stets Faktoren, welche die Gesundheit unvorhergesehen beeinflussen.

Nun ist zwar keine direkte Vorratsproduktion möglich, indirekt erfolgt diese jedoch über eine Senkung der Erkrankungswahrscheinlichkeit bei entsprechend „gesunder" Lebensweise. Somit können Investitionen in die Gesundheit Erkrankungen zwar nicht immer vermeiden, den allgemeinen Gesundheitszustand jedoch verbessern und so die Wahrscheinlichkeit einer Krankheit mindern.

Als zweites Argument gegen die Interpretation von Gesundheit als Kapitalart wird die mangelnde Handelbarkeit angeführt. Während das Ergebnis originärer Produktionsprozesse stets zum Verkauf angeboten wird, ist dies bei beiden Humankapitalarten, d. h. Gesundheit und Bildung, nicht möglich. Breyer et al. (2005) entkräften dieses Argument insofern, als dass jedes Individuum einen Handel mit sich selbst eingeht, indem „es zur Erreichung eines verbesserten Gesundheitszustandes auf andere Dinge verzichten muss“ (S. 76). Analog zu übrigen Produktionsprozessen werden Inputgüter benötigt, die je nach Krankenversicherungssystem auf dem Markt für Gesundheitsgüter bezogen werden können. Eine Handelbarkeit mit dem Gut Gesundheit ergibt sich zudem insofern, als dass dieses zwar nicht einzeln am Markt angeboten werden kann, jedoch in Verbindung mit dem übrigen Humankapital als Arbeitskraft zur Verfügung steht. Der Preis des "Gutes“ Gesundheit spiegelt sich in einem Teil des Arbeitslohnes wider.

Auch wenn die vorangegangenen Argumente gegen die Behandlung der Gesundheit als Investitionsgut relativiert werden können, existieren zwei wesentliche Unterschiede zu Kapitalgütern im betriebswirtschaftlichen Sinne: Die Auswirkungen gesundheitsfördernden oder auch schädigenden Verhaltens sind zumeist langfristiger Natur, so dass die Erträge aus den Investitionen nicht eindeutig beobachtbar oder bestimmten Handlungen zurechenbar sind. Vielmehr handelt es sich bei der Gesundheit um ein zu komplexes „Gut“. Der zweite Unterschied besteht in der mangelnden Wiederbeschaffbarkeit. Teilweise sind Ge- 
sundheitsschäden irreparabel, der ursprüngliche Gesundheitszustand ist nicht reproduzierbar. Dieses Argument gegen die Behandlung von Gesundheit als Kapitalstock wird jedoch mit zunehmendem technischem Fortschritt im Gesundheitswesen immer weiter abgeschwächt, so dass nachfolgend weiterhin von der Gesundheit als Investitionsgut ausgegangen wird.

\subsubsection{Externe Effekte im Gesundheitswesen}

Wie sich anhand der unterschiedlichen Definitionsansätze zeigt, können Investitionen in die Gesundheit aus unterschiedlichen Motivationen heraus getätigt werden. Wird einer guten Gesundheit direkte Nutzenstiftung zugeschrieben, sollte es ein primäres Ziel sein, unabhängig von anderen Individuen in die eigene Gesundheit zu investieren und diese auf diese Weise stetig zu erhalten oder zu verbessern. Wird die Gesundheit hingegen als ökonomischer Produktionsfaktor gesehen, der mit anderen Humankapitalarten sowie physischem Kapital in einer Konkurrenzbeziehung stehen kann, so unterliegt auch die Entscheidung über die optimale Investitionshöhe ökonomischem Kalkül. Investitionen in die Gesundheit werden unterlassen, wenn Investitionen in das Bildungskapital höhere Produktivitätszuwächse versprechen und damit zu höherem Erwerbseinkommen führen. Die Nutzenmaximierung erfolgt in diesem Fall über das Einkommen und damit nur indirekt über die Gesundheit. In diesem Zusammenhang ist der Einfluss externer Effekte auf die Investitionen in die Gesundheit zu sehen, da hierüber die Investitionsentscheidung nachhaltig beeinflusst wird.

Externe Effekte liegen vor, wenn die Handlungen eines Individuums den Nutzen eines anderen Individuums beeinflussen, das individuelle Nutzenmaximum aufgrund nicht kalkulierbarer Einwirkungen von außen also nicht erreicht werden kann. Folglich ist der Konsum medizinischer Leistungen durch Individuum 1 als ein positiver externer Effekt $\mathrm{zu}$ verstehen, wenn sich hieraus positive Nutzenwirkungen bei Individuum 2 ergeben (vgl. Breyer et al. (2005), S. 175). Negative Externalitäten hingegen liegen dann vor, wenn das zweite Individuum entsprechend Nutzeneinbußen erfährt. Beispiele für negative externe Effekte sind sämtliche ansteckenden Krankheiten, da diese nicht nur die verfügbare gesund verbrachte Zeit eines Einzelnen beeinflussen, sondern darüber hinaus andere Personen durch die Krankheit betroffen sein können, so dass gesamtwirtschaftlich weniger ,gesunde“ Zeit zur Verfügung steht. Somit wirkt der externe Effekt direkt auf den Gesundheitszustand anderer Individuen ein. Zudem wird nicht nur der aktuelle Gesundheitszustand negativ beeinflusst, vielmehr führen negative externe Effekte zu vermehrten Investitionen in das Gesundheitskapital, um den ursprünglichen Gesundheitszustand wiederherzustellen. Unter bestimmten Voraussetzungen können selbst nicht ansteckende Krankheiten sowie Verletzungen als negative externe Effekte interpretiert werden, solange diese zu Arbeitsunfähigkeit führen. Liegt Vollbeschäftigung vor, können keine weiteren Arbeits- 
kräfte mehr eingestellt werden, so dass konstante Produktionsmengen nur dann erreicht werden können, wenn die Arbeitszeit ausgedehnt wird. Ebenso können institutionelle Rahmenbedingungen dazu führen, dass der Unternehmenssektor keine weiteren Arbeitskräfte nachfragt, sondern lediglich den Arbeitsumfang der bisher beschäftigten Individuen ausweitet, selbst wenn keine Vollbeschäftigung gegeben ist. Führt in diesem Zusammenhang schlechte Gesundheit nicht nur zu einer Minderung der Produktivität, sondern darüber hinaus zu einer zeitweiligen Verringerung des Arbeitsangebots (vgl. Strauss und Thomas (1998), S. 812), so muss die fehlende Arbeitszeit durch die übrigen Individuen kompensiert werden.

Neben den angeführten Auswirkungen auf den durchschnittlichen Gesundheitszustand aller Erwerbstätigen führen positive Externalitäten zu einem Minderkonsum insbesondere von präventiven Gesundheitsgütern, da nicht nur der Einzelne Vorteile aus der Gesundheitsvorsorge zieht, sondern da die Allgemeinheit durch eine generelle Senkung des Krankheitsrisikos positiv betroffen ist. In diesem Sinne verfügen Externalitäten über den Charakter eines öffentlichen Gutes, da zum einen der Konsum nicht rival ist, d.h. die Vorteile der Allgemeinheit mindern nicht den Nutzen des Einzelnen, zum anderen ist kein Ausschluss anderer von den positiven Effekten möglich. Somit besteht kein Anreiz für das einzelne Individuum, sich an der Bereitstellung von Gesundheitsleistungen, welche ihrerseits positive Externalitäten fördern, zu beteiligen (vgl. Breyer et al. (2005), S. 176).

Für die Gesundheitsversorgung ergeben sich aus diesen Überlegungen folgende Implikationen: Zum einen ist es notwendig, negative externe Effekte über ein umfassendes Angebot an medizinischen Leistungen einzudämmen, um die Auswirkungen auf den gleichgewichtigen Gesundheitszustand möglichst gering $\mathrm{zu}$ halten. Zum anderen sind präventive Maßnahmen zu fördern, da diese zwar nicht als Wachstumsmotor fungieren, jedoch das Wachstum positiv unterstützen (vgl. hierzu Sachverständigenrat für die Konzertierte Aktion im Gesundheitswesen (1996), Ziffer 271-278). Durch im Vorfeld durchgeführte gesundheitspolitische Maßnahmen können Krankheiten und damit Schäden in der ökonomischen Ressource Humankapital vermieden werden.

\subsection{Unterschiede zwischen den Humankapitalarten}

Zusammenfassend lassen sich insgesamt vier zentrale Unterschiede zwischen Bildung und Gesundheit als Humankapitalarten feststellen:

1. Ein wesentlicher Unterschied zwischen den Humankapitalarten besteht in Zeit und Dauer der Investitionen. Investitionen in die Bildung sind nicht dauerhaft, vielmehr verringern sie sich aus folgenden Gründen mit zunehmendem Alter 
(vgl. hierzu Mincer (1984), S. 198): Erstens sinken die Erträge aus den Investitionen, da mit zunehmender Lebensdauer die Erwerbsperiode immer kürzer wird. Dementsprechend verkürzt sich auch die Ertragsperiode, so dass die Erträge, in diesem Fall Lohnzuwächse, immer höher ausfallen müssen, damit sich Investitionen in die Bildung noch lohnen.

Zum Zweiten steigen die Opportunitätskosten einer Ausbildung außerhalb eines Unternehmens mit zunehmender Berufstätigkeit immer weiter an, da sich zwar die Einstiegslöhne an der direkten Ausbildung orientieren, die Lohnsteigerungen jedoch von den Leistungen im Unternehmen und damit von der $\mathrm{Be}-$ rufserfahrung abhängen. Somit kommt hier der Bildungsbegriff i. w. S. zur Anwendung.

Für die Investitionen in die Gesundheit gilt hingegen, dass diese im Zeitablauf stetig zunehmen, so dass die Ausgaben für Gesundheit mit dem Lebensalter ansteigen. ${ }^{18}$ Darüber hinaus verschiebt sich auch die Zusammensetzung der Gesundheitsinvestitionen. Während sich die Investitionen in der Jugend weitgehend auf sportliche Betätigung beschränken, steigt die Abschreibungsrate auf die Gesundheit mit dem Alter an, so dass immer mehr medizinische Leistungen in Anspruch genommen werden müssen. Folglich wird mit zunehmendem Alter immer stärker in die Gesundheit investiert, während die Bildungsinvestitionen immer weiter abnehmen.

2. Das zweite Unterscheidungsmerkmal bezieht auch auf die Übertragbarkeit der Humankapitalkomponenten zwischen den einzelnen Generationen. Das maximale Bildungskapital, das innerhalb einer Periode zur Verfügung steht, ist eine Summe aller Erkenntnisse der vorangegangenen Generationen. Im Zeitablauf ist dieses Bildungsmaximum jedoch dynamisch, da durch Erfindungen neues Bildungskapital generiert wird, so dass ein neuer maximaler Bildungskapitalstock existiert. Wird neues Wissen öffentlich zugänglich gemacht, steht dieses allen Individuen einer Gesellschaft oder eines Sprachraumes zur Verfügung.

Einer solchen zeitlichen Abhängigkeit unterliegt die Gesundheit nicht. Es ergeben sich zwar Abhängigkeiten zwischen den einzelnen Generationen, indem die genetischen Voraussetzungen die Gesundheit mit beeinflussen, negative externe Effekte übertragen sich jedoch nicht auf sämtliche nachfolgenden Generationen. Die einzige Ausnahme hierzu bilden Schwangere, deren Verhalten sich direkt auf die Gesundheit der Kinder auswirkt. Im Allgemeinen ist das Gesundheitsverhalten der älteren Familienmitglieder für die individuelle

${ }^{18}$ Siehe hierzu Ulrich (2001). 
Gesundheit jedoch nur bedingt ausschlaggebend, vielmehr determiniert der technische Fortschritt, mit dessen Hilfe mehr Krankheiten geheilt werden können, die Gesundheit. Gleiches gilt für positive externe Effekte, da sich Impfungen einer Generation nicht direkt auf ihre Nachfolgegeneration übertragen.

Derartige Abhängigkeiten lassen sich auch auf die Arbeitsproduktivität übertragen. Während die Bildung die Arbeitsproduktivität positiv beeinflusst, erweitert ein hoher gesellschaftlicher Gesundheitskapitalstock die Zahl der gegenwärtig verfügbaren Arbeitskräfte, ein direkter Einfluss auf nachfolgende Generationen besteht nicht (vgl. Mushkin (1962), S. 132-135). Somit ist die Bildung ein Entwicklungsprozess über alle Generationen, während die Produktivität, die aus der Gesundheit folgt, mit dem Tod unwiederbringlich verloren geht.

3. Sowohl Investitionen in die Bildung als auch Investitionen in die Gesundheit sind mit direkten und indirekten Kosten verbunden. Die direkten Kosten umfassen jeweils den Konsum von Gütern, die für die Investition benötigt werden. Hierzu zählen Lernmittel und Studiengebühren im Rahmen der Bildung sowie sämtliche medizinischen Leistungen im Gesundheitswesen. Da diese Güter sämtlich frei am Markt gehandelt werden, besteht hier kein Unterschied in ihrer Art, so dass diese im Zusammenhang mit den Investitionen als gleich zu bewerten sind.

Auch die indirekten Kosten sind auf den ersten Blick gleich, handelt es sich doch jeweils um das entgangene Erwerbseinkommen. Wird in die Bildung investiert, muss während der Ausbildung vollständig oder teilweise auf Erwerbseinkommen verzichtet werden. Für die Gesundheit gilt hingegen, dass indirekte Kosten genau dann entstehen, wenn Investitionen unterlassen wurden. Wird eine Krankheit behandelt, dient dies dem erneuten Aufbau des Gesundheitskapitalstocks und damit dem Abbau indirekter Kosten, indem die Erwerbsfähigkeit wiederhergestellt wird. Somit verursachen Investitionen in die Bildung indirekte Kosten, während Investitionen in die Gesundheit diese reduzieren oder bestenfalls vermeiden.

4. Ein weiterer Unterschied zwischen Bildung und Gesundheit liegt in der Versicherbarkeit des Risikos. Hier ist insbesondere der Einfluss der Abschreibungen auf den jeweiligen Humankapitalstock relevant. Eine Krankenversicherung entspricht einer Versicherung gegen ein Absinken des Gesundheitskapitalstocks. Auch wenn Krankheiten zunächst ein subjektives Empfinden zugrunde liegt, sind diese vielfach objektiv nachweisbar. Dies gilt für Knochenbrüche ebenso wie für Viruserkrankungen oder Organfehlfunktionen. 
Insbesondere der technische Fortschritt im Gesundheitswesen führt zu immer neuen Diagnosemöglichkeiten und damit zu verbesserten Behandlungen von Krankheiten. Ebenso können die Kosten für präventive Maßnahmen durch die Versicherung abgedeckt sein, solange die Maßnahmen für die Versicherung beobachtbar sind.

Gegen ein Absinken des Bildungskapitals kann hingegen keine Versicherung abgeschlossen werden, da dieses nicht objektiv messbar ist. Der Begriff Abschreibungen impliziert, dass sich der bis zu einem Zeitpunkt akkumulierte Bildungskapitalstock verringert. Bereits erworbenes Wissen wird in einem stetigen Prozess vergessen und muss daher regelmäßig gefestigt oder erneut erworben werden. Um sich gegen das Risiko des Vergessens versichern zu können, müsste zu jedem beliebigen Zeitpunkt eine Sollvorstellung über den Wissensbestand existieren. Wie viel Bildungskapital bereits akkumuliert wurde, kann jedoch nach Berücksichtigung der Abschreibungen nicht mehr bestimmt werden. Es ist zwar möglich, sich gegen Arbeitslosigkeit oder sogar Erwerbsunfähigkeit zu versichern, so dass Verdienstausfälle abgesichert sind. Entgangene Lohnsteigerungen sind jedoch ausschließlich hypothetisch festzustellen und daher nicht versicherbar. Auch kann entgangene Berufserfahrung aufgrund zeitweiliger Arbeitslosigkeit nicht quantifiziert werden.

Gesundheit und Bildung sind zwei Komponenten des Humankapitals, denen der Charakter des Investitionsgutes gemein ist. Unterschiede bestehen hingegen im zeitlichen Horizont der Investitionen, in der Kostenstruktur sowie in der Versicherbarkeit. Gemäß dem Investitionskonzept sind beide Humankapitalkomponenten als Substitute zu betrachten. Darüber hinaus ist eine gute Gesundheit Voraussetzung für den Erfolg von Bildungsinvestitionen, während Bildung wiederum den Einsatz von Gesundheitsleistungen positiv determiniert. Somit besteht darüber hinaus ein komplementärer Zusammenhang. 


\section{Humankapital als Wachstumsfaktor}

\subsection{Grundüberlegungen}

Frühe wachstumstheoretische Ansätze (z. B. Solow (1956)) betrachten allein die Kapitalintensität, also die Kapitalausstattung je Arbeitsplatz, um Wirtschaftswachstum zu erklären und eine gleichgewichtige Wachstumsrate des Einkommens herzuleiten. Unterschiede in den Wachstumsraten verschiedener Länder lassen sich so im einfachsten Fall über Unterschiede im Bevölkerungswachstum begründen.

Über diesen Ansatz gehen wachstumstheoretische Modelle hinaus, die zusätzlich zum physischen Kapital auch das Humankapital berücksichtigen. So vertritt beispielsweise Schultz (1961) nur kurze Zeit nach Solow die Ansicht, dass Investitionen in physisches Kapital nur so lange nutzbar sind, wie das Humankapital für die Anwendung neuer Technologien ausreicht. Diese Annahme impliziert, dass menschliche Fähigkeiten und physisches Kapital nicht in gleichem Maße wachsen, sondern dass vielmehr das Humankapital gesamtwirtschaftlich einen limitierenden Faktor darstellt.

Allgemein lassen sich die Auswirkungen von Humankapitalinvestitionen auf das Wachstum in eine quantitative und eine qualitative Dimension unterteilen. Die Zahl der verfügbaren Arbeitskräfte sowie die Arbeitsstunden beschreiben die quantitative Komponente, indem die in einem bestimmten Zeitraum insgesamt zur Verfügung stehende Arbeitskraft gemessen wird. Hier ist insbesondere die Gesundheit entscheidend, da diese das Wachstum der erwerbstätigen Bevölkerung sowie die zur Verfügung stehende Arbeitszeit determiniert. Die qualitative Komponente hingegen beschäftigt sich mit der Frage, mit welcher Qualität Arbeiten durchgeführt werden, so dass hierzu sowohl die Bildung in Form von individuellen Fähigkeiten und persönlichem Wissen als auch wiederum die Gesundheit, welche die Produktivität positiv beeinflusst, zu zählen sind. Folglich sind nicht nur Investitionen in physisches Kapital als Determinante des Wachstums zu sehen, sondern auch Investitionen in Humankapital.

Die Erträge beider Humankapitalkomponenten sind direkt mit der menschlichen Arbeitskraft verbunden. Wesentliches Ziel der Investitionen ist es daher, die Produktivität und damit den Wert menschlicher Arbeit zu steigern. Im Gegensatz zum physischen Kapital ist eine genaue Abgrenzung von Konsum von und Investitionen in Humankapital indes durch die Personenbindung kaum möglich.

Eine Einteilung privater Ausgaben unter Investitionsaspekten findet sich bei Schultz (1961). Dieser teilt sämtliche privaten Ausgaben in drei Kategorien ein: 
in reine Konsumausgaben, die keinerlei Auswirkungen auf Bildung oder Gesundheit besitzen, in reine Investitionsausgaben, die ohne direkte Nutzenstiftung sind und ausschließlich den Investitionen dienen, und in Mischausgaben, die eine Kombination aus beiden Ausgabenarten darstellen. Allerdings ist eine derart exakte Abgrenzung problematisch, wie das Beispiel des Nahrungsmittelkonsums zeigt: Solange der Konsum zusätzlicher Nahrungsmittel die physische Arbeitskraft erhöht oder die Produktivität steigert, besitzen diese Investitionsgutcharakter, da sich eine Steigerung des Humankapitals durch eine Verbesserung der individuellen Gesundheit ergibt. Werden jedoch darüber hinaus Nahrungsmittel konsumiert und stellt sich hierdurch keine weitere Verbesserung der Gesundheit ein, handelt es sich um ein reines Konsumgut. Im negativen Fall sind diese zusätzlich konsumierten Nahrungsmittel sogar der Gesundheit abträglich. Letztlich ist der Übergang von einem Investitions- hin zu einem Konsumgut jedoch subjektiv und damit fließend, so dass wohl die meisten Güter zu der dritten Kategorie zu zählen sind. Das Gleiche gilt für Investitionen in die Bildung.

\subsection{Die Bedeutung der Bildung für das Wirtschaftswachstum}

\subsubsection{Wachstumstheoretische Ansätze}

Die Diskussion über die Bedeutung der Bildung für das Wirtschaftswachstum gestaltet sich in der Literatur keineswegs einheitlich. Es existieren zahlreiche wachstumstheoretische Ansätze, die nach folgenden Gesichtspunkten unterschieden werden können:

Zunächst lassen sich die einzelnen Modelle in die Bereiche der neoklassischen sowie der endogenen Wachstumstheorie einordnen. Diese Ansätze unterscheiden sich maßgeblich in der Überlegung, ob technischer Fortschritt exogen gegeben ist oder aber mit Hilfe von Investitionen in Forschung und Entwicklung generiert werden kann. Ein zweites Unterscheidungsmerkmal besteht in der Frage, ob die Rolle des Humankapitals als Bestands- oder aber als Stromgröße zu sehen ist. Somit ist zu unterscheiden, ob das erreichte Bildungsniveau innerhalb einer Volkswirtschaft das Wachstum entscheidend beeinflusst oder ob die Bildungskapitalakkumulation per se von Interesse ist. Ein dritter Punkt, in dem sich die einzelnen wachstumstheoretischen Ansätze unterscheiden, bezieht sich auf die Annahme fallender oder zumindest konstanter Skalenerträge. Zum Vierten bestehen unterschiedliche Ansätze dahingehend, ob Bildung primär die Produktivität der Arbeitskraft beeinflusst und so zu Ergebnissteigerungen im Produktionssektor führt oder aber, ob die Bedeutung von Bildungskapital für das Wirtschaftswachstum hauptsächlich über den Forschungs- und Entwicklungssektor abgebildet wird, da hierüber technischer Fortschritt generiert wird. Hieraus lässt sich auch die Frage ableiten, ob letztlich der durchschnittliche Humankapitalbestand der Bevölkerung maßgeblich ist oder ob vielmehr die Position Einzelner in 
der internationalen Spitzenforschung eine Rolle spielt. Darüber hinaus weist Wissen gemeinhin den Charakter eines öffentlichen Gutes auf, weshalb die nachfolgenden Wachstumsmodelle zudem in der Berücksichtigung von Externalitäten unterschieden werden können. ${ }^{19}$ Diese Unterschiede in den zentralen Modellannahmen der wachstumstheoretischen Literatur ${ }^{20}$ sind in Abbildung 3 noch einmal überblicksartig zusammengefasst.

Abbildung 3: Bildungsbezogene Modellannahmen der Wachstumstheorie

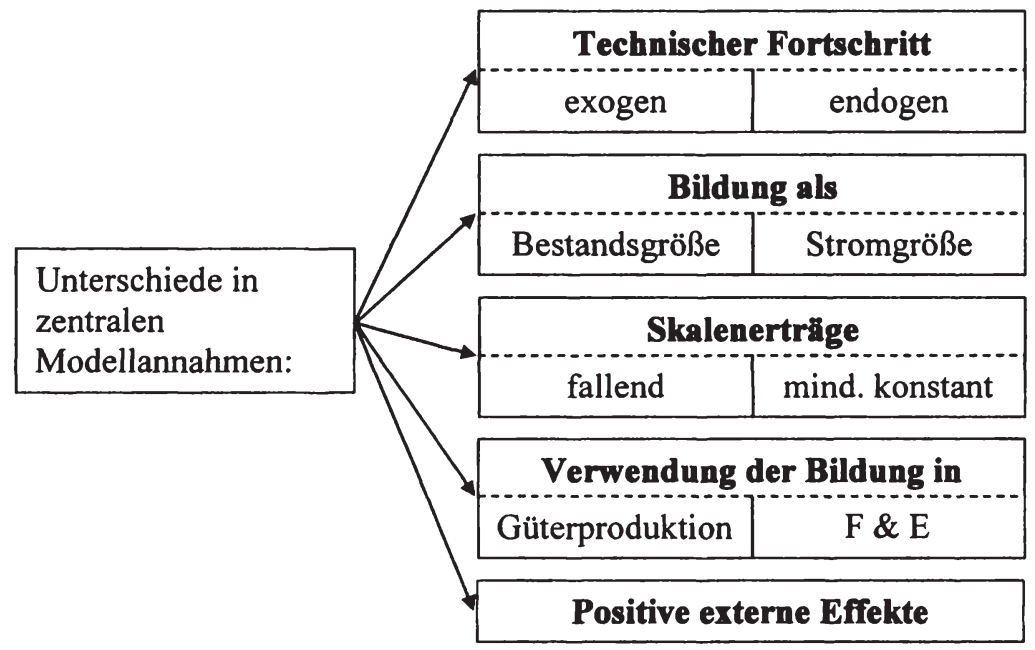

Quelle: Eigene Darstellung.

\section{Technischer Fortschritt}

Der technische Fortschritt kennzeichnet die Veränderung des theoretisch insgesamt verfügbaren Wissens im Zeitablauf. Ist dieser im Rahmen eines neoklassischen Wachstumsmodells exogen gegeben, dann ist für das Wirtschaftswachstum entscheidend, mit welcher Geschwindigkeit Innovationen in den Produktionsprozess integriert und damit langfristig standardisiert werden können. In einem solchen Zusammenhang gehen beispielsweise Nelson und Phelps (1966)

\footnotetext{
${ }^{19} \mathrm{Zu}$ einem Überblick über die Literatur der endogenen Wachstumstheorie siehe Aghion und Howitt (1999).

${ }^{20}$ Nachfolgend werden die hier genannten modelltheoretischen Grundannahmen anhand ausgewählter Literaturbeispiele erläutert und belegt. An dieser Stelle sei darauf hingewiesen, dass die Literatur lediglich einen Überblick geben soll und daher keinen Anspruch auf Vollständigkeit erhebt.
} 
davon aus, dass der zeitliche Abstand zwischen einer Erfindung und deren Integration in den Produktionsprozess durch den Bildungskapitalbestand der innovationsbereiten Unternehmer determiniert wird. ${ }^{21}$ Neue Technologien werden nicht sofort in die Produktionsprozesse implementiert, weshalb der tatsächliche Stand des technischen Fortschritts in einer Ökonomie das verfügbare Wissen nur zeitverzögert wiedergibt. Zudem erfolgt der Einfluss der Bildung auf den Modernisierungsprozess in diesem Modellansatz nur indirekt, da ein Anstieg im Bildungskapital den zeitlichen Abstand zwischen der Generierung neuen Wissens und der tatsächlichen Anwendung einer Innovation zwar verkürzen würde, das allgemeine Technologieniveau jedoch exogen gegeben und damit vom Bildungskapitalbestand unabhängig ist.

Endogener technischer Fortschritt liegt hingegen dann vor, wenn die Zahl der Innovationen durch das vorhandene Bildungsniveau beeinflusst wird und somit nicht mehr unabhängig vom Humankapitalbestand einer Volkswirtschaft ist. Mit Hilfe von Forschung und Entwicklung ist es dann möglich, neue Technologien bewusst zu entwickeln und so das maximal verfügbare Wissen stetig auszuweiten. In einem solchen Fall kann das Wissen, das tatsächlich in der Produktion eingesetzt wird, nicht mit dem theoretisch vorhandenen Know How übereinstimmen, da neues Wissen erst mit einer Zeitverzögerung in die Produktionsprozesse integriert wird. Letztlich bleibt so stets eine Differenz zwischen diesen beiden Größen dauerhaft bestehen. Diese Lücke ist umso größer, je schneller der technische Fortschritt erfolgt und umso kleiner, je mehr Bildungskapital in der Gesellschaft vorhanden ist. Dieses Ergebnis des zweiten Teils der Arbeit von Nelson und Phelps (1966) impliziert weiterhin, dass die Erträge von Humankapitalinvestitionen umso größer sind, je fortschrittlicher die Ökonomie ist, d. h. je näher sich die Volkswirtschaft an der Wissensgrenze befindet. Aufgrund dieser Annahmen der endogenen Wachstumstheorie bleiben Einkommensunterschiede zwischen den einzelnen Ländern dauerhaft bestehen.

Barro und Sala-i-Martin (1997) zeigen, dass sich die Ergebnisse der neoklassischen und der endogenen Wachstumstheorie nicht grundsätzlich ausschließen, sondern dass vielmehr auch die Einkommensposition der betrachteten Länder berücksichtigt werden muss. Langfristig hängt das Wachstum der Weltwirtschaft nach Ansicht von Barro und Sala-i-Martin von der Entwicklungstätigkeit einiger führender Nationen $a b$ und ist damit endogen, da diese das Technologieniveau aufgrund ihrer Forschungstätigkeit vorgeben. Für ärmere Länder, die sich noch nicht auf der Technologiegrenze befinden, ist der technische Fortschritt

\footnotetext{
${ }^{21}$ An dieser Stelle sei ergänzend angemerkt, dass auch übrige Rahmenbedingungen, beispielsweise gesetzliche Regelungen, die Innovationsbereitschaft bestimmen und sich so fordernd oder hemmend auf den technischen Fortschritt auswirken können.
} 
hingegen exogen, da sie gewöhnlich keine eigene Forschung betreiben. Gründe hierfür sind zum einen die mangelnde finanzielle Leistungsfähigkeit, da für Forschung in der Regel ein hoher Kapitaleinsatz erforderlich ist, zum anderen aber auch die begrenzten Fähigkeiten der Erwerbstätigen, so dass das verfügbare Bildungskapital hier ebenfalls einen restriktiven Faktor darstellt. Häufig ist Imitation von Gütern kostengünstiger als die Entwicklung einer Innovation, so dass ärmere Länder bestehende Technologien eher adaptieren, als neue zu erfinden.

\section{Bildung als Bestands- oder Stromgröße}

$\mathrm{Ob}$ es theoretisch zu Konvergenz kommen kann oder nicht, hängt zudem entscheidend von Frage ab, inwieweit einerseits das Bildungsniveau einer Gesellschaft den Wachstumsprozess unterstützt und welche Bedeutung andererseits der Bildungskapitalakkumulation zukommt. Für das Bestandskonzept spricht, dass Forschung und Entwicklung besonders humankapitalintensiv ist, weshalb die Geschwindigkeit der technologischen Entwicklung in einer Ökonomie umso größer ist, je mehr Bildungskapital bereits vorhanden ist. Ist der neueste Stand der Forschung vollständig im Produktionssektor implementiert, befindet sich eine Volkswirtschaft auf der Wissensgrenze, so dass Fortschritt und damit Wirtschaftswachstum nur aus der eigenen Forschungstätigkeit resultieren kann. Für das Bestandskonzept spricht weiterhin, dass sich technische Neuerungen bei einem hohen gesellschaftlichen Bildungsniveau schneller verbreiten können, indem neue Technologien in der Produktion relativ zeitnah zur Anwendung kommen (vgl. ebenfalls Nelson und Phelps (1966)).

Nach Ansicht von Blackburn et al. (2000) scheint es jedoch wenig sinnvoll, dass allein der Bestand, nicht aber die Akkumulation von Humankapital für das Wachstum entscheidend sein soll, denn diese Annahme wäre gleichbedeutend mit einem konstanten verfügbaren Bildungskapital. In einer Ökonomie, die nach der Technologiegrenze strebt, würde sich folglich ein Wettbewerb um hochqualifizierte Arbeitskräfte entwickeln. Die hiermit verbundenen steigenden Löhne würden die Entwicklungskosten erhöhen, die Innovationstätigkeit in der Volkswirtschaft verringerte sich. Letztlich würde hieraus Stagnation folgen, weshalb im Sinne dieser Überlegungen ein stetiger Anstieg des Bildungsniveaus für das Wirtschaftswachstum unverzichtbar ist.

Darüber hinaus ist für die Analyse zu berücksichtigen, dass sich Entwicklungsländer insbesondere dann in einem ökonomischen Aufholprozess befinden, wenn diese vermehrt in die grundlegende Ausbildung ihrer Bürger investieren und so die Verbreitung bereits existierenden Wissens beschleunigen. Je schneller dies gelingt, desto höher sind die realisierbaren Produktivitätssteigerungen in der Produktion und desto schneller können sich Länder in ihrem Einkommens- 
niveau an die Industrienationen angleichen, wodurch letztlich wieder Konvergenzprozesse ausgelöst werden.

Im Rahmen der Wachstumstheorie findet das Konzept der Stromgröße über die Humankapitalakkumulation beispielsweise bei Lucas (1988) oder Rebelo (1991) Anwendung.

\section{Skalenerträge}

Eine weitere Unterscheidungsmöglichkeit der einzelnen Wachstumsmodelle besteht in der Überlegung, ob Forschung und Entwicklung mit fallenden Skalenerträgen verbunden ist oder ob dauerhaftes Wirtschaftswachstum vielmehr mit konstanten oder sogar steigenden Skalenerträgen einhergeht. Romer (1986) geht in seinem Modellansatz beispielsweise von fallenden Skalenerträgen aus, weshalb eine Verdopplung des Forschungsaufwandes nicht zu einer Verdopplung der neuen Erkenntnisse führt (vgl. Romer (1986), S. 1003).

Rosen (1976) sowie Lucas (1988) vertreten hingegen die Ansicht, dass die Akkumulation des Bildungskapitals konstanten Grenzerträgen unterliegt, so dass diese letztlich unabhängig vom aktuellen Bestand erfolgt. Damit ist es jedem Individuum unbegrenzt möglich, in die Bildung zu investieren und Erträge zu realisieren. Bereits existierende Erkenntnisse werden zudem an die nachfolgende Generation weitergegeben, damit diese das Wissen produktiv nutzt und hieraus neues Wissen generiert.

Ein weiteres Argument für einen linearen Zusammenhang zwischen der Akkumulation von Wissen auf der einen und dem bereits implementierten Wissensbestand auf der anderen Seite und damit für die Annahme konstanter Skalenerträge findet sich bei Romer (1990), indem dieser auf Zuwächse in der Produktivität im Forschungssektor abstellt. Je mehr Humankapital in Form von Bildung im Forschungssektor bereits vorhanden ist, desto mehr neue Ergebnisse können erzielt werden, wodurch letztlich die Produkt- und Produktionsanlagenvielfalt in einer Volkswirtschaft bestimmt wird. ${ }^{22}$

Nach Ansicht von Barro und Sala-i-Martin (1997) ist es wiederum notwendig, zwischen technischem Fortschritt auf der einen und der Adaption von bereits bestehenden Technologien auf der anderen Seite zu unterscheiden. Für die tech-

\footnotetext{
${ }^{22}$ Wäre dieser Zusammenhang unterproportional, würde die Wachstumsrate des Wissens gegen Null gehen und damit auch die Wachstumsrate des Einkommens. Ein überproportionaler Einfluss des bereits existierenden Wissens würde hingegen dazu führen, dass sowohl das neue Wissen als auch der Output in endlicher Zeit gegen unendlich gehen, so dass die Annahme der Linearität hier als die einzig sinnvolle erscheint (vgl. Solow (2000), S. 152).
} 
nologische Entwicklung gilt allgemein, dass theoretisch eine unendlich große Zahl noch nicht genutzter Ideen existiert und dass damit auch die Zahl noch möglicher Erfindungen unbegrenzt ist. In diesem Zusammenhang ist es daher möglich, dass bereits akkumuliertes Wissen zukünftige Innovationen sogar noch beschleunigen könnte. Die Skalenerträge können gemäß dieser Argumentation als zumindest konstant angenommen werden.

Fallende Skalenerträge lassen sich insbesondere dann begründen, wenn nicht Innovationen im Vordergrund der Betrachtung stehen, sondern die Fähigkeit von Unternehmen, Imitationen durchzuführen. Letztere unterscheiden sich von Innovationen dadurch, dass hier die Zahl der verfügbaren Güter begrenzt ist. Befindet sich eine Ökonomie zunächst weit entfernt von der Wissensgrenze, existiert bereits eine hohe Zahl kopierbarer Güter. Die Nachahmung dieser Güter ist mit relativ geringen Imitationskosten verbunden, sobald die Produktion mit dem jeweiligen Wissensstand der Ökonomie durchführbar ist. Aufgrund der hohen Zahl imitierbarer Güter läuft der Imitationsprozess zunächst beschleunigt ab, so dass auch entsprechend hohe Wachstumsraten realisiert werden können, indem sich die Differenz zwischen dem maximal möglichen und dem tatsächlich erreichten Technologieniveau im Zeitablauf verringert. Je weiter dieser Prozess allerdings fortgeschritten ist, desto schwieriger wird es, neue Technologien zu adaptieren, da diese in ihrer Herstellung komplexer werden. Es dauert länger, bis Fortschritte in der Produktion zu erzielen sind, was wiederum mit sinkenden Wachstumsraten einhergeht.

Dass sich Länder mit identischer Humankapitalausstattung teilweise dennoch unterschiedlichen Wachstumsraten gegenübersehen, lässt sich über die Orientierung an verschiedenen Führungsnationen begründen. Hier kann sowohl die räumliche Entfernung zwischen den Ländern als auch die politische Ausrichtung des jeweiligen Landes eine Rolle spielen.

Den Überlegungen von Barro und Sala-i-Martin (1997) zufolge stehen Wachstumsmodelle, die konstante Skalenerträge in der Forschung unterstellen, nicht grundsätzlich in einem Widerspruch zu Modellen mit abnehmenden Skalenerträgen, da letztere weniger die Grundlagenforschung reicherer Länder als vielmehr den Aufholprozess ärmerer Länder zum Gegenstand haben.

Ein anderes Argument für die theoretische Koexistenz fallender sowie konstanter Skalenerträge liefern Blackburn et al. (2000): Betrachtet man einzig den Forschungs- und Entwicklungssektor, so scheint eine mögliche Redundanz von Forschungsergebnissen nicht ausgeschlossen, weshalb eine Verdopplung des Forschungsetats nicht zwangsläufig zu einer Verdopplung des Outputs führt. Diese Überlegung stützt daher zunächst die Annahmen von Romer (1986). 
Eine mögliche Begründung für konstante oder sogar steigende Skalenerträge liefert hingegen die Fähigkeit, bereits akkumuliertes Wissen zu einem Großteil an die nachfolgende Generation weiterzugeben, so dass qualitativ gleichwertige Forschungsergebnisse in der Zukunft mit gleich viel oder weniger Personal- und Zeitaufwand erreicht werden können. Somit wäre zudem der Bildungskapitalbestand innerhalb einer Ökonomie für die Effizienz, mit der Forschung betrieben und Entwicklungen durchgeführt werden können, entscheidend (vgl. Blackburn et al. (2000), S. 189).

\section{Produktion vs. Forschung und Entwicklung}

Das vierte Unterscheidungskriterium stellt auf die Frage ab, in welchem Sektor das akkumulierte Humankapital maßgeblich eingesetzt wird. Lucas (1988) berücksichtigt in seinem Wachstumsmodell die Akkumulation von Bildungskapital unter Berücksichtigung der optimalen Zeitallokation. Damit wird die effektive Arbeitskraft im Produktionsprozess nicht mehr nur durch das Produkt aus Arbeitskräften und Zeit beschrieben, sondern sie enthält darüber hinaus eine Gewichtung mit den individuellen Fähigkeiten. Durch diese Vorgehensweise wird neben der quantitativen Arbeitskraft auch der Qualität der Arbeit Rechnung getragen und somit endogenes Wachstum erzeugt. ${ }^{23}$

Über die Arbeit von Lucas hinaus geht der Ansatz von Romer (1990), in dem Bildung zusätzlich zum Gütersektor auch in Forschung und Entwicklung eingesetzt wird. Den maßgeblichen Unterschied zwischen diesen Sektoren bildet die Annahme, dass physische Arbeitskraft im Forschungssektor ohne Verwendung bleibt, weshalb dieser ausschließlich bildungsintensiv ist. Das generierte Wissen wird wiederum in der Produktion eingesetzt und führt so $\mathrm{zu}$ Wachstum. Auch wenn Romer annimmt, dass das insgesamt verfügbare Bildungskapital kurzfristig konstant ist und sich lediglich die Aufteilung zwischen den Sektoren ändern kann, ist die Bildung hier die entscheidende Skalenvariable, da sie die Ergebnisse im Forschungssektor maßgeblich determiniert (vgl. Romer (1990), S. 95). Endogenes Wachstum lässt sich in diesem Modell allein über den Anteil des Bildungskapitals im Forschungssektor im Verhältnis zum Gütersektor begründen (vgl. Solow (2000), S. 153).

23 Solow (2000) führt das bei Lucas generierte endogene Wachstum hingegen hauptsăchlich auf die Modellannahmen zurück: Freizeit ist in dem Modell von Lucas exogen gegeben, so dass die Zeitallokation nur die Arbeitszeit und den Zeitaufwand für Bildungsinvestitionen betrifft. Wird Freizeit endogen bestimmt, ergibt sich eine gleichgewichtige Wachstumsrate in Hobe des exogenen technischen Fortschritts aus dem neoklassischen Modell von Solow (1956), so dass sich endogenes Wirtschaftswachstum entsprechend nicht über die Akkumulation von Bildungskapital erreichen lässt. 


\section{Positive externe Effekte}

Das letzte Unterscheidungsmerkmal, das im Rahmen dieser Arbeit behandelt wird, sind positive externer Effekte. Romer (1986) begründet das Vorliegen derartiger Effekte mit der Annahme, dass die Nutzung neuen Wissens durch konkurrierende Unternehmen in den meisten Fällen nicht vollständig ausgeschlossen werden kann. Die Anmeldung einer Erfindung zum Patent schützt demnach zwar vor einer Nachahmung des gleichen Produkts oder Herstellungsverfahrens, nicht aber vor einer Weiterentwicklung der gewonnen Erkenntnisse im Rahmen eigener Forschungstätigkeiten. Eine vollständige Internalisierung der externen Effekte findet folglich nicht statt.

Letztlich implizieren positive externe Effekte zu geringe Wachstumsraten, da Unternehmen zu wenig in die Forschung investieren. Blieben sämtliche Unternehmen von den Externalitäten unbeeinflusst, könnten auch alle hiervon profitieren, so dass es zu einer Pareto-Verbesserung käme, die mit höheren Wachstumsraten einherginge. Tatsächlich unterliegt jedes Unternehmen jedoch dem Anreiz, seine eigenen Forschungsbemühungen so gering wie möglich zu halten und so in stärkerem Maße an den übrigen Unternehmenserfolgen zu partizipieren, ohne selbst einen Beitrag geleistet zu haben.

Positive externe Effekte und damit Unterschiede zwischen optimalen und gleichgewichtigen Wachstumsraten sind auch Gegenstand der Arbeit von Lucas (1988). ${ }^{24}$ Im Gegensatz zu Romer (1986) bezieht Lucas das Vorliegen von Externalitäten nicht auf die Forschungstätigkeit von Unternehmen, sondern insbesondere auf die Akkumulation von Bildungskapital durch die einzelnen Individuen. Die Auswirkungen der Bildung auf die individuelle Produktivität werden direkt über den Lohn abgegolten, was Lucas als internen Effekt bezeichnet. Der positive externe Effekt ergibt sich hier aus dem durchschnittlichen Bildungsniveau, das sich positiv auf die gesamte Faktorproduktivität auswirkt.

Um eine Externalität handelt es sich hierbei insofern, als dass kein Individuum dieses durchschnittliche Bildungsniveau in seiner Zeitallokationsentscheidung berücksichtigt, da der Effekt eines Einzelnen auf den Durchschnitt verschwindend gering ist (vgl. Lucas (1988), S. 18). Damit wird zumindest aus gesamtwirtschaftlicher Sicht zu wenig Zeit in Bildung investiert, weshalb physisches Kapital im Lucas-Modell mit einer höheren Rate wächst als Bildungskapital. Im

\footnotetext{
${ }^{24}$ Die Formulierung des Modells ist angelehnt an die Arbeit von Uzawa (1965). Uzawa unterstellt konstante Skalenerträge, die Produktionstechnologie des Bildungskapitals ist linear. Unter diesen Annahmen ist unbegrenztes Wirtschaftswachstum möglich.
} 
Umkehrschluss würden weitere Bildungsinvestitionen einen Anstieg des ProKopf-Konsums ermöglichen. ${ }^{25}$

Romer (1990) schreibt wiederum nicht den individuell erworbenen Fähigkeiten, sondern dem öffentlich in der Gesellschaft verfügbaren Wissen eine für das Wachstum entscheidende Rolle zu, indem sich hieraus ebenfalls positive externe Effekte ergeben. Der wesentliche Unterschied zu der Arbeit von Lucas (1988) besteht in der Interpretation von technischem Fortschritt im Sinne eines öffentlichen Gutes. Während persönliche Fähigkeiten direkt mit dem betreffenden Individuum verbunden und daher gesamtwirtschaftlich nur begrenzt nutzbar ist, ist neues Wissen dauerhaft verfügbar, sobald es öffentlich zugänglich gemacht wurde. Darüber hinaus ist es im Gegensatz zum individuellen Bildungskapital nicht rival, sondern kann von jedem Individuum gleichermaßen genutzt werden, woraus sich Wissensspillover ergeben können (vgl. Romer (1990), S. S75). Sowohl innerhalb eines Unternehmens als auch gesellschaftlich können sich so zudem steigende Skalenerträge aus der Forschung ergeben.

Dieser Überblick über die modelltheoretischen Unterschiede in der wachstumstheoretischen Literatur gibt einen kurzen Einblick in die kontroverse Diskussion über die Determinanten des Wirtschaftswachstums. Ein wesentliches Problem besteht darin, dass technischer Fortschritt nicht prognostizierbar ist und keiner Technologie folgt. Damit kann nicht eindeutig festgestellt werden, ob eine Ausweitung der Forschung das neue Wissen über- oder unterproportional erhöht oder ob ein linearer Zusammenhang existiert. Ebenfalls unsicher ist, ob die Akkumulation von Bildungskapital für das Wachstum entscheidend ist (siehe hierzu die Arbeit von Lucas (1988)) oder ob der Bildungskapitalbestand die entscheidende Variable darstellt (vgl. Romer (1990)).

\subsubsection{Empirische Überprüfung des Bildungskapitalansatzes}

Neben den theoretischen Ansätzen existiert eine große Zahl empirischer Arbejten, die den Einfluss von Bildung auf das Wirtschaftswachstum zum Gegenstand haben. Hier stellt sich die Frage, ob Bildung tatsächlich die endogene Wachstumstheorie stützt, oder ob vielmehr die Konvergenzhypothese des Solow-Modells bestätigt werden kann, wonach in der Anfangsausstattung identische Staaten zu einem einheitlichen Gleichgewicht konvergieren.

${ }^{25}$ Analog zu dem Modell von Romer (1986) führt eine hohe Gegenwartspräferenz auch bei Lucas (1988) zu Unterinvestitionen in die Bildung, weshalb wirtschaftspolitische Maßnahmen, die eine Senkung des gegenwärtigen Konsumniveaus nach sich ziehen, zu einer verstärkten Forschungstätigkeit und damit zu mehr Wirtschaftswachstum fuhren. 
Eine Bestätigung der Konvergenzhypothese und damit der neoklassischen Wachstumstheorie findet sich beispielsweise bei Mankiw et al. (1992) (hiernach MRW). In dieser empirischen Arbeit wird die Schulbesuchsquote (,school enrolment rate") als Variable für Investitionen in die Bildung verwendet. Nach Ansicht von MRW erklären Unterschiede in den Ersparnissen, dem Bevölkerungswachstum und der Bildung die Divergenzen in den Pro-Kopf-Einkommen zwischen den Ländern. Weiterhin zeigt sich, dass Bildung die Konvergenzgeschwindigkeit positiv beeinflusst, während die Bedeutung des Bevölkerungswachstums sowie der Sparquote zurückgeht.

Hinsichtlich der in der endogenen Wachstumstheorie getroffenen Annahme konstanter Skalenerträge zeigt sich zudem, dass diese Überlegung einer empirischen Überprüfung nicht standhält. Vielmehr lassen die Ergebnisse auf abnehmende Skalenerträge schließen, da eine zusätzliche Bildungseinheit den Lohn in Entwicklungsländern stärker steigen lässt als in Industrienationen. Problematisch ist im Hinblick auf die verwendete Bildungsvariable allerdings die fehlende $\mathrm{Ab}$ grenzung zwischen Bildung als Bestands- und als Stromgröße. Die Schulbesuchsquote wird in einigen empirischen Arbeiten als Indikator für den Humankapitalbestand verwendet, während sie in anderen Arbeiten gerade als Indikator für die Akkumulation dient (vgl. Gemmell (1995), S. 169f.).

Barro und Lee (2000) geben darüber hinaus zu bedenken, dass die Zahl der Schuljahre keinen Aufschluss darüber gibt, welche Fähigkeiten Individuen anschließend erworben haben, da beispielsweise Qualitätsunterschiede zwischen Schulen verschiedener Länder sind nicht über die Schulbesuchsquote abgebildet werden können. Wird die Qualität hingegen über Testergebnisse, beispielsweise im Bereich der Mathematik, geprüft, so ist diese zwar vergleichbar, Verzerrungen aufgrund von Missverständnissen bei der Auslegung der Fragen können jedoch ebenfalls nicht ausgeschlossen werden.

Die Ergebnisse von MRW erscheinen in diesem Zusammenhang nicht weiter verwunderlich. Werden ausschließlich der Schulbesuch sowie die Veränderung des Schulbesuchs als Indikatoren für das Bildungskapital verwendet, ergeben sich hier ex definitionem Aufholprozesse für Entwicklungsländer: In Ländern mit historisch niedriger Schulbesuchsquote ist eine Steigerung problemlos möglich, es erfolgt hier automatisch eine Anpassung an Industrieländer, in denen diese Quote bereits sehr hoch ist und Zuwächse kaum noch erreicht werden können. So zeigt beispielsweise die Debatte über zu lange Schulzeiten in Deutschland, dass ein langer Schulbesuch nicht zwangsläufig eine verbesserte Wettbewerbsfähigkeit der Individuen mit sich bringen muss, sondern dass vielmehr der gelernte Stoff innerhalb eines bestimmten Zeitfensters für den Erfolg maßgeblich ist. 
Dieses Ergebnis stützt vielmehr die Theorie von Nelson und Phelps (1966), wonach bereits existierende Technologien durch den erweiterten Schulbesuch standardisiert werden können. Insofern ist hier ein Konvergenzprozess zu beobachten, der sich sowohl auf die Allgemeinbildung als auch auf das Einkommen bezieht.

In der Schulbesuchsquote ebenfalls nicht enthalten ist die so genannte tertiäre Ausbildung, die aufgrund des Zusammenhangs mit der Forschungstätigkeit insbesondere in entwickelten Ländern zu berücksichtigen ist. Diese Form der Ausbildung geht über den Pflichtschulbesuch hinaus, indem sie die gesamte weiterführende Ausbildung, insbesondere jedoch das Studium, umfasst. Während der normale Schulbesuch die Verbreitung von Allgemeinbildung zum Ziel hat, werden im Rahmen eines Studiums spezielle Kenntnisse vermittelt, welche in der Forschung und Entwicklung weiter verwendet werden können. Aus diesem Grund steht die Arbeit von MRW zumindest nicht zu dem theoretischen Konzept von Romer (1990) im Widerspruch. Eine Erweiterung der verwendeten Daten von MRW um entsprechende Bildungsdaten wäre dementsprechend notwendig, um das Solow-Modell gegen andere Definitionen von Bildung zu testen.

Nach Ansicht von Grossman und Helpman (1994) sind die empirischen Ergebnisse von MRW erstens auf die bestehenden Unterschiede zwischen armen und reichen Ländern zurückzuführen. Werden hingegen nur die OECD-Länder betrachtet, nimmt der Erklärungsgehalt des Solow-Modells deutlich ab. Zweitens kritisieren die Autoren, dass der technische Fortschritt in der Arbeit von MRW für alle Länder identisch ist, obwohl sich die Wachstumsraten der Faktorproduktivitäten zwischen den Nationen unterscheiden. Zum dritten sind die Wachstumsraten des technischen Fortschritts sowie des physischen Kapitals in der Arbeit von MRW voneinander unabhängig. ${ }^{26}$ Durch technischen Fortschritt kann aber auch die Produktivität des Kapitals erhöht werden, woraus wiederum neue Investitionen resultieren können. Die Annahme der vollständigen Unabhängigkeit der Produktionsfaktoren wäre damit nicht weiter haltbar. Es stellt sich aus diesen Gründen die Frage, ob die Ansätze der endogenen Wachstumstheorie einen höheren Erklärungsgehalt für das Entstehen von Wirtschaftswachstum liefern.

${ }^{26}$ Bezeichnet $Y$ den Output, $A$ den technischen Fortschritt, $K$ das eingesetzte Kapital, $L$ die eingesetzte Arbeitskraft und $g$ die Wachstumsraten, dann folgt aus einer Einkommensgleichung der Form $Y=A K^{\alpha} L^{\beta}$ für die Wachstumsraten: $g_{Y}=g_{A}+\alpha g_{K}+\beta g_{L}$. Aufgrund der Additivităt der einzelnen Wachstumsraten sind diese voneinander unabhăngig. 
Um diese Fragestellung zu berücksichtigen, gehen Grossman und Helpman (1994) davon aus, dass die Forschungstätigkeit der Unternehmen als endogen betrachtet werden muss. Grundlage hierfür ist die Annahme, dass die Wahrscheinlichkeit, die nächste Generation eines bestimmten Produktes zu erfinden, proportional zu der in der Forschung eingesetzten Arbeitskraft ist. Unternehmen wetteifern um die nächste Produktgeneration, die Dauer der Forschungstätigkeit bis hin zu einer marktfähigen Innovation ist jedoch zufällig und variabel zwischen den einzelnen Sektoren.

Auf individueller Ebene kann das Wissen nicht ohne Grenzen wachsen, da dieses direkt an Individuen gebunden und damit auf die Lebensdauer beschränkt ist. Führt das begrenzte individuelle Wissen jedoch zu neuen Produkten oder wird es anderweitig verbreitet, kann auf dieser Basis unbegrenzt neues Wissen akkumuliert werden. Während der technische Fortschritt damit auf der Mikroebene stochastisch ist, ergibt sich aggregiert eine gleichgewichtige Innovationsgeschwindigkeit. Die tatsächliche Geschwindigkeit des langfristigen Wachstums hängt in diesem Modellansatz von den Kosten und Erträgen der Forschung ab. ${ }^{27}$ Ein Anstieg in der Profitabilität von Forschung und Entwicklung kann damit eine Verschiebung der Ressourcen zugunsten des Forschungssektors nach sich ziehen. ${ }^{28}$ Aus diesem Grund können sich auch die Wachstumsraten von Ländern unterscheiden, die über eine identische Einwohnerzahl verfügen, da nicht nur die insgesamt verfügbare Arbeitskraft eine Rolle spielt, sondern auch das hiermit verbundene Bildungskapital. Je mehr Humankapital in Form von Bildung zur Verfügung steht, desto schneller wächst die Ökonomie, solange ein Teil in der Forschung eingesetzt wird und somit die Innovationstätigkeit vorantreibt, woraus sich nachhaltiges Wachstum ergibt (vgl. Grossman und Helpman (1994), S. 35f.).

Im Rahmen der theoretischen Diskussion über fallende oder konstante Skalenerträge wurde bereits angemerkt, dass Unterschiede in der Ausgestaltung des technischen Fortschritts zwischen Ländern mit niedrigem und solchen mit hohem Einkommen existieren können. Benhabib und Spiegel (1994) stellen die

\footnotetext{
${ }^{27}$ In der Empirie werden die direkten Ausgaben für Forschung und Entwicklung, beispielsweise die Lohn- und Materialkosten einer Forschungsabteilung, als Indikator für den Forschungsaufwand verwendet. Für vollständig neue Produktgenerationen mag diese Vorgehensweise zutreffend sein, darüber hinaus können Weiterentwicklungen und Verbesserungen von bereits existierenden Produktgenerationen jedoch auch aus dem laufenden Produktionsprozess heraus entstehen. Diese Form der indirekten Forschung durch die Erfahrung der Arbeitskräfte wird nicht empirisch erfasst (vgl. Grossman und Helpman (1994), S. 31).

${ }^{28}$ Eine Steigerung der Erträge ist insbesondere auf Märkten mit unvollkommenem Wettbewerb möglich, da hier die Produkte zu einem Preis oberhalb der entstandenen Kosten verkauft werden können.
} 
Hypothese auf, dass die Innovationstätigkeit innerhalb eines Landes mit dem Bildungskapital ansteigt, da die Produktivität in der Forschung steigt somit die Kapazität für Innovationen ausgeweitet wird (vgl. hierzu den theoretischen Ansatz von Romer (1990)). Eine zweite Hypothese bezieht sich auf die Geschwindigkeit des technologischen Aufholprozesses und die Verbreitung von Technologien, welche durch das Bildungsniveau positiv beeinflusst werden (siehe hierzu Nelson und Phelps (1966)).

Hieraus leitet sich die Überlegung ab, dass unterschiedliche Bildungskapitalbestände auch zu Unterschieden in den Wachstumsraten führen. So wird das Land mit dem höchsten Bildungsstand die Führungsposition übernehmen und so lange innehaben, wie gegenüber den Verfolgernationen ein Vorsprung im Bildungskapital vorhanden ist. Bildlich gesprochen vergleichen Benhabib und Spiegel die anfuhrende Nation mit einer Lokomotive: „In the long run, the country with the highest level of $H$ [Human Capital] acts as the 'locomotive' of growth by expanding the set of attainable knowledge, pulling all others along through the catch-up effect, and all countries grow at the same rate" (Benhabib und Spiegel (1994), S. 157). Im Umkehrschluss bedeutet diese Überlegung, dass es zu einem Aufholprozess ärmerer Länder kommen kann, sobald diese über ein entsprechend hohes Bildungsniveau verfugen. Solange jedoch Unterschiede in den Bildungsniveaus bestehen, kann auch der Übergang zu einer gleichgewichtigen Wachstumsrate nicht abgeschlossen werden.

Auf Basis dieser Überlegungen lassen sich damit auch die hohen Wachstumsraten ärmerer Länder erklären, da diese neue Technologien lediglich adaptieren, nicht aber in eigene Forschungstätigkeit investieren müssen. In Ländern mit niedrigem Pro-Kopf-Einkommen spielt die Bildung empirisch daher keine primäre Rolle als Wachstumsmotor, sondern sie unterstützt lediglich den Imitationsprozess. Während die genannten Effekte für Länder mittlerer Einkommen nicht separiert werden können, kann der Einfluss der Bildung für reichere Länder wiederum empirisch nachgewiesen werden. Hierdurch wird die Hypothese bestätigt, dass reichere Länder den Aufholprozess zur anführenden Nation nahezu abgeschlossen haben und somit selbst in die Forschung investieren müssen. Aus diesen Überlegungen folgt weiterhin, dass die Bedeutung des Bildungskapitals für das Wirtschaftswachstum nur dann unverzerrt abgebildet werden kann, wenn der Aufholprozess bei der empirischen Überprüfung explizit berücksichtigt wird. Aus diesem Grund wird das Ausgangseinkommen bei Benhabib und Spiegel als Kontrollvariable in der empirischen Untersuchung berücksichtigt.

Die Ergebnisse von Benhabib und Spiegel (1994) werden von Papageorgiou (2003) nochmals empirisch untersucht. Ausgangspunkt ist die Hypothese, dass Grundschulbildung zwar die Produktivität der Outputproduktion erhöht, jedoch 
ohne Einfluss auf Forschung und Entwicklung ist. Für die weiterführende Bildung ist das Gegenteil der Fall, d. h. diese erhöht insbesondere die Effizienz im Forschungssektor (vgl. Papageorgiou (2003), S. 623). ${ }^{29}$

Zur Überprüfung dieser Hypothese wird der Einfluss der Bildung auf das Wachstum für reiche, mittlere und arme Länder getrennt geschätzt. ${ }^{30}$ Für das obere Drittel ist die Akkumulation von Bildungskapital im Outputsektor nicht relevant, vielmehr profitieren diese Nationen vom Einsatz der Bildung insbesondere im Bereich der Innovation, aber auch bei der Nachahmung von bereits bestehenden Gütern und Technologien. Für Länder mittleren Einkommens kann lediglich eine Tendenz zur Imitation festgestellt werden. Weder das Bildungskapitalwachstum in der Outputproduktion noch die Innovationskomponente sind statistisch signifikant. Während die körperliche Arbeitskraft für diese beiden Ländergruppen keine wesentliche Rolle spielt, ist sie mit ausschlaggebend für das Wirtschaftswachstum der armen Länder. Bildungskapital wird als Input in der Güterproduktion verwendet, so dass das untere Drittel der Länder insbesondere von einem Anstieg in der Grundschulbildung profitiert. Wichtig für das Wirtschaftswachstum ist darüber hinaus die Fähigkeit, vorangegangene Entwicklungen anderer Länder zu imitieren.

Diese Ergebnisse lassen den Schluss zu, dass Bildungskapital je nach Wohlstand des Landes unterschiedlich eingesetzt wird. Ist die Wirtschaft des Landes durch einen großen primären und sekundären Sektor gekennzeichnet, spielt insbesondere die grundlegende Bildung der gesamten Bevölkerung eine Rolle für die weitere Entwicklung des Landes. Forschung und Entwicklung haben in diesem Stadium noch keinen Einfluss auf das Wirtschaftswachstum, da die Zahl der möglichen Anwender der Forschungsergebnisse noch zu gering ist. Forschung lohnt sich demnach erst mit steigendem Einkommen, wenn die Menge der noch nicht imitierten Güter zurückgeht. ${ }^{31}$

${ }^{29}$ Messprobleme können insbesondere bei dem Vergleich unterschiedlicher Schulsysteme auftreten. Hier wird ,primary education“ mit Grundschulbildung und ,post-primary education" mit weiterführende Bildung übersetzt.

${ }^{30}$ Die Gesamtstichprobe umfasst 80 Nationen, die Aufteilung in drei gleich große Unterstichproben erfolgt nach dem Pro Kopf-BIP von 1960. Untersucht wird das Wirtschaftswachstum bis zum Jahr 1987.

${ }^{31}$ Bernanke und Gürkaynak (2001) widerlegen die Ansätze des Modells von Solow (1956) empirisch, indem ebenfalls der Ansatz von Mankiw et al. (1992) verwendet wird. Die Autoren zeigen, dass das Solow-Modell nicht mit den verfügbaren Daten übereinstimmt, weshalb Wirtschaftswachstum hierüber nicht hinreichend erklärt wird. Auf der anderen Seite kann aber auch die endogene Wachstumstheorie nicht bestätigt werden. Die Untersuchung kommt zu dem Ergebnis bedingter Konvergenz in dem Sinne, dass Länder mit geringem Einkommen 
Weitere empirische Ergebnisse hinsichtlich der Determinanten ökonomischer Wachstumsraten finden sich zudem bei Barro und Sala-i-Martin (1995). Hier ergibt sich für Männer ein hoch signifikanter Zusammenhang auf dem 1\%-Niveau für die weiterführende Bildung, während der Einfluss der höheren Bildung lediglich auf dem 10\%-Niveau signifikant ist.

In einer weiteren Studie quantifizieren Barro und Sala-i-Martin (1995) die genauen Effekte der Bildung auf die Wachstumsraten. So bringt eine Erhöhung der durchschnittlichen Bildungsdauer bezüglich weiterführender Bildung um 0,68 Jahre einen Anstieg der Wachstumsrate um 1,1 Prozentpunkte pro Jahr mit sich. Eine Ausweitung der für höhere Bildung verbrachten Zeit um 0,12 Jahre erhöht die Wachstumsrate zusätzlich um 0,5 Prozentpunkte pro Jahr (vgl. Barro und Sala-i-Martin 1995, S. 431). ${ }^{32}$

Barro (1997) hat den Einfluss der Bildung unter Verwendung derselben Variablen erneut untersucht mit dem Ergebnis, dass die Grundschulausbildung von Männern lediglich einen insignifikanten Effekt auf das Wachstum ausübt. Dennoch ist der Einfluss der Grundschulbildung nicht zu vernachlässigen, da diese die Voraussetzungen für effiziente Weiterbildung schafft, deren Einfluss auf die Wachstumsrate wiederum signifikant ist (vgl. Barro (1997), S. 19f.).

Die Analyse der einzelnen Arbeiten zeigt die vielfältigen Wirkungskanäle der Bildung im Hinblick auf das Wachstum. Zusammenfassend ist zu konstatieren, dass Allgemeinbildung insbesondere in unterentwickelten Ökonomien von Bedeutung ist, da hierdurch die Arbeitsproduktivität gesteigert und die Adaption technischer Entwicklungen beschleunigt wird. Hingegen spielt für Länder, die sich an der Technologiegrenze befinden, insbesondere die höhere Bildung eine besondere Rolle, da diese elementar für erfolgreiche Forschung benötigt wird.

\subsection{Gesundheit als Produktionsfaktor}

\subsubsection{Grundlegende Zusammenhänge}

Die Überlegung, dass neben der Bildung auch die Gesundheit eine große Rolle für die wirtschaftliche Entwicklung spielt, geht insbesondere auf Schultz (1961) und Mushkin (1962) zurück. Eine bedeutende Rolle in der wachstumstheoreti-

hohe Wachstumsraten erzielen können, bis sie ihre Steady State-Wachstumsraten erreichen. Diese müssen nicht mit den gleichgewichtigen Raten der fuhrenden Nationen übereinstimmen. Die endogene Wachstumstheorie prophezeit hingegen keine bedingte Konvergenz.

${ }^{32}$ Im Hinblick auf die weiterfuhrende Bildung von Frauen wird der positive Zusammenhang jedoch nicht eindeutig empirisch gestützt, vielmehr ergibt sich hier sogar ein negatives Vorzeichen des Koeffizienten. 
schen Literatur spielt die Gesundheit allerdings erst seit Beginn der 1990er Jahre, indem Mankiw et al. (1992) Gesundheit als eigenständigen Produktionsfaktor berücksichtigen.

Der Weltentwicklungsbericht der Weltbank fasst die Notwendigkeit einer Verbesserung der Gesundheit wie folgt zusammen:

„Improved health contributes to economic growth in four ways: it reduces production losses caused by worker illness; it permits the use of natural resources that had been totally or nearly inaccessible because of disease; it increases the enrolment of children in school and makes them better able to learn; and it frees for alternative uses resources that would otherwise have to be spent on treating illnesses." (World Bank (1993), S. 17).

Diese Zusammenfassung gibt nur einen kleinen Überblick über die zahlreichen Wachstumseffekte, die mit einer guten Gesundheit verbunden sind. Zudem lassen sich die Gesundheitseinflüsse in direkte Effekte, d. h. Wachstumseffekte, die sich aus einer gesteigerten Gesundheit per se ergeben, sowie indirekte Effekte unterscheiden, wobei bei letzteren die übrigen Produktionsfaktoren ebenfalls durch die Gesundheit beeinflusst werden.

Direkte Auswirkungen ergeben sich insbesondere durch den Einfluss der Gesundheit auf die Produktivität der Erwerbstätigen sowie durch geringere Fehlzeiten. Individuen mit schlechter Gesundheit verfügen über eine stark begrenzte körperliche Leistungsfähigkeit, so dass für ein bestimmtes Arbeitspensum eine längere Zeit benötigt wird. Gleichzeitig führen Erkrankungen zu einer geringeren Konzentrationsfähigkeit, wodurch nicht nur die Quantität, sondern auch die Qualität der verrichteten Arbeit gemindert wird. Während sich ein schlechter Gesundheitszustand bei abhängig beschäftigten Individuen über eine geringere Produktivität dauerhaft im Lohn niederschlägt, bedeutet dies für Selbständige eine besondere Gefährdung der Lebensgrundlage. Zudem sind hauptsächlich Personen betroffen, die ausschließlich körperliche Arbeit verrichten, so dass insbesondere Personen mit niedrigen Einkommen größeren Gesundheits- und damit Einkommensrisiken ausgesetzt sind.

Neben diesem direkten Effekt, der sich positiv auf die effektive Arbeitskraft auswirkt, ergeben sich zahlreiche indirekte Effekte, indem die Akkumulation von physischem Kapital gefördert wird und Anreize für Bildungsinvestitionen gesetzt werden, wodurch sich wiederum Produktivitätseffekte ergeben.

Der Einfluss auf die Kapitalakkumulation erfolgt erstens über die Lebenserwartung und die hiermit verbundenen Sparanreize. Während Personen, die ein langes Leben über die Erwerbstätigkeit hinaus erwarten, einen Teil des gegenwärti- 
gen Einkommens für die Zukunft sparen und somit Investitionen fördern, ist bei Individuen mit geringerer Lebenserwartung die Gegenwartspräferenz höher, so dass letztlich mehr konsumiert und weniger physisches Kapital akkumuliert wird. Zweitens sind die Anreize zu Investitionen insbesondere dann gering, wenn diese zum Humankapital komplementär sind. Ein hoher Krankenstand sowie eine geringe Lebenserwartung senken die Rendite von Unternehmen besonders, wenn qualifizierte Arbeitskräfte benötigt werden, da diese schwerer zu ersetzen sind. Folglich werden sich derartige Unternehmen eher in Ländern mit einem entsprechend hohen Gesundheitsniveau ansiedeln. Drittens schließlich wird nicht nur die private Kapitalakkumulation beeinflusst, sondern auch die Möglichkeit des Staates, eine angemessene Infrastruktur bereitzustellen. Die permanente Verbreitung von Krankheiten bindet einen Teil der verfügbaren Ressourcen für kurative Zwecke, so dass diese nicht für staatliche Investitionen zur Verfügung stehen (vgl. Sala-i-Martin (2005), S. 102f.). ${ }^{33}$

Die indirekten Einflüsse der Gesundheit auf das Wirtschaftswachstum, die sich aus der Bedeutung der Gesundheit für die Bildung ableiten, sind ungleich zahlreicher und werden nachfolgend noch ausführlicher behandelt. Insgesamt sind hier fünf Wirkungskanäle zu nennen, von denen vier unmittelbar die Schulausbildung von Kindern betreffen und einer sich auf die Anreize zu betrieblicher Weiterbildung bezieht.

Zunächst führt häufige Krankheit bei Kindern zu vermehrten Fehlzeiten in der Schule sowie zu einer geringeren Konzentrationsfähigkeit. Durch schlechtere Schulabschlüsse sind auch die zukünftigen Einstiegslöhne sowie die zu erwartenden Lohnsteigerungen geringer, so dass sich Gesundheitsprobleme in der Kindheit negativ auf das gesamte Lebenszeiteinkommen auswirken.

Der zweite Effekt bezieht sich auf die Möglichkeiten der Eltern, den Kindern eine entsprechende Schulbildung zukommen zu lassen. Eine hohe Kindersterblichkeit, wie sie insbesondere in Entwicklungsländern anzutreffen ist, zwingt die Eltern zu einer gesteigerten Fertilität, damit zumindest ein Teil der Kinder das Erwachsenenalter erreicht. Je höher jedoch die Zahl der Kinder ist, desto weniger Ressourcen stehen für deren Ausbildung zur Verfügung, weil das verfügbare Einkommen auf mehr Personen aufgeteilt werden muss. Infolgedessen besteht

${ }^{33}$ Als besonders dramatisch ist hier die steigende Zahl der AIDS-Infektionen in Schwarzafrika zu sehen, da hierdurch nicht nur ganze Familien gefährdet sind, sondern die Betroffenen daruber hinaus leichter an Krankheiten wie Diarrhöe und Tuberkulose erkranken und somit hohe Behandlungskosten verursachen, obwohl es noch keine direkte Heilungsmethode gegen die AIDS-Erkrankung gibt (vgl. World Bank (1993), S. 20). 
insbesondere für Kinder armer Familien keine Möglichkeit, dieser Armutsfalle durch eine gute Schulbildung zu entkommen.

Drittens bedeutet eine geringe Lebenserwartung, dass einer langen Ausbildungszeit lediglich eine kurze Erwerbsperiode gegenübersteht. In einem solchen Fall ist die Rendite aus den Bildungsinvestitionen entsprechend gering, weshalb kein hinreichender Anreiz zu Bildungsinvestitionen besteht. Je höher folglich die Lebenserwartung ist, desto mehr Zeit kann für die Aus- und Weiterbildung aufgewendet werden.

Der vierte Einfluss der Gesundheit auf das Bildungskapital ergibt sich aus den Anreizen von Unternehmen, in die Weiterbildung der Beschäftigten zu investieren. Die Begründung hierfür erfolgt analog zu der Problematik einer zu geringen Lebenserwartung, denn eine hohe Erkrankungs- oder sogar Sterbewahrscheinlichkeit senkt die Rendite der Weiterbildungsmaßnahmen, so dass Unternehmen vermehrt in die Ausbildung derjenigen Arbeitskräfte investieren, die über eine ausreichende Gesundheit verfügen. Auch wenn sich dieser Effekt insbesondere auf die Problematik in Entwicklungsländern bezieht, so lassen sich dennoch $\mathrm{Pa}$ rallelen zu entwickelten Nationen ziehen, da hier insbesondere chronische Krankheiten zu vermehrter Erwerbsunfähigkeit führen und somit die Investitionen ohne entsprechende Erträge bleiben.

Zuletzt spielt nicht nur die Gesundheit von Kindern, sondern auch die der Eltern eine Rolle. So leben allein in Afrika 14 Millionen Waisen, deren Eltern an AIDS gestorben sind (vgl. Sala-i-Martin (2005), S. 101). In Ermangelung sozialer Sicherungssysteme und Hilfseinrichtungen für die betroffenen Kinder sind diese häufig auf sich allein gestellt. Hier fehlt es sowohl an finanzieller als auch an moralischer Unterstützung der Kinder, so dass diese bereits früh erwerbstätig werden müssen und ohne die Möglichkeit einer Ausbildung aufwachsen.

\subsubsection{Wachstumstheoretische Modellansätze}

Analog zu den wachstumstheoretischen Modellen, in denen die Bildung explizit als Modellvariable enthalten ist, lassen sich die Wachstumseffekte der Gesundheit in verschiedene Kategorien einordnen (vgl. Abbildung 4). So ist zunächst zu unterscheiden, ob das Gesundheitskapital in dem betreffenden Modell exogen gegeben ist oder aber endogen bestimmt werden kann. Während der Einfluss der Gesundheit auf das Wachstum im ersten Fall auf Produktivitätseffekte zurückgeführt werden kann, wird im zweiten Fall der Gesundheitssektor explizit in den Überlegungen berücksichtigt. Die zweite Unterscheidung bezieht sich auf die Frage, welche Bevölkerungsgruppe am meisten von den bereitgestellten Gesundheitsgütern profitiert: die momentan Erwerbstätigen oder der inaktive Teil der Bevölkerung, also Erwerbsunfähige, Kinder und Rentner. Zum Dritten kön- 
nen die einzelnen Modelle dahingehend unterschieden werden, ob von der Gesundheit ausschließlich direkte Produktivitätseffekte ausgehen, oder ob zudem indirekte Effekte bei der theoretischen Modellierung berücksichtigt werden. Zuletzt können auch im Rahmen der Gesundheitsproduktion positive externe Effekte vorliegen.

Abbildung 4: Gesundheitsbezogene Modellannahmen der Wachstumstheorie

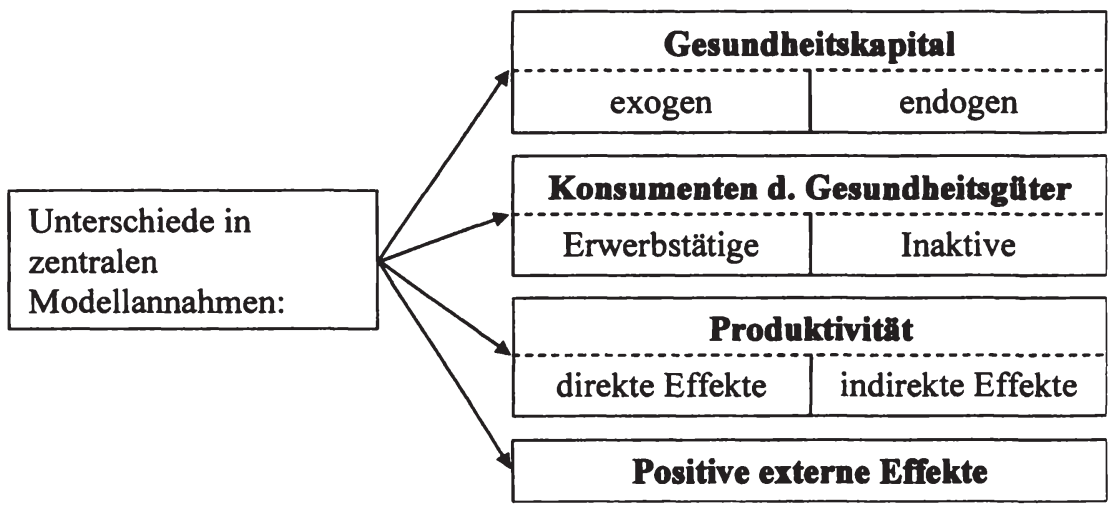

Quelle: Eigene Darstellung.

\section{Exogenes versus endogenes Gesundheitskapital}

Das erste Unterscheidungsmerkmal betrifft die Fragestellung, ob die Gesundheit der Bevölkerung exogen gegeben ist oder ob diese mit Hilfe von Investitionen beeinflusst werden kann. Für beide Fragestellungen gilt gleichermaßen, dass sich die Einwohner eines Landes nun in einen aktiven, erwerbstätigen und einen inaktiven Teil aufspalten. Zu der Gruppe der Inaktiven gehören neben Rentnern und Kindern auch diejenigen, die aufgrund von Krankheit erwerbsunfähig sind und damit ebenfalls keinen Beitrag zur Produktion leisten. Damit ist das effektive Arbeitsangebot in einer Volkswirtschaft nicht mehr durch die Bevölkerungszahl gegeben, sondern es ergibt sich ausschließlich aus dem Angebot derjenigen, die über ausreichend Gesundheit verfügen und somit erwerbstätig sein können.

Je besser nun der exogen gegebene Gesundheitskapitalstock ist, desto höher ist auch das effektive Arbeitsangebot, woraus sich positive Wachstumseffekte ergeben können. Im Umkehrschluss wird das Wachstum gebremst, je schlechter der durchschnittliche Gesundheitszustand ist, da die vorhandene Arbeitskraft nicht vollständig produktiv genutzt werden kann. Eine exogene Verbesserung der Gesundheit hätte damit den gleichen Effekt wie eine exogene Veränderung 
der Produktivität im Solow-Modell. Im Unterschied zu einer Produktivitätssteigerung ist eine Verbesserung der Gesundheit jedoch nur begrenzt möglich, da Gesundheit definitionsgemä $\beta$ ein Maximum (perfekte Gesundheit) besitzt. In einem Wachstumsmodell mit exogen gegebenem Gesundheitskapitalstock lassen sich daher keine dauerhaften Wachstumseffekte herleiten (vgl. Muysken et al. (2003)).

Denkbar und weitaus realistischer ist indessen die Möglichkeit, Gesundheit endogen zu bestimmen. Dies kann beispielsweise über die Implementierung eines Gesundheitssektors in die wachstumstheoretischen Modelle erfolgen (vgl. Van Zon und Muysken (2001), Muysken et al. (2003)). Ein Teil der verfügbaren Arbeitskraft sowie der verfügbaren Ressourcen wird nun im Gesundheitssektor eingesetzt, um die Gesundheit der Bevölkerung zu verbessern und so das Arbeitskräftepotenzial optimal auszuschöpfen. Gleichzeitig unterliegt der Gesundheitskapitalstock einer positiven Abschreibungsrate, wodurch der Bestand an Gesundheitskapital kontinuierlich gesenkt wird (vgl. Muysken et al. (2003), S. 193) ${ }^{34}$ Eine Gesundheitsschädigung kann beispielsweise dann auftreten, wenn Erwerbstätigkeit und das Streben nach Effizienz in den Produktionsprozessen zu Leistungsdruck und Stress führen, woraus letztlich Fehlzeiten und damit ein Verlust an effektiver Arbeitskraft resultieren können (vgl. Van Zon und Muysken (2001)). Bei Johansson und Löfgren (1995) geht dagegen die Produktion von Konsumgütern mit Umweltverschmutzung einher, woraus sich ebenfalls negative Gesundheitseffekte ableiten. Ohne Investitionen führten Abschreibungen folglich dazu, dass immer mehr Erwerbstätige erkrankten und somit arbeitsunfähig würden, so dass der durchschnittliche Gesundheitskapitalbestand der Gesellschaft stetig absinken würde.

Wird nun angenommen, dass Individuen einen Teil ihres Einkommens für Gesundheitsleistungen aufwenden müssen, ergibt sich hieraus ein Trade-off zwischen dem heutigen Konsum und dem Konsum in der Zukunft: Auf der einen Seite ergeben sich aus der verbesserten Gesundheit Produktivitätseffekte, die mit einem gesteigerten Lohn einhergehen und somit ceteris paribus einen positiven Beitrag zum zukünftigen Konsum leisten. Auf der anderen Seite muss in der Gegenwart auf Konsum verzichtet werden, da zumindest ein Teil des Einkommens für den Erhalt oder eine Verbesserung der Gesundheit aufzuwenden ist. Dieser zweite Effekt kann allerdings insofern abgeschwächt werden, als dass zukünftig weniger Kapital für das Kurieren von Krankheiten zur Verfügung stehen muss. Durch das hiermit verbundene Absinken der Sparquote kann der gegenwärtige Konsum entsprechend ausgeweitet werden.

\footnotetext{
${ }^{34} \mathrm{Zu}$ der näheren Wirkungsweise von Abschreibungen auf den individuellen Gesundheitskapitalstock siehe die Kapitel 4 und 5.
} 
Für die Unternehmen bedeutet eine Verbesserung der Gesundheit eine Steigerung der Arbeitsproduktivität, wodurch der Lohnsatz steigt. Bei konstantem Zins werden Investitionen in Realkapital finanziell attraktiver, woraus sich eine gesteigerte Kapitalakkumulation ergibt.

Auf gesamtwirtschaftlicher Ebene führt die Berücksichtigung des Gesundheitssektors dazu, dass mit dem effektiven Arbeitsangebot zwar ein Produktionsfaktor in größerem Umfang zur Verfügung steht, darüber hinaus aber auch Ressourcen gebunden werden, die nicht für übrige Verwendungszwecke, beispielsweise die Güterproduktion oder die Akkumulation von Bildungskapital, zur Verfügung stehen. Die Effekte eines hohen Beschäftigungsgrads im Gesundheitssektor sind damit ambivalent: Liegen Ineffizienzen in der Produktion von Gesundheitskapital vor, werden die Produktionsfaktoren nicht optimal ausgelastet, wodurch sich negative Konsequenzen für das Wirtschaftswachstum ergeben. Diesem Effekt stehen die Produktivitätssteigerungen aufgrund einer verbesserten Gesundheit gegenüber (siehe hierzu beispielsweise Van Zon und Muysken (2001)).

Van Zon und Muysken (2005) beschreiben einen umgekehrt U-förmigen Zusammenhang zwischen der Beschäftigung im Gesundheitswesen und der Wachstumsrate des Konsums. Eine Ausweitung kurativer Maßnahmen bringt zunächst eine steigende Wachstumsrate mit sich, da sich die Gesundheit positiv auf die Zahl der Erwerbstätigen sowie auf die Produktivität auswirkt. Aufgrund der Annahme abnehmender Grenzerträge, die insbesondere durch die Annahme einer maximalen Gesundheit begründet ist, bringt eine weitere Steigerung der im Gesundheitssektor Beschäftigten keine weiteren Vorteile mit sich, da diese Arbeitskräfte den originären Produktionsprozessen entzogen werden. Somit steht bei einer Ausdehnung des Gesundheitssektors über das Optimum hinaus wieder weniger Einkommen für konsumtive Zwecke zur Verfügung.

\section{Konsumenten der Gesundheitsgüter}

An die Überlegung, wie die Produktionsfaktoren zwischen dem Gütersektor auf der einen und dem Sektor für Gesundheitsleistungen auf der anderen Seite alloziert werden sollen, schließt die Frage an, welche Bevölkerungsgruppe maßgeblich von diesen Leistungen profitiert.

Eindeutig positiv ist in diesem Zusammenhang zumindest aus wachstumstheoretischer Sicht die Bereitstellung präventiver Maßnahmen für Erwerbstätige zu bewerten, da in diesem Fall wiederum das effektive Arbeitsangebot positiv beeinflusst wird. Gleiches gilt für kurative Maßnahmen, die dem inaktiven Teil der Bevölkerung zugute kommen und die dazu beitragen, dass diese Personen wieder erwerbstätig werden können. Werden die Gesundheitsleistungen hingegen 
hauptsächlich von Rentnern nachgefragt, ergibt sich kein Effekt auf das effektive Arbeitsangebot, so dass diese Nachfrage nicht den Investitionen, sondern dem Konsum zuzurechnen ist. Damit folgen hieraus keinerlei Produktivitätseffekte in den volkswirtschaftlichen Produktionsprozessen.

Van Zon und Muysken (2001) ordnen eine Verlängerung der Lebenserwartung in diesen Kontext ein. Bei einer durchschnittlichen Lebenserwartung oberhalb des Renteneintrittsalters führt lebensverlängernder technischer Fortschritt in der Regel dazu, dass insbesondere der inaktive Teil der Bevölkerung hiervon profitiert. Somit steigt nicht nur die Zahl der inaktiven Personen, sondern es kommt zudem zu einer gesteigerten Nachfrage nach kurativen Gesundheitsleistungen. Definiert man nun das effektive Arbeitsangebot als das Verhältnis aus Aktiven zur Gesamtbevölkerung, so zeigt sich, dass technischer Fortschritt im Gesundheitswesen zumindest gesamtwirtschaftlich zu negativen Wachstumseffekten führen kann. ${ }^{35}$

Diese Argumentation gewinnt an Relevanz, wenn mit der gestiegenen Lebenserwartung zudem ein höherer Bedarf an Pflegeleistungen einhergeht, wie bei Van Zon und Muysken (2005) und Cremieux et al. (1999) angenommen. Tatsächlich ist der Bedarf an Pflegeleistungen insbesondere in Industrienationen durch die zunehmende Alterung der Bevölkerung sowie den technischen Fortschritt im letzten Jahrhundert stark gestiegen. Eine Reallokation der Ressourcen hin zu kurativen Maßnahmen, die insbesondere den Erwerbspersonen zugute kommen, würde langfristig zu einer Senkung der Pflegeausgaben führen und gleichzeitig die Zahl der Erwerbstätigen erhöhen. Diese neu verfügbare Arbeitskraft könnte entsprechend im Produktionssektor, im Gesundheitssektor oder im Bildungssektor eingesetzt werden. ${ }^{36}$ Letztlich ist eine derartige Umschichtung der finanziellen Ressourcen aber nur dann sinnvoll und wünschenswert, wenn die Finanzierung über den Abbau von Ineffizienzen im Gesundheitssystem oder in anderen öffentlichen Sektoren erfolgt, da sich ansonsten keine Pareto-Verbesserung einstellen kann.

Neben einem Anstieg der Lebenserwartung bedeutet auch eine Senkung der Kindersterblichkeit eine Zunahme des inaktiven Teils der Bevölkerung ${ }^{37}$, wenn

\footnotetext{
${ }^{35} \mathrm{Zu}$ den Einflüssen des medizinisch-technischen Fortschritts und einer hiermit verbundenen steigenden Lebenserwartung auf den durchschnittlichen Gesundheitszustand der Bevölkerung sowie die Gesundheitsausgaben siehe Ulrich (2001).

${ }^{36}$ Die von Van Zon und Muysken (2001) sowie (2005) gewählte Argumentation ist an den Humankapitalansatz zur Bewertung von Gesundheit angelehnt. $\mathrm{Zu}$ den Vorteilen, aber auch den Schwächen dieses Ansatzes siehe beispielsweise Breyer et al. (2005), S. 43f.

${ }^{37}$ Diese Annahme basiert auf der Überlegung, dass Kinder dem Arbeitsmarkt zunächst nicht zur Verfügung stehen und so dem inaktiven Teil zugerechnet werden müssen.
} 
Eltern diese nicht in ihrer Familienplanung berücksichtigen. Insbesondere in ärmeren Ländern ist ein Rückgang der Kindersterblichkeit jedoch nicht ad hoc zu beobachten, so dass damit zu rechnen ist, dass sich eine geringere Sterberate positiv auf die Kinderzahl auswirkt und so das Durchschnittsalter der Bevölkerung senkt. In einem solchen Fall verringern sich ebenfalls die Erwerbsquote und damit auch das Pro-Kopf-Einkommen. In der Folge stehen letztlich nicht nur weniger Ressourcen für Gesundheitsleistungen zur Verfügung, sondern auch für die Ernährung sowie die Ausbildung von Kindern, weshalb das erwartete Lebenseinkommen trotz einer Verbesserung der Gesundheit sogar sinkt (vgl. hierzu Howitt (2005)).

Die Bedeutung des Konsums von Gesundheitsleistungen lässt sich wie folgt zusammenfassen: Eine gesundheitsbedingte zunehmende Alterung der Bevölkerung, die sich bereits im Rentenalter befindet, bringt einen höheren Bedarf an Konsum- und Gesundheitsgütern mit sich, ohne dass diesem Bedarf eine gesteigerte Produktion gegenübersteht. Damit sinkt das pro Kopf verfügbare Einkommen. ${ }^{38}$ Eine Verbesserung der Gesundheit der Erwerbstätigen ist hingegen für das Wachstum förderlich, da diese für die Güterproduktion, für die Erstellung der Gesundheitsleistungen sowie für die Akkumulation von Bildungskapital verantwortlich sind.

\section{Direkte versus indirekte Produktivitätseffekte}

Eine gute Gesundheit kann nun auf der einen Seite dazu führen, dass die Produktivität der Erwerbstätigen steigt, was als direkter Produktivitätseffekt bezeichnet wird. Auf der anderen Seite steigt zudem die Produktionseffizienz bei der Akkumulation von Bildungskapital, woraus sich letztlich ein indirekter Produktivitätseffekt ableitet. Aufgrund der theoretischen Begrenzung des Gesundheitskapitalbestandes sind auch die Produktivitätseffekte, die sich allein aus einer verbesserten Gesundheit ergeben können, nach oben begrenzt. Zudem ließe sich ein theoretisch perfekter Gesundheitszustand aufgrund der Annahme abnehmender Skalenerträge sowie aufgrund des Vorliegens von Abschreibungen

${ }^{38}$ Im Jahr 1950 waren 14,6\% der Bevölkerung Deutschlands 60 Jahre alt oder älter, insgesamt ca. 1 \% war sogar über 79. 30,4\% waren hingegen jünger als 20 Jahre. 2001 betrug der Anteil der über 60-jährigen bereits $24,1 \%$, während der Anteil der unter 20-jährigen auf 20,9\% gesunken ist. Prognosen zufolge wird die Zahl der Jugendlichen bis zum Jahr 2050 auf ca. 16,1 \% absinken, wăhrend bereits $36,7 \%$ zu den uber 60 -jährigen zu zăhlen sein werden, und sogar 12,1 \% werden über 80 Jahre alt sein. Der Anteil der Erwerbstătigen (20 bis 59 Jahre) wird demnach nur noch 47,2 \% betragen (vgl. Statistisches Bundesamt (2003), S. 31). In Anbetracht der Tatsache, dass die Gesundheitsausgaben mit dem Alter immer weiter ansteigen (vgl. Kruse et al. (2003), S. 34-43), werden sich die negativen Wachstumseffekte des gesteigerten Bedarfs an Gesundheits- und Pflegeleistungen in der Zukunft noch verstärken. 
nur unter prohibitiv hohen Kosten erreichen. Aus diesen Überlegungen lässt sich schlussfolgern, dass Gesundheit per se kein Wachstumsmotor sein kann.

Anders verhält es sich mit dem Einfluss der Gesundheit auf die Akkumulation von Bildungskapital. Eine gute Gesundheit erhöht die Konzentrations- und Leistungsfähigkeit, wodurch die Generierung neuen Wissens erleichtert wird. Zudem senkt eine verbesserte Gesundheit die Gegenwartspräferenz, da der Planungshorizont der Individuen verlängert wird und Investitionen in das Bildungskapital lohnender werden, so dass auch die Qualität der angebotenen Arbeitskraft zunimmt. Ein Anstieg im durchschnittlichen Gesundheitskapital bringt folglich positive Bildungseffekte mit sich, woraus sich beispielsweise im Ansatz von Van Zon und Muysken (2001) ein positiver Einfluss auf das Wirtschaftswachstum ableitet.

Letztlich kann dieser indirekte Produktivitätseffekt sogar dazu führen, dass es zu Aufholprozessen eines armen Landes und damit zu hohen Wachstumsraten kommt. Howitt (2005) nimmt in seinem Wachstumsmodell zunächst an, dass Einkommensunterschiede zwischen reichen und armen Ländern dauerhaft bestehen bleiben, da technischer Fortschritt innerhalb eines Landes nicht nur von der eigenen Innovationstätigkeit abhängt, sondern da sich zudem Spillover-Effekte ergeben, indem technische Innovationen aus anderen Ländern ebenfalls nutzbar und imitierbar sind und für weitere Neuerungen herangezogen werden können. Vorangegangene Erfindungen werden aufgrund technischer Neuerungen hingegen obsolet. Für ärmere Länder gilt nun, dass diese aufgrund fehlender Bildung und aufgrund regionaler Disparitäten nicht in vollem Umfang von den Wissenspillovern profitieren, weshalb es auch nicht zu Konvergenz kommen kann.

Eine gezielte Verbesserung des durchschnittlichen Gesundheitszustandes in einkommensschwachen Ländern kann nun einen Aufholprozess initiieren. Durch eine gesteigerte Produktivität können die bereits vorhandenen Produktionsfaktoren wie physisches Kapital, Allgemeinbildung oder technisches Know-how besser genutzt werden und so zu einem höheren Outputniveau führen. Das derart erworbene zusätzliche Einkommen kann für Investitionen genutzt werden und so zu Innovationen oder Imitationen führen, wodurch die gleichgewichtige Wachstumsrate positiv beeinflusst wird. Für die reicheren Länder würde sich diesen Annahmen zufolge zwar kein langfristiger Effekt auf die gleichgewichtige Wachstumsrate ergeben, wohl aber auf das Pro-Kopf-Einkommen, das durch eine erhöhte Produktivität ebenfalls gesteigert werden kann. 
Positive externe Effekte

Analog zum Bildungskapital kann auch die Akkumulation von Gesundheitskapital mit positiven externen Effekten verbunden sein, wenn Unternehmen die Gesundheit als unveränderlichen Faktor betrachten (vgl. Johansson und Löfgren (1995)). Aufgrund der Exogenität werden Produktivitätseffekte, die aus einer verbesserten Gesundheit resultieren, in den Lohnzahlungen nicht berücksichtigt. Damit bieten die Erwerbstätigen ihr Gesundheitskapital zusätzlich zur eigentlichen Arbeitskraft kostenlos an. Eine angemessene Berücksichtigung der Gesundheit als Produktionsfaktor würde entsprechend positive Anreize zur Gesundheitskapitalakkumulation setzen.

Letztlich besteht für das einzelne Unternehmen jedoch keine Motivation, in die Gesundheit der Beschäftigten zu investieren. Die Argumentation erfolgt hier analog zur Finanzierung von allgemeiner Ausbildung (vgl. Becker (1993)): Würden sich Unternehmen bemühen, in die Gesundheit ihrer Beschäftigten zu investieren, so würde sich deren allgemein nutzbares Humankapital erhöhen. Konkurrierende Unternehmen würden diese Arbeitskräfte abwerben, indem sie höhere Löhne anböten, weil der realisierbaren Produktivitätssteigerung keine entsprechenden Kosten gegenüberstünden. Obwohl eine gute Gesundheit der Beschäftigten daher für jedes Unternehmen absolut wünschenswert ist, kommt es zu Unterinvestitionen, da stets die Befürchtung eines Arbeitsplatzwechsels besteht. $^{39}$

Im Rahmen der vorangegangenen theoretischen Überlegungen zeigt sich, dass sich aus der Gesundheit per se zwar kein endogenes Wachstum entwickeln kann, eine gute Gesundheit der Bevölkerung jedoch zu einer optimalen Auslastung des Produktionsfaktors Humankapital führt. Darluber hinaus kann ein hoher Gesundheitskapitalstock vermehrte Bildungsinvestitionen mit sich bringen, woraus sich dauerhaft höhere Wachstumsraten ergeben können. Schlechte Gesundheit wirkt sich hingegen negativ auf das Wirtschaftswachstum aus, da ein Teil der Produktionsfaktoren im Gesundheitssektor gebunden wird und so anderen Produktionszwecken nicht weiter zur Verfügung steht. Dieses Argument wird insbesondere

${ }^{39}$ Dieses Argument lässt sich abschwächen, wenn die Arbeitgeber an der Finanzierung der Krankenversicherung beteiligt sind, da sie in diesem Fall ein Interesse an geringen Versicherungsprämien bzw. Beitragssätzen haben. Investitionen in die Gesundheit ihrer Beschäftigten werden in diesem Fall theoretisch solange durchgefuhrt, wie die entstehenden Kosten durch einen Rückgang der Zahlungen an die Versicherung kompensiert werden. In einem gesetzlichen Krankenversicherungssystem ist dieser Anreiz insbesondere für kleine Unternehmen jedoch verschwindend gering, da diese die Gesundheitsausgaben der Gesamtbevőlkerung nur marginal beeinflussen. 
dann bestärkt, wenn die Gesundheitsleistungen insbesondere dem alten, inaktiven Teil der Bevölkerung zugute kommen.

\subsubsection{Empirische Befunde}

Zahlreiche Studien haben bereits den positiven Einfluss der Bildung auf das Wirtschaftswachstum empirisch bestätigt (vgl. Dinopoulos und Thompson (1996), Goetz und Hu (1996), Bernanke und Gürkaynak (2001), Barro (2001)), ohne jedoch den positiven Einfluss der Gesundheit auf die Humankapitalakkumulation zu berücksichtigen. Mankiw et al. (1992) berücksichtigen die Gesundheit zwar, indem sie Humankapital allgemein in ihrer theoretischen Modellierung verwenden, im empirischen Teil der Arbeit ist Gesundheit jedoch nicht enthalten.

Fogel (1994) zeigt erstmals die besondere Bedeutung der Gesundheit für das Wirtschaftswachstum auf. Gemäß dieser Studie lassen sich 30 Prozent des britischen Wirtschaftswachstums durch Verbesserungen in der Ernährung erklären, wobei ein Zeitraum ab Ende des 18. Jahrhunderts berücksichtigt wird. Darüber hinaus lassen sich 20 Prozent des Wachstums durch einen Anstieg der Erwerbsquote sowie längere Arbeitszeiten erklären, so dass der Gesamteffekt einer Verbesserung der Ernährung bei etwa 50 Prozent liegt.

Nun sind derartige Daten nur für wenige Länder verfügbar und insbesondere nicht für Entwicklungsländer. Daher verwendet ein Großteil der empirischen Studien die Lebenserwartung als Gesundheitsindikator (zu einem umfassenden Überblick siehe Bloom et al. (2004)). Knowles und Owen (1995) und (1997) wenden beispielsweise den neoklassischen Ansatz von Mankiw et al. (1992) an, wobei Bildung und Gesundheit nun explizit berücksichtigt werden.

Unter Verwendung der Ausbildungsdauer als Indikator für das Bildungskapital sowie der Lebenserwartung als Maß für die Gesundheit kommen Knowles und Owen (1995) in ihrer Studie zu dem Ergebnis, dass die Gesundheit einen auf dem 1\%-Level signifikanten positiven Einfluss auf die Wachstumsrate des ProKopf-Einkommens ausübt. Der Einfluss der Bildung, der in den oben genannten Studien als hoch signifikant ausgewiesen wurde, ist in dieser Studie als nicht signifikant bewertet, solange Gesundheitskapital als zusätzliche erklärende Variable in dem Regressionsmodell enthalten ist.

Der zweiten Studie von Knowles und Owen (1997) liegt die Annahme zugrunde, dass das Humankapital nicht mehr als eigenständiger Produktionsfaktor zu sehen ist, sondern sich arbeitsvermehrend auswirkt. Diese Annahme impliziert, dass sowohl das Bildungs- als auch das Gesundheitskapital nicht notwendigerweise zu einem Steady State konvergieren, diese Faktoren also nicht zwingend 
Wachstumsgrenzen unterliegen (vgl. Knowles und Owen (1997), S. 315). Hieraus folgt, dass die Wachstumsraten auch im Steady State nicht miteinander übereinstimmen müssen. Unter Wahl identischer Indikatoren für die Regressoren unterscheidet sich das Ergebnis dieser Studie im Wesentlichen nicht von der Studie aus dem Jahr 1995. Die Gesundheit hat den postulierten signifikant positiven Einfluss auf die Wachstumsrate des Volkseinkommens, während der Einfluss der Bildung zwar positiv, jedoch erneut nicht signifikant ist.

Barro und Sala-i-Martin (2003) verwenden den Kehrwert der Lebenserwartung im Alter von einem Jahr. Dieser beschreibt die Mortalitätsrate unter der Annahme, dass Mortalität altersunabhängig ist. Der geschätzte Koeffizient ist negativ und hoch signifikant, so dass sich ein Anstieg in der Lebenserwartung positiv auf das Wirtschaftswachstum auswirkt.

Gyimah-Brempong und Wilson (2004) verwenden ebenfalls die Lebenserwartung als Indikator für den Gesundheitszustand, berücksichtigen darüber hinaus aber auch den Anteil der Gesundheitsausgaben am BIP als Indikator für die Investitionen in die Gesundheit. ${ }^{40}$ Der Wachstumseffekt der Lebenserwartung ist hier ebenfalls signifikant positiv, wenn auch mit abnehmender Rate, und auch für die Investitionen in die Gesundheit sowie die Schulbildung kann ein positiver Einfluss festgestellt werden. ${ }^{41}$

Die Verwendung der Lebenserwartung als Gesundheitsindikator ist jedoch nicht unproblematisch. Mayer-Foulkes (2005) zeigt, dass in einem Teil der Länder die Lebenserwartung niedrig ist, während insbesondere die reicheren Länder über eine relativ hohe Lebenserwartung verfügen, so dass hier letztlich zwei verschiedene Ländergruppen existieren. Implizit wird eine Verbesserung des Gesundheitszustandes angenommen, je höher die Lebenserwartung ist. Es gibt jedoch auch Gründe, die gegen einen solchen Zusammenhang sprechen.

Erstens kann für Industrienationen angenommen werden, dass Krankheiten, die ehemals tödlich verliefen, durch den medizinisch-technischen Forschritt geheilt werden können. Sinkt hierdurch allein die Überlebensschwelle, indem die zusätzliche Lebensdauer mit dauerhafter Krankheit verbunden ist, dann führt ein Anstieg der Lebenserwartung zu einem Absinken des durchschnittlichen Gesundheitszustandes, so dass sich der postulierte positive Zusammenhang nicht

\footnotetext{
${ }^{40}$ Private Ausgaben werden allerdings nicht berücksichtigt, so dass der Zusammenhang zwischen den Gesundheitsausgaben und dem Wirtschaftswachstum eher unterschätzt wird.

${ }^{41}$ Arora (2001) gibt zudem zu bedenken, dass die Zahl der Schuljahre ausschließlich die Schulbildung wiedergibt, nicht aber die Berufserfahrung und damit hiermit verbundene Learning by doing. Zudem werden Unterschiede in der Qualität der Schulen ebenfalls nicht abgebildet.
} 
mehr einstellt. Somit ist anzunehmen, dass keine eindeutige Korrelation zwischen Morbidität und Mortalität besteht (vgl. Rivera und Currais (1999), S. 761). Eine Aussage über einen über alle Individuen aggregierten durchschnittlichen Gesundheitszustand ließe sich somit bestenfalls dann treffen, wenn neben der Mortalität beispielsweise auch der Grad der medizinischen Versorgung, Ernährungs- und andere Lebensgewohnheiten sowie Umweltseinflüsse berücksichtigt würden (vgl. Bhargava et al. (2001), S. 423). ${ }^{42}$ Darüber hinaus ist ein Anstieg der Lebenserwartung insbesondere dann negativ zu bewerten, wenn hierdurch lediglich die Zahl der inaktiven Bevölkerung steigt, so dass keine weiteren Produktivitätseffekte realisiert werden können.

Problematisch ist die Verwendung der Lebenserwartung auch dann, wenn sich ein Anstieg insbesondere aus einer Senkung der Kindersterblichkeit ergibt, da es in diesem Fall zu Bevölkerungswachstum kommt, ohne dass die Zahl der Arbeitskräfte adäquat steigt. Folglich sinkt zunächst das Pro-Kopf-Einkommen, so dass sich ein positiver Effekt erst dann einstellen kann, wenn auch die Geburtenrate entsprechend abnimmt (vgl. Kalemli-Ozcan (2002)).

Um diese Probleme zu vermeiden, verwenden einige Studien (Bhargava et al. (2001), Arora (2001), Jamison et al. (2005) und Weil (2005)) das Maß der "adult survival rate", das die Wahrscheinlichkeit, bei einem bereits erreichten Alter von 15 Jahren 60 Jahre alt zu werden, angibt. Diese Studien kommen allesamt zu dem Resultat, dass ein Anstieg dieser Wahrscheinlichkeit Wirtschaftswachstum erklärt und somit eine Verbesserung der Gesundheit zu einer Verringerung der Einkommensunterschiede führen würde.

Nun ist auch die Überlebensrate kein eindeutiger Indikator für Produktivitätszuwächse. Nach Ansicht von Jamison et al. (2005) resultiert technischer Fortschritt insbesondere aus Bildung und weniger aus einem Anstieg der Lebenserwartung, da letzterer eher das Einkommensniveau per se beeinflusst. Zudem liefert ein langes Leben maßgebliche Anreize zu mehr Bildungsinvestitionen, weshalb hier die Gefahr besteht, dass die Effekte aus Gesundheit und Bildung nicht eindeutig getrennt werden können.

Rivera und Currais (1999), (2000) und (2004) verwenden aus diesen Gründen jeweils die öffentlichen Konsumausgaben für Gesundheit als Indikator für $\mathrm{Ge}$ sundheitsinvestitionen, auch wenn diese nicht zwangsläufig Aufschluss über die

\footnotetext{
${ }^{42}$ Hierzu ist anzumerken, dass die Morbidität in dem untersuchten Kontext einen besseren Indikator für den Gesundheitszustand darstellt, da die Krankheitsanfälligkeit der Individuen ihre Produktivität und damit das Wachstum stärker beeinflusst als die Mortalität, die zumindest in entwickelten Ländern erst im Rentenalter eine Rolle spielt.
} 
Effektivität geben, mit der Gesundheitsausgaben in Gesundheitskapital umgewandelt werden können. Darüber hinaus werden Investitionen in die Infrastruktur des Gesundheitsmarktes berücksichtigt, um hieraus mögliche Standortvorteile abzuleiten. Die Ergebnisse sind trotz Verwendung unterschiedlicher Daten eindeutig, denn mit den Gesundheitsausgaben verbundene Konsumeffekte medizinischer Leistungen wirken sich jeweils signifikant positiv aus, während der Einfluss der Infrastruktur nicht signifikant ist. ${ }^{43}$

\subsection{Zwischenergebnis}

Abbildung 5 stellt die Zusammenhänge zwischen der Gesundheit und endogenem Wachstum noch einmal überblicksartig dar. Anhand der Pfeilstruktur werden Interdependenzen zwischen den einzelnen Determinanten des Wachstums sichtbar. Diese Grafik zeigt, dass Gesundheit und Bildung das Wirtschaftswachstum über die Produktivität gemeinsam determinieren und einander zudem bedingen. Des Weiteren bestehen Wechselwirkungen zwischen diesen Wachstumsfaktoren und dem Konsum von Gesundheitsleistungen sowie dem erreichbaren Technologieniveau.

Der entscheidende Einfluss von Gesundheit und Bildung ist in den hieraus resultierenden Produktivitätseffekten zu sehen. Diese führen zum einen dazu, dass schneller und möglichst effizient produziert werden kann, zum anderen ist ein höherer Grad an Aus- und Weiterbildung erreichbar, wodurch wiederum die gesamte Humankapitalakkumulation gesteigert werden kann. Als Konsequenz hieraus kann sich eine gesteigerte Innovationstätigkeit ergeben. Ferner ergeben sich zusätzlich positive Effekte im Hinblick auf die Lernfähigkeit von Kindern, wodurch Voraussetzungen für das zukünftige Bildungskapital sowie die daraus resultierende Produktivität geschaffen werden.

Die indirekten Effekte der Gesundheit sind in Abbildung 5 durch den Einfluss auf den Gesundheitsmarkt als eigenständigen Wachstumsmarkt gekennzeichnet. Eine zusätzliche Nachfrage nach Gesundheitsleistungen aufgrund von gesteigertem Investitionsverhalten zieht eine Ausweitung des Angebots nach sich. Hieraus folgt zum einen positives Wachstum aufgrund der Wertschöpfung des Gesundheitssektors, zum anderen werden Einkommens- und Produktivitätsverluste, die aufgrund von Krankheiten entstehen, gesenkt sowie die Zahl der verfügbaren Arbeitskräfte erhöht. Diesen positiven Konsequenzen einer umfassenden Gesundheitsversorgung steht die Tatsache entgegen, dass die im Gesundheitssektor gebundene Arbeitskraft sowie deren Humankapitalbestand nicht al-

\footnotetext{
${ }^{43}$ Rivera und Currais (2004) begründen diesen fehlenden Zusammenhang mit einer zeitverzögerten Wirkung, die nicht durch die Daten abgebildet wird.
} 
ternativ in den Produktionsprozess eingesetzt sowie für die Akkumulation von physischem sowie Humankapital verwendet werden können (vgl. Rivera und Currais (1999), S. 761). Das gleiche Argument ließe sich jedoch auch für das gebundene Kapital anführen, das ebenfalls alternativen Produktionsverwendungszwecken entzogen wird. ${ }^{44}$

Abbildung 5: Schematische Darstellung der Zusammenhänge zwischen Gesundheit, Bildung und Wirtschaftswachstum

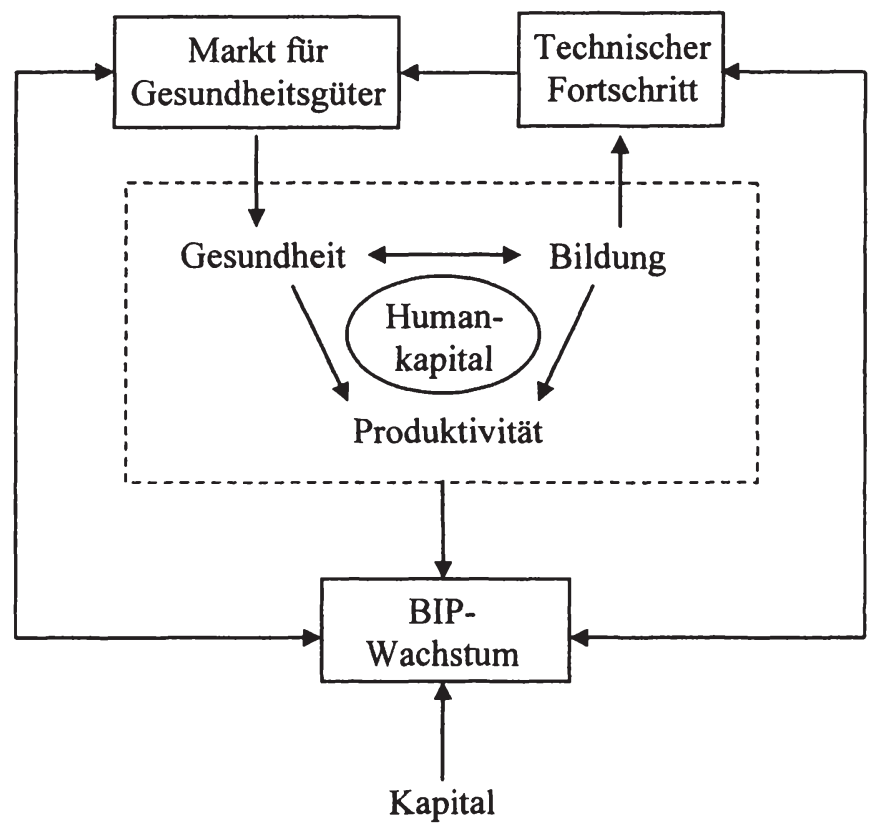

Quelle: Eigene Darstellung.

Für die Bildung gilt, dass diese sich nicht nur produktivitätssteigernd auswirkt, sondern auch eine wesentliche Voraussetzung für Forschung und Entwicklung ist. Hierüber wird zum einen direkt Wirtschaftswachstum generiert, zum anderen führen Innovationen zu besseren Behandlungsmethoden von Krankheiten. Folglich beeinflusst ein höherer Bildungskapitalbestand die Gesundheit von In-

\footnotetext{
${ }^{44}$ Zudem ist es durch die Beschaffenheit des Gesundheitsmarktes möglich, dass aufgrund der vorherrschenden unvollständigen Information im Gesundheitswesen das Angebot seine Nachfrage bestimmt. Sobald die Allokation medizinischer Leistungen ineffizient ist, wirkt die gesteigerte Nachfrage nicht mehr wachstumsfördernd, sondern sogar wachstumshemmend (zu dieser These der angebotsinduzierten Nachfrage siehe Breyer et al. (2005)).
} 
dividuen nicht nur über ein verbessertes gesundheitsrelevantes Verhalten, sondern auch über die gesteigerte Innovationstätigkeit und den hiermit verbundenen technischen Fortschritt im Gesundheitswesen. 


\section{Investitionen in die Gesundheit - Das Grossman-Modell}

\subsection{Die Nachfrage nach Gesundheit}

Im vorangegangenen Kapitel wurden zahlreiche Möglichkeiten aufgezeigt, über die der verfügbare Gesundheitskapitalstock das Wirtschaftswachstum beeinflusst. Es ergeben sich zum einen direkte Effekte auf das effektive Arbeitsangebot, indem die für Arbeit verfügbare Zeit ausgeweitet und die Produktivität erhöht wird. Zum anderen existieren zahlreiche indirekte Effekte, indem die Quantität sowie die Qualität der Schulbildung positiv beeinflusst werden. Gleichzeitig wurde angenommen, dass der Gesundheitskapitalstock einer Gesellschaft nicht exogen gegeben ist, sondern dass vielmehr Investitionen in die Gesundheit zu einer Verbesserung derselben führen können, während altersoder verhaltensbedingte Abschreibungen das verfügbare Gesundheitskapital mindern.

Diese Idee des gesundheitsrelevanten Verhaltens geht auf die Arbeiten von Mushkin (1962) sowie insbesondere Grossman (1970) und (1972) zurück. Ein Blick in die aktuelle gesundheitsökonomische Literatur zeigt, dass das Grossman-Modell auch 35 Jahre nach seinem Erscheinen noch Grundlage vieler weiterführender Arbeiten ist (vgl. z. B. den 23. Jahrgang des Journal of Health Economics ${ }^{45}$ ). Auch wenn dieses Modell mittlerweile zur gesundheitsökonomischen Basisliteratur gehört, sollen die Grundannahmen, die wesentlichen Ergebnisse sowie Kritikpunkte nachfolgend kurz erläutert werden, da sich auch Überlegungen aus den nachfolgenden Kapiteln, insbesondere jedoch die weiterführende Modellanalyse des fünften Kapitels, auf das Grossman-Modell beziehen.

Grossman zufolge entspricht die Nachfrage nach Gesundheitsleistungen einer abgeleiteten Nachfrage, die einem Individuum einzig dazu dient, seinen gewünschten Gesundheitskapitalstock zu erreichen. Dieses Ergebnis ergibt sich aus der Überlegung, dass jedes Individuum über einen anfänglichen Gesundheitskapitalstock verfügt, der über die Zeit Abschreibungen unterliegt, aber auch durch Investitionen vergrößert werden kann. Somit kann jede Person seinen Gesundheitskapitalbestand zu jedem Zeitpunkt selbst bestimmen und somit auch die Lebenslänge selbst beeinflussen. Darüber hinaus weist Grossman der Gesundheit einen Konsum- und einen Investitionsgutcharakter zu, denn zum einen dient eine gute Gesundheit der direkten Nutzenstiftung, zum anderen determi-

\footnotetext{
${ }^{45}$ Diese Ausgabe enthält Beiträge eines gesundheitsökonomischen Symposiums mit dem Titel "The Demand for Health, 30 Years Later: What Do We Know? What Do We Need to Know?", das im Januar 2003 unter Federführung der ,American Economic Association' (AEA) sowie der ,International Health Economics Association' (iHEA) stattfand.
} 
niert sie die Leistungsfähigkeit am Arbeitsmarkt, woraus sich schließlich das Erwerbseinkommen ableitet. Grossman behandelt diese Effekte der Gesundheit getrennt, indem zunächst ein reines Investitionsmodell behandelt und anschließend der Nutzenaspekt in Form eines Konsummodells herausgearbeitet wird. Da Gesundheit im Rahmen dieser Arbeit jedoch hauptsächlich als Investitionsgut verstanden wird, wird nachfolgend auf eine Darstellung des Konsummodells verzichtet. $^{46}$

Unter der Annahme, dass Investitionen in die Gesundheit durch den Konsum medizinischer Leistungen sowie Zeitaufwand bestimmt werden, ergibt sich für das Individuum ein Optimierungsproblem in der Zeitallokation: Je mehr Zeit für Investitionen in die Gesundheit genutzt wird, desto geringer ist zwar die krank verbrachte Zeit, desto weniger Zeit steht jedoch zunächst auch für Arbeit und damit für die Erzielung von Erwerbseinkommen zur Verfügung. ${ }^{47}$

\subsection{Der optimale Gesundheitskapitalstock}

Analog zu übrigen Investitionsentscheidungen müssen sich die Gegenwartswerte von Grenzerträgen und Grenzkosten der Investition in die Gesundheit im Optimum zu jedem Zeitpunkt entsprechen. ${ }^{48}$ Beschreibt der Grenzertrag des Gesundheitskapitalstocks in diesem Kontext einen Anstieg der Anzahl gesund verbrachter Tage aufgrund eines marginalen Anstiegs des Gesundheitskapitalbestandes, so ist die optimale Investitionshöhe dann erreicht, wenn das auf den Gegenwartswert diskontierte Wertgrenzprodukt der Investitionen gleich den abgezinsten Grenzkosten ist. Dementsprechend muss die Rendite des Gesundheitskapitalstocks in Form des Grenzertrags im Optimum zu jedem beliebigen Zeitpunkt genau den Nutzungskosten des Gesundheitskapitals entsprechen, die durch den Zinssatz als Preis des Kapitals sowie die Abschreibungsrate definiert sind. ${ }^{49}$

In Abbildung 6 wird der optimale Gesundheitskapitalstock für jeden beliebigen Zeitpunkt dargestellt. Die Abszisse enthält den jeweiligen Gesundheitskapitalbestand $H_{i}$, auf der Ordinate sind sowohl der Grenzertrag als auch die Grenzkos-

\footnotetext{
${ }^{46}$ Freizeit wird selbst im reinen Investitionsmodell berucksichtigt, solange diese nicht zur Erzielung von Erwerbseinkommen verwendet wird (vgl. Erbsland et al. (1998), S. 15).

${ }^{47}$ Zur Theorie der Zeitallokation siehe z. B. Becker (1965) und Ben-Porath (1967).

${ }^{48}$ Dieses Ergebnis resultiert letztlich aus der Annahme einer additiv-separablen Nutzenfunktion.

49 Die monetäre Bewertung der hinzugewonnenen Zeit erfolgt im Modell einerseits über die Höhe des zusătzlich erzielbaren Einkommens, andererseits über ein geldwertes Äquivalent des Nutzenzuwachses (vgl. Grossman (1972), S. 228f.).
} 
ten abgetragen. Die Nachfragekurve $M E C^{50}$, die den Grenzertrag der Investition beschreibt, gibt Aufschluss über die Beziehung zwischen diesem Ertrag und dem Gesundheitskapitalstock, während die Angebotskurve $S$ die Kapitalkosten beschreibt. Sowohl der Zinssatz als auch die Abschreibungsrate sind vom Gesundheitszustand unabhängig, weshalb die Angebotskurve waagerecht verläuft. Folglich ergeben sich für jeden Gesundheitskapitalbestand $H_{i}$ Kosten in Höhe von $S$. Für die Nachfragekurve ergibt sich unter Annahme eines sinkenden Grenzprodukts des Kapitals ein fallender Verlauf mit steigendem Bestand an Gesundheitskapital. Im Schnittpunkt dieser beiden Kurven entsprechen die Grenzerträge der Gesundheit gerade den Grenzkosten, so dass hier der gleichgewichtige Gesundheitskapitalstock $H_{1}{ }^{*}$ erreicht wird.

Dieser optimale Zustand verändert sich nur dann, wenn sich entweder die Grenzerträge oder aber die Grenzkosten ändern. Die Grenzkosten steigen beispielsweise, wenn die Abschreibungsrate auf die Gesundheit nicht mehr konstant ist, sondern mit dem Alter ansteigt. ${ }^{51}$ Je höher c. p. die Abschreibungsrate ist, desto weiter verschiebt sich die Grenzkostenkurve nach oben $\left(S^{\prime}\right)$, während der Verlauf der Grenzertragskurve hiervon unbeeinflusst bleibt. Somit verschiebt sich auch der Schnittpunkt zwischen diesen beiden Kurven immer weiter nach links. Im Punkt $H_{\min }$ ist schließlich der minimale Gesundheitskapitalstock erreicht, durch den der Todeszeitpunkt bestimmt wird.

Aus der Annahme der altersabhängigen Abschreibungsrate folgt ein durchschnittlicher Gesundheitszustand, der sich mit zunehmendem Alter verschlechtert, da es immer mehr Investitionen bedarf, um dieser Entwicklung entgegenzuwirken. Sind die Grenzerträge der Investitionen irgendwann zu gering, um die hohen Abschreibungen zu kompensieren, wird nicht weiter in die Gesundheit investiert. In diesem Fall wird nach dem Modell Grossmans der Todeszeitpunkt gewählt. Damit bestimmen die einzelnen Individuen nicht nur ihre eigene Gesundheit, sondern sie wählen auch die Länge ihres Lebens.

Eine weitere Beeinflussung des optimalen Gesundheitskapitalstocks erfolgt z. B. über den Lohnsatz, da dieser den Grenzertrag der Gesundheit bestimmt. Je höher der Lohnsatz ist, desto wertvoller ist gleichzeitig die gesund verbrachte Zeit, da über jede zusätzlich verfügbare Zeiteinheit weiteres Einkommen erzielt werden kann. Dementsprechend verschieben sich die Grenzertragskurve sowie der op-

${ }^{50} M E C=$ marginal efficiency of health capital.

${ }^{51}$ Diese Annahme wird ebenfalls aus Vereinfachungsgründen getroffen, da Schwankungen in der Abschreibungsrate ausgeblendet werden sollen. Somit werden auch die erhöhte Krankheitsgefahr im Kindesalter sowie Stressfaktoren an dieser Stelle nicht berücksichtigt. 
timale Gesundheitskapitalbestand nach rechts, so dass der neue optimale Gesundheitskapitalstock $\mathrm{H}_{2}{ }^{*}$ erreicht wird.

Abbildung 6: Der optimale Gesundheitskapitalstock

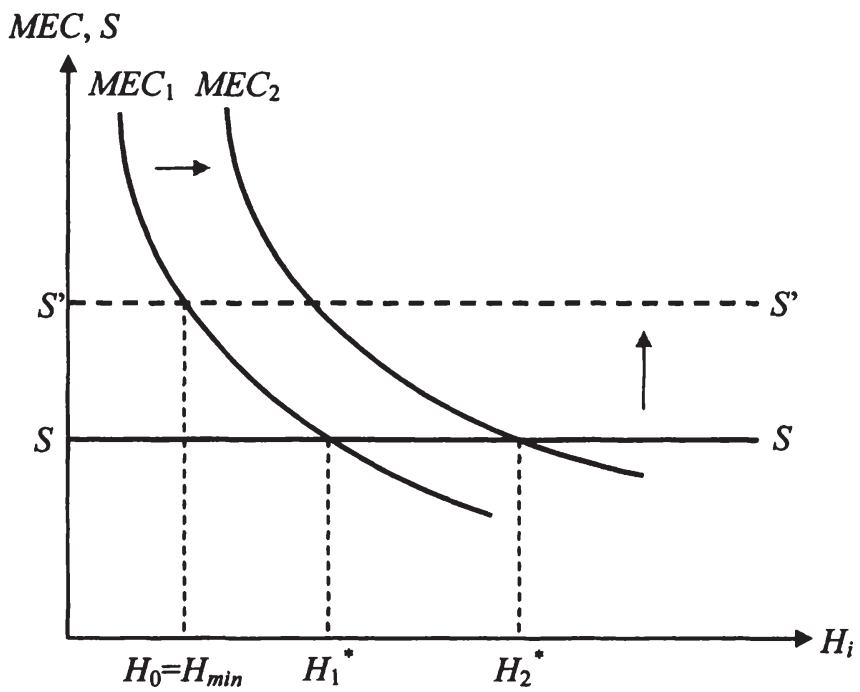

Quelle: Vgl. Grossman (1972), S. 237.

\subsection{Modellkritik und Erweiterungen des Grossman-Modells}

Grossman zeigt, dass jedes Individuum über einen optimalen Gesundheitskapitalbestand verfügt und die Nachfrage nach medizinischen Leistungen von diesem Optimum abhängig ist. Die Verantwortung für die Gesundheit liegt somit bei jedem selbst. Diese Modellierung ist zwar plausibel in dem Sinne, dass Individuen über ihren gesundheitsorientierten Zeitaufwand sowie den Konsum von Gesundheitsleistungen dazu beitragen können, einen gewünschten Gesundheitszustand zu erreichen.

Gleichzeitig existieren jedoch zahlreiche Kritikpunkte, welche nachfolgend kurz skizziert und erläutert werden:

Grossman nimmt in seinem Modell vollkommene Information hinsichtlich des Todeszeitpunkts an, Unsicherheit hierüber wird demnach nicht in die Analyse einbezogen. Damit wird auch eine geringere Nachfrage nach medizinischen Leistungen vorhersagt, als dies tatsächlich der Fall ist, da sämtliche Investitio- 
nen in die Gesundheit, die aufgrund von Unsicherheit getätigt werden, nicht berücksichtigt sind (vgl. Grossman (1972), S. 247). ${ }^{52}$ Nimmt man stattdessen an, dass Krankheit ein zufälliges Ereignis ist, so dienen Investitionen in die Gesundheit nicht nur einer der Verbesserung der Gesundheit selbst, sondern vor allem auch der Minimierung der Erkrankungswahrscheinlichkeit. Insofern werden Investitionen in die Gesundheit auch zu Präventionszwecken getätigt, wodurch die Nachfrage nach Gesundheitsleistungen gegenüber der Situation unter Sicherheit ansteigt (vgl. hierzu das Lebenszyklusmodell von Cropper (1977)). Auch besteht im Grossman-Modell keinerlei Unsicherheit über die Qualität der medizinischen Behandlung, so dass Informationsasymmetrien sowie PrincipalAgent-Probleme ausgeblendet werden. Dardanoni und Wagstaff (1990) zeigen in diesem Zusammenhang, dass die Nachfrage nach Gesundheitsleistungen sinkt, sobald die Unsicherheit über die Qualität gemindert wird oder Effizienzsteigerungen im Gesundheitssektor erwartet werden.

Unsicherheit über das zukünftige Erwerbseinkommen, welches im GrossmanModell exogen gegeben ist, führt darüber hinaus zu Ersparnisbildung aus Vorsichtsmotiven, weswegen wiederum weniger Ressourcen für Investitionen in die Gesundheit aufgewendet werden (vgl. Selden (1993)). Einem solchen Verhalten könnte beispielsweise durch die Einführung einer Krankenversicherung entgegengewirkt werden (vgl. Liljas (1998)).

Weiterhin kann es sein, dass es zu einer Diskrepanz zwischen der gewünschten und der tatsächlichen Inanspruchnahme medizinischer Leistungen kommt, wovon im Modell ebenfalls abstrahiert wird. Sämtlicher Konsum geht folglich direkt vom einzelnen Individuum bzw. Patienten aus, während angebotsinduzierte Nachfrage hier keine Rolle spielt (vgl. Wagstaff (1986), S. 210). Aufgrund von Informationsasymmetrien über die Notwendigkeit bestimmter Behandlungen einerseits sowie die erforderliche Menge andererseits wird die Nachfrage des Patienten allerdings maßgeblich durch den Anbieter, in diesem Fall den behandelnden Arzt, bestimmt. Zudem besteht ein positiver Zusammenhang zwischen der Inanspruchnahme medizinischer Leistungen und der Ärztedichte, so dass beispielsweise auch Fahrt- und Wartezeiten zu berücksichtigen sind. In einem solchen Fall wäre der Konsum von Gesundheitsleistungen zur eingesetzten Zeit nicht mehr substitutiv, sondern komplementär, was in der Zeitallokationsentscheidung $\mathrm{zu}$ berücksichtigen wäre (vgl. Muurinen (1982) und Wagstaff

52 Ehrlich und Chuma (1990) weisen zudem darauf hin, dass mit der Lebenslänge auch die Zeitpräferenz für den Konsum beeinflusst wird, weshalb die Autoren eine Trennung in ein reines Investitions- und ein reines Konsummodell aus konzeptioneller Hinsicht nicht für sinnvoll erachten. $\mathrm{Zu}$ dem Trade-off von Lebenslänge und Lebensqualität unter Gesundheitsaspekten siehe darüber hinaus Eisenring (2000). 
(1986)).$^{53}$ Von jeglichen Anpassungskosten über die direkten Investitionskosten hinaus wird jedoch abstrahiert, weshalb Individuen ihren gewünschten Gesundheitskapitalbestand im Modell ohne zeitliche Verzögerung erreichen können. Über diese Annahme lässt sich auch begründen, warum die Theorie der abgeleiteten Nachfrage nach Gesundheitsleistungen in empirischen Untersuchungen teilweise nicht bestätigt werden kann (vgl. z. B. Wagstaff (1986) und Leu und Gerfin (1992)). ${ }^{54}$

Ein weiteres Zeitinkonsistenzproblem ergibt sich aus der Definition der Abschreibungen, die im Grossman-Modell ausschließlich altersabhängig ausgestaltet sind. Von der Möglichkeit gesundheitsschädigenden Verhaltens, beispielsweise durch Tabak- oder Alkoholkonsum oder eine ungesunde Ernährung, wird demnach ebenso abstrahiert wie von einem möglichen positiven Einfluss der Bildung auf eben dieses Verhalten. Bestimmt hingegen die Intensität der Nutzung des Gesundheitskapitalstocks die Abschreibungen, so dass beispielsweise der Lebensstil, Umwelteinflüsse oder auch das Bildungsniveau als Einflussgrößen herangezogen werden, müsste die Nachfrage nach Gesundheitsleistungen entsprechend steigen (vgl. z. B. Erbsland et al. (1998)). Da sich diese Annahme allerdings zumindest bei Wagstaff (1986) in der Empirie nicht bestätigt, kann es sein, dass sich in der Realität Zeitverzögerungen zwischen dem Verhalten und dem tatsächlichen Gesundheitszustand ergeben.

Zuletzt sind noch die Effekte der Bildung sowie eines steigenden Lohnsatzes kritisch zu beurteilen. Im Grossman-Modell erfolgt die Anhebung des Lohnsatzes ausschließlich exogen, so dass Bildung zumindest für das Einkommen keine explizite Rolle spielt. Zudem führt ein steigender Lohnsatz zu einer Rechtsverschiebung der Grenzertragskurve, während die Kosten des Gesundheitskapitals unverändert bleiben. Damit steigt die Nachfrage nach Gesundheit und entsprechend auch die Nachfrage nach Gesundheitsleistungen, denn je höher der Lohnsatz ist, desto wertvoller wird die verfügbare Arbeitszeit, weshalb Zeit gegen medizinische Leistungen substituiert wird. ${ }^{55}$ Aufgrund der unterstellten konstanten Skalenerträge ist diese Substitutionselastizität gleich Eins, so dass der Zeit und den Gesundheitsleistungen eine identische Produktivität beigemessen wird (vgl. Wagstaff (1986)). Je mehr Zeit jedoch in den Arbeitsmarkt investiert wird, desto weniger steht für die Investition in die Gesundheit zur Verfügung. Dementsprechend steigen die Zeitkosten der Investitionen, woraus sich wiederum ein

\footnotetext{
${ }^{53}$ Siehe zu diesen Zusammenhängen zudem Breyer et al. (2005).

${ }^{54}$ Neueren Studien zufolge (vgl. Gerdtham und Johannesson (1999) oder Nocera und Zweifel (1998)) kann die These der abgeleiteten Nachfrage hingegen bestätigt werden.

${ }^{55}$ Den Zusammenhang zwischen der Nachfrage nach Gesundheit und dem Lohnsatz beschreiben unter anderem auch Andrén und Palmer (2001).
} 
Rückgang der Nachfrage ergeben würde. Da Zeit jedoch nicht der einzige Inputfaktor in der Gesundheitsproduktion ist, ist der Anstieg des Lohnsatzes stets größer als der Anstieg der Investitionskosten. Die genaue Größe der gesundheitsfördernden Effekte einer Lohnsatzsteigerung hängt somit von der Elastizität der Nachfrage nach Gesundheit sowie der Zeitaufteilung im Moment der Lohnsatzerhöhung ab (vgl. McGuire et al. (1997), S. 140). Die Opportunitätskosten einer Einheit Zeitaufwand entsprechen im Grossman-Modell exakt dem Lohnsatz. Muurinen (1982) kritisiert hieran, dass der Lohnsatz genau dann kein geeignetes Maß für den Preis der aufgewendeten Zeit ist, sobald Arbeitszeit über das Einkommen hinaus Nutzen stiftend ist bzw. für Investitionen in einen der drei Kapitalbestände Gesundheit, Bildung und physisches Kapital verwendet werden kann. ${ }^{56}$

Der Effekt zunehmender Bildung ist ebenso ambivalent zu sehen: Mit steigender Bildung steigt zwar auf der einen Seite auch die Effizienz, mit der beispielsweise medizinische Leistungen nachgefragt werden. Es ist anzunehmen, dass zunehmend medizinisches Wissen und Wissen über alternative Ressourcen zur Verfügung steht, so dass die Inanspruchnahme medizinischer Leistungen zielgerichteter und aufgrund eines gesteigerten kooperativen Verhaltens der Patienten effektiver erfolgen kann. Grossman zufolge geht die Inanspruchnahme dieser Leistungen daher mit zunehmender Bildung zurück. Auf der anderen Seite wäre jedoch auch ein Nachfrageanstieg plausibel, da mit zunehmender Bildung auch der Lohnsatz steigt. Entsprechend höher sind die Einkommensverluste im Falle einer Krankheit, so dass Bildung mit steigenden Opportunitätskosten einhergeht und die Gesundheit relativ wertvoller wird (vgl. Muurinen (1982)). McGuire et al. (1997) geben darüber hinaus allerdings zu bedenken, dass eine Verbesserung der Gesundheit per se eine geringere Nachfrage nach Gesundheitsleistungen mit sich bringt, so dass die Inanspruchnahme von Gesundheitsleistungen für eine Elastizität kleiner Eins zurückgeht (vgl. S. 140). ${ }^{57}$

\subsection{Zur Bedeutung von Einkommen und Bildung für die Nachfrage nach Ge- sundheitsleistungen}

\subsubsection{Gesundheitsrelevantes Konsumverhalten}

Neben den direkten Investitionen in die Gesundheit spielt auch das individuelle Verhalten eine Rolle, das nicht primär auf die Gesundheit ausgerichtet ist. Ent-

\footnotetext{
${ }^{56}$ So wird durch ein gesteigertes Engagement am Arbeitsmarkt beispielsweise das jobspezifische Wissen vermehrt. Ebenso kann ,Learning by doing' zu einer gesteigerten Produktionseffizienz führen, wodurch ein steigendes Einkommen zu erwarten ist, da der Lohnsatz Ausdruck der Grenzproduktivität ist.

${ }^{57} \mathrm{Zu}$ weiteren kritischen Anmerkungen siehe bspw. Ried (1998b).
} 
spricht das Erwerbseinkommen dem Preis gesund verbrachter Zeit, dann werden Krankheiten oder Verletzungen um so eher vermieden, je höher das Erwerbseinkommen ist. Präventive Maßnahmen wie Impfungen oder Vorsorgeuntersuchungen werden eher durchgeführt. ${ }^{58}$

Eine wesentliche Bedeutung für die Entwicklung der Gesundheit kommt zudem der Ernährung zu. Solange nicht ausreichend Geld vorhanden ist, um zumindest die Grundversorgung zu ermöglichen, wirkt sich das fehlende Einkommen direkt negativ auf die Gesundheit aus. Es kommt zu Mangelerscheinungen oder sogar zu Unterernährung. Die weit reichende Konsequenz hieraus ist eine erhöhte Morbidität sowie ein erhöhtes Risiko der Arbeitsunfähigkeit, so dass das Einkommen nachhaltig negativ betroffen ist. Ein Verlassen dieses EinkommenGesundheits-Kreislaufs wird ohne Hilfe von außen unmöglich (vgl. Khan (1984)). Neben der negativen Beeinflussung des Immunsystems kann eine mangelhafte Ernährung zudem dazu führen, dass der Regenerationsprozess im Verlauf einer Krankheit nur langsamer voranschreitet, so dass letztlich mehr Zeit krank verbracht wird (vgl. Foster (1994)). ${ }^{59}$

Während Unterernährung insbesondere in ärmeren Ländern anzutreffen ist, existiert in Ländern mit einem hohen Bruttoinlandsprodukt häufig das Problem der Fehlversorgung durch minderwertige Nahrungsmittel, die in zu großen Mengen konsumiert werden und somit zu Fettleibigkeit führen (vgl. Bhattacharya et al. (2004), S. 857). Insbesondere Nahrungsmittel mit geringem Nährstoffgehalt sind schnell und günstig $\mathrm{zu}$ beschaffen und $\mathrm{zu}$ verzehren. Diese Entwicklung wirkt sich maßgeblich auf die Gesundheit von Kindern aus, da diese auf die Hilfe der Eltern angewiesen sind. Sind beide Eltern aufgrund eines zu niedrigen Familieneinkommens erwerbstätig oder aber ist ein allein erziehendes Elternteil auf das Erwerbseinkommen angewiesen, reicht die Zeit oftmals nicht aus, sich ausreichend um die Ernährung der Kinder zu kümmern. Dieser Effekt wird durch fehlende Bildung noch verstärkt, da nicht genügend Informationen über eine gesunde Ernährung vorhanden sind.

Sowohl für die Unter- als auch für die Überernährung gilt gleichermaßen, dass sich eine Erhöhung des Einkommens positiv auf die Gesundheit auswirkt. In

\footnotetext{
${ }^{58}$ Lee (1982) zeigt bspw. empirisch, dass ein hoher Lohnsatz die Nachfrage nach Gesundheit signifikant steigert.

${ }^{59}$ Um die Erwerbstătigkeit zu sichern, werden Nahrungsmittel hăufig innerhalb einer Familie von den Frauen zu den Männern und von Mädchen zu Jungen umverteilt (vgl. Pitt et al. (1990)). Ein derartiges Verhalten wirkt sich nachhaltig negativ auf die nachfolgenden Generationen aus, da sich der Gesundheitszustand in der Kindheit auf die Gesundheit wăhrend des ganzen Lebenszyklus auswirkt (vgl. Smith (1999)). Hieraus ergeben sich wiederum negative Effekte auf die Gesundheit potentieller Mutter und deren Kinder (vgl. Khan (1984)).
} 
dem ersten Fall können mehr Nahrungsmittel beschafft werden, während in dem zweiten Fall die Qualität der erworbenen Nahrungsmittel ansteigt. In beiden Fällen können sich hieraus zudem positive Produktivitätseffekte ergeben, die sich wiederum in Einkommenssteigerungen auswirken können.

Je höher das Einkommen ist, desto mehr Geld kann ebenso in die häusliche Umgebung investiert werden. Hierzu zählt insbesondere in Entwicklungsländern die Möglichkeit, sauberes Wasser zu bekommen oder aber über eine feste Behausung und entsprechende sanitäre Anlagen zu verfügen (vgl. Pritchett und Summers (1996)). Darüber hinaus sind generell Grundstücke, die durch weniger Umwelt- und insbesondere Luftverschmutzung belastet sind, teurer, so dass sich hier ein soziales Gefälle deutlich im jeweiligen Gesundheitszustand niederschlägt. Insofern kann eine Verbesserung der Gesundheit nicht nur durch die Finanzierung medizinischer Leistungen erreicht werden, sondern auch durch öffentliche Investitionen, beispielsweise im Hinblick auf den Umweltschutz sowie auf die sanitäre Versorgung (vgl. Leibowitz (2004), S. 668). Die entsprechenden Nachteile im Umfeld können daher besonders bei Menschen mit niedrigem Einkommen dazu führen, dass diese häufiger krank werden, da sie stärkeren Belastungen ausgesetzt sind (vgl. Currie und Stabile (2003), S. 1816).

Ein weiterer Kanal, über den sich das Einkommen indirekt auf die Gesundheit auswirkt, besteht in den Arbeitsbedingungen (vgl. Pitt et al. (1990)). Arbeiten, die mit einem geringen Lohnsatz und damit mit einem geringen Erwerbseinkommen einhergehen, sind häufig an körperliche Leistungsfähigkeit gekoppelt, so dass sich derartige Arbeitsbedingungen dauerhaft negativ auf die Gesundheit auswirken. Bei gleichzeitiger Unterernährung können die Arbeiten zudem nicht mit einer entsprechenden Produktivität verrichtet werden, so dass sich der Lohnsatz entsprechend nach unten anpasst. Letztlich ist ein Verlassen dieser Abwärtsspirale aus eigener Kraft kaum möglich, da zusätzliches Einkommen, das für eine Verbesserung der Ernährung unabdingbar ist, nur über eine gesteigerte Produktivität erreicht werden kann. Hierfür ist aber gerade eine bessere Gesundheit Voraussetzung. Geht diese Art der Erwerbstätigkeit also mit Unterernährung einher, dann kann eine Verbesserung der Ernährung dazu führen, dass die körperliche Arbeit leichter und mit höherer Produktivität verrichtet werden kann, was sich wiederum positiv auf das Einkommen auswirkt. Ohne das notwendige Einkommen ist eine Verbesserung der Ernährung aus eigener Kraft jedoch nicht möglich.

Nicht eindeutig ist sicherlich der Zusammenhang zwischen Nikotin- und Alkoholkonsum auf der einen und dem Einkommen auf der anderen Seite. Zwar wird bei hohem Einkommen aufgrund der hohen Opportunitätskosten eher von gesundheitsschädigendem Verhalten abgesehen, physischer sowie psychischer 
Stress können jedoch dazu führen, dass der Konsum entsprechend höher ausfällt. $^{60}$

\subsubsection{Die Bedeutung der Bildung für die Gesundheit}

Eine wesentliche Komponente für ein höheres Einkommen ist Bildung, so dass sich hierüber positive Gesundheitseffekte ergeben können. Die Kausalität zwischen den Humankapitalbestandteilen Gesundheit und Bildung geht damit in beide Richtungen, d.h. Gesundheit beeinflusst die Bereitschaft, in die eigene Ausbildung zu investieren, indem diese sowohl den Anreiz zu Schulbildung erhöht als auch die Produktivität steigert und somit den notwendigen Zeitaufwand senkt. Für den positiven Einfluss der Bildung auf die Gesundheit lassen sich folgende Argumente anführen: In der Regel ist die Produktivität von Individuen mit einer weit reichenden Ausbildung größer als bei denjenigen, die keine Ausbildung absolviert haben. Aus einer gegebenen Menge an Inputgütern kann ein größerer Output produziert werden. Dieser Zusammenhang lässt sich auch auf die Investitionen in die Gesundheit übertragen, d. h. die Transformation von Gesundheitsleistungen in Gesundheitskapital kann mit einer höheren Produktionseffizienz durchgefuhrt werden (vgl. hierzu Grossman (2000), S. 396). Gemäß dieser These sind für einen identischen Gesundheitskapitalstock weniger Gesundheitsgüter notwendig, was als direkter Effekt der Bildung auf die Gesundheit bezeichnet werden kann. Der Einfluss der Bildung auf die Haushaltsproduktion kann folglich mit der Bedeutung des Technologieniveaus für die Unternehmensproduktivität verglichen werden.

Das zweite Argument für einen positiven Kausalzusammenhang in die andere Richtung bezieht sich auf eine gesteigerte Allokationseffizienz. Hierbei geht es nicht um den korrekten Einsatz von Gesundheitsgütern, sondern vielmehr um die Auswahl der verwendeten Gesundheitsleistungen, so dass dieser Effekt als indirekt bezeichnet werden kann. ${ }^{61}$

Ein drittes Argument bezieht sich schließlich auf den Lebenswandel. So nimmt Kenkel (1991) an, dass das Verständnis für gesundheitsrelevantes Verhalten und

${ }^{60}$ Zum Zusammenhang von Einkommen und Alkoholkonsum siehe bspw. Ettner (1996).

${ }^{61}$ Diese Aufspaltung in einen direkten und einen indirekten Effekt geht auf Welch (1970), S. 42, zurück. Welch unterscheidet einen Arbeitskrafteffekt und einen Allokationseffekt. Der erste Effekt beschreibt die Fähigkeit von Arbeitskräften, aus der gleichen Zahl an Inputgütern einen höheren Output zu erzielen, während der Allokationseffekt auf die Fähigkeit von Arbeitskräften abstellt, Informationen über die Kosten und Produktionscharakteristika anderer Inputgüter zu beschaffen, so dass sich eine Veränderung der Bildung verändernd auf die Menge der Inputs auswirkt. Hierzu zählt auch, dass neue Technologien nachgefragt und angewendet werden, die anderenfalls nicht in den Produktionsprozess eingingen. 
deren Konsequenzen für die Gesundheit ausgeprägter ist, je mehr Bildung vorhanden ist. Kenkel zeigt empirisch, dass Bildung einen statistisch signifikanten negativen Einfluss auf das Rauchen sowie den Alkoholkonsum ausübt, während der Einfluss auf ausgleichenden Sport signifikant positiv ist.

In einer Studie von Gilleskie und Harrison (1998) zeigt sich ebenfalls ein hoch signifikant positiver Effekt der Bildung auf die Gesundheit, indem die Produktionseffizienz ansteigt und so die Produktionskosten entsprechend zurückgehen. Darüber hinaus steigt auch der Allokationseffekt, indem Gesundheitsgüter höherer Qualität nachgefragt werden und gesundheitsschädigendes Verhalten umso eher vermieden wird, so dass sich Bildung hier indirekt auf die Gesundheit auswirkt. ${ }^{62}$

Im Rahmen einer empirischen Untersuchung zeigt Grossman (1975), dass erstens ein zusätzliches Schuljahr den Stundenlohnsatz um annähernd 5,5 Prozent erhöht und dass zweitens die Gesundheitsproduktivität um mindestens 2,4 Prozent ansteigt, so dass etwa 40 Prozent der gesamten Produktivitätssteigerungen bezüglich der Gesundheitsinvestitionen auf die Bildung zurückgeführt werden können (vgl. hierzu Grossman (1975), S. 179). Insgesamt werden knapp zwei Drittel des positiven Einflusses der Bildung auf die Gesundheit durch den oben genannten direkten Effekt abgebildet, während die indirekte Komponente ein Drittel der zusätzlichen Produktivität erklärt (vgl. Grossman (1975), S. 185). Eine Erhöhung der für Bildungszwecke verfügbaren Ressourcen würde den allgemeinen Gesundheitsstatus effektiv erhöhen, indem die Wahl der Gesundheitsinputs verändert würde.

Berger und Leigh (2001) beschäftigen sich mit der Frage, ob tatsächlich die Produktionseffizienz der Gesundheit durch eine höhere Bildung gesteigert wird oder ob unbeobachtete Variablen, beispielsweise genetische Faktoren oder die Diskontierungsrate, Bildung und Gesundheit in gleicher Richtung beeinflussen. Wäre dies der Fall, dann würde ein höheres Bildungsniveau nicht zu einer verbesserten Gesundheit führen. In ihrer Arbeit kommen Berger und Leigh allerdings ebenfalls zu dem Ergebnis, dass der Zusammenhang zwischen Bildung und Gesundheit insbesondere durch den direkten Einfluss der Bildung auf den Gesundheitsproduktionsprozess abgebildet wird und nicht durch unbeobachtete Variablen. Als Implikation für die Politik ergibt sich hieraus, dass Bildungspro-

${ }^{62}$ Ökonomische Anreize für ein gesundheitsförderndes Verhalten von Menschen mit hohem Bildungsabschluss ergeben sich aus den hiermit verbundenen hohen Opportunitätskosten. Diesen Zusammenhang finden Gilleskie und Harrison (1998) für Frauen bestätigt, während die Wahrscheinlichkeit einer guten Gesundheit für Männer nicht so deutlich von der Bildung abhängt. 
gramme genutzt werden können, um die Gesundheit der gesamten Bevölkerung zu verbessern (vgl. Berger und Leigh (2001), S. 447).

Die bislang genannten Wirkungszusammenhänge zwischen Bildung auf der einen und Gesundheit auf der anderen Seite bezogen sich sämtlich auf die gezielte Verbesserung der eigenen Gesundheit. Darüber hinaus ist ebenfalls zu berücksichtigen, wie sich die Bildung von Eltern auf die Gesundheit der Kinder auswirkt und welche Entwicklungsmöglichkeiten sich hieraus ergeben.

Zunächst wirkt sich Bildung auf die Bevölkerungsentwicklung aus, wie Schultz (1999) zeigt. So lassen sich Unterschiede in der Lebenserwartung zwischen einzelnen Ländern auf die Sterbewahrscheinlichkeit von Erwachsenen sowie auf die Säuglings- und Kindersterblichkeit zurückführen. Eltern mit einem höheren Bildungsabschluss können über ihr Verhalten nicht nur die eigene Lebenslänge positiv beeinflussen, sondern darüber hinaus auch die Überlebenschancen ihrer Nachkommen erhöhen, indem sie über mehr gesundheitsrelevantes Wissen verfügen und so die Kinder unter gegebenen Umständen optimal versorgen können. Darüber hinaus ist Bildung mit einem steigenden Einkommen verbunden, so dass Gesundheitsleistungen bezahlbar werden.

Eine sinkende Kindersterblichkeit ist gleichzeitig Ursache für große Unterschiede in den gesamtwirtschaftlichen Wachstumsraten zwischen einzelnen Ländern. Für den Fall, dass Eltern diese Entwicklung nicht zeitnah bemerken, steigt allein die Kinderzahl, so dass pro Kind immer weniger Einkommen zur Verfügung steht. Alternativ kann jedoch auch die Fertilität sinken, wenn Eltern entsprechend auf die verbesserte Gesundheit der Kinder reagieren (vgl. KalemliOzcan et al. (2000), S. 2). Hieraus folgt zudem, dass bei einer gleichen absoluten Ausgabenhöhe für Bildungszwecke jedem Kind relativ ein höherer Betrag zugute kommt, so dass sich hieraus wiederum ein höheres Bildungsniveau entwickeln kann (vgl. Zhang (2003), S. 1826).

\subsubsection{Zugang zu Gesundheitsleistungen}

Das Einkommen beeinflusst nicht nur die Bereitschaft, in die eigene Gesundheit zu investieren, sondern auch das Budget, das für derartige Investitionen zur Verfügung steht. Es entstehen sowohl direkte als auch indirekte Kosten aus den Gesundheitsinvestitionen. $\mathrm{Zu}$ den direkten Kosten zählen die direkten privaten Ausgaben für medizinische Leistungen, so dass hier der Preis der Leistungen die Nachfrage steuert. Je teurer ein Medikament ist und je mehr davon privat zu tragen ist, desto größer sind die negativen Verteilungswirkungen. Menschen mit geringem Einkommen können unabhängig von ihrem Bedarf die notwendigen medizinischen Leistungen nicht konsumieren. Daher ist der negative Zusammenhang zwischen einem niedrigen Einkommen und der Gesundheit umso stär- 
ker, je größer der finanzielle Eigenanteil an den Gesundheitsleistungen im Verhältnis zum gesamten Einkommen ist.

In einem System ohne Krankenversicherung besteht das Problem, dass medizinische Leistungen vollständig privat finanziert werden müssen. Ist entsprechendes Vermögen vorhanden, können Gesundheitsschocks ${ }^{63}$ finanziell aufgefangen werden (vgl. Over et al. (1992), S. 189). Ohne derartiges Vermögen wären Kredite notwendig, um die notwendige medizinische Versorgung $\mathrm{zu}$ finanzieren. Hier zeigt sich erneut der negative Einkommens-Gesundheits-Kreislauf, da Kredite nur dann vergeben werden, wenn entsprechende Sicherheiten wie beispielsweise Grundbesitz vorhanden sind. Je höher der Preis für die notwendigen Gesundheitsgüter ist, desto eher ist es insbesondere armen Familien nicht möglich, diese zu beschaffen.

Zusätzlich zu den direkten Kosten von Gesundheitsinvestitionen sind auch indirekte Kosten wie Fahrt- und Wartezeiten zu berücksichtigen. Insbesondere in Gebieten mit einer geringen Arztdichte sind Fahrtkosten ebenfalls in ihrer negativen Wirkung nicht zu unterschätzen. Hier ergibt sich sowohl für niedrige als auch für hohe Einkommen ein negativer Anreizeffekt. Je höher das individuelle Einkommen ist, desto eher kann es sein, dass Zeit gegen medizinische Leistung substituiert wird, ohne dass vorher eine Arztkonsultation stattgefunden hat. Bei sehr niedrigen Einkommen bedeutet der Zeitaufwand hingegen gleichzeitig einen Verlust des lebensnotwendigen Erwerbseinkommens, so dass weniger medizinische Leistungen und damit auch weniger Gesundheit nachgefragt werden.

Problematisch ist ein begrenzter Zugang zu Gesundheitsleistungen insbesondere dann, wenn aus diesem Grund präventive Maßnahmen unterlassen werden. Besonders in ärmeren Ländern leben viele Menschen auf engstem Raum beieinander, so dass sich Krankheiten leichter übertragen (vgl. Over et al. (1992), S. 165). Solange nicht ausreichend finanzielle Mittel zur Verfügung stehen, um die medizinischen Leistungen für alle Familienmitglieder zu bezahlen, kann dieser negative Gesundheitseffekt nicht vermieden werden.

Allgemein ist die Frage zu beantworten, ob tatsächlich der Zugang zu Gesundheitsleistungen für ärmere Familien erschwert ist oder ob die Mitglieder dieser Familien zwar einen ähnlichen Genesungsprozess durchlaufen, jedoch häufiger Gesundheitsschocks ausgesetzt sind. Currie und Stabile (2003) kommen in einer empirischen Überprüfung anhand kanadischer Mikrodaten zu dem Ergebnis, dass die Erkrankungswahrscheinlichkeit von Kindern aus niedrigeren Einkom-

${ }^{63}$ Unter Gesundheitsschocks sind Veränderungen in der Gesundheit zu verstehen, die nicht auf das eigene Verhalten zurückzuführen und damit unvorhersehbar sind. 
mensschichten höher ist als bei Kindern, deren Eltern über ein höheres Einkommen verfügen, während sich langfristig keine Auswirkungen auf die $\mathrm{Ge}-$ sundheit ergeben. Dieser Zusammenhang kann beispielsweise auf unzureichende Ernährung oder aber auf weniger präventive Gesundheitsleistungen zurückgeführt werden. Zumindest für Kanada muss die Hypothese des beschränkten Zugangs zu Gesundheitsleistungen daher verworfen werden. Dennoch kann diese insbesondere in Ländern mit einem unzureichend entwickelten Gesundheitssystem weiterhin Bestand haben - hier wären weitere empirische Studien von besonderem Interesse. 


\section{Gesundheit und Arbeitsangebot}

\subsection{Die Bedeutung der Arbeit für Gesundheitsinvestitionen}

\subsubsection{Grundüberlegungen}

In den vorangegangenen Kapiteln wurden zahlreiche Wege aufgezeigt, wie Gesundheit Wirtschaftswachstum fördert, indem das effektive Arbeitsangebot verbessert wird. Die Gesundheit determiniert die insgesamt verfügbare Arbeitszeit sowie die Produktivität, während Krankheit und damit Erwerbsunfähigkeit das Arbeitseinkommen negativ beeinflussen.

Des Weiteren wird auch zukünftiges Arbeitseinkommen durch schlechte Gesundheit vergangener Perioden aufgrund geringerer Produktivitätszuwächse negativ beeinflusst (vgl. Chirikos und Nestel (1985), S. 65). Eine schlechte Gesundheit führt folglich nicht nur für das einzelne Individuum, sondern auch gesamtwirtschaftlich zu hohen Kosten. Es entstehen direkte Kosten infolge von Krankheit, die sich aus dem Krankheitsschaden und dem Ressourceneinsatz für die Behandlung ergeben, so dass diese Ressourcen alternativen Verwendungszwecken entzogen werden. Zudem entstehen indirekte Kosten, indem erstens ein zu geringes Arbeitsangebot einen Verlust an Output mit sich bringt und zweitens ein Verlust an Humankapital zu geringeren zukünftigen Produktionsergebnissen führt (vgl. Pauly et al. (2002), S. 222).

Darüber hinaus beeinflusst gerade das individuelle Arbeitsangebot die Investitionen in die Gesundheit, so dass auch eine Kausalbeziehung in die andere Richtung existiert. Insgesamt sind hier drei Wirkungskanäle zu nennen: Erstens sind Investitionen, beispielsweise Arztbesuche, in der Regel mit Zeitaufwand verbunden. Somit schließen sich lange Arbeitszeiten sowie ein großer Zeitaufwand für Gesundheitszwecke gegenseitig aus, so dass Arbeit hier einen negativen Einfluss ausübt. Zweitens determiniert Erwerbstätigkeit das verfügbare Einkommen. Für einen gegebenen Lohnsatz ist das Einkommen umso größer, je länger gearbeitet wird. Über ein hohes Einkommen können wiederum Gesundheitsgüter, seien es medizinische Leistungen oder qualitativ hochwertige Nahrungsmittel, beschafft werden. Daher wirkt sich Arbeit als Determinante des Einkommens positiv auf die Gesundheit aus.

Der dritte Effekt, der mit Arbeit verbunden ist, betrifft die Abschreibungen. Hier spielen insbesondere die Arbeitsbedingungen eine große Rolle. Besonders bei unqualifizierter Arbeit besteht ein Trade-off zwischen dem Einkommen auf der

${ }^{64} \mathrm{Zu}$ den direkten und indirekten Kosten von Krankheiten siehe auch Henke et al. (1997). 
einen und der Gesundheit auf der anderen Seite, da unqualifizierte Arbeit häufig mit körperlicher Anstrengung einhergeht und so die Abschreibungen auf die Gesundheit erhöht (vgl. hierzu Leigh (1983) und Kemna (1987)).

Zudem kann Arbeit die Abschreibungsrate auch positiv beeinflussen, indem sich hierüber eine größere Zufriedenheit einstellt und die Gesundheit an Bedeutung gewinnt. Krank verbrachte Zeit wirkt sich negativ auf das gegenwärtige Einkommen sowie die Einkommensentwicklung aus, so dass eher von gesundheitsschädigendem Verhalten abgesehen wird.

\subsubsection{Empirische Studien}

Eine Reihe empirischer Studien befasst sich mit dem Zusammenhang zwischen der Arbeitszeit auf der einen und der Gesundheit auf der anderen Seite, wobei in der Regel auf Überstunden sowie lange Arbeitszeiten Bezug genommen wird. Mathers (1994) (zit. in Schofield (1996), S. 3) zeigt beispielsweise, dass Arbeitskräfte mit einer Arbeitszeit von über 25 Stunden pro Woche zwar prinzipiell gesunder, d.h. seltener krank sind, aber seltener Sport treiben und zudem einen höheren Alkoholkonsum aufweisen. Schofield (1996) zufolge sind Personen mit hohen Arbeitszeiten zudem eher Raucher, suchen andererseits aber seltener den Arzt auf. Während die ersten Ergebnisse im Wesentlichen auf mit der Arbeit verbundenen Stress zurückgeführt werden können, könnten die selteneren Arztbesuche ein Indikator für die Zeitrestriktionen sein, mit denen entsprechende Arbeitszeiten verbunden sind. Erstens sind Arztbesuche in der Regel zeitintensiv, so dass diese bei hohen regulären Arbeitszeiten nach Möglichkeit vermieden werden, und zweitens bringt häufige Krankheit negative berufliche Konsequenzen mit sich. So steigt die Wahrscheinlichkeit, arbeitslos zu werden, mit häufiger Krankheit. Gleichzeitig werden Personen mit schlechter Gesundheit nicht so schnell wieder eingestellt (vgl. Stewart (2001), S. 782f.).

$\mathrm{Zu}$ ähnlichen Ergebnissen kommen Spurgeon et al. (1997). Bei Männern mit langen Arbeitszeiten ist die Wahrscheinlichkeit zu rauchen oder eines ausgeprägten Alkoholkonsums größer als bei Männern mit regulären Arbeitszeiten. Darüber hinaus steigt die Gefahr von Übergewicht, obwohl sich in dieser Studie keine negativen Auswirkungen auf die sportliche Betätigung ergeben.

Neben dem ungesunden Verhalten gehen weitere Stressfaktoren mit langen Arbeitszeiten einher. Hierunter sind Beeinträchtigungen im Bewegungsapparat, Magen-Darm-Erkrankungen und Herzinfarkte ebenso zu fassen wie psychische Probleme, Schlafstörungen und Kopfschmerzen (vgl. Spurgeon et al. (1997) und Sparks et al. (1997)). Diese Effekte wirken sich negativ auf die individuelle Produktivität aus und senken so die erbrachte Arbeitsleistung. Durch die mit langen 
Arbeitszeiten einhergehende abnehmende Konzentration sinkt zudem die Arbeitssicherheit, woraus sich wiederum Gesundheitsrisiken ergeben können. ${ }^{65}$

Welche negativen Konsequenzen mit arbeitsbedingter schlechter Gesundheit einhergehen können, zeigt ein Blick in den japanischen Wirtschaftsraum. Hier existiert eigens der Begriff Karoshi, was Tod durch Überarbeitung bedeutet (vgl. Nishiyama und Johnson (1997)). Dauerhafte Überstunden und Schichtarbeit verursachen hiernach extremen Stress und können sogar zum Tod führen, indem für Arztbesuche und anderweitige medizinische Betreuung nicht mehr ausreichend Zeit zur Verfügung steht. 1988 arbeiteten der Studie von Nishiyama und Johnson (1997) zufolge 24 Prozent der männlichen Arbeiter mehr als 60 Stunden pro Woche.

Das Karoshi-Phänomen ist unter anderem auch Gegenstand der Arbeit von Leontaridi und Ward (2002). In deren Studie zeigt sich, dass Stress signifikant mit Bildung, den individuellen Fähigkeiten sowie dem Einkommensniveau zunimmt. Diese Größen sind maßgebliche Indikatoren für die berufliche Verantwortung, die ein Beschäftigter übernimmt. Dementsprechend gibt es einige $\mathrm{Be}$ rufsgruppen, die besonders stressanfällig sind, beispielsweise Polizisten, so dass die Gesundheit hier besonders stark in Mitleidenschaft gezogen wird.

Derartige Probleme sind nicht nur für die einzelnen Unternehmen, sondern für die gesamte Gesellschaft mit hohen Kosten verbunden. Hierzu gehören insbesondere Fehlzeiten sowie eine geringe Produktivität als Konsequenz aus einer schlechten Gesundheit. Gleichzeitig steigt jedoch das Risiko von Arbeitsunfällen, und auch häufige Arbeitsplatzwechsel resultieren hieraus, so dass sich zu hohe Arbeitszeiten sowie schlechte Arbeitsbedingungen mehrfach negativ auf die gesamtwirtschaftliche Entwicklung auswirken (vgl. Leontaridi und Ward (2002)).

Nichtsdestotrotz besteht in der Literatur Uneinigkeit über den Einfluss von Arbeitszeiten auf die Gesundheit. Sundberg (1996) bestätigt den negativen Effekt

${ }^{65}$ Inwiefern sich tatsächlich höhere Gesundheitsrisiken ergeben, ist maßgeblich von der Art der beruflichen Tätigkeit abhängig. Die Gefahr eines Berufsunfalls ist bei körperlichen Tätigkeiten sicherlich höher als bei rein administrativen Aufgaben, die nahezu ausschließlich im Büro erledigt werden. In manchen Branchen sind zudem andere Personen negativ betroffen. Als Beispiel können hier Patienten von unkonzentrierten Ärzten angeführt werden. Andererseits können sich lange Arbeitszeiten sogar positiv auf die Gesundheit auswirken, wenn hiermit eine Verbesserung der Arbeitsbedingungen einhergeht. Ist es in einem Büro während der Überstunden merklich ruhiger als in der regulären Arbeitszeit, erhöht sich noch einmal die Produktivität, Stress kann so reduziert werden. Folglich sind die Arbeitsbedingungen bei der Bewertung zusätzlicher Arbeitszeit zu berücksichtigen (vgl. Spurgeon et al. (1997)). 
langer Arbeitszeiten, während Haveman et al. (1994) davon ausgeht, dass nicht die Arbeitszeit, sondern die Arbeitsbedingungen relevant sind. Letztlich handelt es sich hierbei jedoch um ein sehr subjektives Kriterium, das mögliche andere Ursachen für persönliche Unzufriedenheit überdecken könnte. Auch in der Arbeit von Sundberg (1998), in der sich die Arbeitszeit nur auf die Gesundheit von Frauen negativ auswirkt, ist der Einfluss der Arbeitsbedingungen nicht eindeutig.

\subsection{Der Zusammenhang zwischen medizinischen Leistungen und Zeitaufwand}

Grossman (1972) beschreibt Individuen als Produzenten ihrer Gesundheit, indem sie unter Verwendung von Zeit Gesundheitsleistungen nachfragen und dadurch ihren eigenen Gesundheitskapitalstock erhöhen. Grundlegendes Ziel ist hierbei die Beeinflussung des individuellen Nutzens einerseits direkt über die Gesundheit, andererseits indirekt über das Einkommen und damit über das Konsumniveau. Gegenstand der folgenden Analyse ist die Annahme, dass Individuen Zeit und Einkommen in ihre Gesundheit investieren, wobei an dieser Stelle nicht die Nutzenstiftung der Gesundheit für den Einzelnen Gegenstand des Interesses ist, sondern der Einfluss der Arbeitsangebotsentscheidung auf die Gesundheit und vice versa. Im Gegensatz zu dem Modell Grossmans wird im folgenden Modell nicht der Nutzen maximiert, sondern die für die Gesundheit optimale Zeitallokation bestimmt.

Unter Investitionen in die Gesundheit wird sämtlicher Konsum von Gütern subsumiert, welche in ihrem Charakter einem Gesundheitsgut entsprechen und folglich einen positiven Einfluss auf die Gesundheit ausüben. Hierzu zählen neben den medizinischen Leistungen beispielsweise ein gesunder Lebensstil, d. h. sportliche Betätigung und eine gesunde Ernährung, sowie eine ausreichende sanitäre Versorgung. Weiterhin wird angenommen, dass die Gesundheit analog zu physischem Kapital einer natürlichen Abschreibungsrate unterliegt, die durch die individuelle Arbeitsangebotsentscheidung sowie durch das Freizeitverhalten beeinflusst wird. ${ }^{66}$

Im Rahmen der Haushaltsproduktion kombiniert das Individuum stets Zeit mit Gesundheitsleistungen und bestimmt so die Investitionshöhe in das Gesundheitskapital, wobei weder auf Gesundheitsleistungen noch auf Zeitaufwand vollständig verzichtet werden kann. Im Rahmen einer Einzelbetrachtung lässt sich der Zusammenhang zwischen den Investitionskomponenten jedoch in drei Kate-

\footnotetext{
${ }^{66}$ Von gesundheitsschädigenden Konsumgütern wird im Folgenden aus Vereinfachungsgründen abstrahiert. Es ist jedoch ebenso denkbar, dass sich Freizeit erst unter Verwendung derartiger Konsumgüter negativ auf die Gesundheit auswirkt.
} 
gorien aufgliedern: Es ist erstens möglich, dass eine medizinische Leistung substitutiv zum Zeitaufwand nachgefragt werden kann, indem sich die Wirkungsweisen nicht voneinander unterscheiden. Für den Konsum dieser Güter ist kein direkter Zeitaufwand notwendig, vielmehr entsprechen sie einer Alternative $\mathrm{zu}$ der aufzuwendenden Zeit. Hierzu gehören insbesondere Medikamente, die eine Abschwächung des Gesundheitskapitals auf eine ähnliche Weise korrigieren wie körperliche Erholung, so dass im Fall einer Krankheit die Entscheidungsalternative zwischen dem Konsum von Gesundheitsgütern und dem Zeitaufwand besteht.

Die zweite Ausprägung der Investitionstätigkeit von Individuen bilden Gesundheitsgüter, die lediglich mit einem bestimmten Zeitaufwand konsumiert werden können (Komplemente). Es besteht folglich ein festes Einsatzverhältnis zwischen den beiden Inputgütern der Gesundheitsproduktion. $\mathrm{Zu}$ der Klasse der Komplemente gehören neben der Behandlung von Krankheiten, in die ein gewisses $\mathrm{Ma} ß$ an Behandlungszeit sowie Warte- und Fahrtzeit investiert werden muss, auch eine Reihe präventiver Maßnahmen, bspw. sportliche Betätigung, für deren Ausführung Marktgüter benötigt werden. ${ }^{67}$ Prävention wirkt in diesem Kontext dem negativen Einfluss der Abschreibungsrate entgegen mit dem Ziel, ein Absinken des Gesundheitskapitalstocks zu verhindern.

Die dritte Kategorie von Gesundheitsinvestitionen enthält sämtliche Leistungen, die den Gesundheitskapitalstock ohne nennenswerten Zeitaufwand erhöhen. Zu diesen Gütern gehören einmalige Investitionen in die Gesundheit, beispielsweise durch eine Verbesserung der Wohnsituation oder der sanitären Versorgung, ebenso wie Investitionen, die aus einer Verbesserung der Qualität der in Anspruch genommenen Gesundheitsgüter resultieren und aufgrund des zu erwartenden höheren Preises ein dauerhaft höheres Einkommen voraussetzen. Im Hinblick auf den direkten Umsetzungsprozess ergeben sich jedoch keine Konsequenzen für die Zeitallokation. ${ }^{68}$

Aus diesen Überlegungen folgt, dass eine geringere Arbeitszeit zu der Nachfrage nach Gesundheitsleistungen sowohl komplementär als auch substitutiv sein kann (vgl. Gilleskie (1998), S. 3). Je höher die Arbeitszeit indes ist, desto weniger Zeit steht für Investitionen zur Verfügung, so dass immer weniger Leis-

\footnotetext{
${ }^{67}$ Die Zeit, die eine Person zusätzlich zur reinen Gesundheitsleistung aufzuwenden bereit ist, bestimmt im Fall der Komplemente maßgeblich den Behandlungserfolg beziehungsweise den Erfolg präventiver Maßnahmen. Eine nähere Analyse des behandlungsbegleitenden Verhaltens von Individuen findet sich in Schneider (2002).

${ }^{68}$ In diesem Fall wird angenommen, dass sich z. B. keine Veränderung von Fahrtzeiten ergibt. Sollte dies der Fall sein, würde sich die Zeitallokation insofern ändern, als dass insgesamt weniger Zeit für Investitionen in die Gesundheit zur Verfügung steht.
} 
tungen in Anspruch genommen werden können, die mit direktem Zeitaufwand verbunden sind. Unter der Prämisse, dass sowohl substitutive als auch komplementäre Gesundheitsgüter für eine gute Gesundheit erforderlich sind, gewinnen letztere folglich mit zunehmender Arbeitszeit an Bedeutung.

\subsection{Die optimale Gesundheit in der kurzen Frist}

Nachfolgend wird ein Gesundheitsproduktionsmodell im Stil des GrossmanModells entwickelt, das sich insbesondere auf mögliche Arbeitszeiteffekte konzentriert. Zudem wird die Existenz einer Krankenversicherung nun explizit berücksichtigt. Aus den genannten Gründen wird im Rahmen des folgenden Modells angenommen, dass ein Teil der Gesundheitsgüter substitutiv zu der aufzuwendenden Zeit ist, während ein weiterer Teil als Komplemente aufzufassen ist. Im Fall der Substitute kann eine identische Investitionshöhe entweder durch Zeitaufwand oder aber mit einem verstärkten Einsatz an Gesundheitsleistungen erzielt werden. Weiter wird angenommen, dass die Gesundheit ohne Investitionen nicht auf konstantem Niveau verharrt, da das Gesundheitskapital analog zu anderen Formen des Humankapitals Abschreibungen unterliegt, die eine stetige Verschlechterung der Gesundheit nach sich ziehen.

Auch wenn die Gesundheit in dieser Form der Bildung als Humankapitalstock ähnlich ist, ist Gesundheitskapital für den Einzelnen grundsätzlich einfacher zu akkumulieren, da im Grunde keine besonderen persönlichen Fähigkeiten vorausgesetzt werden. Dennoch existieren analog zur Bildung auch bei der Akkumulation von Gesundheitskapital große Unterschiede zwischen einzelnen Individuen, da finanzielle Ressourcen, die die Nachfrage nach materiellen Gesundheitsgütern ermöglichen, sowie Informationen über gesundheitsförderndes Verhalten und damit Bildung für eine gute Gesundheit unabdingbar sind (vgl. Andrén und Palmer (2001), S. 11). Nimmt man in diesem Zusammenhang an, dass das verfügbare Wissen sowie der Lohnsatz eines Individuums kurzfristig konstant sind, besteht die einzige Möglichkeit, das Einkommen und damit die Nachfrage nach Gesundheit zu beeinflussen, in der Allokationsentscheidung in Bezug auf die Arbeitszeit. ${ }^{69}$

Beschreibt nun $H$ den individuellen Gesundheitskapitalstock, so hängt dieser im folgenden Modell positiv von den Investitionen in die Gesundheit ab. Diese las-

${ }^{69}$ Zusätzliche Informationen über gesundheitsförderndes Verhalten ermöglichen eine gesteigerte Produktions- sowie Allokationseffizienz im Hinblick auf die Gesundheitsinvestitionen, so dass sich ein höherer Gesundheitskapitalstock einmalig auch bei unverändertem Einkommen erzielen ließe. Im Rahmen des präsentierten Modells wird von derartigen Bildungseffekten abstrahiert. 
sen sich untergliedern in die Nachfrage nach Gesundheitsleistungen $M$ sowie die für die Investitionen notwendige Zeit $t_{M}$, nachfolgend Investitionszeit genannt. Ein negativer Einfluss auf die Gesundheit ergibt sich hingegen aus der Abschreibungsrate $\delta$. Es wird angenommen, dass ein konstantes Gesundheitsniveau sowohl über eine hohe Nachfrage nach Gesundheitsleistungen und damit einhergehend über einen geringen Zeitaufwand als auch über hohe Zeitinvestitionen bei geringer Leistungsnachfrage erreicht werden kann. Dementsprechend wird der Gesundheitskapitalstock $H$ durch

$$
\begin{aligned}
& H=H\left(M, t_{M}, \delta\right) \quad \text { mit } \quad H_{1}>0, H_{2}>0, H_{3}<0 \\
& \text { und } H_{11}<0, H_{22} \leq 0, H_{33} \geq 0
\end{aligned}
$$

bestimmt. $H_{1}$ beschreibt in diesem Zusammenhang die Ableitung der Gesundheitsfunktion nach den medizinischen Leistungen $\mathrm{M}, \mathrm{H}_{2}$ die Ableitung nach der Investitionszeit und $\mathrm{H}_{3}$ entsprechend nach den Abschreibungen. Darunter sind jeweils die zweiten Ableitungen angegeben. ${ }^{70}$ Für die Gesundheitsleistungen $M$ werden abnehmende Grenzerträge unterstellt, während der Einfluss des Zeitaufwandes $t_{M}$ konstant oder abnehmend sein kann. Der Einfluss der Abschreibungen ist entweder linear oder aber sogar zunehmend. ${ }^{71}$

Es wird angenommen, dass sich die Gesundheitsleistungen $M$ sowie die Investitionszeit $t_{M}$ strikt positiv auf die Gesundheit auswirken, d. h. der Konsum von Gesundheitsleistungen ist mit keinerlei Nebenwirkungen verbunden. Abschreibungen führen hingegen zu einem Absinken des Gesundheitskapitalstocks. Die für die Investitionen aufgewendete Zeit umfasst sämtlichen Zeitaufwand, der zum einen in Kombination mit Gesundheitsgütern einsetzbar ist (Komplemente), zum anderen jedoch auch direkt ohne den Konsum medizinischer Leistungen zu einer Verbesserung der Gesundheit führt. Der zweitgenannte Fall umfasst diejenige Investitionszeit, die ohne den dauerhaften Einsatz finanzieller Ressourcen Schwächungen im Gesundheitskapital korrigieren kann; so lässt sich eine Erkältung durch Ruhe auch ohne den Konsum von Medikamenten auskurieren. Weiterhin kann Investitionszeit beispielsweise für sportliche Aktivitäten aufgewendet werden, wobei hier der präventive Charakter im Vordergrund steht. Ein Absinken der Investitionszeit bedeutet c. p. eine Verschlechterung der Gesund-

\footnotetext{
${ }^{70}$ Über die Kreuzableitungen werden keine Annahmen getroffen. Zumindest für die Kreuzableitung nach $M$ und $t_{M}$ lässt sich jedoch sagen, dass diese ein positives Vorzeichen aufweist, wenn medizinische Leistungen und Investitionszeit im Wesentlichen Komplemente sind, während ein negatives Vorzeichen einen hauptsächlich substitutiven Zusammenhang beschreiben würde.

${ }^{71}$ Eine nähere Erläuterung hierzu folgt im Anschluss an Gleichung (5.9).
} 
heit, die über eine gesteigerte Nachfrage nach substitutiven Gesundheitsleistungen auszugleichen ist. So können entsprechend Medikamente und Produkte mit präventivem Charakter konsumiert werden mit dem Ziel, die Gesundheit zu erhalten oder zu verbessern oder aber, um einer Absenkung des Gesundheitskapitalstocks entgegenzuwirken und somit zumindest das Ausgangsniveau erneut zu erreichen.

Die Investitionen in die Gesundheit und hier insbesondere die Entscheidung über den Konsum von Gesundheitsleistungen hängen wesentlich vom verfügbaren Einkommen $Y_{v}$ ab. Dieses Einkommen umfasst zunächst das gesamte Arbeitseinkommen, bestehend aus dem kurzfristig konstanten Lohnsatz $w$, der dem Wertgrenzprodukt der Arbeit der Vorperiode entspricht, und der aktuellen Arbeitszeit $t_{w}$. Ein positives Arbeitseinkommen kann nur dann erzielt werden, wenn das Individuum über eine ausreichend gute Gesundheit $H>H_{k}$ verfügt, wobei $H_{k}$ einen exogen gegebenen kritischen Gesundheitskapitalstock abbildet. Wird dieser unterschritten, folgt hieraus Arbeitsunfähigkeit, d. h. die Arbeitszeit und folglich das Arbeitsangebot reduzieren sich (kurzfristig) auf Null.

Gegen hieraus resultierende Einkommensverluste ist das Individuum über eine lohnsteuerfinanzierte Krankenversicherung abgesichert, wobei $\tau$ den Steuersatz angibt. ${ }^{72}$ Diese Krankenversicherung garantiert zum einen ein festes Einkommen im Krankheitsfall, $\bar{Y}$, das unter dem letzten Nettoerwerbseinkommen liegt. ${ }^{73}$ Zum anderen werden finanzielle Aufwendungen, die aus den Gesundheitsinvestitionen $M$ resultieren, vollständig oder teilweise übernommen, um die finanzielle Belastung für einzelne Individuen abzudämpfen.

Beschreibt nun $L$ die Summe aller Zahlungen, die nach einem Krankheitsschaden für die vollkommene Genesung oder aufgrund bestimmter präventiver Maßnahmen geleistet werden müssen, und gibt $v$ denjenigen Anteil dieser Zahlungen an, der von der Krankenversicherung übernommen wird, so stehen dem Individuum sein um die Steuerzahlungen gemindertes Einkommen sowie die Zuwendungen $v L$ der Krankenversicherung als Budget zur Verfügung. Anzumerken ist an dieser Stelle, dass die Leistungen der Krankenversicherung exogen sind und daher auch unabhängig vom Einkommen erfolgen, während die Finanzierung durch das Individuum über den Steuersatz $\tau$ direkt an das Einkommen gebunden ist. Zudem wird angenommen, dass $0<v \leq 1$ gilt, so dass die Versicherungsleis-

\footnotetext{
72 Ebenso denkbar wăre eine Beitragsfinanzierung, die ohne Beitragsbemessungs- und Versicherungspflichtgrenze einer proportionalen Lohnsteuer gleichkăme, oder aber eine Finanzierung über eine einkommens- und risikounabhängige Prämie.

${ }^{73}$ Andernfalls bestünde der Anreiz, sich dem Arbeitsmarkt vollständig zu entziehen und die Leistungen der Krankenversicherung dauerhaft in Anspruch zu nehmen.
} 
tung die Behandlungskosten nicht übersteigen kann. Aus diesen Überlegungen ergibt sich für den Zustand ausreichender Gesundheit folgende Budgetrestriktion:

$$
Y_{v}=(1-\tau) t_{w} w+v L=M+C+S \quad \text { für } H>H_{k} .
$$

Auf der linken Seite der Gleichung (5.2) ist das verfügbare Einkommen $Y_{v}$ bestimmt, das sich aus der Summe aus dem Nettoerwerbseinkommen und den Versicherungsleistungen, dargestellt in der Mitte der Gleichung, errechnet. Auf der rechten Seite dieser Budgetrestriktion sind die möglichen Verwendungsformen des Einkommens aufgeführt. Neben der gesamten Nachfrage nach Gesundheitsleistungen $M$ kann das verfügbare Einkommen zusätzlich aufgeteilt werden auf die Verwendungszwecke gesundheitsneutraler Konsum $C$ sowie Ersparnisse $S .{ }^{74}$ Die Nachfrage nach Gesundheitsleistungen wird folglich durch die Versicherungsleistung sowie durch denjenigen Anteil bestimmt, den das Individuum von seinem Nettoeinkommen für die Gesundheitsinvestitionen aufzuwenden bereit ist:

$$
M=\eta(1-\tau) w t_{w}+v L \quad \text { für } H>H_{k} .
$$

$\eta$ beschreibt einen variablen Proportionalitätsfaktor mit $0 \leq \eta<1^{75}$. Dieser Faktor gibt Aufschluss darüber, welcher Anteil des verfügbaren Einkommens für Gesundheitsleistungen und welcher Anteil $(1-\eta)$ für Konsum und Ersparnis aufgewendet wird. Je näher $\eta$ an Eins liegt, desto mehr Einkommen wird in die Gesundheit investiert und desto weniger wird gesundheitsneutral konsumiert und gespart. Werden hingegen keine finanziellen Eigenmittel für die Gesundheit aufgewendet, reduziert sich die Nachfrage auf $M=v L$ und damit auf die Versicherungsleistung.

Ohne Berücksichtigung des Zeitaufwands liegt der optimale private Anteil in diesem Fall genau bei $(1-v) L$. Liegt das tatsächlich aufgewendete Einkommen darunter, werden nicht alle präventiven sowie kurativen Maßnahmen ergriffen, die für den Erhalt bzw. eine Verbesserung der Gesundheit notwendig sind. Ein

\footnotetext{
${ }^{74}$ Es wird zur Vereinfachung angenommen, dass trotz der Ersparnisbildung kein Vermögen vorhanden ist, da hier lediglich eine Periode betrachtet wird. In einer mehrperiodigen Analyse wäre der Vermögensfall zu integrieren.

${ }^{75}$ Für $\eta=1$ würden der sonstige Konsum sowie die Ersparnisse auf Null reduziert. Dieser Sonderfall bleibt aus der folgenden Analyse ausgeklammert, da angenommen wird, dass ein Teil des normalen Lebensunterhalts durch die Individuen selbst bestritten werden muss.
} 
bestehender Gesundheitsschaden $L$ wird folglich nicht vollständig kompensiert. $^{76}$

Für das verfügbare Einkommen im Krankheitsfall gilt, dass sich dieses aus dem garantierten Einkommen $\bar{Y}$ und den von der Krankenversicherung übernommenen Leistungen ergibt:

$$
Y_{v}=\bar{Y}+v L=M+C+S \quad \text { für } H \leq H_{k} .
$$

Für die Nachfrage nach Gesundheitsleistungen folgt im Krankheitsfall:

$$
M=\eta \bar{Y}+v L \quad \text { für } H \leq H_{k},
$$

so dass für neutralen Konsum und Ersparnisse im Fall der Erwerbsunfähigkeit finanzielle Mittel in Höhe von $(1-\eta) \bar{Y}$ zur Verfügung stehen. ${ }^{77}$

\subsubsection{Arbeit und Gesundheitsinvestitionen}

Im Folgenden wird zunächst angenommen, dass keinerlei Freizeit $\left(t_{F}\right)$ zur Verfügung steht, so dass letztlich nur zwei Zeitverwendungszwecke existieren. Ein Teil der Zeit wird für Erwerbszwecke aufgewendet, um das Erwerbseinkommen, das hier die einzige Einkommensquelle darstellt, zu erhöhen. Alternativ kann Zeit in die Gesundheit investiert werden mit dem Ziel, den individuellen Gesundheitszustand zu verbessern. Die Zeitrestriktion bei ausreichender Gesundheit und damit bei Erwerbsfähigkeit lautet

$$
T=t_{w}+t_{M} \quad \text { für } H>H_{k} \text {. }
$$

Bei Krankheit hingegen reicht die individuelle Gesundheit nicht aus, um der Erwerbstätigkeit nachzukommen, so dass sämtliche Zeit in die Gesundheit investiert wird:

$$
T=t_{M} \quad \text { für } H \leq H_{k} .
$$

\footnotetext{
${ }^{76}$ Von einer möglichen Nachfrage nach Gesundheitsleistungen, die über das medizinisch notwendige hinausgehen, wird an dieser Stelle abstrahiert.

${ }^{77}$ Der Krankheitsfall wird nachfolgend nicht weiter behandelt, da in diesem Fall die Arbeitszeit als relevante Entscheidungsvariable auf Null sinkt.
} 
Die Dauer einer Krankheit, die das kurzfristige Arbeitsangebot auf Null reduziert, hängt von der Investitionstätigkeit in diesem Zeitraum ab, da sich nur so eine Verbesserung der Gesundheit einstellt. Allgemein gilt, dass der kritische Gesundheitskapitalstock $H_{k}$ wieder überschritten werden muss.

Ein Absinken des Gesundheitskapitalstocks wird im vorliegenden Modell über die Abschreibungen $\delta$ erklärt:

$$
\delta=\delta_{u}+\delta_{w} \quad \text { mit } \delta^{\prime}=\frac{\partial \delta_{w}}{\partial t_{w}}>0, \frac{\partial^{2} \delta_{w}}{\partial t_{w}^{2}} \geq 0 .
$$

Die Abschreibungen setzen sich additiv aus arbeitszeitunabhängigen Abschreibungen $\left(\delta_{u}\right)$ sowie aus arbeitszeitabhängigen Abschreibungen $\left(\delta_{w}\right)$ zusammen. Es wird angenommen, dass eine hohe Arbeitszeit mit Stress und wenig Regeneration verbunden ist, was sich über die Abschreibungsrate negativ auf die Gesundheit auswirkt. ${ }^{78}$ Auch ohne Arbeit bleibt die Abschreibungsrate positiv, da sie durch weitere Faktoren determiniert wird, beispielsweise durch den natürlichen Alterungsprozess sowie durch eine ungesunde Lebensweise (vgl. u. a. Grossman (1972)). In diesem Fall wird angenommen, dass die Abschreibungsrate in Höhe von $\delta_{u}$ konstant ist. Andernfalls würde eine Reduktion der Arbeitszeit auf Null dazu führen, dass die Gesundheit auf gleich bleibendem Niveau verharrt. Tabelle 2 gibt noch einmal einen Überblick über die verwendeten Modellvariablen.

Für das Arbeitseinkommen ist neben dem Lohnsatz, der im Modell kurzfristig konstant ist, die Arbeitszeit entscheidend. Letztere beeinflusst die Gesundheit über drei verschiedene Faktoren. Zum einen determiniert die Arbeitszeit das Einkommen und damit den finanziellen Rahmen der Gesundheitsnachfrage, zum Zweiten limitiert Arbeit auch die zur Verfügung stehende Investitionszeit (vgl. Ettner (2000), S. 3). Drittens werden die Abschreibungen durch die Arbeitszeit

${ }^{78}$ Ebenso wäre es denkbar, dass Arbeit bis zu einem bestimmten Belastungsgrad nicht als Übel angesehen wird, so dass beispielsweise erhöhte körperliche oder geistige Anstrengung durch Anerkennung und Selbstverwirklichung überkompensiert werden kann. Hieraus würde sich ein positiver Einfluss der Arbeitszeit auf die Abschreibungsrate ergeben, der negative Einfluss der arbeitszeitunabhängigen Abschreibungen würde abgeschwächt. Damit würden die Abschreibungen nicht mehr monoton steigen, sondern es ergäbe sich vielmehr ein u-förmiger Verlauf, indem die Abschreibungen zunächst mit der Aufnahme einer Erwerbstätigkeit absinken und erst wieder ansteigen, wenn die Belastungen durch die Arbeit die positiven Effekte übersteigen. In diesem Fall müssten die nachfolgenden Ergebnisse gesondert betrachtet werden, wovon jedoch abgesehen wird. 
beeinflusst. Letztlich sind eine hohe Arbeitszeit und damit ein hohes Arbeitsangebot wiederum nur dann möglich, wenn die Gesundheit das kritische Niveau nicht unterschreitet. Aus diesem Trade-off ergibt sich das Arbeitsangebot als die für die Gesundheit relevante Entscheidungsvariable.

Tabelle 2: Überblick über die Modellvariablen

\begin{tabular}{|ll|}
\hline$H$ & Gesundheitskapitalstock \\
$H_{K}$ & Kritischer Gesundheitskapitalstock, bei dessen Unterschreiten \\
& Arbeitsunfähigkeit vorliegt \\
$\delta_{i}$ & Nachfrage nach Gesundheitsleistungen \\
$t_{M}$ & Abschreibungsrate \\
$t_{w}$ & Investitionszeit \\
$t_{F}$ & Frbeitszeit \\
$Y_{v}$ & verfügbares Einkommen bei ausreichender Gesundheit \\
$\tau$ & Steuersatz \\
$w$ & Lohnsatz \\
$v$ & Deckungssatz der Krankenversicherung \\
$L$ & Finanzieller Aufwand für eine vollständige Genesung \\
$C$ & gesundheitsneutraler Konsum \\
$S$ & Ersparnisse \\
$\bar{Y}$ & durch die Krankenversicherung garantiertes Einkommen bei Ar- \\
& beitsunfähigkeit \\
$\eta$ & Anteil des Einkommens, der für Gesundheitsinvestitionen auf- \\
$T$ & gewendet wird \\
\hline
\end{tabular}

Im Folgenden wird für den Fall ausreichender Gesundheit untersucht, welche Auswirkungen eine marginale Veränderung der Arbeitszeit auf die Gesundheit besitzt. In diesem Fall ist das Auftreten einer Krankheit zwar grundsätzlich möglich, der Gesundheitskapitalstock sinkt jedoch nicht unter die kritische Grenze, so dass das Individuum nach wie vor erwerbsfähig ist.

Differenzieren des in (5.1) definierten Gesundheitskapitalstocks nach $t_{w}$ unter Berücksichtigung von (5.3), (5.6) sowie von (5.8) führt zu 


$$
\frac{d H}{d t_{w}}=\eta(1-\tau) w H_{1}-\frac{\partial t_{M}}{\partial t_{w}} H_{2}+\delta_{w}^{\prime} H_{3} \quad \text { für } H>H_{k} \text {. }
$$

Der Einfluss der Arbeitszeitentscheidung auf die individuelle Gesundheit, dargestellt in Gleichung (5.9), gliedert sich in drei verschiedene Partialeffekte auf. Der erste Term auf der rechten Seite beschreibt den Einkommenseffekt einer Ausweitung der Arbeitszeit, indem die Veränderung der Gesundheit aufgrund einer veränderten Nachfrage nach Gesundheitsleistungen aufgezeigt wird. Der mittlere Term ist Ausdruck des Zeiteffekts, der sich aus einer Ausweitung der Arbeitszeit ergibt. Dieser Effekt gibt an, wie stark die Erträge aus der Investitionszeit aufgrund einer Arbeitszeiterhöhung sinken. Der rechte Term schließlich beschreibt die negative Wirkung der Arbeitszeit auf die Gesundheit über die Höhe der Abschreibungen, im Folgenden Abschreibungseffekt genannt.

Die genaue Wirkung des Einkommenseffekts $\left(\eta(1-\tau) w H_{1}\right)$ bei einer Ausweitung der Arbeitszeit erklärt sich wie folgt: Eine positive Veränderung der Arbeitszeit erhöht die Nachfrage nach Gesundheitsleistungen um das zusätzlich verfügbare Lohneinkommen, gewichtet mit dem Proportionalitätsfaktor $\eta$, der angibt, welcher Anteil des hinzugekommenen Einkommens in die Gesundheit investiert werden soll. Der Grenzertrag des zusätzlichen Konsums von Gesundheitsgütern ist positiv, so dass sich aus einer gesteigerten Nachfrage nach Gesundheitsleistungen eine Verbesserung der Gesundheit ergibt. Je höher der Konsum folglich ist, desto besser ist c. p. auch die Gesundheit.

Weiterhin werden abnehmende Grenzerträge der Gesundheitsgüter unterstellt. Je besser der individuelle Gesundheitszustand bereits ist, desto mehr gesundheitsfördernde Güter werden benötigt, um einen Anstieg im Gesundheitskapitalstock $\mathrm{zu}$ erzielen. Diese Ausweitung muss nicht ausschließlich quantitativer Natur sein, denn insbesondere eine Verbesserung der Qualität der nachgefragten Gesundheitsgüter kann zusätzlich investiertes Einkommen voraussetzen. ${ }^{79}$

Der zweite in Gleichung (5.9) enthaltene Term $\mathrm{H}_{2}$ ist Ausdruck des Zeiteffekts, der sich aus einer Ausweitung der Arbeitszeit ergibt. Die Grenzerträge der Investitionszeit sind positiv, während sich aus der vorangestellten partiellen Ableitung der Investitions- nach der Arbeitszeit die Stärke des Effekts ergibt. Da in diesem vereinfachten Modell nur zwei verschiedene Zeitverwendungszwecke

${ }^{79}$ Eine Ausweitung der nachgefragten Menge bezieht sich auf Gesundheitsgüter der ersten und zweiten Kategorie (Substitute bzw. Komplemente), während es sich bei Leistungen mit höherer Qualität um Güter der dritten Kategorie handelt (vgl. Kapitel 5.2). 
existieren, führt ein Anstieg der Arbeitszeit zu einem Rückgang der Investitionszeit in gleichem Umfang.

Im Hinblick auf den Grenzertrag der Investitionszeit sind zwei verschiedene Resultate denkbar. Werden die Grenzerträge zunächst als konstant positiv angenommen $\left(\partial H / \partial t_{M}>0, \partial^{2} H / \partial t_{M}{ }^{2}=0\right)$, ist auch der Zeiteffekt $H_{2}$ in (5.9) konstant, so dass das Gesundheitskapital mit zunehmender Arbeitszeit c. p. proportional absinken würde. Aufgrund der Annahme der sinkenden Grenzerträge der nachgefragten Gesundheitsleistungen kann aber nicht jede Zeiteinheit gleichermaßen durch eine Steigerung des Gesundheitsgüterkonsums ausgeglichen werden. Vielmehr muss die Nachfrage hiernach immer weiter ausgedehnt werden, um den Zeiteffekt zu kompensieren.

Weitaus realistischer hingegen erscheint das zweite mögliche Szenario der fallenden Grenzerträge der Zeitinvestitionen $\left(\partial H / \partial t_{M}>0, \partial^{2} H / \partial t_{M}{ }^{2}<0\right)$, denn durch diese Annahme sind Zeitaufwand und materielle Gesundheitsgüter nur noch begrenzt gegeneinander substituierbar. In diesem Fall muss die Nachfrage nach Gesundheitsleistungen mit zunehmender Arbeitszeit noch stärker ansteigen als im Fall konstanter Grenzerträge. Folglich muss auch der Anteil der privat finanzierten Gesundheitsleistungen $\eta$ mit einer höheren Rate ansteigen mit der Konsequenz, dass der Einkommensteil, der für privaten Konsum sowie Ersparnisse zur Verfügung steht, immer weiter absinkt.

Gleichzeitig impliziert ein hoher Arbeitszeitanteil einen geringen Aufwand an Investitionszeit. Je weniger Zeit für Investitionen jedoch zur Verfügung steht, desto stärker wirkt sich der Zeiteffekt aufgrund der Annahme der sinkenden Grenzerträge negativ auf die Gesundheit aus. Um diesem Effekt entgegenzuwirken, müssen vermehrt Gesundheitsleistungen konsumiert werden. Aufgrund der Annahme fallender Grenzerträge aus dem Konsum von Gesundheitsgütern muss der Investitionsanteil dann überproportional zum Einkommen ansteigen, so dass sich der Verlust an Investitionszeit mit zunehmender Arbeitszeit immer schwerer kompensieren lässt. Gleiches gilt für einen besonders geringen Arbeitszeitanteil und ein hiermit verbundenes niedrigeres Konsumniveau an Gesundheitsleistungen.

Aus diesen Überlegungen folgt, dass die einzelnen Investitionskomponenten im Hinblick auf den optimalen Gesundheitskapitalstock nur begrenzt substituierbar sind und dass die Komplementarität dieser Faktoren umso größer wird, je geringer eine der beiden Zeitkomponenten in ihrer Ausprägung ist.

Zusätzlich zu den Einkommens- und Zeiteffekten resultiert ein Abschreibungseffekt $\delta_{w}^{\prime} H_{3}$ aus einer Veränderung der Arbeitszeit. Während die Abschreibun- 
gen um so höher sind, je höher die gewählte Arbeitszeit ausfällt, $\delta_{w}$ also strikt positiv ist, ist der Einfluss der Abschreibungen auf das Gesundheitskapital $\mathrm{H}_{3}$ und damit auf die gesamte Gesundheitskapitalakkumulation negativ. Es wird in diesem Zusammenhang angenommen, dass eine hohe Arbeitszeit mit einer erhöhten körperlichen Anstrengung einhergeht, so dass hierdurch die Leistungsfähigkeit stetig abnimmt. Über die Effektivität der körperlichen Arbeit hinaus wird auch die geistige Produktivität negativ durch die Arbeitszeit beeinflusst, denn insbesondere Stress und Leistungsdruck führen dazu, dass die Arbeitszeit über die Abschreibungen einen negativen Einfluss auf die Gesundheit besitzt (vgl. Van Zon und Muysken (2001), S. 175). ${ }^{80}$

Die gesundheitsoptimale Arbeitszeit ist dann erreicht, wenn sich eine Veränderung der Arbeitszeit nicht weiter auf die Gesundheit auswirkt, d.h. wenn $\mathrm{d} H / \mathrm{d} t_{w}=0$. Anschließendes Auflösen von (5.9) ergibt

$$
\frac{\partial t_{M}}{\partial t_{w}}=-\frac{E E+A E}{Z E} .
$$

$E E$ ist Ausdruck des Einkommenseffekts, $Z E$ kennzeichnet den Zeiteffekt und $A E$ den Abschreibungseffekt. Unter Berücksichtigung der Tatsache, dass bei nur zwei Zeitverwendungszwecken

$$
\frac{\partial t_{M}}{\partial t_{w}}=-1
$$

ist, folgt aus (5.10)

$$
E E=Z E-A E
$$

Für den Einkommenseffekt gilt, dass dieser auch als Wertgrenzprodukt der Gesundheitsleistungen interpretiert werden kann: Je höher der Lohnsatz ist, desto höher ist auch das verfügbare Erwerbseinkommen nach Abzug der Steuerzahlungen und desto mehr Zeit kann durch die Inanspruchnahme von Gesundheitsleistungen substituiert werden. Daher wird die für die Gesundheit optimale Zeitallokation in Richtung Arbeitszeit verlagert, wodurch noch weiteres Einkommen erzielt werden kann (vgl. Grossman und Benham (1974), S. 215). Hierbei ist

\footnotetext{
${ }^{80}$ Leontaridi und Ward (2002) führen als Konsequenzen von andauerndem Stress Krankheit, eine geringe Produktivität sowie eine erhöhte Zahl an Arbeitsunfällen an.
} 
jedoch zu beachten, dass das Wertgrenzprodukt durch die abnehmenden Grenzerträge der Gesundheitsinvestitionen gekennzeichnet ist, so dass für eine Verbesserung der Gesundheit immer mehr Gesundheitsleistungen nachgefragt werden müssen. Gleichung (5.11) zeigt, dass die gesundheitsoptimale Arbeitszeit genau dann erreicht ist, wenn dieser positive Einkommenseffekt die negativen Einflüsse des Zeiteffekts sowie des Abschreibungseffekts kompensiert. Eine weitere Ausweitung der Arbeitszeit ermöglicht zwar einen gesteigerten Konsum an Gesundheitsleistungen $M$, der zusätzliche Ertrag hieraus reicht jedoch nicht aus, um die negativen Auswirkungen in Form einer höheren Abschreibungsrate sowie eines geringeren Zeiteffekts auszugleichen. ${ }^{81}$

\subsubsection{Freizeit als weitere Zeitverwendung}

Nun erscheint die Annahme von nur zwei Zeitverwendungszwecken sehr restriktiv. Eventuell gesundheitsschädigendes Verhalten ist im vorangegangenen Modellabschnitt zwar in den unabhängigen Abschreibungen $\delta_{u}$ enthalten, doch impliziert diese Vorgehensweise, dass hiermit keinerlei Zeitaufwand verbunden ist. Des Weiteren steht keine Zeit für reine Freizeitaktivitäten und damit für Konsum zur Verfügung. Im Folgenden wird daher Freizeit explizit als dritte Zeitverwendung in das Modell mit aufgenommen. Sie umfasst zum einen sämtlichen übrigen Zeitaufwand, der sich nicht im Sinne der Investitionszeit positiv auf die Gesundheit auswirkt, also als gesundheitsneutral oder sogar gesundheitsschädigend zu bezeichnen ist. Zum anderen sind hierin Freizeitaktivitäten enthalten, deren positive Auswirkungen auf die Gesundheit nicht bekannt sind oder in der Zeitallokationsentscheidung nicht näher berücksichtigt werden, so dass hier nicht von einem gesteuerten Investitionsprozess wie im Rahmen der Investitionszeit ausgegangen werden kann. Aktivitäten, bei denen eine Verbesserung der Gesundheit bewusst herbeigeführt wird oder bei denen zumindest bekannt ist, dass sich diese positiv auf die Gesundheit auswirken, werden im Rahmen der Investitionszeit berücksichtigt. Die insgesamt zur Verfügung stehende Zeit teilt sich entsprechend nicht mehr nur auf Arbeit und Gesundheitsinvestitionen auf, sondern umfasst zusätzlich noch die Möglichkeit, Freizeit $t_{F}$ in Anspruch zu nehmen:

${ }^{81}$ Weiterhin von Interesse für die optimale Zeitallokation ist der Einfluss exogener Größen wie des Steuersatzes auf die Arbeitszeit. Totales Differenzieren von Gleichung (5.9) nach der Arbeitszeit und dem Steuersatz führt zu keinem eindeutigen Ergebnis. Vielmehr ist der Einfluss des Steuersatzes einerseits davon abhängig, wie viel Zeit und medizinische Leistungen fur die Gesundheit aufgewendet werden, d. h. wie sich die Investitionen in die Gesundheit insgesamt zusammensetzen. Andererseits bestimmt auch der direkte Zusammenhang von Gesundheitsleistung $M$ und Investitionszeit $t_{M}$ die Wirkung, die davon abhăngt, ob es sich hierbei um Komplemente oder um Substitute handelt oder ob die Investitionskomponenten voneinander unabhängig sind. 


$$
T=t_{w}+t_{M}+t_{F} \quad \text { für } H>H_{k}
$$

beziehungsweise im Fall von Krankheit

$$
T=t_{M}+t_{F} \quad \text { für } H \leq H_{k} .
$$

Aufgrund der Tatsache, dass sich nun nicht mehr nur die Arbeitszeit negativ auf die Gesundheit auswirkt, sondern dass auch Freizeitaktivitäten gesundheitsschädigend sein können, wird die Freizeit explizit in die Definition der Abschreibungen mit aufgenommen. Damit verändert sich (5.8) zu

$$
\delta=\delta_{u}+\delta_{w}+\delta_{F},
$$

wobei $\delta_{F}$ die freizeitbedingten Abschreibungen kennzeichnet mit

$$
\delta_{F}^{\prime}=\frac{\partial \delta}{\partial t_{F}}>0, \frac{\partial^{2} \delta}{\partial t_{F}^{2}} \geq 0
$$

Die Abschreibungen sind hier additiv, da diese als voneinander unabhängig behandelt werden. Erneutes Differenzieren von (5.1) unter Berücksichtigung von (5.3) und der veränderten Bedingungen (5.12) und (5.14) ergibt nun

$$
\frac{d H}{d t_{w}}=\eta(1-\tau) w H_{1}+\frac{\partial t_{M}}{\partial t_{w}} H_{2}+\delta_{w}^{\prime} H_{3}+\frac{\partial t_{F}}{\partial t_{w}} \delta_{F}^{\prime} H_{3}=0 .
$$

Aus der veränderten Zeitrestriktion folgt weiterhin, dass

$$
\frac{\partial t_{F}}{\partial t_{w}}=-1-\frac{\partial t_{M}}{\partial t_{w}}
$$

so dass das Substitutionsverhältnis von Investitions- zu Arbeitszeit durch

$$
\frac{\partial t_{M}}{\partial t_{w}}=-\frac{E E+\left(\delta_{w}^{\prime} A E_{w}-\delta_{F}^{\prime} A E_{F}\right)}{Z E-\delta_{F}^{\prime} A E_{F}}
$$


beschrieben werden kann. $A E_{w}$ bezeichnet den Abschreibungseffekt, der sich aus der Erwerbstätigkeit ergibt, während $A E_{F}$ Abschreibungen aufgrund gesundheitsschädigenden Verhaltens in der Freizeit enthalten. Grundsätzlich gilt, dass eine Ausweitung der Arbeitszeit stets zu einem Rückgang der Investitionszeit führt, der jedoch nicht zwingend in gleicher Höhe ausfallen muss, da ein Teil der Zeit auch über einen Rückgang der Freizeit kompensiert werden kann. Ein hoher Einkommenseffekt bedeutet c. p. einen großen Einfluss der medizinischen Leistungen $M$ auf die Gesundheit, so dass bei einer Ausweitung der Arbeitszeit ohne Verluste in der Gesundheitsproduktion auf Investitionszeit verzichtet werden kann.

Der Einfluss der Abschreibungen ist jeweils negativ. Hohe arbeitszeitbedingte Abschreibungen müssen durch eine entsprechende Investitionszeit kompensiert werden, damit es nicht zu Gesundheitseinbußen kommt. In diesem Fall muss zur Erfüllung der Optimalitätsbedingung der Freizeitanteil sinken. Der Zeiteffekt im Nenner beschreibt hingegen den Einfluss der Investitionszeit auf die Gesundheit. Je wichtiger die Investitionszeit aufgrund der begrenzten Substituierbarkeit für die Akkumulation von Gesundheitskapital ist, desto größer ist dieser Zeiteffekt und desto weniger kann eine Ausweitung der Arbeitszeit über einen Rückgang der Investitionszeit ausgeglichen werden, so dass die gesteigerte Arbeitszeit zulasten der Freizeit geht.

Die bislang genannten Effekte wirken sich in direkter Weise auf die Relation zwischen Arbeitszeit und Investitionszeit aus. Darüber hinaus ist die Wirkung der Freizeit auf die Zeitallokation zu berücksichtigen, da diese die Gesundheit ebenfalls negativ beeinflussen kann $\left(\delta_{F}^{\prime}>0\right)$. Ein hoher gesundheitsschädigender Freizeitanteil führt zum einen dazu, dass sich die negativen Auswirkungen einer Arbeitszeiterhöhung verstärken. In diesem Fall müsste jedoch auch die Investitionszeit entsprechend hoch sein, damit sämtliche negativen Effekte der Freizeit ausgeglichen werden können. Auf der anderen Seite impliziert ein hoher Freizeitanteil c. p. geringere arbeitszeitbedingte Abschreibungen.

Wie groß der Einkommenseffekt nun im Verhältnis zu den Abschreibungen ist, wird im Wesentlichen durch den Proportionalitätsfaktor $\eta$ determiniert, da dieser angibt, welcher Teil des Nettoeinkommens für Gesundheitsleistungen aufgewendet werden soll. Die Höhe des Proportionalitätsfaktors hängt dabei maßgeblich von dem Erstattungssatz ab, mit dem die Krankenversicherung gesundheitsbedingte Ausgaben übernimmt. Damit sämtliche finanziellen Schäden $L$, die aus einer Minderung des Gesundheitskapitals resultieren, gedeckt sind, müssen die privaten Aufwendungen mindestens derjenigen Summe entsprechen, die notwendig ist, um den nicht von der Krankenversicherung übernommenen Teil zu finanzieren: 


$$
\eta(1-\tau) w t_{w} \geq(1-v) L
$$

und damit

$$
\eta \geq \frac{(1-v) L}{(1-\tau) w t_{w}}
$$

Aus (5.18) folgt für den Proportionalitätsfaktor, dass dieser zumindest genau so groß sein muss wie der Quotient aus den nicht von der Versicherung übernommenen Zahlungen und dem gesamten Nettoeinkommen, damit der Gesundheitszustand zumindest konstant gehalten werden kann. Dieser Quotient gibt entsprechend an, wie hoch der Anteil der Selbstbeteiligung am gesamten Nettoerwerbseinkommen ist. Je stärker der Proportionalitätsfaktor nun den Selbstbeteiligungsanteil übersteigt, desto mehr Gesundheitsleistungen werden zusätzlich zu einem bestehenden Schaden konsumiert und desto höher ist c. p. der Gesundheitskapitalbestand. Zudem ist der Eigenanteil $(1-v)$ und damit $\eta$ umso größer, je kleiner der Versicherungsanteil $v$ an dem insgesamt anfallenden finanziellen Schaden ist. Bei einem Anstieg der Versicherungsleistung werden bei konstantem Anteil $\eta$ entsprechend mehr Gesundheitsleistungen nachgefragt, eine konstante Nachfrage führt hingegen zu einem Absinken des privaten Anteils. Entsprechend müssen bei konstanter Nachfrage weniger finanzielle Mittel für die Gesundheitsinvestitionen aufgewendet werden, es kommt zu einer Umverteilung von Einkommen in Richtung privatem Konsum und Ersparnissen (vgl. Goodman et al. (1999), S. 802). Bei einer Vollfinanzierung von Gesundheitsschäden durch die Versicherung würden jedoch auch die Anreize, die Gesundheit durch präventive Maßnahmen zu erhalten, sinken, wodurch die Summe der für den Krankheitsschaden aufzuwendenden Zahlungen $L$ steigen würde. ${ }^{82}$ Aufgrund der Annahme, dass die Versicherungsleistungen nicht an das Einkommen angelehnt sind, wäre es für Individuen rational, mehr versicherte Leistungen nachzufragen als im Fall einer anteiligen Eigenfinanzierung. Da die Versicherungsleistung jedoch über die Steuereinnahmen finanziert werden muss, führt Vollversicherung zu einem Anstieg des Steuersatzes und somit in letzter Konsequenz zu einer Senkung des Nettoerwerbseinkommens.

Während der Steuersatz $\tau$ für den einzelnen exogen gegeben und damit ohne jegliche Entscheidungsalternative ist, können die Individuen die Höhe des Einkommensanteils $\eta$ selbst bestimmen. Hieraus ergibt sich die Frage, wann Indivi-

${ }^{82}$ Zum „Ex-ante Moral Hazard“" siehe Breyer et al. (2005), Kapitel 6.4f. 
duen einen hohen Deckungssatz präferieren bzw. wann ein hoher Eigenanteil gewünscht wird. Je geringer der durch die Krankenversicherung gedeckte Teil an dem auftretenden Schaden ist, desto mehr Gesundheitsleistungen müssen privat finanziert werden, vorausgesetzt die absolut nachgefragte Menge nach $\mathrm{Ge}$ sundheitsleistungen bleibt konstant. Aufgrund der Annahme, dass die Zahlungen von der Krankenversicherung einkommensunabhängig sind, würde sich aus einem Absinken des Deckungssatzes eine stärkere Belastung niedriger Einkommen ergeben, da hier der private Investitionsanteil $\eta$ stärker ansteigen müsste, um die bei konstanter Schadenshöhe $L$ entstehende zusätzliche Belastung zu finanzieren. Dieser Veränderung auf der Verwendungsseite des Einkommens steht eine Entlastung des verfügbaren Einkommens entgegen, denn aufgrund der Finanzierung der Versicherungsleistungen über die Steuereinnahmen kann bei einer Senkung des Deckungsanteils $v$ auch der Steuersatz $\tau$ gesenkt werden.

Übertragen auf eine Situation mit mehreren Individuen, die sich ausschließlich in ihrem Lohnsatz unterscheiden, werden höhere Einkommen aufgrund der Proportionalität der Steuerzahlungen zum Einkommen absolut stärker entlastet als niedrige Einkommen. Für die Individuen ergibt sich aus dieser Überlegung folgende Konsequenz: Wäre der Deckungssatz frei wählbar, würden diejenigen, deren erwartete Steuereinsparungen über den erwarteten Zusatzausgaben für Gesundheitsleistungen liegen, eine Senkung des Deckungssatzes befürworten, während Individuen mit geringem Einkommen einen hohen Deckungsanteil durch die Krankenversicherung vorziehen würden. Hieraus folgt, dass die Umverteilung von hohen zu niedrigen Einkommen umso größer ist, je mehr Krankheitskosten anteilig von der Krankenversicherung übernommen werden. Über die Modelldarstellung hinaus ist zudem zu berücksichtigen, dass Personen mit niedrigem Einkommen einem höheren Erkrankungsrisiko unterliegen und somit häufiger kurativen Gesundheitsausgaben ausgesetzt sind. Demzufolge steht dieser Gruppe weniger Einkommen für präventive Maßnahmen zur Verfügung. Damit ist eine zentrale Aufgabe der Krankenversicherung nicht nur in der reinen Risikoabdeckung zu sehen, sondern auch in der Umverteilung von hohen zu niedrigen Einkommen.

\subsubsection{Komparativ-statische Analyse}

Die bisherigen Ausführungen zeigen, dass die Arbeitszeit über den Einkommenseffekt einen positiven Einfluss auf die Gesundheit ausübt, während der Zeiteffekt sowie der Abschreibungseffekt zu einer Minderung des Gesundheitskapitals führen. Für die Gesundheit ergeben sich aus den geschilderten Zusammenhängen folgende Konsequenzen: Liegt die Arbeitszeit eines Individuums unter dem Optimum, so führt eine Ausweitung der Arbeitszeit zu einem höheren Einkommen und damit c. p. auch zu einer gesteigerten Nachfrage nach Gesundheitsleistungen. Es kommt zu einer positiven Veränderung des Gesundheitska- 
pitals. Wird die Arbeitszeit jedoch über das Optimum hinaus ausgeweitet, übersteigen die Verluste aus dem Zeiteffekt sowie aus den Abschreibungen den Einkommenseffekt. Dementsprechend ist es nicht mehr in ausreichendem Maße möglich, zeitintensive durch kapitalintensive Investitionen zu ersetzen, das Gesundheitskapital nimmt ab.

Abbildung 7 verdeutlicht diesen Zusammenhang. Auf der Abszisse ist die Arbeitszeit abgetragen, die Ordinate bildet den jeweiligen Gesundheitskapitalstock ab. Der konkave Verlauf der Gesundheitskapitalkurve $H(\eta)$ ergibt sich zum einen aus den abnehmenden Grenzerträgen der Investitionen in die Gesundheit, also der Verwendung von medizinischen Leistungen und Zeit, zum anderen aus dem zur Arbeitszeit konvexen Verlauf der Abschreibungen. Somit steigt die Gesundheit zunächst mit zunehmender Arbeitszeit an, nach Erreichen des Maximums fällt diese jedoch umso stärker wieder ab.

Abbildung 7: Gesundheit und Arbeitsangebot

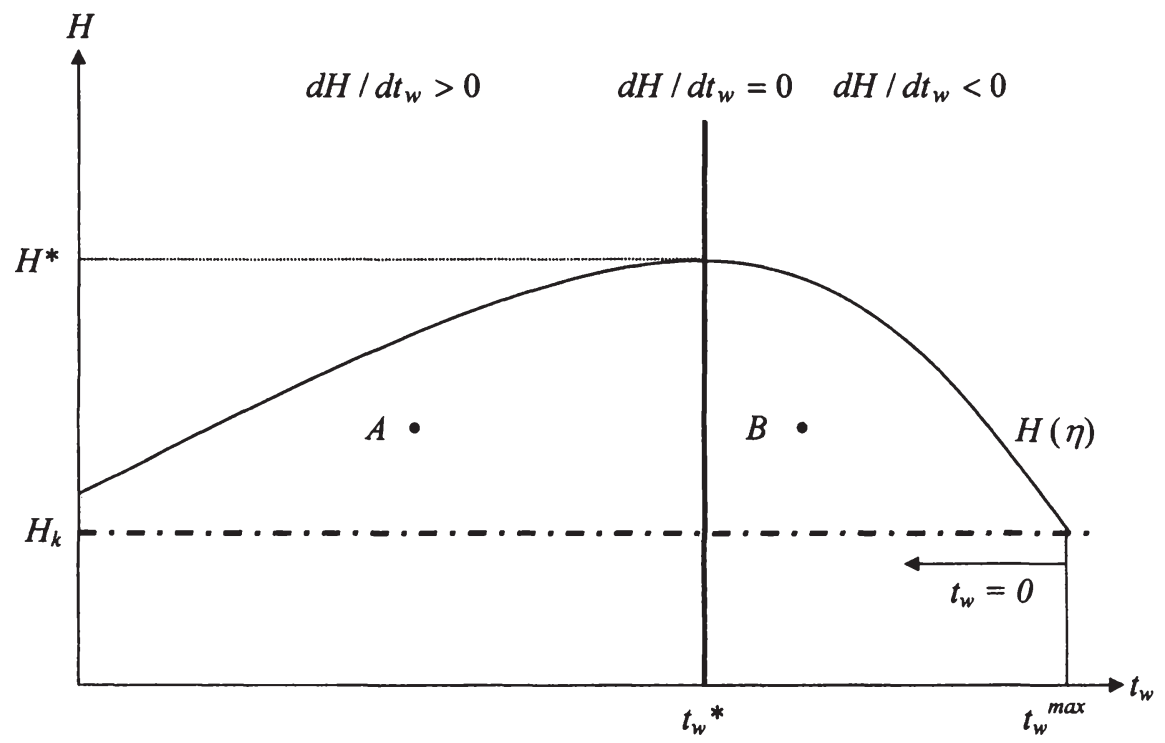

Quelle: Eigene Darstellung.

Bei einer Arbeitszeit von Null kann aufgrund des garantierten Einkommens $\bar{Y}$, das einen Basiskonsum an Gesundheitsleistungen garantiert, ein Gesundheitsniveau erzielt werden, das über dem kritischen Niveau liegt, so dass die Gesundheitskapitalkurve die Ordinate über dem kritischen Wert $H_{k}$ (dargestellt durch die Horizontale) schneidet. Der Abstand zwischen dem ohne Arbeit erzielbaren 
Gesundheitsniveau und der kritischen Grenze ist umso höher, je höher das bei Krankheit garantierte Einkommen ist. Ebenso ist es jedoch denkbar, dass das verfügbare Einkommen sowie die Leistungen aus der Krankenversicherung nicht ausreichen, um die für eine vollständige Genesung notwendigen Gesundheitsleistungen nachzufragen. In diesem Fall bliebe der erreichbare Gesundheitskapitalstock unterhalb der kritischen Grenze, es käme zu dauerhafter Erwerbsunfähigkeit.

Die Lage der Gesundheitskurve wird durch denjenigen Anteil des Einkommens $\eta$ bestimmt, der für die Gesundheit aufgewendet wird. Je höher dieser Anteil ist, desto höher ist auch die Lage der Gesundheitskapitalkurve $H(\eta)$ im Diagramm. Auf der anderen Seite ist bei der Wahl von $\eta$ zu beachten, dass hierdurch auch die Höhe des Konsums und der Ersparnisse determiniert wird. Die $H$-Kurve gibt die Gesundheit für einen gegebenen Wert $\eta$ an, bei dem das Individuum eine optimale Aufteilung der Ressourcen für die Verwendungszwecke Gesundheitsleistungen, sonstiger Konsum und Ersparnis erzielt. ${ }^{83}$

Beschreibt nun $\mathrm{d} H / \mathrm{d} t_{w}$ die Veränderungsrate der Gesundheit, dann ist die optimale Arbeitszeit $t_{w}{ }^{*}$ erreicht, wenn $\mathrm{d} H / \mathrm{d} t_{w}=0$ ist, da sich in diesem Punkt der Einkommenseffekt und der Zeit- und Abschreibungseffekt nivellieren und folglich das für ein konstantes $\eta$ maximale Niveau der Gesundheit $H^{*}$ erreicht ist. Liegt die Arbeitszeit $t_{w}$ links von diesem Gleichgewicht, ist die Veränderungsrate positiv $\left(\mathrm{d} H / \mathrm{d} t_{w}>0\right)$, so dass eine Ausweitung der Arbeitszeit c. p. eine Verbesserung der Gesundheit mit sich bringt. Eine Arbeitszeitallokation, die rechts des Gleichgewichts liegt, führt hingegen dazu, dass der Gesundheitskapitalbestand abnimmt $\left(\mathrm{d} H / \mathrm{d} t_{w}<0\right)$, da der Verlust an Investitionszeit und das Absinken der Gesundheit aufgrund steigender Abschreibungen nicht mehr über den Einkommenseffekt kompensiert werden können. Somit existiert ein Maximum an Arbeitszeit $t_{w}{ }^{\max }$, bei dem der minimale Gesundheitskapitalbestand $H_{k}$ erreicht wird. Bei Unterschreiten der kritischen Grenze reduziert sich die Arbeitszeit und damit das kurzfristige Arbeitsangebot auf Null. In diesem Fall sinkt das Einkommen auf den durch die Krankenversicherung garantierten Wert.

Die Fläche über diesem minimalen Gesundheitskapitalstock und unter der Gesundheitskapitalkurve gibt alle erreichbaren Kombinationen von Arbeitszeit und Gesundheit für einen konstanten Einkommensanteil $\eta$ an. Für sämtliche Punke auf der $H$-Kurve gilt, dass hier die optimale Investitionsmenge realisiert wird, d. h. eine Ausweitung der Arbeitszeit führt zu einer Bewegung auf der Kurve nach rechts. Für sämtliche Punkte unterhalb der Kurve und links des Gleichge-

${ }^{83}$ Um diesen optimalen Wert zu bestimmen, müsste die Nutzenfunktion des repräsentativen Individuums berücksichtigt werden. Hiervon wird weiterhin abgesehen. 
wichts gilt, dass das Gleichgewicht durch eine Veränderung der Zeitallokation erreicht werden kann.

Befindet sich ein Individuum im Punkt A, könnte die Gesundheitskapitalkurve bei gleicher Arbeitszeit erreicht werden, indem der Anteil der Investitionszeit im Verhältnis zur Freizeit erhöht würde, so dass bei konstantem Einkommenseffekt die negative Wirkung des Zeiteffekts sowie der aus der Freizeit entstehende Abschreibungseffekt gemindert würden. Ebenso könnte eine Bewegung hin zum gleichgewichtigen Gesundheitskapitalstock durch eine höhere Arbeitszeit erzielt werden, da hieraus ein gesteigertes Einkommen und damit ein höherer Einkommenseffekt resultieren würde.

Im Punkt B ist die Arbeitszeit im Hinblick auf das Gesundheitskapital hingegen zu hoch. Nun führen der Zeit- und der Abschreibungseffekt dazu, dass die Gesundheit im Laufe der Zeit immer weiter abnimmt, da die Veränderungsrate der Gesundheit in diesem Zustand negativ ist. Selbst für den Fall, dass die gesamte Restzeit ausschließlich für Gesundheitsinvestitionen aufgewendet würde, könnte die Kurve bei konstanter Arbeitszeit lediglich auf einem Punkt rechts vom Gleichgewicht erreicht werden, so dass kein Ausgleich der gegenläufigen Effekte mehr erzielbar wäre. Ebenso führte eine zusätzliche Ausweitung der Arbeitszeit bei konstantem $\eta$ ausschließlich weg vom Gleichgewicht, da die negativen Einflüsse auf die Gesundheit immer größer würden, während sich der positive Einkommenseffekt aufgrund der Annahme der sinkenden Grenzerträge immer weiter abschwächen würde. ${ }^{84}$

Für eine Erreichung des Gleichgewichts ist es daher unabdingbar, dass dem betreffenden Individuum bekannt ist, dass die für seine Gesundheit optimale Arbeitszeit bereits überschritten ist. Zudem muss eine Senkung der Arbeitszeit finanziell möglich sein, da diese eine gleichzeitige Senkung des gegenwärtigen Konsums impliziert. Dieser Effekt fällt umso stärker ins Gewicht, je niedriger die für Konsum verfügbare Zeit ist, da auch im Hinblick auf den Konsum Freizeit und Konsumgüter als nur teilweise substituierbar anzusehen sind. Einkommenserhöhungen aufgrund einer gestiegenen Arbeitsproduktivität, die aus einer verbesserten Gesundheit resultiert, lassen sich jedoch erst in der Folgeperiode realisieren, so dass die Bereitschaft, die Arbeitszeit zu senken und damit zunächst auf Konsum zu verzichten, maßgeblich von der Gegenwartspräferenz sowie den finanziellen Spielräumen abhängt. Zudem ist es möglich, dass die Ar-

${ }^{84}$ Leontaridi und Ward (2002) finden einen starken und signifikant positiven Effekt zu langer Arbeitszeiten auf den erfahrenen Stress und damit einen negativen Einfluss auf die Gesundheit. Zum negativen Einfluss zu hoher Arbeitszeiten auf die Gesundheit siehe daruber hinaus Shields (2000). 
beitszeit nicht ohne weiteres durch ein Individuum gesenkt werden kann. Liegt beispielsweise die vertraglich festgelegte Arbeitszeit über dem Optimum, ist ein Erreichen des Gleichgewichts durch das Individuum nicht mehr möglich.

Ein weiterer Grund für ein suboptimales Gesundheitsverhalten liegt in der mangelnden Substituierbarkeit von Arbeitskräften, die im Unternehmen Schlüsselpositionen bekleiden und folglich über unternehmensspezifisches Fachwissen verfügen, da in diesen Fällen Arbeit nicht ohne weiteres an andere Arbeitskräfte delegiert werden kann. Ebenso ist es möglich, dass die Fehlzeiten von Mitarbeitern negativen externen Effekten entsprechen, die dazu fuhren, dass das eigene Arbeitsangebot zu hoch wird, da die Arbeit des anderen ebenfalls übernommen werden muss. Die Folge hieraus sind häufig ein stetig zunehmendes Arbeitspensum und ein hoher Leistungsdruck, die zu permanenten Überstunden und damit zu einer schlechten Gesundheit der Betroffenen führen (vgl. Sparks et al. (1997), S. 391). ${ }^{85}$

\subsection{Konsequenzen für das Arbeitsangebot in der langen Frist}

Die in Kapitel 5.3 beschriebenen Zusammenhänge zwischen der Gesundheit und der durchschnittlichen Arbeitszeit werden im Folgenden hinsichtlich ihrer langfristigen Wirkung untersucht. So ist der Lohnsatz im Modell bislang aufgrund der kurzen Frist konstant gehalten. Unter der Annahme, dass die Produktivität eines Individuums maßgeblich durch die Gesundheit beeinflusst wird, folgt hieraus, dass ein guter Gesundheitszustand dazu führt, dass die Produktivität und damit auch der Lohnsatz der Folgeperiode ansteigt (vgl. Muurinen (1982), S. 14 und Sundberg (1996), S. 161), d. h. $w_{t}=w_{t}\left(H_{t-1}\right), \partial w_{t} / \partial H_{t-1}>0$ für den Fall $H_{t}$. ${ }_{1}>H_{K}$.

Für den Einfluss der Arbeitsangebotsentscheidung der Vorperiode $t_{w, t-1}$ auf den gegenwärtigen Lohnsatz $w_{t}$ bedeutet dies, dass letzterer genau dann ansteigt, wenn eine Ausweitung der Arbeitszeit in der Vorperiode zu einer Verbesserung der Gesundheit geführt hat:

$$
\frac{d w_{t}}{d t_{w, t-1}}=\frac{\partial w_{t}}{\partial H_{t-1}} \frac{\partial H_{t-1}}{\partial t_{w, t-1}}>0 \quad \text { fur } \frac{\partial H_{t-1}}{\partial t_{w, t-1}}>0 .
$$

${ }^{85}$ Häufig gehen Arbeitszeiten, die das individuelle Gleichgewicht überschreiten, neben zunehmendem Stress zudem mit gesundheitsschädigendem Verhalten wie Zigaretten- und Alkoholkonsum einher (vgl. Spurgeon et al. (1997), S. 370). Hieruber würde erneut die Abschreibungsrate erhöht. 
Insofern ist eine arbeitszeitbedingte Lohnsteigerung nur dann möglich, wenn die Gesundheit nicht optimal ist, da die rechte Seite ansonsten gleich Null ist. Bei einer optimalen Zeitallokation hingegen ist der Arbeitszeitanteil konstant, so dass sich hieraus keine Veränderungen für den Lohn mehr ergeben können. Im Optimum resultieren Lohnsteigerungen einzig aus den Produktivitätseffekten einer guten Gesundheit, nicht jedoch aus einer Veränderung der Arbeitszeit. Produktivitätssteigerungen lassen sich beispielsweise über einen gesteigerten finanziellen Investitionsanteil $\eta$ erreichen, da dieser die Gesundheit arbeitszeitunabhängig verbessert.

Die Steigerung des Lohnsatzes aufgrund einer Arbeitszeitausweitung in der vorangegangenen Periode bewirkt, dass der Einkommenseffekt bei nun gleich bleibendem Investitionsanteil ansteigt. Dementsprechend kann ein höherer finanzieller Betrag in die Gesundheit investiert werden mit dem Ziel, die Gesundheit und die Produktivität weiter zu steigern und sich somit langfristig ein höheres Einkommen zu sichern. Ein ähnliches Ergebnis findet sich bei Lee (1982), der einen positiven Einfluss des Lohnsatzes auf die Nachfrage nach Gesundheitsleistungen postuliert, so dass der Lohnsatz auch die Nachfrage nach Gesundheit steigert.

Gleichzeitig führt ein höheres Einkommen dazu, dass mehr finanzielle Mittel für Konsum und Ersparnisse bereitstehen. Hingegen hat ein gestiegener Lohnsatz auf die optimale Zeitallokation keinerlei Auswirkungen, wenn sich der für Gesundheitsleistungen aufgewendete Anteil am Einkommen $\eta$ adäquat verringert, da dann der Einkommenseffekt unverändert bleibt (vgl. Gleichung (5.16)). Bleibt dieser Anteil jedoch auch bei gestiegenen Löhnen nahezu gleich, führt ein höheres Einkommen bei konstanter Zeitallokation $\mathrm{zu}$ einer insgesamt verbesserten Gesundheit.

Gleichung (5.19) entsprechend führt ein Anstieg an krank verbrachten Tagen und damit ein Absinken des Arbeitsangebots zu einem Absinken des Lohnsatzes (vgl. Duraisamy und Sathiyavan (1998), S. 73 sowie Perri (1984), S. 211), da sich erstens aus der Gesundheit keine positiven Produktivitätseffekte mehr ergeben und da zweitens die Fähigkeit abnimmt, die Arbeitsaufgaben mit der gleichen Produktivität wie in den Vorperioden durchzuführen (vgl. Berkowitz et al. (1983), S. 145).

Liegt das von der Krankenversicherung garantierte Einkommen bei Abwesenheit in Höhe des Erwerbseinkommens oder nur unwesentlich darunter und sind die Konsequenzen von Fehlzeiten für den zukünftigen Lohn unbekannt, sinkt der Anreiz, Arbeit in vollem Umfang anzubieten, indem das individuelle Einkommen kurzfristig nicht oder nur kaum beeinflusst wird. Folglich kommt es zu 
Unterinvestitionen in das Humankapital. Ein ähnlicher Effekt ist einer steuerfinanzierten Krankenversicherung zuzuschreiben, da die Einkommenselastizität der Arbeitszeit kleiner Eins ist: Steigt der Lohnsatz, steigt das Bruttoeinkommen bei gleicher Arbeitszeit zwar an, das verfügbare Einkommen erhöht sich aufgrund der zu leistenden Steuerzahlungen jedoch nicht in gleichem Umfang, so dass sich die Lohnerhöhung nicht vollständig auf die private Nachfrage nach Gesundheitsleistungen überträgt. Somit kann die Arbeitszeit für einen gegebenen Gesundheitskapitalstock je nach Höhe der Steuerzahlungen nicht entsprechend gesenkt werden, falls sich der gewünschte Einkommenseffekt an der Bruttolohnerhöhung ausrichtet. Ebenso ist es denkbar, dass mehr Zeit in Arbeit investiert wird, damit die Gesundheitsnachfrage proportional zu der Lohnerhöhung steigen kann. Dies ist jedoch nur solange möglich, wie die tatsächliche Arbeitszeit noch unter dem Optimum liegt. Andernfalls würden die negativen Auswirkungen von Zeit- und Abschreibungseffekt überwiegen. ${ }^{86}$

Der Zusammenhang zwischen Arbeitszeit und Gesundheit bei variablem Lohnsatz ist in Abbildung 8 dargestellt. Auf der Abszisse wird erneut die Arbeitszeit abgetragen, auf der Ordinate der Gesundheitskapitalstock. Je höher der Lohnsatz c. p. ist, desto mehr Gesundheitsleistungen können absolut konsumiert werden unter der Prämisse, dass der relative Anteil der Gesundheitsleistungen am Einkommen $\eta$ konstant bleibt. ${ }^{87}$ Die Gesundheitskapitalkurve verschiebt sich nach oben. Aus dem höheren Einkommen folgt zum einen, dass bei gleicher Arbeitszeit eine höhere Nachfrage nach Gesundheitsleistungen erzielt werden kann mit dem Ergebnis, dass auch der Gesundheitskapitalstock ansteigt. Zum anderen wird die optimale Gesundheit $H^{* *}$ erst bei einer höheren Arbeitszeit $t_{w}^{\prime}$ " erreicht, da die negativen Wirkungen der Arbeit länger über den Einkommenseffekt kompensiert werden können.

$\mathrm{Da}$ die Arbeitszeit bei Individuen mit schlechter Gesundheit c. p. geringer ist als bei Individuen mit guter Gesundheit, führt der Einkommenseffekt dazu, dass ein Anstieg im Lohnsatz das Arbeitsangebot bei weniger Gesunden stärker ansteigen lässt als das Arbeitsangebot von Individuen mit guter Gesundheit (vgl. Lambrinos (1981), S. 209). Analog zu dem Fall des konstanten Lohnsatzes führt eine dauerhafte Ausweitung der Arbeitszeit über das Optimum hinaus zu einem Absinken des Gesundheitskapitalbestandes, indem der Einkommenseffekt den negativen Zeiteffekt nicht weiter kompensieren kann. Dies führt zu einem stetigen Absinken des Gesundheitskapitalbestandes bis auf den kritischen Wert, der

\footnotetext{
${ }^{86}$ Eine Analyse des Zusammenhangs zwischen Krankenversicherung und dem individuellen Arbeitsangebot findet sich in Currie und Madrian (1999).

${ }^{87}$ Würde dieser Einkommensanteil hingegen entsprechend sinken, würde sich kein positiver Einkommenseffekt aus einer Erhöhung des Lohnsatzes ergeben.
} 
die Arbeitszeit und damit das kurzfristige Arbeitsangebot auf Null absenkt. Das verfügbare Einkommen sinkt wiederum auf das von den Krankenversicherungen garantierte Niveau, das um so höher ist, je höher das Erwerbseinkommen ist, wenn das im Krankheitsfall garantierte Einkommen $\bar{Y}$ an das individuelle Einkommen angelehnt ist. Wird die Arbeitszeit über das Optimum hinaus ausgewietet, ergibt sich hieraus ein negativer Einfluss auf das Einkommen sämtlicher zukünftiger Perioden, wenn der Lohnsatz direkt an die Produktivität gekoppelt ist, da keine Produktivitätssteigerungen und damit keine Lohnzuwächse erzielt werden können. Schlechte Gesundheit führt folglich zu einem Verzicht auf ein höheres Lebenseinkommen in späteren Perioden.

Abbildung 8: Gesundheit und Arbeitszeit bei variablem Lohnsatz

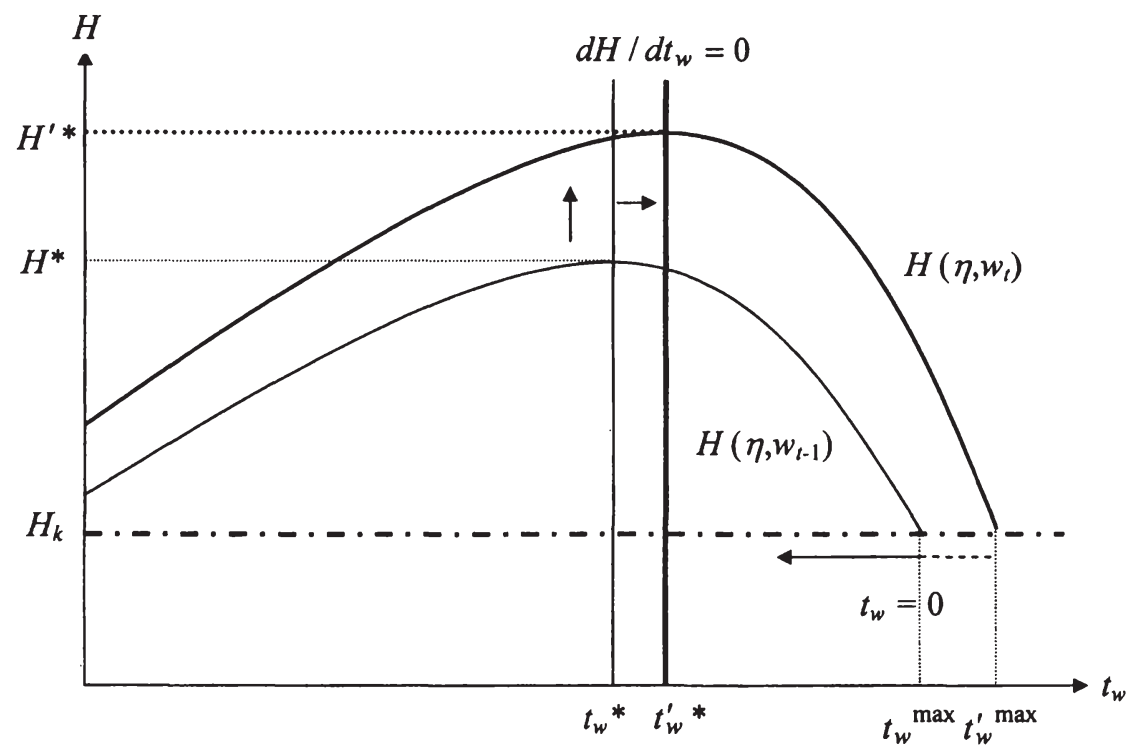

Quelle: Eigene Darstellung.

Neben dem aktuellen Lohnsatz ist die Lage der Gesundheitskapitalkurve auch von der Höhe sonstiger Einkunftsarten abhängig, von denen im Modell abstrahiert wird. Je höher der Anteil des Erwerbseinkommens am Gesamteinkommen ist, desto bedeutender ist der Einfluss der Arbeitszeit auf die Gesundheit, da hierüber die Stärke des Einkommenseffekts bestimmt wird. Ebenso ist es denkbar, dass ein niedriger Anteil des Erwerbseinkommens am insgesamt verfügbaren Einkommen dazu führt, dass die Abschreibungsrate niedriger ausfällt, da weniger Leistungsdruck vorhanden ist. Zudem sind insbesondere bei Arbeits- 
zeiten links des Optimums auch positive Abschreibungseffekte denkbar, indem nicht nur aus einer reinen Einkommensmotivation heraus gearbeitet wird, sondern sich direkte Nutzeneffekte aus der Arbeit ableiten lassen.

Weiterhin kann sich die Lage der kritischen Grenze $H_{k}$ im Zeitablauf verändern, da das Gefühl von Arbeitsunfahigkeit häufig subjektiver Natur ist. ${ }^{88}$ Anzumerken sei an dieser Stelle, dass bei einem hohen Arbeitszeitanteil umso mehr restliche Zeit für Investitionen aufgewendet werden muss, um den negativen Zeiteffekt zu verringern und ein Absinken des Gesundheitskapitals zu verlangsamen. Dementsprechend sinkt die für Freizeit und damit für übrigen Konsum verfügbare Zeit.

Unter der Annahme, dass Arbeitsproduktivität nicht ausschließlich von der Gesundheit abhängt, sondern auch von der Bildung, folgt hieraus der Schluss, dass weniger Zeit für Weiterbildung aufgewendet werden kann mit der Konsequenz, dass auch hier spätere Lohnzuwächse nicht in voller Höhe realisiert werden. Bei vollständigem Arbeitsausfall würde dieser Effekt noch verstärkt werden, da Prozessabläufe nicht weiter trainiert und die Effektivität der Produktion nicht weiter gesteigert werden können.

Mincer (1984) führt darüber hinaus an, dass nicht nur die Gesundheit Abschreibungen unterliegt, sondern dass auch Wissen und Bildung mit der Zeit an Wert verlieren und Fähigkeiten obsolet werden, so dass Erhaltungsmaßnahmen über die gesamte Lebenszeit durchgeführt werden müssen. Aus diesen Überlegungen folgt, dass sich die Opportunitätskosten einer schlechten Gesundheit nicht nur in den gegenwärtig entgangenen Lohneinbußen äußern, sondern dass darüber hinaus auch entgangene Lohnzuwächse in der Zukunft berücksichtigt werden müssen. Diese finanziellen Ressourcen stünden für erneute Investitionen in die Gesundheit zur Verfügung, woraus sich wiederum Produktivitätszuwächse ergeben würden. Unter der Annahme, dass die Grenzerträge der Gesundheitsleistungen zudem um so größer sind, je höher der Bildungskapitalstock eines Individuums ist, können besser gebildete Individuen leichter Zeit gegen Gesundheitsleistungen substituieren (vgl. Kemna (1987), S. 193) und damit ihr Arbeitsangebot ohne negative Konsequenzen für den Gesundheitskapitalstock ausweiten.

Aus diesen Überlegungen folgt, dass die Wahrscheinlichkeit einer längeren Erkrankung umso kleiner ist, je dichter die durchschnittliche Arbeitszeit an der optimalen Zeitallokation liegt. Eine konstant gute Gesundheit fuhrt im Zeitab-

${ }^{88}$ Objektive Arbeitsunfähigkeit ist insbesondere bei einem körperlichen Zustand gegeben, bei dem die berufliche Tătigkeit faktisch nicht weiter ausgeübt werden kann, und zwar unabhängig von der Arbeitsbereitschaft der einzelnen Personen. 
lauf zu einem quantitativ höheren Arbeitsangebot. Des Weiteren führen die genannten Zusammenhänge aufgrund einer gesteigerten Produktivität zu einer qualitativen Verbesserung der Arbeitsleistung und damit zu Outputsteigerungen. Die hier angeführten Überlegungen legen den Schluss nahe, dass eine gute Gesundheit ein hohes Einkommen bedingt, während schlechte Gesundheit dazu führt, dass das längerfristige Einkommen auf niedrigem Niveau stagniert.

\subsection{Zusammenfassung und Ausblick}

Die Gesundheit eines Individuums beeinflusst nicht nur seine gegenwärtige Arbeitsangebotsentscheidung und damit sein aktuelles Einkommen, sondern auch den Lohnsatz und damit das Einkommen zukünftiger Perioden. Eine gute Gesundheit führt zudem nicht nur zu direkten Produktivitätssteigerungen in den täglichen Arbeitsprozessen, sondern auch in der Akkumulation von Bildungskapital als zweite wesentliche Komponente des Humankapitals. Folglich können frühe Investitionen in die Gesundheit von Kindern, beispielsweise über Impfungen oder über eine gesunde Ernährung, das gesamte Lebenseinkommen positiv beeinflussen (vgl. Schultz (1997), S. 147).

Aufgrund des positiven Zusammenhangs zwischen der nachgefragten Menge an Gesundheitsleistungen und dem Einkommen sind derartige Investitionen in die eigene Gesundheit sowie in die Gesundheit von Kindern bei Individuen mit geringem Lohnsatz jedoch nur dann möglich, wenn die Arbeitszeit entsprechend hoch ist. Je höher jedoch der individuelle Arbeitszeitanteil an der insgesamt verfügbaren Zeit ist, desto weniger Investitionszeit steht zur Verfügung und desto schwerer werden Zeit und Gesundheitsleistungen gegeneinander substituierbar. Folglich können Gesundheitsleistungen nicht in ausreichender Höhe nachgefragt werden, so dass die Gesundheitsinvestitionen in ihrer Summe zu niedrig sind. Dementsprechend führt ein zu geringer Lohnsatz zu Arbeitszeiten, die über der gesundheitsoptimalen Arbeitszeit liegen und eine Verbesserung der Gesundheit unmöglich machen. Strauss und Thomas (1998) weisen zudem darauf hin, dass insbesondere bei gering entlohnter Arbeit die körperliche Verfassung eine besonders große Rolle spielt. Dementsprechend ist Arbeit in niedrigeren Einkommensschichten häufig gesundheitsintensiv, woraus sich wiederum negative Konsequenzen infolge einer höheren Abschreibungsrate ergeben. Aufgrund der ausbleibenden Produktivitätseffekte werden im Zeitablauf keine Lohnsteigerungen erzielt, die Arbeitszeit kann nicht gesenkt werden. Dieser Kreislauf führt dazu, dass die Gesundheit derjenigen mit hohem Einkommen zu Produktivitätssteigerungen in der gesamten Volkswirtschaft führt, während gesundheitsbedingte Produktivitätszuwächse bei Gruppen mit geringem Einkommen kaum erzielt werden. 
Ist die Produktion innerhalb einer Volkswirtschaft arbeitsintensiv, so limitiert die Gesundheit die Produktionsmenge in direkter Weise aufgrund von Fehlzeiten und damit die Möglichkeit, die für das Wirtschaftswachstum ausschlaggebende Kapitalintensität zu erhöhen, da der Output sinkt und die Kapitalauslastung damit suboptimal wird. Eine höhere Produktivität der Beschäftigten führt hingegen dazu, dass der erreichbare Output bei gleichem Arbeitseinsatz ansteigt. Bei einer kapitalintensiven Produktion ist der direkte Einfluss der Gesundheit über die Arbeitskraft nicht in dem Ausmaß gegeben. Stattdessen werden Ressourcen für die Behandlung von Krankheiten benötigt, so dass dieses Kapital alternativen Verwendungszwecken entzogen wird. Weiterhin wirkt sich ein aus einem $\mathrm{zu}$ geringen Gesundheitskapitalstock resultierendes zu geringes Bildungsniveau negativ auf die Arbeitsproduktivität in allen Sektoren aus. Insbesondere betroffen ist der Forschungs- und Entwicklungssektor mit der Konsequenz, dass die Innovationstätigkeit sinkt, was sich letztlich negativ auf das Wirtschaftswachstum auswirkt.

Es kann gezeigt werden, dass Gesundheit zwar Voraussetzung für Wachstum ist, indem hierüber die Erwerbstätigkeit sowohl qualitativ als auch quantitativ beeinflusst wird. Auf der anderen Seite ist es aber gerade das Arbeitsangebot, das das Einkommen determiniert und so Investitionen in die Gesundheit ermöglicht. Somit handelt es sich hierbei um Faktoren, die einander bedingen: Ein schlechter durchschnittlicher Gesundheitszustand bremst die Einkommensentwicklung und damit das Wachstum, ohne Wachstum sind aber auch die Gesundheitsinvestitionen begrenzt. Ohne eine Initialzündung in Form eines exogenen Schocks kann diese Problematik nicht gelöst werden, das Einkommen bleibt auf niedrigem Niveau.

Ist die optimale Zeitallokation erreicht, ergeben sich Verbesserungen in der Gesundheit ausschließlich über steigende Einkommen, indem so die Nachfrage nach Gesundheitsgütern ansteigt. Tatsächlich ist die Gesundheit definitorisch jedoch nach oben begrenzt, indem ein theoretisch optimaler Gesundheitszustand existiert. Ist dieser Optimalzustand erreicht, können sich aus der Gesundheit keine weiteren Produktivitätseffekte mehr ergeben. Somit sind auch die Einkommenssteigerungen nach oben begrenzt, weshalb Gesundheit zwar Wachstum fördern kann, selbst aber im Gegensatz zu Bildung keinen elementaren Wachstumsmotor darstellt. Somit bleibt nachfolgend zu klären, von welchen Faktoren die Akkumulation von Bildungskapital abhängt und welche Bedeutung hier der Gesundheit zukommt. 


\section{Grundlegende Zusammenhänge zwischen Gesundheit und Bil- dung}

\subsection{Einleitung}

Die bisherigen Ausführungen konzentrieren sich im Wesentlichen auf die $\mathrm{Be}$ deutung der Bildung und der Gesundheit für das Wirtschaftswachstum, beschäftigen sich also unter anderem mit der Wirkung, die von einem hohen Bildungskapitalbestand ausgeht. Damit sich der Bildungskapitalbestand einer Ökonomie erhöht, müssen jedoch zuvor Investitionen in die Bildung getätigt werden. Diese hängen nicht zuletzt von der Einkommenssituation der Individuen ab, da Bildung stets mit Verzicht auf Erwerbstätigkeit und damit auf heutiges Einkommen einhergeht. Auf der anderen Seite spielen erwartete zukünftige Erträge eine Rolle, da diese Aufschluss über die Rendite der Investitionen geben.

Mit der Frage, wie Bildung und Einkommen miteinander zusammenhängen, beschäftigen sich Goetz und Hu (1996) sowie Bils und Klenow (2000). Beide Arbeiten kommen zu dem Ergebnis, dass Regionen mit höherem Bildungskapitalbestand höhere Wachstumsraten des Einkommens zu verzeichnen haben. Auf der anderen Seite begünstigt schnelleres Wachstum höhere Investitionen in das Bildungskapital, indem erstens die Diskontierungsrate mit steigendem Einkommen sinkt, wodurch zukünftige Erwerbseinkommen stärker gewichtet werden. Zweitens geht Wirtschaftswachstum mit Lohnanstiegen einher, so dass die ökonomische Attraktivität von Bildungsinvestitionen gesteigert wird.

Das Einkommen wirkt sich jedoch nicht nur positiv auf die Akkumulation von Bildungskapital aus, indem hierüber die individuelle Bildungsentscheidung beeinflusst wird, sondern auch, da die Rahmenbedingungen für Bildung verbessert werden. Zudem wird das individuelle Bildungsniveau maßgeblich durch die Bildung der Eltern determiniert, indem diese ihr Wissen direkt an die Kinder weitergeben können, die Kinder darüber hinaus aber auch unbewusst von den Eltern lernen, so dass man hier von externen Effekten sprechen kann. Auch gesamtwirtschaftlich ergeben sich externe Effekte, denn die Bildung der Eltern trägt zum durchschnittlichen Bildungsniveau in der Gesellschaft bei und fördert so den technischen Fortschritt in der Folgeperiode sowie die Adaption neuen Wissens. Durch diesen Fortschritt steigen die zukünftigen Erträge von Bildungsinvestitionen, so dass sich für die Kinder wiederum Bildungsanreize ergeben (vgl. Galor und Tsiddon (1997), S. 93f.). ${ }^{89}$

${ }^{89} \mathrm{Um}$ Wirtschaftswachstum zu generieren, kann es somit aus politökonomischer Sicht sinnvoll sein, zunächst nur Investitionen in Sektoren mit hoch qualifizierten Arbeitskräften zu 
Diese Ansätze wirken maßgeblich durch den positiven Einfluss des Einkommens auf die Bildung, so dass sich hieraus letztlich eine Aufwärtsspirale ergeben kann. Unter der Voraussetzung, dass Einkommen notwendig ist, um Bildung zu finanzieren, stellt sich hier die Frage, welche Maßnahmen getroffen werden müssen, um ärmeren Nationen den „Take-off" (Rostow (1990)) zu ermöglichen und nachhaltiges Wachstum zu generieren. Abbildung 9 zeigt mögliche Determinanten der Bildungsinvestitionen, aufgeteilt in Entwicklungsländer auf der linken und Industrienationen auf der rechten Seite. ${ }^{90}$

Abbildung 9: Determinanten der Bildungsinvestitionen

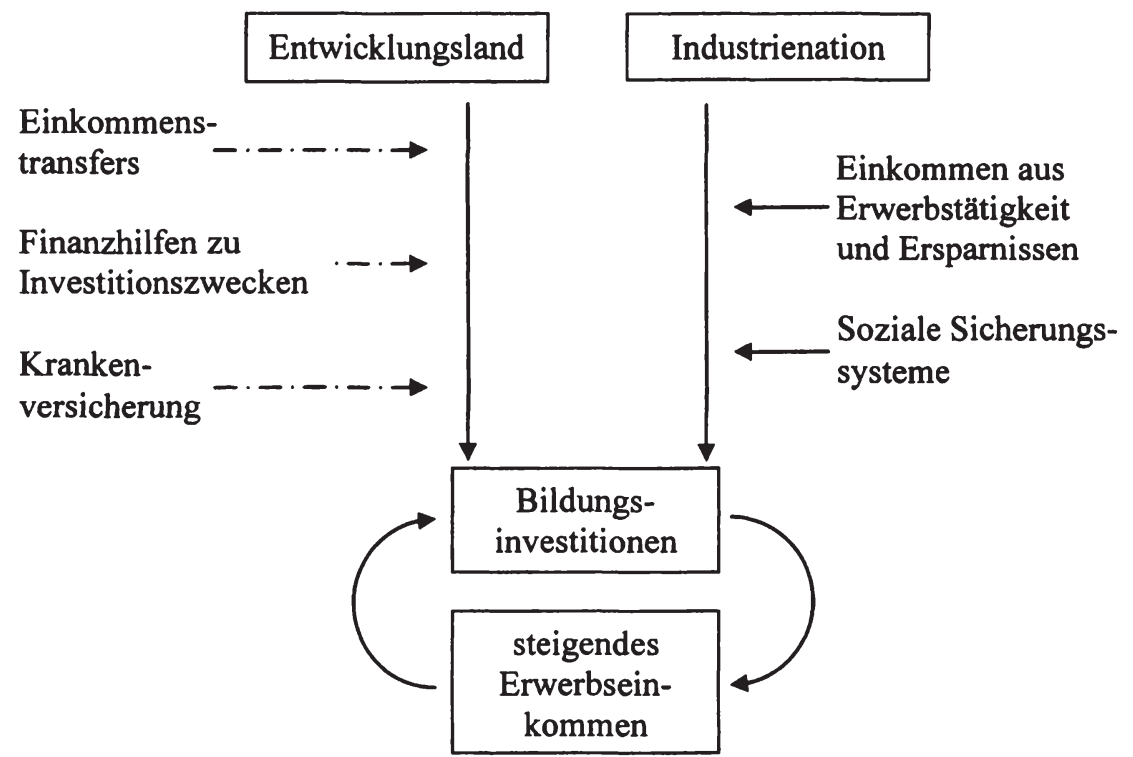

Quelle: Eigene Darstellung.

fordern und so Divergenz der Einkommen innerhalb eines Landes zuzulassen. Zwar steigt das Einkommen zunächst lediglich in dem betreffenden Sektor, über Technologiediffusion können sich hieraus jedoch auch positive Erträge in anderen Sektoren ergeben, so dass sich Bildungsinvestitionen für sämtliche Arbeitskräfte lohnen. Somit könnte es sinnvoll sein, bestimmte Gruppen in ihren Bildungsbemuhungen zu subventionieren, damit deren Externalităten Anreize für alle übrigen Gruppen liefern. Es ergibt sich folglich ein Trade-off zwischen kurz- und langfristiger Gleichheit und Stagnation auf der einen Seite sowie Ungleichheit in der kurzen Frist, gefolgt von Gleichheit und Wohlstand in der langen Frist.

${ }^{90}$ Es wird lediglich auf die Einkommenssituation der Haushalte abgestellt, übrige Ursachen für Unterschiede bleiben unberücksichtigt. 
Das Einkommen von Haushalten in Industrienationen wird im Wesentlichen durch das Erwerbseinkommen sowie durch Ersparnisse determiniert. Bei Erwerbsunfähigkeit durch Krankheit oder Alter sowie bei Arbeitslosigkeit gewährleisten die sozialen Sicherungssysteme zumindest ein Minimaleinkommen. Das Vorsorgesparen dient in diesem Zusammenhang hauptsächlich der Sicherung eines bestimmten Lebensstandards. Ist die finanzielle Absicherung gegen Arbeitsmarktrisiken gewährleistet, sinkt die Notwendigkeit, in jungen Jahren auf Bildung zu verzichten und stattdessen die Arbeitskraft auf dem Arbeitsmarkt anzubieten.

Anders stellt sich die Situation in Entwicklungsländern dar, da hier die Absicherung häufig innerhalb der Familie geschieht. Die Überlegung, in die Schulbildung $\mathrm{zu}$ investieren oder Investitionen $\mathrm{zu}$ unterlassen, hängt von folgenden Faktoren ab: Die ökonomische Motivation begründet sich ebenfalls in der erwarteten Rendite, denn das zukünftige Einkommen entspricht einer Kompensationszahlung für den während der Ausbildung erlittenen Einkommensverzicht. Darüber hinaus hängt die Bildungsentscheidung insbesondere in Entwicklungsländern davon $a b$, ob die Arbeitskraft während der Schulzeit für das Haushaltseinkommen entbehrlich ist. Wird das tägliche Leben aus diesem zusätzlichen Einkommen bestritten, ist Schulbildung nicht ohne weiteres finanzierbar. Schließlich spielen drittens die Erwartungen über mögliche Einkommensausfälle in der Zukunft, z. B. aufgrund von Krankheit, eine entscheidende Rolle. Einkommenstransfers können dazu beitragen, kurzfristige Einkommensverluste für die Dauer der Schulbildung auszugleichen und so die Familien finanziell entlasten. Gleiches gilt für die nachfolgende Erwerbsperiode, da nur so die Notwendigkeit des Vorsorgesparens verringert werden kann. Direkte Finanzhilfen zu Investitionszwecken können darüber hinaus dazu beitragen, die benötigte Infrastruktur bereitzustellen oder zu verbessern, um die Bildungsproduktivität zu steigern.

Abbildung 10 zeigt den Zusammenhang zwischen den Erträgen der Bildung in Form von zusätzlichem Erwerbseinkommen auf der einen und den hiermit verbundenen Kosten auf der anderen Seite. Zum Zweck einer vereinfachten Darstellung wird angenommen, dass nur zwei Einkommensprofile existieren: Entweder, es findet direkt nach Beendigung der Schulzeit im Alter von 15 Jahren ein Wechsel in das Berufsleben statt, oder aber zusätzliche Zeit wird darauf verwendet, eine weiterführende Ausbildung oder ein Studium abzuschließen. ${ }^{91}$ In diesem Fall läge das Berufseintrittsalter bei 22 Jahren. Das Renteneintrittsal-

\footnotetext{
${ }^{91}$ Der Einfachheit halber wird hier weiterhin angenommen, dass sich die Erträge von Ausbildung und Studium entsprechen. Eine längere Regelstudienzeit würde zwar das Eintrittsalter anheben, in der Regel aber auch zu gesteigerten Erträgen führen.
} 
ter und damit das Ende der Erwerbsperiode ist hier auf 65 Jahre festgesetzt. Welche dieser Alternativen finanziell attraktiver ist, hängt nun maßgeblich von den hiermit verbundenen Kosten und Erträgen ab.

Abbildung 10: Bildungsentscheidung und finanzieller Trade-off

Einkommen, Indirekte Kosten

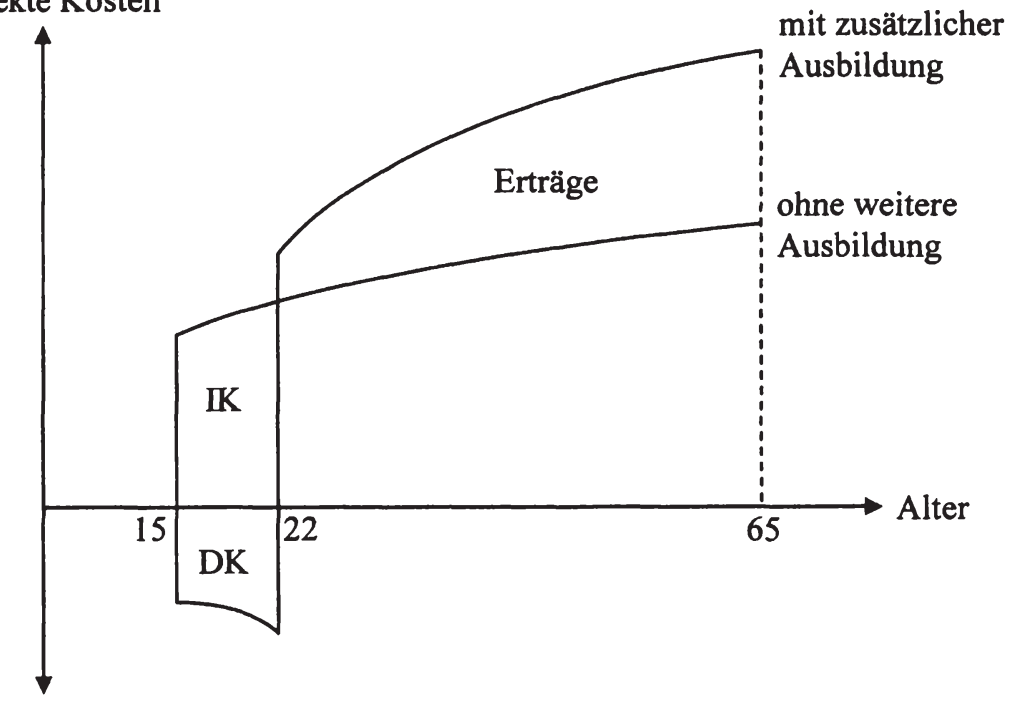

Direkte

Kosten

\section{IK: Indirekte Kosten}

\section{DK: Direkte Kosten}

Quelle: Vgl. Todaro und Smith (2003), S. 370.

In der Situation ohne weitere Ausbildung entstehen keine weiteren Kosten, es wird sofort ein Erwerbseinkommen erzielt. Mit zunehmendem Alter ergeben sich Lohnzuwächse, die aufgrund der fehlenden Ausbildung jedoch sehr gering ausfallen. Der Entscheidung, auf eine Ausbildung zu verzichten, steht die Alternative der Weiterbildung gegenüber. Analog zum ersten Fall ergibt sich bei Berufseintritt ein Erwerbseinkommen, das mit zunehmendem Alter ansteigt. Aufgrund der zusätzlichen Ausbildungsjahre setzen diese Erträge später ein, dafür 
sind hier stärkere Zuwachsraten realisierbar. ${ }^{92}$ Zudem fallen durch die Ausbildung im Gegensatz zur ersten Alternative noch direkte und indirekte Kosten an.

Die direkten Kosten decken sämtliche Kosten ab, die unmittelbar mit der Weiterbildung verbunden sind, etwa Schulgeld oder Studiengebühren. ${ }^{93}$ Die indirekten Kosten entsprechen den Opportunitätskosten in Höhe des entgangenen Erwerbseinkommens. Damit in Bildung investiert wird, muss der Gegenwartswert der zusätzlichen Erträge den Gegenwartswert der zusätzlichen Kosten übersteigen. Analog zu übrigen Investitionsentscheidungen lässt sich die Rentabilität von Bildungsinvestitionen daher anhand der Rendite bestimmen. ${ }^{94}$

Bils und Klenow (2000) zeigen in diesem Zusammenhang, dass das potentielle Lohnwachstum umso größer ist, je geringer das gegenwärtige Einkommensniveau ist. In Ländern mit geringem Durchschnittseinkommen und niedrigem Bildungsniveau führt zusätzliche Bildung folglich zu höheren Einkommenszuwächsen als in Ländern mit hohem Durchschnittseinkommen, da in Entwicklungsländern die Einkommensunterschiede zwischen einzelnen Personengruppen in der Regel größer ausfallen (vgl. Todaro und Smith (2003), S. 371f.). Allein unter Berücksichtigung der Ertragsseite würde diese Überlegung bedeuten, dass insbesondere in ärmeren Nationen ein positiver Anreiz zu mehr Bildung gegeben ist.

Unter Berücksichtigung der indirekten Kosten ist die Ertragsrate ärmerer Familien jedoch tatsächlich geringer als bei reichen Familien. Die Opportunitätskosten der Ausbildung sind zu hoch, wenn ärmere Familien für die Dauer der Ausbildung nicht auf das zusätzliche Einkommen verzichten können (vgl. Todaro und Smith (2003), S. 388). Wird also nicht der potentielle Einkommensanstieg, sondern die Rendite als Maßstab für die Bildungsinvestition angelegt, folgt hieraus, dass sich Bildung insbesondere dann lohnt, wenn bereits Vermögen vorhanden ist, da in diesem Fall der Einkommensverzicht finanziell leichter zu überbrücken ist.

92 Grundsätzlich gilt, dass Investitionen in die Bildung und damit in das Humankapital so lange zu positiven Erträgen führen, wie ein Produktivitätsanstieg zu verzeichnen ist (vgl. Schultz (1961), S. 8). Führt mehr Bildung zu einer gesteigerten Effizienz in den ökonomischen Produktionsprozessen und damit zu mehr Produktivität, ergeben sich hieraus im Zeitablauf entsprechende Lohnzuwächse.

${ }^{93}$ Die Darstellung folgt hier der Arbeit von Todaro und Smith (2003), in der die direkten Kosten mit zunehmendem Alter ansteigen. Für das deutsche Ausbildungswesen wäre ein konstanter Verlauf ebenso plausibel. Im Hinblick auf die Interpretation der Grafik ist diese Annahme jedoch nicht näher von Bedeutung.

${ }^{94}$ Siehe hierzu auch Psacharopoulos (1982). 


\subsection{Gesundheitsorientierte Determinanten von Bildungsinvestitionen}

Die Erläuterungen im vorangegangenen Abschnitt beziehen sich auf Bildungsinvestitionen mit einer sicheren Ertragsperiode. Die erwarteten Erträge werden somit ausschließlich durch die Effizienz der Bildungsinvestitionen determiniert, von Unsicherheiten über das zukünftige Arbeitsangebot wurde bislang abstrahiert. Tatsächlich können jedoch Erwartungen über Verdienstausfälle aufgrund von Krankheit die erwartete Rendite der Bildungsinvestitionen senken und somit den Anreiz erhöhen, auf weiterführende Bildung zu verzichten. Demgegenüber erhöht eine gute Gesundheit die Bildungsproduktivität, so dass der gewünschte Bildungskapitalstock mit geringerem Zeitaufwand erreicht werden kann. Die übrige Zeit kann in Form von Arbeit auf dem Markt angeboten werden, und eine höhere Arbeitszeit führt zu steigendem Einkommen. Bleibt der Zeitaufwand hingegen konstant oder wird sogar mehr Zeit in die Bildung investiert, steigt auch der Bildungskapitalstock, so dass ein höherer Lohn erzielt werden kann. ${ }^{95}$

Unter Sicherheit stehen den zukünftigen Bildungserträgen lediglich die anfänglichen, direkt mit der Bildung verbundenen Kosten sowie die Opportunitätskosten gegenüber, so dass hieraus die Rendite der Bildungsinvestitionen bestimmt werden kann. Unsicherheit führt hingegen dazu, dass die Entscheidung, in Bildungskapital zu investieren, verzerrt wird. Es ist zu erwarten, dass eine hohe Erkrankungswahrscheinlichkeit und damit verbunden zumindest zeitweilige Erwerbsunfähigkeit die zu Beginn des Erwerbslebens für Bildung aufgewendete Zeit senkt. Damit ist weiterhin zu erwarten, dass sich eine Verbesserung der individuellen Gesundheit zum Zeitpunkt der Ausbildungsentscheidung positiv auf die Akkumulation von Bildungskapital auswirkt. Eine hiermit verbundene Senkung von Fehlzeiten, die aufgrund von Krankheit anfallen, verlängert die Ertragsperiode aus den Bildungsinvestitionen und senkt so die Abschreibungsrate auf das Bildungskapital. Schlechte Gesundheit führt jedoch zu einer Verringerung des effektiven Arbeitsangebots während der Ertragsperiode und damit zu einer geringeren Rendite der Humankapitalinvestitionen.

Die direkten und indirekten Kosten, die zeitnah mit der Ausbildung verbunden sind, bleiben auch bei Unsicherheit über die zukünftige Gesundheit bestehen. Für den Fall, dass es zu Krankheit kommt, ergeben sich zusätzliche direkte Kosten in Höhe der Behandlungskosten. Zum Zweck einer vereinfachten Darstellung wird angenommen, dass sich diese in beiden Einkommenssituationen entsprechen, die Behandlung von Krankheiten also einkommensunabhängig erfolgt. Darüber hinaus ergeben sich zusätzliche indirekte Kosten in Höhe der ent-

${ }^{95}$ Dieser Zusammenhang gilt unter der Voraussetzung einer niedrigen Zeitdiskontierungsrate, die eine geringe Gegenwartspräferenz impliziert. 
gangenen Einkommenszuwächse, die ebenfalls bei der Bildungsentscheidung zu berücksichtigen sind.

Den Einfluss von Krankheit auf die optimale Bildungsinvestition beschreibt Abbildung 11. Sowohl die Einkommensfunktionen als auch die anfänglichen Kosten bleiben im Vergleich zu Abbildung 10 so lange unverändert, bis der Krankheitsfall eintritt. Ohne jeglichen Versicherungsschutz bedeutet dies, dass das Erwerbseinkommen jeweils auf Null absinkt und bei Genesung wieder auf das Ausgangsniveau ansteigt. Erst jetzt können wieder Einkommenssteigerungen aus der Bildung realisiert werden.

Abbildung 11: Finanzieller Trade-off unter Berücksichtigung von Krankheit

\section{Einkommen}

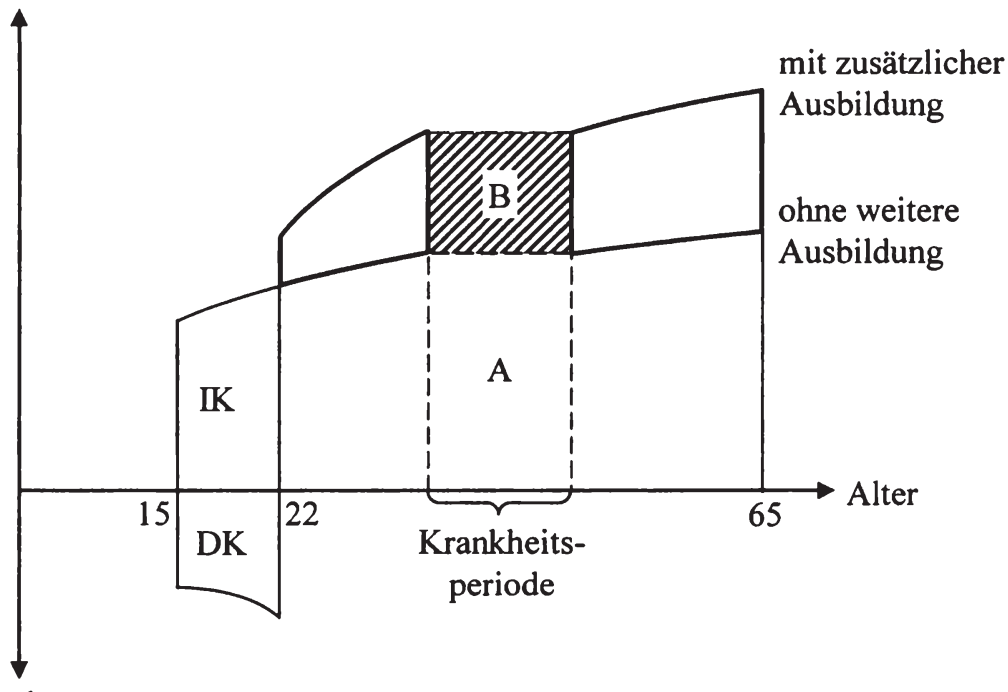

Direkte

Kosten

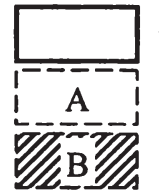

tatsächlich realisierte Einkommenszuwächse

krankheitsbedingte Einkommensverluste ohne Ausbildung zusätzliche Einkommensverluste mit Ausbildung

Quelle: Eigene Darstellung. 
Wurde auf eine Ausbildung verzichtet, kommt es zu Einkommensverlusten in Höhe von $\mathrm{A}$, während sich für Personen mit Ausbildung sogar direkte Einkommensverluste in Höhe von $\mathrm{A}+\mathrm{B}$ ergeben. Für beide Einkommenssituationen gilt darüber hinaus gleichermaßen, dass die tatsächliche Ertragsperiode umso kürzer ist, je länger der Krankheitszustand andauert. ${ }^{96}$

Für die Frage nach der Rentabilität von Bildungsinvestitionen ist zusätzlich zu berücksichtigen, welche Einkommenszuwächse erwartet worden sind und welche Einkommenszuwächse sich nun aufgrund des Krankheitsfalls tatsächlich ergeben. Dieser Zusammenhang ist in Abbildung 12 dargestellt. Das Einkommensniveau, welches sich bei dauerhafter Erwerbsfähigkeit eingestellt hätte, kann aufgrund der Krankheit nicht erreicht werden kann, da sich während der Krankheitsperiode keine Produktivitätszuwächse ergeben haben, die weitere Lohnsteigerungen rechtfertigen würden.

Die beiden weißen, dick umrandeten Flächen kennzeichnen erneut die tatsächlich realisierten Einkommenszuwächse, so dass sie mit den korrespondierenden Flächen in Abbildung 11 identisch sind. Die rechte der beiden Flächen wurde nach oben verschoben, bis der untere Rand mit der Einkommensfunktion ohne Ausbildung deckungsgleich ist. Die maximal möglichen Bildungserträge werden in Analogie zu Abbildung 10 über die Differenz der beiden Einkommensprofile bestimmt. Folglich bringt ein Krankheitsfall mit einhergehender Erwerbsunfähigkeit in der Endsumme einen Einkommensverzicht mit sich, welcher der diagonal schraffierten Fläche in Abbildung 12 entspricht.

Diese Abbildung zeigt allgemein, dass Krankheit das Verhältnis von Erträgen und Kosten der Bildung kleiner werden lässt. Ob und wie viel Zeit in Bildung investiert wird, hängt nicht mehr nur von den einzelnen Lohnprofilen ab, sondern auch von den erwarteten Verlusten im Erwerbseinkommen. Darüber hinaus bestimmt die Länge der Erwerbsperiode die Erträge der Bildungsinvestitionen und damit die Bereitschaft, zunächst auf Einkommen zu verzichten und Zeit für Bildungszwecke aufzuwenden. Ist mit Krankheit überdies nicht nur zeitweilige, sondern womöglich vollkommene Erwerbsunfähigkeit verbunden, sinkt der Bildungsanreiz weiter. Wird bereits frühzeitig das Eintreten eines Krankheitsfalls erwartet, können die bis dato realisierten Einkommenszuwächse die direkten und indirekten Kosten nicht mehr kompensieren, so dass es individuell rational ist, Bildungsinvestitionen $\mathrm{zu}$ unterlassen und frühzeitig erwerbstätig zu werden.

\footnotetext{
${ }^{96}$ Im Rahmen dieser Darstellung wird davon ausgegangen, dass sich aus der zeitweiligen Arbeitsunfähigkeit keine Produktivitätsverluste ergeben. Ein solches Szenario wäre jedoch ebenso denkbar, so dass das Einkommen nach der Krankheit zunächst unter dem vorherigen Einkommen läge.
} 
Ein ähnlicher Effekt kann einer geringen Lebenserwartung, über die die Lebensarbeitszeit bestimmt wird, zugeschrieben werden.

Abbildung 12: Entgangene Einkommenszuwächse durch Krankheit

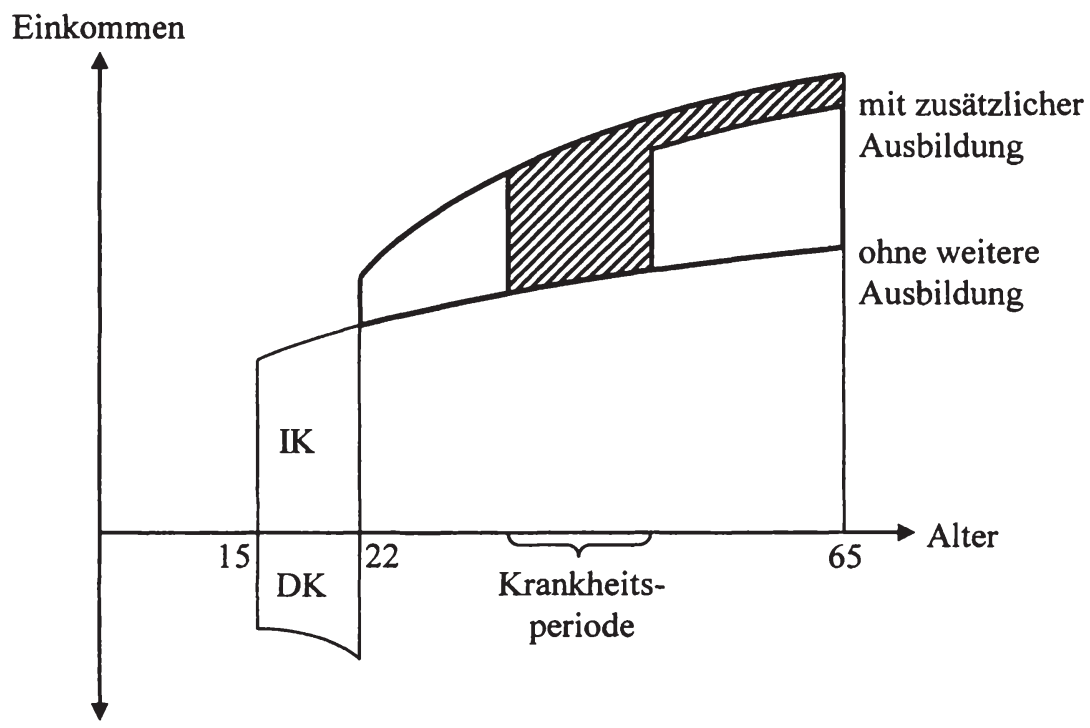

Direkte

Kosten

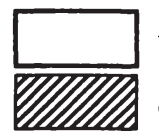

tatsächlich realisierte Einkommenszuwächse durch Krankheit entgangene Einkommenszuwächse

Quelle: Eigene Darstellung.

\subsubsection{Auswirkungen der Lebenserwartung auf die Bildungsentscheidung}

Die Überlegung, dass zwischen der Bildung auf der einen und der Lebenserwartung auf der anderen Seite eine komplementäre Beziehung besteht, geht auf Mushkin (vgl. 1962, S. 130-132) zurück. Mushkin untersucht in ihrer Arbeit den Einfluss einer hohen Sterblichkeitsrate von Kindern sowie einer geringen Lebenserwartung auf die Bildungsnachfrage. Hohe Kindersterblichkeit führt dazu, dass nur ein Teil der Kinder aus der Schule in die Erwerbsperiode überwechselt, so dass sich hieraus eine hohe gesellschaftliche Abschreibungsrate der Bildungsinvestitionen ergibt. Gleichzeitig liegen die durchschnittlichen Ausbildungskosten für jede erwachsene Arbeitskraft über den tatsächlichen Bildungs- 
ausgaben pro Kopf, so dass das Budget für Bildung nicht in adäquatem Maße zu Erträgen führt. Gleiches gilt für eine geringe Lebenserwartung, da diese die Ertragsperiode verkürzt und somit das Verhältnis der erwarteten Erträge zu den Kosten der Ausbildung negativ beeinflusst. Ein Absenken der Kindersterblichkeit würde folglich zu einem Anstieg in der Zahl qualifizierter Erwerbstätiger führen, die einer identischen Summe an Bildungsausgaben gegenübersteht. Ein genereller Anstieg der Lebenserwartung hingegen würde den relativen Zeitaufwand für Bildung verkürzen.

Eine ausführliche Untersuchung des Einflusses der Lebenserwartung auf das Arbeitsangebot findet sich zudem in Ram und Schultz (1979). Verbessert sich zunächst die Gesundheit, steigt die physische Belastbarkeit und die Zahl der Fehltage sinkt, so dass die effektive Arbeitszeit ansteigt. Bei gleich bleibendem Lohnsatz hätte dies Zuwächse im Einkommen zur Folge, ohne dass sich zunächst eine Veränderung hinsichtlich der Qualität der Arbeitskraft oder der Länge der Erwerbsperiode ergibt. Ein Anstieg in der Lebenserwartung führt nun dazu, dass sich die Investitionen in die Bildung über einen längeren Zeitraum amortisieren können. Dementsprechend besteht ein erhöhter Anreiz, in die Bildung zu investieren unter der Prämisse, dass Bildungsinvestitionen mit Investitionen in zukünftige Einkommen gleichzusetzen sind. Als Konsequenz hieraus ergibt sich eine Reallokation der Ressourcen, da mehr finanzielle Mittel für Bildungszwecke verwendet werden.

Problematisch ist ein Anstieg der Lebenserwartung dann, wenn sich hieraus Bevölkerungswachstum ergibt und der Arbeitsmarkt nicht gleichzeitig durch entsprechende Investitionen gestützt wird. In einem solchen Fall käme es zu einer sinkenden Kapitalintensität, da einer ansteigenden Erwerbsquote ein konstanter Kapitalstock gegenüberstehen würde. Dies hätte zum einen negative Auswirkungen auf die Höhe der Pro-Kopf-Ersparnisse, zum anderen steht gleichzeitig weniger Kapital für Investitionen zur Verfügung, so dass es immer schwerer wird, ein Minimum an Sozial- und Bildungseinrichtungen bereitzustellen. Konsequenzen hieraus wären ein geringeres Wirtschaftswachstum sowie ein Absinken des Pro-Kopf-Einkommens. ${ }^{97}$

\footnotetext{
${ }^{97}$ Bei Zhang et al. (2003) ist die anfängliche Lebenserwartung ein Indikator dafür, ob sich aus einem Anstieg der Lebenserwartung positive oder negative Wachstumseffekte ergeben. Für Länder mit hoher Sterblichkeit führt eine höhere Lebenserwartung zu mehr Wirtschaftswachstum, während sich dieser Effekt aufgrund des langfristig hiermit verbundenen DoubleAging-Problems mit zunehmender Lebenslänge ins Negative umkehrt (siehe hierzu auch Ulrich (2001)).
} 
Aus diesen Überlegungen folgt, dass die Infrastruktur in Form von Schulen und Universitäten gegeben sein muss, damit eine Befriedigung der Bildungsnachfrage in Quantität und Qualität möglich ist und damit die positiven Bildungsanreize einer erhöhten Lebenserwartung zu Wirtschaftswachstum führen. Problematisch ist zudem die geringe Akzeptanz von öffentlichen Bildungsausgaben als Investition in das Humankapital. Oftmals wird derartigen Bildungsausgaben eine konsumtive Wirkung zugeschrieben, ohne den investiven Charakter zu berücksichtigen. Gemäß dieser Argumentation würde es sich hierbei um eine Belastung der öffentlichen Haushalte handeln, der keine Erträge gegenüberstehen. Darüber hinaus würden nach dieser Auffassung die öffentlichen Mittel übrigen Investitionszwecken in Form von physischem Kapital entzogen werden. Tatsächlich führen diese Investitionen jedoch zu Erträgen, die der Wirtschaftlichkeit der gesamtwirtschaftlichen Produktionsprozesse zugute kommen und damit wiederum Kapital freisetzen.

Ram und Schultz (1979) kommen in ihrer Arbeit zu dem Ergebnis, dass ein Anstieg in der Lebenserwartung die Attraktivität von Bildungsinvestitionen erhöht. Folglich führt eine verbesserte Gesundheit nicht nur zu weniger Fehlzeiten und einer gesteigerten Produktivität per se, sondern auch zu einer verlängerten Ertragsperiode und damit zu mehr Bildung.

Zusammenfassend wirken sich Gesundheitsinvestitionen wie folgt auf den Arbeitsmarkt aus: Auf der einen Seite kann das individuelle Arbeitsangebot bei verbesserter Gesundheit ausgeweitet werden, so dass sich auch eine Ausweitung des gesamtwirtschaftlichen Arbeitsangebots ergibt. Auf der anderen Seite impliziert eine gesteigerte Arbeitsproduktivität eine größere betriebliche Nachfrage nach Arbeit im Verhältnis zum physischen Kapital, weshalb die aggregierte Arbeitsnachfrage ebenfalls ansteigt. In Kombination mit einem verlängerten Erwerbsleben aufgrund einer höheren Lebenserwartung führt Gesundheit schließlich dazu, dass Arbeit in Qualität und Quantität ansteigt.

Swanson und Kopecky (2000) zeigen ebenfalls, dass Individuen mit höherer Lebenserwartung einen größeren Anreiz haben, mehr Zeit in die Bildungskapitalakkumulation zu investieren. Dementsprechend ist bei steigender Lebenserwartung auch ein Ausbau der öffentlichen Bildungseinrichtungen zwingend notwendig. Umgekehrt führt die Bereitstellung von Bildungsmöglichkeiten nur dann zu einer positiven Resonanz, wenn die Lebens- und auch die Gesundheitserwartung entsprechend hoch sind. Andernfalls bietet zwar die Infrastruktur die Möglichkeit, Wachstum durch Bildung zu erreichen, diese würde jedoch aufgrund fehlender Anreize nicht hinreichend genutzt. Weiterhin betonen die Autoren die Vorteilhaftigkeit von Bildungsinvestitionen, indem über die gesteigerte Produktivität nicht nur für die Güterproduktion vermehrt Ressourcen zur Verfü- 
gung stehen, sondern auch für Investitionen in die Gesundheit. Hierzu zählen sowohl die sanitäre als auch die medizinische Versorgung, beispielsweise in Form von Impfungen oder besseren Behandlungsmöglichkeiten. Derartige öffentliche Investitionen können aufgrund ihrer langfristigen Wirkung auf die Gesundheit zu nachhaltigen Erträgen führen.

Auch wenn eine steigende Lebenserwartung die Bereitschaft zu mehr Bildung erhöht, existieren dennoch Restriktionen, die eine höhere Bildungsnachfrage verhindern (vgl. Kalemli-Ozcan et al. (2000)). Die Opportunitätskosten in Form von entgangenem Einkommen liefern einerseits Aufschluss über die Rendite der Bildungsinvestition. Andererseits müssen jedoch laufende Unterhaltskosten durch dieses Einkommen gedeckt werden, so dass nicht nur die Bereitschaft zu mehr Bildung eine Rolle spielt, sondern auch die finanzielle Umsetzbarkeit. Für den Fall, dass das eigene Vermögen oder das der Familie nicht ausreicht, um die Ausbildung zu finanzieren, müsste ein Kredit aufgenommen werden. Auf Basis eines hohen Sterblichkeitsrisikos ist die Wahrscheinlichkeit einer ordnungsgemäßen Rückzahlung jedoch sehr niedrig. Dies gilt insbesondere, wenn das Risiko in die Prämie mit eingerechnet wird. In diesem Fall wäre eine Kreditfinanzierung mit prohibitiv hohen Kosten verbunden, weshalb eine solche nahezu unmöglich würde.

Ebenfalls restriktiv wirkt sich die Gegenwartspräferenz auf die Bildungsinvestitionen aus. Je höher diese ist, desto stärker wird das aktuelle Einkommen gewichtet und desto höher sind die Opportunitätskosten der Bildung, die sich wiederum negativ auf die Rendite auswirken. Ein Anstieg in der Lebenserwartung hat nach Yaari (1969) und de la Croix und Licandro (1999) einen sinkenden Diskontierungsfaktor zur Folge, so dass zukünftiger Konsum stärker gewichtet wird. Ist die Lebenserwartung hingegen sehr gering, ist die Diskontierungsrate entsprechend hoch, woraus sich ein positiver Anreiz zu früher Erwerbstätigkeit ergibt. Gleichzeitig führt eine geringe Lebenserwartung zu einer hohen $\mathrm{Ab}$ schreibungsrate des Bildungskapitals, da sich individuelle Fertigkeiten nicht auf andere Arbeitskräfte übertragen lassen. Das Verhältnis von Kosten zu Erträgen der Bildungsinvestitionen wird demnach negativ beeinflusst (vgl. de la Croix und Licandro (1999), S. 256).

Swanson und Kopecky (vgl. 1999, S. 213) führen ihrerseits drei verschiedene Gründe an, warum steigende Lebenserwartung maßgeblich für die wirtschaftliche Entwicklung ist, sobald diese einen Anstieg in der gesund verbrachten Zeit und damit in der verfügbaren Arbeitskraft bedeutet: Erstens steigert eine höhere Lebenserwartung das Leistungsniveau der Arbeitskräfte und somit das Einkommen, indem nicht nur die Bereitschaft zu einer Schul- und Ausbildung ansteigt, sondern indem auch betriebsbedingte Weiterbildung bei einer hinreichend hohen 
Lebenserwartung an Attraktivität gewinnt. Als Konsequenz aus dem sowohl qualitativ als auch quantitativ angestiegenen Arbeitsangebot ergibt sich ein höheres Outputniveau und damit eine höhere Kapitalintensität. Der zweite Grund richtet sich auf die Bereitschaft, neue Technologien zu adaptieren. Die Annahme, dass sich eine Ökonomie nicht zwingend auf der Technologiegrenze befinden muss, begründen Swanson und Kopecky damit, dass sich Weiterbildung für die Anwendung neuer Technologien nicht lohnt, wenn die Lebensspanne zu kurz ist. Damit ist nicht nur die Bereitstellung von technischem Fortschritt für das Wachstum elementar, sondern es muss auch die Fähigkeit zur Anwendung bestehen. Aus dieser Überlegung folgt, dass die Übergangsgeschwindigkeit zwischen zwei Einkommensniveaus umso höher ist, je höher die Lebenserwartung ist, da neue Technologien viel schneller angenommen werden. Der dritte positive Effekt der Lebenserwartung bezieht sich auf die Bereitschaft zur Migration. Ist die Lebenserwartung unterschiedlich, kann es selbst bei perfekten Märkten mit freiem Güter- und Kapitalverkehr dazu kommen, dass sich die Outputniveaus einzelner Länder unterscheiden, da es bei mangelnder Migration zu einer Fehlallokation qualifizierter Arbeitskräfte kommt. Ist weiterhin die Produktivität in einzelnen Ländern und auch Regionen unterschiedlich, können sich die Löhne und damit die Einkommensniveaus nicht angleichen. ${ }^{98}$

Chakraborty (2003) zeigt einen positiven Zusammenhang zwischen der Lebenserwartung und dem Pro-Kopf-Einkommen. Die Richtung der Kausalität ist jedoch nicht eindeutig zu bestimmen. Auf der einen Seite kann eine geringe Lebenserwartung zu Armut führen, indem auf Grund der zu starken Diskontierung der Zukunft zu wenig in Bildung investiert wird und die Sparneigung zu gering ist. ${ }^{99}$ Auf der anderen Seite ermöglicht ein hohes Einkommen hohe Gesundheitsinvestitionen und damit einen Anstieg in der Lebenserwartung, während Armut die Fähigkeit, in die Gesundheit zu investieren, beschränkt. Hieraus folgt, dass sich ein geringes Einkommen und eine niedrige Lebenserwartung gegenseitig bedingen. Abbildung 13 zeigt diesen Zusammenhang für 109 Länder mit einem Pro-Kopf-Einkommen von 9.635\$ oder weniger im Jahr 1996.

Letztlich kann es sogar zu einer Armutsfalle kommen, wenn die Bildungsinvestitionen derart auf die geringe Lebenserwartung reagieren. Die Einkommensunterschiede würden langfristig bestehen bleiben, Länder mit unterschiedlichen Gesundheitsstandards könnten nicht im Einkommen konvergieren. Ein Angleichen der Lebensbedingungen ist bei ansonsten identischen Ökonomien nur dann möglich, wenn ausreichend Kapital zur Verfügung steht, um Investitionen in die

\footnotetext{
${ }^{98}$ In diesem Ansatz wird unterstellt, dass die Mobilität von Personen mit der Lebenserwartung ansteigt.

${ }^{99}$ Siehe hierzu auch Gersovitz (1983).
} 
Gesundheit zu ermöglichen und so die Lebenserwartung anzuheben, damit sich hieraus positive Bildungsanreize ergeben, die zu einem Produktivitätsanstieg und letztlich zu Einkommenswachstum führen.

Abbildung 13: Lebenserwartung und Pro-Kopf-Einkommen ${ }^{100}$

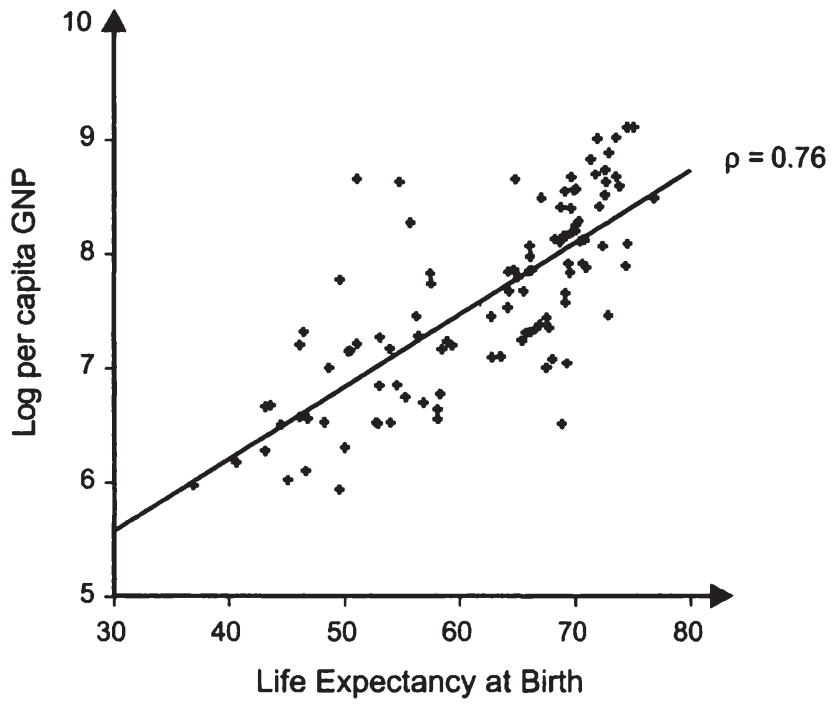

Source: World Bank (1998). Per capita GNP is measured in PPP\$.

These 109 low- and middle-income countries are those in which 1996 per capita GNP was $\$ 9,635$ or less.

Quelle: Chakraborty (2004), S. 120.

Kalemli-Ozcan ((2002) und (2003)) betont nicht nur die höhere Bereitschaft zu mehr Schulbildung, die mit einem Anstieg in der Lebenserwartung einhergeht, sondern auch die Bedeutung der Kindersterblichkeit für die Fertilität, woraus sich verschiedene demographische Effekte ergeben können. Besteht Unsicherheit über die Sterblichkeitsrate, ist die Zahl der überlebenden Kinder nicht vollständig planbar, es ergeben sich Verzerrungen hinsichtlich der tatsächlich überlebenden Kinderzahl. So kann es bei allgemein hoher Sterblichkeit dazu kommen, dass die Fertilität über der tatsächlich gewünschten Kinderzahl liegt. Hohe

${ }^{100}$ Der Korrelationskoeffizient $\rho$ lässt den Schluss zu, dass ein deutlich positiver Zusammenhang zwischen der Lebenserwartung bei Geburt und dem logarithmierten Pro Kopf-BIP besteht. Hieraus lässt sich jedoch keine Aussage über die Richtung der Kausalităt treffen. 
Geburtenzahlen sind daher als eine Versicherung der Eltern gegen eine hohe Kindersterblichkeit zu verstehen. Steigt nun die Überlebenswahrscheinlichkeit der Kinder an, kann sich die Fertilität nicht zeitnah anpassen, es kommt zu Bevölkerungswachstum. In diesem Fall führt ein Anstieg in der Lebenserwartung der Kinder nicht zu vermehrten Bildungsinvestitionen, da die ohnehin knappen Ressourcen nun auf mehr überlebende Kinder aufgeteilt werden müssen. ${ }^{101}$

Anders stellt sich die Situation bei einer bereits relativ hohen Überlebenswahrscheinlichkeit dar. Hier ist die Notwendigkeit der Eltern, sich gegen die hohe Mortalität zu versichern, relativ gering, so dass es zu einem Rückgang in den Geburtenzahlen kommen kann. Erst in diesem Fall setzt ein Absinken der Mortalität Ressourcen für die Erziehung und die Bildung der überlebenden Kinder frei, während gleichzeitig die Bereitschaft, in die Bildung der Kinder zu investieren, ansteigt. Der Einfluss einer geringeren Mortalität auf die demographische Entwicklung hängt somit maßgeblich von der bisherigen Überlebenswahrscheinlichkeit ab.

Änderungen in der Überlebenswahrscheinlichkeit lassen sich auf Änderungen in der medizinischen Versorgung sowie auf eine Veränderung des Einkommens zurückführen. Bei sehr niedrigem Einkommen führt ein Absinken der Sterblichkeit aufgrund des Vorsichtsmotivs zu einem Anstieg im Bevölkerungswachstum und damit c. p. zu geringerem Pro-Kopf-Einkommen. Aufgrund der fehlenden Ressourcen führt die steigende Lebenserwartung nicht zu mehr Schulbildung, so dass hier die Einkommenssituation trotz des Anstiegs in der Lebenserwartung nachhaltig auf niedrigem Niveau verbleibt. Letztlich steht durch das Bevölkerungswachstum sogar weniger Einkommen für die private Gesundheitsversorgung pro Kopf zur Verfügung, weshalb eine steigende Lebenserwartung c. p. mit einem Absinken des durchschnittlichen Gesundheitskapitalstocks verbunden sein kann. ${ }^{102}$

Bei hohem Einkommen senkt ein Anstieg der Lebenserwartung die Fertilität aufgrund der bereits erreichten höheren Überlebenswahrscheinlichkeit, so dass es zu mehr Bildungsinvestitionen und damit zu Wirtschaftswachstum kommen kann. ${ }^{103}$ Trotz eines niedrigen Einkommensniveaus wäre es armen Ländern da-

${ }^{101}$ Die Zahl der eigenen Kinder wäre nur dann mit Sicherheit bekannt, wenn nicht vorsorglich mehr Kinder geboren werden, sondern wenn es erst nach dem Tod eines Kindes zu einer neuen Schwangerschaft kommt (vgl. Kalemli-Ozcan (2003), S. 106).

102 Aufgrund dieser Zusammenhänge ist es in ärmeren Ländern zwingend notwendig, eine ausreichende staatliche Gesundheitsversorgung zu etablieren. Andernfalls kann das sinkende pro Kopf-Einkommen sogar dazu führen, dass die Lebenserwartung ebenfalls wieder absinkt.

${ }^{103}$ Dieses Argument impliziert, dass die Eltern die reduzierte Sterblichkeit zeitnah beobachten und bei ihrer Familienplanung berücksichtigen. 
her prinzipiell möglich, die Armutsfalle zu verlassen, sobald sich erneut eine Veränderung des Sterblichkeitsrisikos ergibt, da dann das Vorsichtsmotiv an Gewicht verliert und die für Bildung zur Verfügung stehenden Ressourcen ansteigen (vgl. Kalemli-Ozcan (2002), S. 422).

Aufgrund der geschilderten Zusammenhänge zwischen Sterblichkeit, Bildungsinvestitionen und Wachstum des Einkommens lassen sich Einkommenszuwächse jedoch nur durch Einkommenstransfers realisieren. Werden diese direkt an die Haushalte weitergegeben, muss sichergestellt sein, dass das zusätzliche Einkommen direkt der Gesundheit der Kinder zugute kommt. Eine gesundheitsorientierte Einkommensverwendung setzt jedoch erneut Bildung voraus. Werden Einkommenstransfers für den Aufbau eines allgemein zugänglichen Gesundheitssystems verwendet, kann die Gesundheit der Eltern wie auch die der Kinder verbessert werden, was letztlich in einem Anstieg der Lebenserwartung resultiert. Wichtig ist hierbei, dass sich die Verbesserung der Gesundheit ohne weitere finanzielle Belastung der Familien ergibt, um den Haushalten nicht die Lebensgrundlage zu entziehen. Die Einführung einer Krankenversicherung, die auf Umverteilung basiert, kann hier entsprechend helfen, die Geburtenraten und damit das Bevölkerungswachstum in Entwicklungsländern dauerhaft zu senken und somit die Voraussetzung für Wirtschaftswachstum zu schaffen.

\subsubsection{Die Bedeutung von Unsicherheit und Versicherung für die Bildungsinves- titionen}

Neben der Überlebenswahrscheinlichkeit ist der Grad der Sicherheit, mit der die Erträge von Bildungsinvestitionen realisiert werden können, für die Investitionsbereitschaft von Bedeutung. Besteht die Alternative zur unsicheren $\mathrm{Hu}$ mankapitalakkumulation in einer sicheren Geldanlage am Kapitalmarkt, bedeutet dies für risikoaverse Individuen, dass diese die sichere Kapitalmarktalternative vorziehen und so zu wenig in ihre Bildung investieren. Mögliche Ursachen für Unsicherheit sind beispielsweise durch unvollständige Information über die Qualität der Schulbildung sowie über die eigenen Fähigkeiten begründet, so dass sich die Wertigkeit des akkumulierten Bildungskapitals ex ante nicht bestimmen lässt. Zudem ist es wahrscheinlich, dass zukünftige Angebots- und Nachfragebedingungen auf dem Arbeitsmarkt nicht mit Sicherheit bekannt sind, so dass eine Fehlallokation der Ressourcen nicht ausgeschlossen werden kann (vgl. Kodde (1986)). Unsicherheit kann sich darüber hinaus auch aus dem Risiko, den Arbeitsplatz zu verlieren, ergeben (vgl. Rillaers (2001)). In diesem Fall verringert sich nicht nur die erwartete Ertragsperiode, sondern vormals akkumuliertes unternehmensspezifisches Wissen wird obsolet. Ein ähnlicher Effekt ergibt sich bei einem positiven Erkrankungsrisiko sowie bei Unsicherheit über die zu erwartende Lebenslänge, sollte hierdurch die Erwerbsperiode und nicht nur die Ruhestandsphase beeinflusst werden. 
Bildungskapital birgt stets ein höheres finanzielles Risiko in sich als physisches Kapital, da individuelle Fähigkeiten nicht diversifizierbar sind und daher nicht käuflich erworben oder veräußert werden können. Damit steht jedes Individuum bei seiner Bildungsentscheidung vor dem Trade-off einer höheren finanziellen Sicherheit auf der einen und einem hohen erwarteten Erwerbseinkommen auf der anderen Seite: Je allgemeiner die erworbene Bildung ist, desto geringer ist das Risiko, arbeitslos zu werden oder eine neue Stelle zu finden, und desto geringer sind auch die Opportunitätskosten von Krankheit in Form entgangenen Erwerbseinkommens. Andererseits lohnt sich Bildung gerade bei einem hohen Spezialisierungsgrad, wenn hiermit große Unterschiede in den erwarteten Einkommen verbunden sind (vgl. Levhari und Weiss (1974)). Je weiter das zu erwartende Maximaleinkommen demnach von dem Einkommen ohne Ausbildung entfernt ist, desto höher ist der Anreiz, in die Bildung zu investieren (vgl. Kodde $(1986)) .^{104}$

Zwar lassen sich unsichere Bildungserträge, die mit eigenen Anstrengungen verbunden oder von der Qualität der in Anspruch genommenen Ausbildung abhängig sind, nicht direkt versichern, es besteht in der Regel jedoch die Möglichkeit, eine Arbeitslosen- oder Krankenversicherung abzuschließen und so die Einkommensverluste im Eintrittsfall zu verringern. Da Bildung sehr zeitintensiv ist, kann in dieser Lebensphase kein oder nur wenig Erwerbseinkommen erzielt werden, so dass Investitionen unter Unsicherheit für risikoaverse Individuen zu niedrig ausfallen. Riskante, aber bei Erwerbstätigkeit sehr profitable Aufgaben werden daher eher angenommen, wenn eine finanzielle Absicherung verfügbar ist. Gleichzeitig verliert das Finanzkapital an Bedeutung für das Einkommen der Haushalte, so dass ein größerer Teil der Zeit in die Bildung investiert werden kann (vgl. Krebs (2003)).

Wird die Versicherung allerdings aufgrund von Moral Hazard nicht am Kapitalmarkt abgeschlossen, sondern durch den Wohlfahrtsstaat übernommen und mit Hilfe von Steuereinnahmen finanziert, wird zwar auf der einen Seite das Einkommensverlustrisiko gemindert, auf der anderen Seite sinkt aber auch der Anreiz, in Bildung zu investieren, da die Abhängigkeit vom eigenen Erwerbseinkommen abnimmt. Sobald das gewünschte zukünftige Einkommen oberhalb des durch die Versicherung garantierten Einkommens liegt, ist jedoch davon auszugehen, dass der erste Effekt den Zweiten überwiegt und Bildungsin-

104 Anderberg und Andersson (2000) unterscheiden Investitionen in Humankapital von ubrigen Investitionen ebenfalls dadurch, dass Unsicherheit hinsichtlich zukünftiger Erträge besteht, wăhrend es auf dem Kapitalmarkt stets eine risikofreie Option gibt. 
vestitionen so gefördert werden (vgl. Rillaers (2001) sowie Brown und Kaufold (1988))..$^{105}$

Eine empirische Überprüfung des Einflusses von Gesundheit auf die Entscheidung, Zeit in weiterführende Bildungsaktivitäten zu investieren, enthält die Arbeit von Perri (1984). Anhand von Befragungen über die Auswirkungen des Gesundheitszustandes auf die Ausbildungszeit wird gezeigt, dass Gesundheitsprobleme die Wahrscheinlichkeit einer längeren Ausbildung signifikant um 56\% senken. Im Durchschnitt ist die Ausbildungszeit derjenigen, die ihre eigene Gesundheit als schlecht einschätzen, um 2,4 Jahre kürzer als bei denjenigen, die ihre Gesundheit als exzellent einstufen (vgl. Perri (1984), S. 210f.). Mögliche Gründe hierfür sind mangelnde Effektivität sowie Unsicherheit über zukünftige Erträge der Bildungsinvestitionen. Eine Krankenversicherung kann demnach für Bildungsinvestitionen förderlich sein.

\subsection{Der Einfluss der Gesundheit auf die Produktivität der Bildungsinvestitio- nen}

In dem vorangegangenen Abschnitt besteht der positive Einfluss der Gesundheit auf die Bildung darin, die Überlebenswahrscheinlichkeit zu erhöhen bzw. die Dauer der Erwerbstätigkeit auszudehnen. Somit wurden bisher lediglich die quantitativen Aspekte einer verbesserten Gesundheit für die Akkumulation von Bildungskapital dargestellt. Darüber hinaus ist die Gesundheit auch für die Effektivität der Bildungsanstrengungen ein wesentlicher Faktor. Eine gute Ausbildung ist nur bei guter Gesundheit möglich, denn hohe Fehlzeiten aufgrund von Gesundheitsproblemen reduzieren die Effektivität sowie die Effizienz der Bildungsinvestitionen (vgl. Mushkin (1962), S. 130f.). Eine Verbesserung der Gesundheit würde die Produktionseffizienz der Zeitinputs und damit auch die erwarteten Erträge erhöhen. Demzufolge kann mit gleichem Zeitaufwand ein höherer Bildungskapitalstock erzielt werden (Alderman et al. (2001)). ${ }^{106}$

Eine Reihe empirischer Studien überprüft die Bedeutung der Gesundheit von Kindern für die Bildung anhand der Ernährung. Wolfe (1985) zeigt, dass Kinder, die unterernährt oder häufig krank sind, aufgrund einer geringeren Produktivität sowie aufgrund höherer Fehlzeiten die schulischen Leistungen gesunder Kinder nicht erreichen. In diesem Fall kann die vorhandene Bildungsinfrastruktur nicht

\footnotetext{
${ }^{105}$ Diese Quellen beziehen sich auf den Einfluss einer steuerfinanzierten Arbeitslosenversicherung auf die Bereitschaft zu Bildungsinvestitionen.

${ }^{106}$ Selbst wenn die Gesundheit keine direkten Produktivitătseffekte mit sich bringt, kann sie dennoch die Kosten der Bildung senken, so dass Ressourcen der Familie für die Ausbildung anderer Familienmitglieder freigesetzt werden (vgl. Alderman et al. (2001), S. 189).
} 
optimal genutzt werden. Darüber hinaus sind gesunde, gut ernährte Kinder im Erwachsenenalter produktiver, woraus sich zusätzlich Zeitverzögerungseffekte ableiten lassen (vgl. Webber (2002), S. 1634). Somit sollte es ein Ziel sein, möglichst gute Gesundheitsvoraussetzungen zu schaffen, um die Erträge der Bildungsbemühungen zu maximieren.

Currie und Hyson (1999) zeigen gleichermaßen, dass sich Untergewicht von Kindern besonders stark auf die Qualität ihrer Ausbildung auswirkt, darüber hinaus jedoch auch die zukünftige Gesundheit sowie die Teilnahme am Arbeitsmarkt negativ beeinflusst werden. In der Studie von Currie und Hyson werden bei der Geburt untergewichtige Kinder von Familien mit geringem sozioökonomischem Status mit untergewichtigen Kindern besser situierter Familien verglichen, um Verzerrungen durch unterschiedliches Investitionsverhalten auszuschließen. Im Ergebnis ergibt sich jedoch für beide Stichproben das gleiche Resultat: Es zeigt sich, dass sich Unterschiede in den Bildungsniveaus einzelner sozialer Gruppen sogar angleichen, wenn die Gesundheit bereits bei der Geburt durch ein zu geringes Körpergewicht beeinträchtigt wird. ${ }^{107}$ Hieraus folgt, dass Gesundheitsprobleme sowohl die für Bildung verfügbare Zeit als auch die Bildungsproduktivität und damit den erreichten Bildungsgrad nach einer vorgegebenen Periode mindern können, wodurch Einkommenseinbußen in zukünftigen Perioden in Kauf genommen werden müssen (vgl. Edwards und Grossman (1979), S. 275). ${ }^{108}$

Case et al. (2003) stellen die Hypothese auf, dass nicht unbedingt der sozioökonomische Status im mittleren Alter für die Gesundheit entscheidend ist, sondern dass das Einkommen der Familie in der Kindheit eine weitaus größere Rolle spielt. So verfügen Kinder ärmerer Familien über einen schlechteren Gesundheitszustand, geringere Investitionen in das Bildungskapital und eine schlechtere zukünftige Gesundheit, was alles in allem zu einem geringeren zukünftigen Einkommen führt. Diese Effekte werden schließlich an die eigenen Kinder weitergegeben, weshalb ein Verlassen einer schlechten Einkommensposition aus eigener Kraft kaum möglich ist.

\footnotetext{
107 Als Indikatoren für die Gesundheit werden chronische Gesundheitsprobleme, die Körpergröße sowie das Gewicht bei der Geburt verwendet. Während chronische Krankheiten sowohl angeborene als auch erst entstandene Gesundheitsdefizite widerspiegeln, reflektiert die Körpergröße die Ernăhrung, wobei die Körpergrößen der Eltern jeweils als Kontrollvariablen aufgenommen werden. Die Effekte für die Akkumulation von Bildungskapital sind jeweils statistisch signifikant negativ.

${ }^{108}$ Edwards und Grossman (1979) weisen zudem darauf hin, dass dieser Zusammenhang selbst dann gilt, wenn der Intelligenzquotient ebenso wie das häusliche Umfeld zum Zweck der besseren Vergleichbarkeit konstant gehalten wird.
} 
Die empirische Überprüfung von Case et al. (2005) liefert folgende Resultate: Zunächst führt schlechte Gesundheit dazu, dass die Wahrscheinlichkeit sinkt, ein bestimmtes Bildungsniveau $\mathrm{zu}$ erreichen. Zudem ist der Einfluss einer Krankheit im Kindesalter auf das Erwerbseinkommen signifikant negativ. So führt eine Gesundheitsbeeinträchtigung im Alter von 7 Jahren dazu, dass das Einkommen eines 42-Jährigen im Schnitt um 5,6 Prozent niedriger ausfällt als bei guter Gesundheit. Der Einfluss einer chronischen Krankheit im Alter von 16 Jahren bedingt eine Einkommenssenkung um 4 Prozent. Dieser Effekt lässt sich zum einen durch geringere Arbeitszeiten bzw. höhere Fehlzeiten erklären, die ihrerseits eine geringere Berufserfahrung und damit ein geringeres Einkommen zur Konsequenz haben. Zum anderen ist das Lohnwachstum aufgrund einer niedrigeren Arbeitsmarktproduktivität geringer, die sich einerseits aus einer schlechteren Gesundheit im Erwachsenenalter und andererseits aus einem geringeren Wissenskapitalbestand ableitet. ${ }^{109}$

Eine Verbesserung der Ernährung von Kindern führt demnach zu einer Verbesserung der Lernfähigkeit und damit zu höheren Humankapitalerträgen (vgl. Alderman et al. (2001), S. 185). Gesunde Ernährung begünstigt darüber hinaus ein Absinken der Mortalität und damit einhergehend einen Anstieg in der Lebensarbeitszeit sowie des Lebenseinkommens (vgl. Schultz (1997). Hieraus folgt, dass die Auswirkungen einer guten Gesundheit sowohl auf individueller als auch auf aggregierter Ebene in der Jugend langfristiger Natur sind, indem hierüber der verfügbare Bildungskapitalbestand determiniert wird.

${ }^{109}$ Entgegen der bereits genannten Arbeiten können Shakotko et al. (1981) keinen signifikanten Einfluss exzessiver Fehlzeiten auf die schulischen Leistungen belegen. Vielmehr gehen die Autoren davon aus, dass vor allem der Bildungsstand der Mutter die Bildung ihrer Kinder determiniert, wobei hier zum einen genetische Faktoren, zum anderen aber auch das Verhalten der Mutter eine bedeutende Rolle spielen. 


\section{Ein theoretisches Modell zum Einfluss der Gesundheit auf die Bildung}

\subsection{Die optimale Bildungsentscheidung unter Sicherheit}

In dem folgenden Zwei-Perioden-Modell steht jedes Individuum vor dem Entscheidungsproblem, einen Teil der Arbeitszeit in der ersten Periode in Bildung zu investieren, um in der zweiten Periode aufgrund von produktivitätsbezogenen Lohnsteigerungen über ein höheres Einkommen verfügen zu können. Analog zu Brown und Kaufold (1988) besteht keine Unsicherheit über den Bildungserfolg, d. h. die Produktionsfunktion für Bildungskapital ist exogen und bekannt. Neben der individuellen Fähigkeit, Bildungskapital zu akkumulieren, sind die Qualität der Inputgüter sowie die Restriktionen und Möglichkeiten, die der institutionelle Rahmen bestimmt, Elemente der Bildungsproduktionsfunktion (vgl. Ben-Porath (1967), S. 352). Weiterhin gilt, dem Modell von Ben-Porath folgend, dass $\mathrm{Hu}$ mankapital als solches nicht beschafft werden kann, sondern dass Individuen in ihrer Jugend unter Verzicht auf Einkommen in die Bildung investieren müssen, während die Erträge erst später realisiert werden können. Eine Kreditaufnahme zu Bildungszwecken ist ebenfalls nicht möglich, da die Kapitalmärkte unvollkommen sind. ${ }^{110}$ Es wird zusätzlich vereinfachend angenommen, dass kein Vermögen in der ersten Periode existiert.

Die dem Individuum insgesamt zur Verfügung stehende Zeit wird ausschließlich zwischen Bildung und Arbeit alloziert mit dem Ziel, den abgezinsten Wert aller zukünftigen Einkommen zu maximieren. Freizeit wird in diesem Modell nicht berücksichtigt. Weiterhin gilt zunächst, dass keinerlei Unsicherheit über das Arbeitsangebot in der zweiten Periode besteht, $d . h$. in beiden Perioden verfügt das Individuum über ausreichend gute Gesundheit. Es wird angenommen, dass die insgesamt verfügbare Zeit in der zweiten Periode ausschließlich für Arbeit aufgewendet wird. Es wird keine weitere Zeit in Bildung investiert, da Erträge nicht mehr realisiert werden können. Zudem wird das Einkommen in der zweiten Periode vollständig dem Konsum zugeführt, d. h. es besteht kein Vererbungsmotiv.

In der ersten Periode entscheidet jedes Individuum über die Höhe der in Bildung investierten Zeit $b$ sowie über die Höhe der Ersparnisse $S$, der Lohnsatz $w$ ist exogen gegeben und bekannt. Analog zu der Arbeit von Levhari und Weiss (1974) wird angenommen, dass Bildungsinvestitionen ausschließlich zeitintensiv sind und dementsprechend keine weiteren Ressourcen binden, so dass die

${ }^{110}$ Diese Annahme ist durch die Unsicherheit über die zukünftigen Erträge begründet. Es ist erstens möglich, dass die Ausbildung nicht erfolgreich abgeschlossen wird, und zweitens bestehen ex ante keine Informationen über das tatsächliche zukünftige Gehalt. 
Opportunitätskosten der Bildung dem entgangenen Lohn entsprechen. Für den Konsum in der ersten Periode $c_{1}$ gilt

$$
c_{1}=(1-b) w-S \quad b \in[0,1) \text {. }
$$

Da die verfügbare Zeit ausschließlich für Arbeit oder Bildung aufgewendet werden kann, wird die gewählte Arbeitszeit über den Term (1-b) bestimmt. Unter Berücksichtigung der Vermögensrestriktion ist $b<1, \mathrm{~d}$. h. es wird nicht die insgesamt verfügbare Zeit in Bildung investiert. Ein Teil der Zeit wird in jedem Fall für Arbeit aufgewendet, da sich hieraus nicht nur das Erwerbseinkommen ableitet, sondern auch der Konsum der ersten Periode sowie die Höhe der Ersparnisse, die den Konsum in der zweiten Periode mitbestimmen. ${ }^{111}$ Hingegen ist es möglich, keinerlei Bildungsinvestitionen durchzuführen, so dass die gesamte Zeit für Arbeit aufgewendet werden $\operatorname{kann}(b=0)$.

Das Einkommen in der zweiten Periode setzt sich zum einen aus den verzinsten Ersparnissen und zum anderen aus dem Erwerbseinkommen zusammen:

$$
c_{2}=(1+r) S+[1+\varphi] w .
$$

Die Verzinsung erfolgt mit dem Marktzinssatz $r$, der exogen gegeben und konstant ist, $d$. h. es besteht keine Unsicherheit über die Zinseinkünfte in der zweiten Periode. Das Erwerbseinkommen wird durch den Lohnsatz sowie die Produktivitätskomponente ${ }^{112}$

$$
\varphi \equiv \alpha(h) \beta(b)
$$

bestimmt. Diese Funktion zeigt, wie sich die in die Bildung investierte Zeit $b$ sowie der individuelle Gesundheitszustand $h$ auf die Produktivität in der Folgeperiode auswirken. Der Lerneffizienzparameter $\alpha(h), \alpha^{\prime}>0$, gibt zum einen Aufschluss darüber, wie sich die Lernfähigkeit mit der Gesundheit verändert, zum anderen wird hierüber der direkte Einfluss der Gesundheit auf die individuelle Arbeitsproduktivität abgebildet. Der zweite Parameter in Definition (7.3) gibt an, mit welcher Intensität Zeitaufwand in nutzbares Bildungskapital umge-

\footnotetext{
${ }^{111}$ Die Integration von Vermögen in das Modell würde seine Aussagekraft nicht weiter beeinflussen. Die Bedeutung von Unsicherheit furr die Akkumulation von Bildungskapital würde lediglich abgeschwächt (siehe hierzu Levhari und Weiss (1974)).

${ }^{112}$ In Anlehnung an Glewwe (2002), S. 438.
} 
wandelt werden kann. Dieser Parameter kann daher auch als Zeitintensitätsparameter bezeichnet werden.

Ergeben sich zwischen beiden Perioden keinerlei Veränderungen in der Gesundheit und wird zudem keine Zeit in die Bildung investiert, so folgt hieraus für die Produktivität, dass diese sich im Zeitablauf nicht verändert, d. h. $\varphi=0$. In diesem Fall wäre das Erwerbseinkommen in beiden Perioden absolut identisch. Sobald jedoch Zeit in die Bildung investiert wird, ergeben sich hieraus Produktivitätseffekte, die in der zweiten Periode zu einem Anstieg im Lohnsatz führen. Wie stark diese Produktivitätseffekte ausfallen, hängt maßgeblich vom Zeitintensitätsparameter $\beta(b)$ ab, so dass hierüber Unterschiede in den individuellen Fähigkeiten abgebildet werden können. ${ }^{113}$

Für jeden beliebigen Zeitanteil $0<b<1$ wird angenommen, dass dieser einen Anstieg im Bildungskapital und damit in der Produktivität mit sich bringt, d. h. $\partial \beta / \partial b>0$. Analog zu Ben-Porath (1967), Levhari und Weiss (1974) und Schäper (2003) unterliegt die Produktion von Bildungskapital abnehmenden Grenzerträgen $\left(\partial^{2} \beta / \partial b^{2}<0\right)$. Jede zusätzlich investierte Zeiteinheit bringt folglich immer geringere Lohnzuwächse mit sich.

Die Gesundheit ist in dem vorliegenden Modell aus Vereinfachungsgründen exogen gegeben, d. h. der Gesundheitszustand am Ende der ersten Periode lässt sich nicht durch Investitionen während der ersten Periode beeinflussen. Veränderungen der Gesundheit sind daher nicht das Ergebnis zielgerichteter Entscheidungen, sondern sie ergeben sich vielmehr als externe Effekte von Konsumentscheidungen.

Die Individuen treffen ihre Bildungsentscheidung, indem sie ihren Nutzen maximieren. Die Nutzenfunktion ist additiv separabel in der Zeit und enthält als einziges Argument den Konsum der jeweiligen Periode, das Bildungskapital und die Gesundheit sind nicht direkte Elemente der Nutzenfunktion: ${ }^{114}$

${ }^{113}$ Neben der investierten Zeit sind noch weitere Faktoren in der Bildungsproduktionsfunktion enthalten. Zusätzlich zu genetischen Faktoren spielen auch die Qualität der Bildungseinrichtungen sowie das häusliche Umfeld und der sozioökonomische Status der Eltern eine Rolle (vgl. Edwards und Grossman (1979), S. 274). Diese Faktoren sind jedoch durch die einzelnen Individuen nicht beeinflussbar und entziehen sich daher jeden Entscheidungskalküls. Damit bleibt weiterhin die in die Bildung investierte Zeit die einzige Bildungsvariable, die direkt in einen Entscheidungsprozess eingebunden wird.

114 Von einem Diskontierungsfaktor, der die Zeitpräferenz widerspiegelt, wird in diesem Modell abstrahiert. Somit wird angenommen, dass beide Perioden identisch gewichtet werden. Eine stärkere Gewichtung der Gegenwart aufgrund von Unsicherheit würde den Konsum in 


$$
\max _{S, b} u\left(c_{1}\right)+u\left(c_{2}\right)
$$

wobei die Nutzenfunktionen jeweils strikt konkav sind mit $u_{t}^{\prime}>0$ und $u_{t}^{\prime \prime}<0$. Die Bedingungen erster Ordnung lauten

$$
\begin{aligned}
& S: \quad u_{1}^{\prime}=(1+r) u_{2}^{\prime}, \\
& b: \quad u_{1}^{\prime}=\alpha \beta_{2}^{\prime} u_{2}^{\prime} .
\end{aligned}
$$

Für diese beiden Bedingungen gilt, dass der Nutzenentgang aufgrund des geringeren Einkommens in der ersten Periode dem jeweiligen Nutzenzuwachs in der zweiten Periode entsprechen muss. Es wird jeweils Konsumglättung betrieben. Aus den Bedingungen (7.5) und (7.6) folgt

$$
\beta^{\prime}=\frac{1+r}{\alpha} \text {. }
$$

Gleichung (7.7) zeigt, dass die optimale Grenzproduktivität der Bildung und damit der optimale Zeitaufwand für Bildung in der ersten Periode zum einen davon abhängt, wie hoch der Marktzinssatz ist und zum anderen über den Lerneffizienzparameter $\alpha$ bestimmt wird, der angibt, mit welcher Effizienz das Gelernte in Produktivität umgesetzt werden kann. Ein hoher Marktzins impliziert c.p. auch eine hohe Grenzproduktivität der Bildung $\beta$, so dass nur wenig Zeit in Bildung investiert wird. Eine hohe Effizienz $\alpha$ führt hingegen dazu, dass Produktivitäts- und hiermit verbundene Lohnsteigerungen mit geringerem Bildungszeitaufwand erreicht werden können, während die Rendite von Kapitalmarktinvestitionen in Form des Zinssatzes $r$ unverändert bleibt. Folglich ist Bildung bei hoher Lerneffizienz auch mit höheren finanziellen Anreizen verbunden, während Arbeit in der ersten Periode c. p. an finanzieller Attraktivität einbüßt.

Abbildung 14 zeigt den Zusammenhang zwischen dem Zeitaufwand für Bildungszwecke $b$ und den Grenzerträgen von Bildung und Ersparnissen $(\beta, 1+r)$ für einen beliebigen Lerneffizienzparameter $\alpha$. Auf der Abszisse ist der Zeitaufwand fur die Bildungsinvestitionen abgetragen, auf der Ordinate die Grenzerträge der Kapitalarten Real- und Bildungskapital. Der Grenzertrag der Erspar-

der ersten Periode aufwerten und sich folglich sowohl auf die Bildung als auch auf das Sparverhalten negativ auswirken. 
nisse wird ausschließlich durch die Verzinsung bestimmt. Die Zinsen sind unabhängig von der in die Bildung investierten Zeit und damit konstant. Die Grenzproduktivität der Bildung ist hingegen bei konstantem $\alpha$ mit zunehmendem Zeitaufwand abnehmend. Der Schnittpunkt dieser beiden Kurven kennzeichnet das Optimum, da sich hier die Grenzerträge beider Kapitalarten entsprechen. Steigt der Marktzins von $r_{0}$ auf $r_{1}$, verschiebt sich die Zinsgerade nach oben, so dass ein neues Gleichgewicht $b_{o p t}^{\prime}$ erreicht wird. Aufgrund der Zinserhöhung werden Investitionen am Kapitalmarkt lohnender, so dass weniger Zeit in die Bildung investiert wird.

Abbildung 14: Die optimale Bildungsinvestition unter Sicherheit

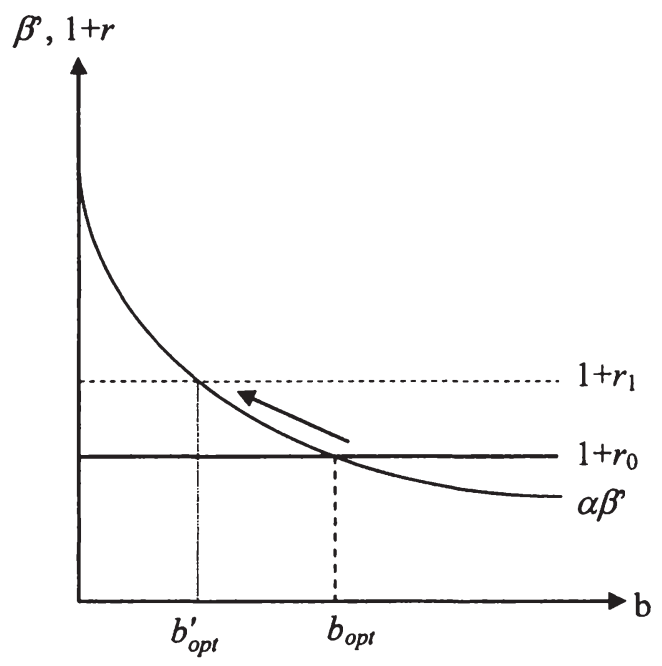

Quelle: Eigene Darstellung.

\subsection{Investitionen unter Unsicherheit ohne Krankenversicherungsschutz}

Nun ist die Annahme vollkommener Sicherheit sehr vereinfachend, denn die Erwerbsfähigkeit der einzelnen Individuen wird maßgeblich durch ihre Gesundheit determiniert. Folglich kann keine absolute Sicherheit über das Arbeitsangebot in der zweiten Periode bestehen, solange ein positives Erkrankungsrisiko existiert. Diese Unsicherheit hat für die Bildungsinvestitionen die Konsequenz, dass auch die Erträge der Bildung unsicher werden. Positive Erträge lassen sich jedoch nur bei ausreichender Gesundheit realisieren. Führt eine Erkrankung hingegen zu Erwerbsunfähigkeit, stehen den Investitionskosten in Höhe des entgangenen Erwerbseinkommens der ersten Periode keinerlei Erträge gegenüber. 
In diesem Abschnitt wird zunächst angenommen, dass keine Krankenversicherung existiert. Aus diesem Grund sind sämtliche Gesundheitsausgaben zur Behandlung der Erkrankung in der zweiten Periode privat über die Ersparnisse zu finanzieren. ${ }^{115}$

Die dem Individuum in der ersten Periode zur Verfügung stehende Zeit wird erneut ausschließlich zwischen Bildung und Arbeit aufgeteilt. ${ }^{116}$ Für die zweite Periode wird aufgrund einer fehlenden weiteren Ertragsperiode ebenfalls angenommen, dass die insgesamt verfügbare Zeit bei ausreichender Gesundheit ausschließlich für Arbeit aufgewendet wird. Bei Krankheit folgt jedoch Erwerbsunfähigkeit. Analog zu Kapitel 5 existieren nach wie vor nur zwei mögliche Gesundheitszustände (vgl. Ehrlich und Becker (1972), S. 625): Liegt die individuelle Gesundheit $h$ über dem kritischen Schwellenwert $h_{k}$, dann ist das Individuum gesund und damit in der zweiten Periode erwerbsfähig, es kann Erwerbseinkommen erzielt werden. Bei einem Gesundheitszustand unterhalb des kritischen Wertes reduziert sich das Arbeitsangebot hingegen auf Null, so dass der Konsum in dieser Periode ausschließlich über die Ersparnisse gedeckt wird. ${ }^{117}$ Der Konsum der zweiten Periode ist damit direkt von der individuellen Gesundheit abhängig.

Ziel der Individuen ist es, den Erwartungsnutzen zu maximieren. Dieser bestimmt sich aus dem Konsum in der ersten Periode sowie aus dem erwarteten Konsum der zweiten Periode. Bezeichnet nun $h$ den individuellen Gesundheitszustand am Ende der ersten Periode, dann gibt $\pi(h) \in(0,1)$ die Wahrscheinlichkeit einer zweiten Erwerbsperiode an mit $\partial \pi / \partial h>0$ und $\partial^{2} \pi / \partial h^{2}<0 .{ }^{118} \mathrm{Je}$

115 Angelehnt an das Modell von Kalemli-Ozcan et al. (2000) existiert kein Rentensystem, weshalb in der zweiten Periode keine weitere finanzielle Absicherung besteht. Somit wird angenommen, dass in der ersten Periode in jedem Fall ein Teil des Einkommens gespart wird.

${ }^{116}$ Ist ein Individuum bereits in der ersten Periode krank, so besteht keine Möglichkeit, Zeit in Bildung und Arbeit zu investieren. Die gesamt verfügbare Zeit wird für Investitionen in die Gesundheit aufgewendet. Damit wird dieser Fall in der nachfolgenden Analyse nicht weiter berücksichtigt.

117 Schlechte Gesundheit umfasst in diesem Zusammenhang sämtliche Gesundheitszustände, bei denen Arbeit nicht mehr möglich ist, also beispielsweise Krankheit oder Verletzung. Gesundheitsbeeintrăchtigungen, die nicht mit Erwerbsunfähigkeit einhergehen, werden uber den Produktivitătsparameter $\varphi$ abgebildet.

118 Williams (1979) betrachtet nicht die Gesundheit als Determinante fur die Unsicherheit, sondern die Bildungsproduktivităt, die Abschreibungsrate der individuellen Făhigkeiten, die zukünftig realisierbaren Löhne sowie die Zinserträge. Auch wenn derartige Unsicherheiten ebenfalls eine wesentliche Rolle fulr die Bildungsentscheidung spielen, soll an dieser Stelle davon abstrahiert werden, um die Bedeutung der Gesundheit für die Akkumulation von Bildungskapital isoliert darstellen zu können. Anderberg und Andersson (2000) weisen zudem darauf hin, dass Humankapitalinvestitionen nicht nur ein Einkommensrisiko verursachen, 
besser der Gesundheitszustand am Ende der ersten Periode ist, desto wahrscheinlicher wird es, dass in der zweiten Periode Arbeit angeboten werden kann. Darüber hinaus wird angenommen, dass stets ein Restrisiko einer Erkrankung in der zweiten Periode verbleibt, d. h. $\pi(h)<1$. Dieses Restrisiko lässt sich über Verletzungen, die zu Arbeitsunfähigkeit führen, erklären, da Verletzungen unabhängig vom aktuellen Gesundheitszustand auftreten können. Auch können am Ende der ersten Periode negative Externalitäten, beispielsweise das Auftreten eines neuen Virus, dazu führen, dass die zweite Periode trotz eines zuvor guten Gesundheitszustands krank verbracht wird. In dem nachfolgenden Modell ist die Gesundheit exogen gegeben, d. h. der Gesundheitszustand am Ende der ersten Periode lässt sich nicht durch Investitionen während der ersten Periode beeinflussen. ${ }^{119}$

Unter Verwendung einer von Neumann-Morgenstern Nutzenfunktion ${ }^{120}$ ergibt sich folgendes Optimierungsproblem:

$$
\max _{S, b} u\left(c_{1}\right)+\pi(h) u\left(c_{2 G}\right)+[1-\pi(h)] u\left(c_{2 K}\right)
$$

unter den Nebenbedingungen

$$
\begin{aligned}
& c_{1}=(1-b) w-S \quad b \in[0,1), \\
& c_{2 G}=(1+r) S+[1+\varphi] w,
\end{aligned}
$$

sondern dass diese auch einen Versicherungseffekt mit sich bringen, da beispielsweise die Wahrscheinlichkeit einer Erkrankung mit zunehmender Bildung geringer wird. Folglich wäre die Erkrankungswahrscheinlichkeit nicht nur von der Gesundheit, sondern auch von der Bildung abhängig. Diese Überlegung bietet eine Erweiterungsmöglichkeit des Modells, die hier aufgrund der zunehmenden Komplexität nicht weiter verfolgt werden soll.

119 Andernfalls wären die Investitionszeit sowie der Konsum von Gesundheitsleistungen im Rahmen der Gesundheitsproduktionsfunktion ebenfalls als Entscheidungsvariablen zu berücksichtigen.

${ }^{120}$ Es wird unterstellt, dass der Nutzen ausschließlich vom Konsumniveau in den jeweiligen Perioden abhängt. Ebenso wäre es gemäß der Arbeit von Grossman (1972) denkbar, der Gesundheit eine Nutzen stiftende Funktion zuzuschreiben. Zusätzlich wird erneut von Diskontierungsfaktoren abstrahiert. Blinder und Weiss (1976, S. 451) weisen darauf hin, dass unter Berücksichtigung von Zeitpräferenzen in einer Periode weniger Zeit in Bildung investiert wird, falls hierfür auf Freizeit verzichtet werden muss. Ebenso ist anzunehmen, dass Zeitdiskontierung zu einer stärkeren Gewichtung der Arbeitszeit in der ersten Periode fuhrt mit dem Effekt, dass die Bildungsinvestitionen zurückgehen. 


$$
c_{2 K}=(1+r) S-L .
$$

Den Individuen wird Risikoaversion unterstellt. Die Nutzenfunktion ist erneut streng konkav mit $u_{t}^{\prime}>0$ und $u_{t}^{\prime \prime}<0$. Die Nebenbedingung (7.9) beschreibt den Konsum der ersten Periode, der zu Gleichung (7.1) unverändert bleibt. Die verfügbare Zeit wird weiterhin für Arbeit und Bildung aufgewendet, während ein Teil des Erwerbseinkommens gespart wird. Auch für den Konsum im gesunden Zustand gilt, dass sich dieser nicht von der Sicherheitssituation unterscheidet (vgl. Gleichung (7.2)).

Bei Krankheit in der zweiten Periode wird der Konsum aus den verzinsten Ersparnissen abzüglich des monetär bewerteten Krankheitsschadens $L$ bestimmt (Gleichung (7.11)). Da Verschuldung in diesem Modell nicht zulässig ist, können in der zweiten Periode keine Kredite aufgenommen werden, um die Ausgaben für medizinische Leistungen bei Krankheit zu finanzieren. Damit sämtliche Ausgaben bei Krankheit gedeckt sind, muss sich die Summe der verzinsten Ersparnisse folglich mindestens auf die Schadenshöhe belaufen, weshalb $(1+r) \mathrm{S} \geq L$ gelten muss. Da ausschließlich das Erwerbseinkommen durch die Möglichkeit einer Erkrankung beeinflusst wird, sind die Ersparnisse sowie deren Zinserträge in beiden Perioden sicher. ${ }^{121}$

Die Bedingungen erster Ordnung für ein Nutzenmaximum lauten ${ }^{122}$

$$
\begin{array}{ll}
S: & u_{1, R}^{\prime}=(1+r)\left[\pi(h) u_{2 G, R}^{\prime}+(1-\pi(h)) u_{2 K, R}^{\prime}\right], \\
b: & u_{1, R}^{\prime}=\pi(h) \alpha(h) \beta_{R}^{\prime}(b) u_{2 G, R}^{\prime} .
\end{array}
$$

Bedingung (7.12) beschreibt den Zusammenhang zwischen den Grenznutzen der ersten und der zweiten Periode. Der Grenznutzen der ersten Periode $u_{1, R}^{\prime}$ muss

\footnotetext{
${ }^{121}$ In diesem Modell wird angenommen, dass die Zeit in der zweiten Periode entweder gesund oder aber erwerbsunfăhig verbracht wird. Ebenso wăre es denkbar, dass sich Erwerbsunfăhigkeit nur für einen Teil der zweiten Periode einstellt. In diesem Fall würde nicht mehr zwischen den unterschiedlichen Einkommenssituationen unterschieden werden, sondern das Einkommen in der zweiten Periode würde sich zu einem Anteil $\pi$ um das Erwerbseinkommen erhöhen und zu einem Anteil (1- $\pi$ ) um den Krankheitsschaden mindern, so dass sich das Problem (7.8) zu (7.4) reduziert. Eine Unterscheidung zwischen den Nutzen bei Gesundheit auf der einen und Krankheit auf der anderen Seite wäre nicht weiter möglich.

${ }^{122}$ Der Index $R$ kennzeichnet in diesem Abschnitt die Risikosituation mit positiver Erkrankungswahrscheinlichkeit, aber ohne Krankenversicherung.
} 
im Optimum den verzinsten erwarteten Grenznutzen der zweiten Periode im gesunden $\left(u_{2 G, R}^{\prime}\right)$ und im kranken Zustand $\left(u_{2 K, R}^{\prime}\right)$ entsprechen. Hieraus folgt, dass der Nutzenverzicht in der ersten Periode, der aus der Ersparnisbildung und damit der Minderung des für Konsumzwecke verfügbaren Einkommens entsteht, genau in Höhe des erwarteten Nutzenzuwachses in der zweiten Periode ausfallen muss. Aufgrund der unterstellten Konkavität der Nutzenfunktion liegt der Grenznutzen einer zusätzlichen Konsumeinheit bei Krankheit über dem Grenznutzen im gesunden Zustand, wie Abbildung 15 zeigt:

Abbildung 15: Abnehmender Grenznutzen des Konsums ${ }^{123}$

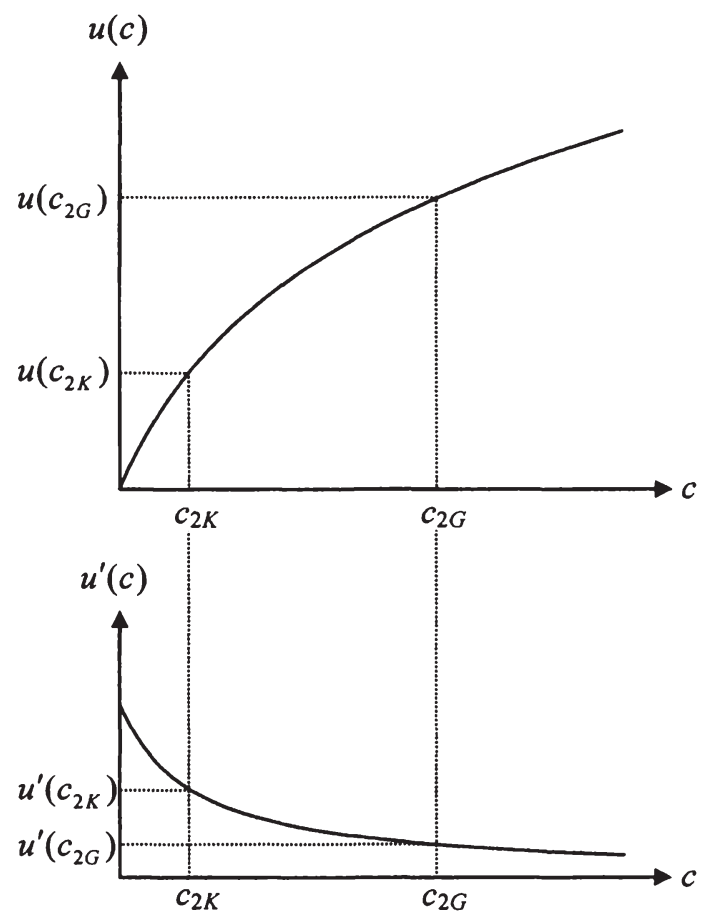

Quelle: Eigene Darstellung.

Je höher der Konsum ist, desto höher ist auch der Nutzen, den ein Individuum hierdurch erfährt. Die Nutzenzuwächse nehmen mit zunehmendem Konsum je-

123 Der hier gewählten Darstellung liegt eine Nutzenfunktion der Form $u(c)=\ln (c+1)$ zugrunde. Der Verlauf der Grenznutzenkurve wird damit durch die Funktion $u^{\prime}(c)=1 /(c+1)$ bestimmt. 
doch ab, weshalb der Nutzen des Konsums bei Gesundheit $\left(u\left(c_{2 G}\right)\right)$ zwar oberhalb des Nutzens bei Krankheit $\left(u\left(c_{2 K}\right)\right)$ liegt, der Grenznutzen im Krankheitsfall jedoch größer ist $\left(u^{\prime}\left(c_{2 K}\right)>u^{\prime}\left(c_{2 G}\right)\right)$.

Die zweite Bedingung (7.13) beschreibt den Zusammenhang zwischen den Kosten einer zusätzlichen Bildungseinheit und den erwarteten Erträgen. Die Nutzenminderung aufgrund der Bildungsbemühungen ist durch den Verzicht auf Erwerbseinkommen in der ersten Periode begründet. Dem steht ein erwarteter Nutzenzuwachs in der zweiten Periode aufgrund des erhöhten Erwerbseinkommens gegenüber. Dieser bestimmt sich über die Grenzproduktivität einer weiteren Zeiteinheit in der Bildungsproduktion sowie über die Wahrscheinlichkeit, in der zweiten Periode der Erwerbstätigkeit nachkommen zu können. Folglich müssen die Grenzkosten der Bildung (linke Seite) im Optimum den erwarteten Grenzerträgen (rechte Seite) entsprechen.

Unter Sicherheit über das zukünftige Arbeitsangebot werden die Erträge der Investitionen ausschließlich über die eingesetzte Zeit determiniert. Unter Unsicherheit sind darüber hinaus die Erwartungen darüber, ob die Erträge aus den Bildungsinvestitionen tatsächlich realisiert werden können oder ob sich ein Verlust aufgrund von Krankheit ergibt, bei der Entscheidung über den optimalen Bildungsumfang zu berücksichtigen (vgl. Snow und Warren (1990), S. 195). ${ }^{124}$

Für eine Bestimmung der optimalen Grenzproduktivität der Bildung werden zunächst die Bedingungen 1. Ordnung (7.12) und (7.13) jeweils nach $u_{1, R}^{\prime}$ umgeformt und anschließend gleichgesetzt. Auflösen nach der Grenzproduktivität ergibt

$$
\beta_{R}^{\prime}=\frac{1+r}{\alpha}\left[1+\frac{(1-\pi(h)) u_{2 K, R}^{\prime}}{\pi(h) u_{2 G, R}^{\prime}}\right] .
$$

Die optimale Grenzproduktivität der Bildung wird im Gegensatz zur Situation unter Sicherheit nicht mehr nur durch die Ersparnisse sowie die Lerneffizienz bestimmt (vgl. (7.7)), sondern auch durch die mit den Eintrittswahrscheinlichkeiten gewichteten Grenznutzen in den beiden Zuständen der zweiten Periode.

\footnotetext{
${ }^{124}$ Diese Überlegung ist insbesondere dann relevant, wenn es sich bei dem Arbeitsangebot nicht um eine Null-Eins-Entscheidung handelt wie in diesem Modell, sondern wenn eine längere zweite Erwerbsperiode betrachtet wird, in der nur ein Teil der Zeit gearbeitet wird, wăhrend das Individuum dem Arbeitsmarkt in der restlichen Zeit aufgrund von Krankheit nicht zur Verfügung steht.
} 
Da das Konsumniveau im kranken Zustand aufgrund des fehlenden Erwerbseinkommens sowie der zu finanzierenden Gesundheitsleistungen immer geringer ist als im gesunden Zustand, folgt hieraus unter der Annahme der Risikoaversion, dass der Grenznutzen bei Krankheit über demjenigen bei ausreichender Gesundheit liegen muss. Das Verhältnis der Grenznutzen ist damit größer Eins.

Kein Unterschied zur Grenzproduktivität unter Sicherheit würde sich ausschließlich dann ergeben, wenn ausreichende Gesundheit zu einem sicheren Ereignis wird, d. h. für $\pi(h)=1$. In diesem Fall ist das Verhältnis der erwarteten Grenznutzen gleich Null, während Gleichung (7.14) für eine Wahrscheinlichkeit von Null nicht definiert ist. Für alle übrigen Situationen mit $0<\pi(h)<1$ ist der Wert der eckigen Klammer immer größer Eins, denn das Verhältnis der erwarteten Grenznutzen ist größer Null, sobald eine positive Erkrankungswahrscheinlichkeit gegeben ist. Damit ist die Grenzproduktivität der Bildung in diesen Situationen stets höher als im Fall absoluter Sicherheit. Aufgrund der unterstellten Konkavität der Bildungsproduktionsfunktion folgt hieraus ein geringerer Zeitaufwand für Bildungszwecke $b$.

Je wahrscheinlicher eine zweite Erwerbsperiode ist, desto kleiner ist der Quotient aus den erwarteten Grenznutzen und damit c. p. die rechte Seite von (7.14). Hieraus ergibt sich eine steigende Bereitschaft, Zeit in Bildung $\mathrm{zu}$ investieren und darüber im gesunden Zustand der zweiten Periode zusätzliches Einkommen zu erzielen, da die Unsicherheit über die Erträge der Bildungsinvestition sinkt. Je weiter hingegen die Konsumniveaus der beiden Zustände voneinander entfernt sind, desto größer wird die rechte Seite von Gleichung (7.14) und desto geringer ist der Anreiz, in Bildung zu investieren.

Im Vergleich mit der Bildungssituation unter Sicherheit zeigt sich, dass ein positives Krankheitsrisiko ohne Krankenversicherungsschutz dazu führt, dass weniger Zeit in die Bildung investiert wird. Umformen von (7.14) nach dem Grenznutzenverhältnis ergibt

$$
\frac{u_{2 K, R}^{\prime}}{u_{2 G, R}^{\prime}}=\frac{\pi(h)}{1-\pi(h)}\left[\frac{\alpha(h) \beta_{R}^{\prime}}{1+r}-1\right]>1 .
$$

Damit Bedingung (7.15) erfüllt ist, muss die erwartete Grenzproduktivität der Bildung größer sein als die Verzinsung, $d$. $h$.

$$
\pi \alpha \beta_{R}^{\prime}>1+r .
$$


Analog zur Situation unter Sicherheit spielt der Zins eine wesentliche Rolle bei der Bestimmung der optimalen Zeitallokation unter Unsicherheit. Je höher der Kapitalmarktzins ist, desto geringer ist der Anreiz, Zeit in die Bildung zu investieren, da die sicheren Erträge einer Kapitalinvestition ansteigen, die Erträge der Bildungsinvestitionen jedoch weiter unsicher sind. Damit muss der erwartete Grenzertrag der Bildung im Optimum größer sein als die Verzinsung des Realkapitals. Liegt also der erwartete Grenzertrag der Bildung über der Verzinsung, werden Ressourcen vom physischen Kapital hin zum Bildungskapital realloziert, bis Bedingung (7.16) nicht weiter erfüllt werden kann. Hieraus folgt für die Investitionen in die Bildung unter Verwendung einer identischen, $d . h$. unveränderten Bildungsproduktionsfunktion:

$$
\pi \beta_{R}^{\prime}>\beta^{\prime} \Leftrightarrow b_{R}<b
$$

Diese Bedingung ist für jede Unsicherheitssituation $\pi(h)<1$ erfült, wenn die Grenzproduktivität der Bildung ohne Krankenversicherung die erreichte Grenzproduktivität unter Sicherheit übersteigt. Je höher die Wahrscheinlichkeit einer Erkrankung ist, d. h. je niedriger $\pi(h)$ ist, desto höher muss die Grenzproduktivität bei Unsicherheit sein, damit die Bedingung (7.17) erfullt ist. Aufgrund der Annahme abnehmender Grenzerträge der Bildungsinvestitionen ergibt sich hieraus, dass umso weniger Zeit für Bildungszwecke aufgewendet wird, je wahrscheinlicher Arbeitsunfähigkeit in der zweiten Periode eintritt. Vielmehr besteht der Anreiz, die in der ersten Periode verfügbare Zeit in Arbeit zu investieren, um einen Teil des Konsums sicher in die zweite Periode transferieren zu können. ${ }^{125}$

Abbildung 16 zeigt den Zusammenhang zwischen Bildungsinvestitionen und Ersparnissen für diese Unsicherheitssituation anhand eines 5-Felder-Diagramms, wobei nicht mehr die Ersparnissumme der ersten Periode, sondern die Sparquote $s$ betrachtet wird. ${ }^{126}$ Die Kurve im I. Quadranten entspricht einer Konsummöglichkeitenkurve in der ersten Periode, die die Sparquote zu der Bildungszeit $b$ in Beziehung setzt. Unter der Annahme, dass das Konsumniveau der ersten Periode konstant ist, bestimmt sich die Grenzrate der Substitution durch $\mathrm{d} s / \mathrm{d} b=-(1-s) /(1-b)$. Der konkave Verlauf wird folglich durch das negative Verhältnis aus Konsumquote und Arbeitszeit bestimmt. Je höher die Konsumquote ausfällt, desto weniger wird für die Zukunft gespart und desto flacher ist

\footnotetext{
${ }^{125}$ Brunello (2002) untersucht den Einfluss von Risikoaversion auf die Bildungsentscheidung vor dem Hintergrund unsicherer Bildungserträge. Besteht keine Möglichkeit, sich gegen diese Unsicherheit zu versichern, fuhrt absolute Risikoaversion dazu, dass weniger Zeit in die Bildung investiert wird.

${ }^{126}$ Gleichung (7.9) wird dann $\mathrm{zu} c_{1}=(1-s)(1-b) w$.
} 
der Verlauf der Konsummöglichkeitenkurve. Das Verhältnis aus Konsum und Lohnsatz bestimmt hingegen die Lage der Kurve im I. Quadranten. Ein hoher Lohnsatz ermöglicht c. p. einen hohen Bildungszeitanteil, während ein hohes Konsumniveau in der ersten Periode den maximal möglichen Bildungszeitaufwand senkt.

Abbildung 16: Investitionsentscheidung ohne Krankenversicherung

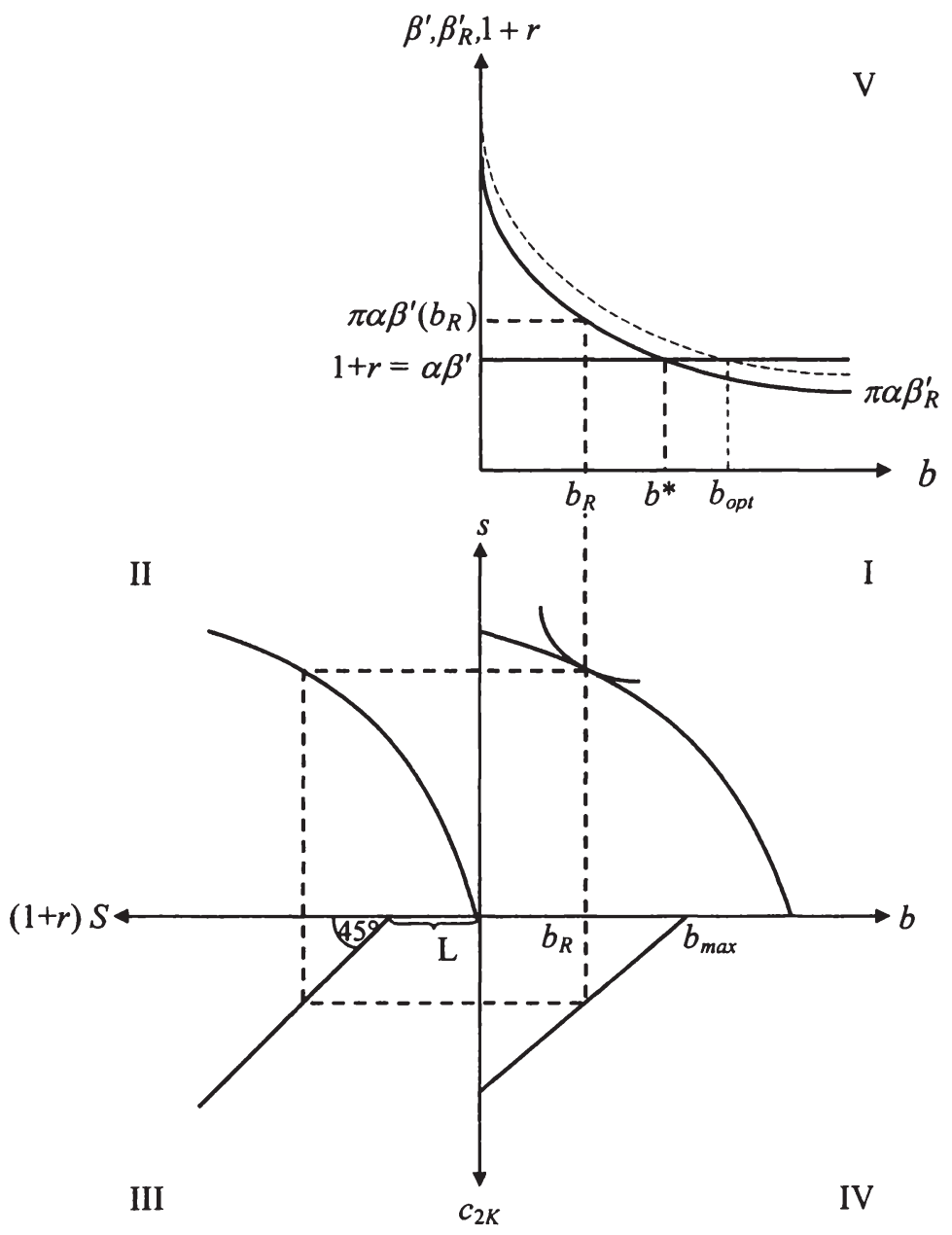

Quelle: Eigene Darstellung. 
Der II. Quadrant beschreibt den Zusammenhang zwischen der Sparquote und den verzinsten Ersparnissen. Um den Zusammenhang abzubilden, wird $b \mathrm{zu}-$ nächst gegen $s$ substituiert, so dass die Ersparnisbildung durch die Gleichung

$$
S=\frac{s}{1-s} c_{1}
$$

darstellen lässt. Hieraus ergibt sich für die Abhängigkeit der verzinsten Ersparnisse von der Sparquote:

$$
\frac{d(1+r) S}{d s}=\frac{(1+r) c_{1}}{(1-s)^{2}}
$$

Der Kurvenverlauf ist damit umso steiler, je größer das Verhältnis aus dem tatsächlichen Konsumniveau und der quadrierten Konsumquote ist. Je höher zudem der Zinssatz ist, desto höher sind die Erträge der Ersparnisse und desto größer ist entsprechend die Steigung der Kurve.

Im III. Quadranten ist die Relation zwischen den verzinsten Ersparnissen und dem Konsum bei Krankheit in der zweiten Periode abgebildet, wobei annahmegemäß keine weiteren Einkünfte zur Verfügung stehen. Der Abszissenabschnitt wird durch den Krankheitsschaden $L$ festgelegt.

Aus diesen drei Quadranten leitet sich ein negativer Zusammenhang zwischen den Bildungsinvestitionen in der ersten Periode und dem Konsumniveau der zweiten Periode, dargestellt im IV. Quadranten, ab. Hier ergibt sich ebenfalls ein linearer Zusammenhang, da bei konstantem Konsum in Periode 1 sämtliche zusätzlichen Einkünfte, die aus einer Erhöhung der Arbeitszeit resultieren, direkt über die Ersparnisse in die zweite Periode transferiert werden. ${ }^{127} \mathrm{Je}$ höher der Zins sowie der Ursprungslohnsatz sind, desto flacher ist der Verlauf der Geraden, da der Verzicht auf eine Bildungseinheit den Konsum bei Krankheit umso stärker ansteigen lässt. Der Ordinatenabschnitt $\left(c_{2 K}\right)$ wird bei gegebenem Konsumniveau in der ersten Periode durch die maximale Sparquote bestimmt. Der Abszissenabschnitt und damit der für die Bildung maximal mögliche Zeitaufwand $b_{\max }$ liegt zudem umso dichter am Ursprung, je größer der erwartete Krankheitsschaden $L$ ist, da Verschuldung nicht zugelassen ist und die Ersparnisse ausreichen müssen, um die mit Krankheit verbundene finanzielle Belastung zu decken. Je nach Erkrankungswahrscheinlichkeit und Risikopräferenz

${ }^{127}$ Unter der Annahme des konstanten Konsums $c_{1}$ beträgt die Steigung $\frac{d b}{d c_{2 K}}=-\frac{1}{(1+r)_{w}}$. 
liegt die gewählte Bildungszeit zwischen dem Ursprung und $b_{\max }$. In diesem Beispiel wird die Bildungszeit $b_{R}$ gewählt.

Der V. Quadrant bildet erneut die Grenzerträge beider Kapitalarten bei gegebener Lerneffizienz ab. Der Grenzertrag der Ersparnisse ist nach wie vor konstant, die Grenzproduktivität der Bildung mit zunehmendem Zeitaufwand abnehmend. Aufgrund der Tatsache, dass es sich hierbei um eine Unsicherheitssituation handelt, wird die Grenzproduktivität der Bildung gemäß Bedingung (7.16) mit der Wahrscheinlichkeit, gesund zu bleiben, $\pi(h)$, gewichtet. Die Bildungskurve verschiebt sich entsprechend Richtung Ursprung und bildet jetzt die erwarteten Grenzerträge der Bildungsinvestitionen ab.

Der Schnittpunkt dieser beiden Kurven $\left(b^{*}\right)$, in dem sich die Grenzerträge beider Kapitalarten entsprechen würden, liegt jetzt links des alten Optimums $b_{o p t}$. Aus Bedingung (7.16) folgt darüber hinaus, dass die Grenzproduktivität der Bildung bei Unsicherheit für jede positive Erkrankungswahrscheinlichkeit größer sein muss als die Verzinsung, so dass das Optimum im Risikofall $b_{R}$ in jedem Fall links vom Bildungsaufwand im Schnittpunkt $b^{*}$ liegt; andernfalls wäre Bedingung (7.15) nicht erfüllt, der Konsum bei Krankheit wäre größer als der Konsum bei Gesundheit. Je weiter sich der tatsächliche Zeitaufwand $b_{R}$ vom Gleichgewicht in Richtung Ursprung entfernt, desto höher ist das gewünschte Konsumniveau bei Krankheit in der zweiten Periode und desto höher ist damit die Risikoaversion des einzelnen Individuums. ${ }^{128}$

Sobald sich eine Veränderung der Gesundheit einstellt, ändern sich damit gleichzeitig die Rahmenbedingungen für Bildungsinvestitionen. Gesundheitsveränderungen sind im Modell zwar exogen bestimmt, d. h. bewusste Investitionen sind nicht möglich, nichts desto trotz sind derartige Veränderungen beobachtbar. So geht eine Verbesserung des individuellen Gesundheitszustandes mit einer geringeren Erkrankungswahrscheinlichkeit sowie einer gesteigerten Lerneffizienz einher, weshalb die erwartete Rendite der Investitionen ansteigt und so mehr Zeit in Bildung investiert werden kann. In der obigen Abbildung würde sich die Kurve der Bildungsgrenzproduktivität bei einer Verbesserung der Ge-

\footnotetext{
${ }^{128}$ In dem hier angeführten Fall liegt der für Bildungszwecke maximal mögliche Zeitaufwand rechts vom Gleichgewicht $b^{\circ}$. Ist die Verzinsung im Verhältnis zur Grenzproduktivität der Bildung sehr hoch oder sind für eine Deckung des Krankheitsschaden hohe Ersparnisse notwendig, so ist es jedoch auch möglich, dass die maximale Investitionszeit links vom Schnittpunkt $b^{*}$ im 5. Quadranten liegt.
} 
sundheit nach rechts oben verschieben, wodurch mehr Bildung nachgefragt werden könnte, ohne die Optimalitätsbedingungen zu verletzen.

Für die formale Abhängigkeit der Bildungsinvestitionen vom individuellen Gesundheitszustand folgt unter Anwendung des impliziten Funktionentheorems aus (7.13):

$$
\frac{d b_{R}}{d h}=-\frac{\beta_{R}^{\prime}\left[\pi^{\prime} \alpha+\pi \alpha^{\prime}\right] u_{2 G, R}^{\prime}+\pi^{\prime}(1+r) U_{R}^{\prime}+\Omega_{R} u_{2 G, R}^{\prime \prime}}{\pi \alpha \beta_{R}^{\prime \prime} u_{2 G, R}^{\prime}+\pi\left[\alpha \beta_{R}^{\prime}-(1+r)\right] \alpha \beta_{R}^{\prime} w u_{2 G, R}^{\prime \prime}},
$$

wobei

$$
U_{R}^{\prime} \equiv u_{2 K, R}^{\prime}-u_{2 G, R}^{\prime}
$$

und

$$
\Omega_{R} \equiv \pi\left[\alpha \beta_{R}^{\prime}-(1+r)\right] \alpha^{\prime} \beta_{R} w>0
$$

Der Nenner in (7.18) ist aufgrund der unterstellten Konkavität der Nutzen- sowie der Bildungsproduktionsfunktion negativ, so dass sich für einen positiven Zähler eine Ausweitung der Bildung aufgrund einer Verbesserung der Gesundheit ergibt. Insgesamt lässt sich der Einfluss einer verbesserten Gesundheit auf die Bildungsinvestitionen in vier Partialeffekte untergliedern. Der Term $\pi^{\prime} \alpha$ kann als Sicherheitseffekt interpretiert werden: Aufgrund der Verbesserung der Gesundheit steigt die Wahrscheinlichkeit, dass die Bildungserträge in der zweiten Periode tatsächlich realisiert werden können. Dieser Effekt wirkt sich um so stärker positiv auf die Investitionen in die Bildung aus, je höher die Lerneffizienz ist, da dann eine zusätzliche Bildungseinheit um so größere Einkommenssteigerungen mit sich bringt.

Der Ausdruck $\pi \alpha^{\prime}$ beschreibt die positive Wirkung der Gesundheit auf die Lerneffizienz und kann somit als Effizienzeffekt umschrieben werden. Dieser Effekt ist positiv von der Erwerbswahrscheinlichkeit abhängig, da sich dann die

\footnotetext{
${ }^{129}$ Eine derartige Verschiebung würde die maximale Bildungszeit zwar erhöhen, diese würde jedoch nach wie vor unterhalb des Zeitaufwands bei Sicherheit liegen, da sich die erwartete Grenzproduktivităt lediglich an die Grenzproduktivität annăhert, diese aber für jedes $\pi<1$ nicht erreicht (siehe Gleichung (7.17)).
} 
Zuwächse in der Lerneffizienz umso wahrscheinlicher auf das Konsumniveau in der zweiten Periode auswirken. Für beide Effekte gilt, dass diese umso stärker ausgeprägt sind, je weniger Zeit ohne eine Verbesserung der Gesundheit in die Bildung investiert wird und je geringer damit das Erwerbseinkommen in der zweiten Periode ausfällt.

Der dritte Einflussfaktor enthält den Term $\pi^{\prime}(1+r)$ und lässt sich daher als Zinseffekt charakterisieren. Dieser führt ebenfalls zu einer gesteigerten Akkumulation von Bildungskapital, denn mit steigender Erwerbswahrscheinlichkeit geht die Bedeutung der sicheren Ersparnisse für den Konsum zurück. Ein hoher Zins kann diesen Zinseffekt verstärken, da mit wenig Ersparnis ein relativ hohes Zinseinkommen erzielt werden kann, so dass der Einkommensrückgang bei Krankheit leichter zu kompensieren ist. Zudem nimmt die Bedeutung der Ersparnisbildung immer weiter $a b$, je deutlicher sich eine Verbesserung der Gesundheit auf die Erwerbswahrscheinlichkeit auswirkt.

Negativ wirkt sich lediglich der Substitutionseffekt $\Omega_{R} u_{2 G, R}^{\prime \prime}$ auf die Bildungsinvestitionen aus, denn bei gesteigerter Lerneffizienz kann das gleiche Bildungsniveau mit weniger Zeitaufwand erreicht werden. Mit Hilfe der freigesetzten Zeit kann das Erwerbseinkommen in der ersten Periode gesteigert und in Form von zusätzlichen sicheren Ersparnissen in die zweite Periode transferiert werden. Bei einer hohen Wahrscheinlichkeit einer zweiten Erwerbsperiode können die Effizienzgewinne $\alpha^{\prime}$ eher realisiert werden, so dass bei weniger Bildung nicht auf Einkommen verzichtet werden muss.

Ob sich eine Verbesserung der Gesundheit letztlich positiv oder negativ auf die Bildungsentscheidung auswirkt, hängt maßgeblich von der Risikoaversion des Individuums ab. Bei hoher Risikoaversion wird das Einkommen bei Krankheit stärker gewichtet, so dass auch bei sehr geringer Erkrankungswahrscheinlichkeit eine hohe Sparneigung besteht. In diesem Fall kann der Substitutionseffekt die übrigen Partialeffekte überwiegen, die Bildungsinvestitionen würden bei einer Verbesserung der Gesundheit abnehmen. Je geringer die Risikoaversion hingegen ist, desto geringer ist auch die Bedeutung des Substitutionseffektes, es kommt zu einem positiven Einfluss der Gesundheit auf die Bildung.

Aus diesem Modell der Bildungsinvestitionen unter Unsicherheit lassen sich folgende Implikationen ableiten: Es zeigt sich, dass die beiden Humankapitalarten Bildung und Gesundheit nicht unabhängig voneinander betrachtet werden können, sondern dass Interdependenzen existieren. Soll der Bildungskapitalbestand innerhalb einer Ökonomie erhöht werden, kann dies zum einen durch eine Verbesserung der Bildungsinfrastruktur geschehen, um eine gesteigerte Bildungsproduktivität zu ereichen. Zum anderen besteht die Möglichkeit, eine 
Verbesserung der individuellen Gesundheit zu erreichen, indem beispielsweise mehr Gesundheitsleistungen verfügbar sind, so dass sich hieraus ein positiver Anreiz zu mehr Bildungsinvestitionen ergibt. ${ }^{130}$

Eine Möglichkeit, die Unsicherheit über das Einkommen bei Krankheit zu begrenzen, besteht in der Einführung einer Krankenversicherung. Wenn diese dazu beitragen kann, das Vorsichtsmotiv bei der Bildungsentscheidung abzuschwächen, indem das Einkommen bei Krankheit abgesichert wird, kann die Verbesserung der Gesundheit insbesondere von Kindern und Jugendlichen, die sich noch in der Bildungsperiode befinden, einen effizienten Weg darstellen, die Beschulung per se sowie deren Erfolg zu verbessern (vgl. Behrman (1996), S. 33).

\subsection{Einführung einer prämienfinanzierten Krankenversicherung}

In Abschnitt 7.2 kann ein positiver Konsum bei Krankheit ausschließlich über die Ersparnisse erreicht werden, so dass diesen eine existentielle Bedeutung beigemessen wurde. Eine weitere Möglichkeit, Bildungsinvestitionen zu fördern, besteht in der Einführung einer Krankenversicherung. Krankenversicherungsschutz senkt erstens die Unsicherheit hinsichtlich des Konsums in der zweiten Periode, indem zumindest ein Teil des bei Krankheit auftretenden finanziellen Schadens $L$ durch die Versicherung übernommen wird. Zweitens besteht darluber hinaus die Möglichkeit, Lohnersatzleistungen in Form von Krankengeldzahlungen zu leisten, um das Einkommensrisiko weiter abzuschwächen. Somit erfolgt durch die Versicherung eine Umverteilung zwischen den beiden Zuständen der zweiten Periode.

In dem nachfolgenden Modell wird die Versicherung über eine Prämie $P$ finanziert, die sowohl bei Gesundheit als auch bei Krankheit in der zweiten Periode zu entrichten ist. Diese Prämie wird pro Kopf erhoben und ist damit unabhängig vom individuellen Erkrankungsrisiko sowie vom verfügbaren Einkommen. Grundsätzlich ist es in einem solchen Prämiensystem unerheblich, aus welchem Teil des Einkommens die Zahlungen geleistet werden, so dass Erwerbs- und Zinseinkünfte gleich behandelt werden. Die Versicherungsleistung im Krankheitsfall erfolgt über Lohnersatzleistungen, die sich proportional am Lohn der ersten Periode ausrichten, sowie über einen finanziellen Ausgleich für medizinische Leistungen. Diese Modifikation gegenüber dem Krankenversicherungssystem aus Kapitel 5 ist notwendig, da Lohnersatzleistungen für das Vorsichtssparmotiv wesentlich und daher bei der Optimierung zu berücksichtigen sind.

\footnotetext{
${ }^{130}$ Aus Vereinfachungsgründen wurde im Rahmen der hier gewăhlten Modelldarstellung von einem gesundheitsfordernden Einfluss der Bildung auf die Gesundheit abstrahiert. An dieser Stelle sei daher auf Kapitel 4.4 verwiesen.
} 
Beschreibt nun $P_{1}$ die zu leistende Prämie in der ersten und $P_{2}$ die Prämie der zweiten Periode, dann ergibt sich folgendes Maximierungsproblem für ein Prämiensystem:

$$
\max _{S, b} u\left(c_{1}\right)+\pi(h) u\left(c_{2 G}\right)+[1-\pi(h)] u\left(c_{2 K}\right)
$$

unter den Nebenbedingungen

$$
\begin{aligned}
& c_{1}=(1-b) w-S-P_{1}, \\
& c_{2 G}=(1+r) S+[1+\varphi] w-P_{2}, \\
& c_{2 K}=(1+r) S+q w-(1-v) L-P_{2}, \\
& \sum_{i}\left(1-\pi_{i}\right)\left[v L_{i}+q w\right]=\sum_{i} P_{2, i}
\end{aligned}
$$

Die ersten drei Nebenbedingungen (7.21)-(7.23) zeigen erneut die Konsumniveaus für die einzelnen Zustände. Der Konsum in der ersten sowie im gesunden Zustand der zweiten Periode verändert sich gegenüber der Situation ohne Krankenversicherung (siehe (7.9) bzw. (7.10)) um die jeweiligen Prämienzahlungen.

Für den Konsum im Krankheitsfall, dargestellt in Gleichung (7.23), steht ein Einkommen aus den verzinsten Ersparnissen sowie aus den Lohnersatzleistungen zur Verfügung. Letztere werden durch die Lohnersatzquote $q \in[0,1]$ bestimmt. Zudem erhält das Individuum einen finanziellen Ausgleich für seine Ausgaben für medizinische Leistungen in Höhe von $v L$, wobei $v$ der Höhe des Deckungssatzes entspricht mit $v \in[0,1]$. Für $v=1$ werden sämtliche Kosten durch die Krankenversicherung übernommen, so dass sich der Konsum in der zweiten, krank verbrachten Periode auf die verzinsten Ersparnisse zuzüglich der Lohnersatzleistungen beläuft. In diesem Fall entstehen dem Individuum durch den Gesundheitsschaden lediglich Opportunitätskosten in Höhe der Differenz aus dem entgangenen Erwerbseinkommen und den Lohnersatzleistungen.

Direkte Kosten sind mit einer Krankheit nicht verbunden, wenn die Leistungen der Krankenversicherung ohne Restriktionen in Anspruch genommen werden können. Beträgt der Deckungssatz der Versicherung hingegen Null, beschränkt sich die Versicherungsleistung auf die Zahlung von Lohnersatzleistungen. Für jeden beliebigen Deckungsanteil $0<v<1$ hat das Individuum dann einen 
Selbstbeteiligungssatz in Höhe von (1-v) der Schadenssumme als Eigenanteil zu übernehmen.

Als weitere Belastung kommt in jedem Fall die Zahlung der Prämie $P_{2}$ in vollem Umfang hinzu. Da Verschuldung in der zweiten Periode erneut nicht zulässig ist, muss die Summe aus den verzinsten Ersparnissen sowie den Ersatzleistungen den privat $\mathrm{zu}$ finanzierenden Schadensanteil sowie die Prämienzahlungen zumindest decken, d. h. $(1+r) S+q w \geq(1-v) L-P_{2}$.

Gleichung (7.24) zeigt die Nullgewinnbedingung für die Krankenversicherung in Periode 2. Auf der linken Seite ist die Summe der erwarteten Ausgaben der Versicherung für alle Individuen $i$ aufgeführt, die den Gesamteinnahmen aus den Prämienzahlungen, dargestellt auf der rechten Seite, entsprechen müssen. Dieser Gleichung liegt die Prämisse zugrunde, dass sich die Versicherung über ein Umlageverfahren, das an das System der deutschen GKV angelehnt ist, finanziert. Während die Ausgaben der Versicherung pro Periode von der Erkrankungswahrscheinlichkeit abhängen und damit der Unsicherheit unterliegen, sind die Einnahmen sicher. Je nach Erkrankungswahrscheinlichkeit der Individuen einer Generation können sich hieraus Prämiensteigerungen oder -senkungen für nachfolgende Generationen ergeben.

Aufgrund der fehlenden Einkommensbezogenheit hat die individuelle Entscheidung uber die Zusammensetzung des Einkommens in der zweiten Periode keinerlei Auswirkungen auf die Einnahmeseite der Versicherung, so dass diese nicht in das Kalkül der Individuen einbezogen wird. Nachfolgend wird weiterhin angenommen, dass den Individuen im Hinblick auf die Versicherungsbedingungen keine Wahlmöglichkeit verbleibt. Die Lohnersatzquote, der Deckungssatz und damit auch die Prämienhöhe sind exogen vorgegeben. Wird zudem angenommen, dass Investitionen in die Gesundheit nach wie vor nicht möglich sind, bleiben die Ersparnisse $S$ sowie die Bildungsbemühungen $b$ die einzigen Entscheidungsvariablen. Damit lauten die Bedingungen erster Ordnung für ein Nutzenmaximum $^{131}$ :

$$
\begin{array}{ll}
S: & u_{1, P}^{\prime}=(1+r)\left[\pi(h) u_{2 G, P}^{\prime}+(1-\pi(h)) u_{2 K, P}^{\prime}\right], \\
b: & u_{1, P}^{\prime}=\pi(h) \alpha(h) \beta_{P}^{\prime}(b) u_{2 G, P}^{\prime} .
\end{array}
$$

${ }^{131}$ Zum Zweck der besseren Vergleichbarkeit kennzeichnet der Index P die Modellvariablen bei einer prämienfinanzierten Krankenversicherung. 
Durch Gleichsetzen der Bedingungen (7.25) und (7.26) und Umformen erhält man für die optimale Grenzproduktivität der Bildung

$$
\beta_{P}^{\prime}=\frac{1+r}{\alpha}\left[1+\frac{(1-\pi(h)) u_{2 K, P}^{\prime}}{\pi(h) u_{2 G, P}^{\prime}}\right]
$$

Erneut gilt für das Verhältnis der Grenznutzen, dass dieses größer Eins sein muss, da aus der Krankheit in Verbindung mit der Versicherung kein finanzieller Vorteil entstehen darf (vgl. Gleichung (7.14)). Dieser Zusammenhang ist für alle Ersatzquoten $q \leq 1$ gegeben, so dass die Lohnersatzleistungen maximal in Höhe des Ursprungslohnsatzes geleistet werden. Damit folgt für die erwartete Grenzproduktivität, dass diese erneut größer sein muss als die Verzinsung:

$$
\pi \alpha \beta_{P}^{\prime}>1+r .
$$

Diese Bedingung entspricht der Optimalitätsbedingung ohne Versicherung in (7.16), es entstehen folglich keine Verzerrungen durch die Prämienzahlungen, da diese in beiden Perioden gleichermaßen zu leisten sind.

Der für die Bildung aufgewendete Zeitanteil $b$ ist gegenüber der Unsicherheitssituation genau dann höher, wenn das Verhältnis der Grenznutzen kleiner ist als unter Unsicherheit, so dass $u_{2 K, P}^{\prime} / u_{2 G, P}^{\prime}<u_{2 K, R}^{\prime} / u_{2 G, R}^{\prime}$ gelten muss. Diese Bedingung ist erfüllt, sobald die Versicherung eine Konsumglättung zwischen den Zuständen zur Folge hat, denn dann liegt der Konsum im gesunden Zustand unter dem Konsumniveau ohne Versicherung, während gleichzeitig der Konsum bei Krankheit das Niveau bei Unsicherheit übersteigt. Da eine Versicherung stets eine Senkung des Risikos aufgrund einer Umverteilung der Einkommen zum Ziel hat, bietet die Einführung einer prämienfinanzierten Krankenversicherung generell den Anreiz, vermehrt Zeit in die Bildung zu investieren. Die tatsächliche Höhe der Prämie spielt nur indirekt über die Beeinflussung der Grenznutzen eine Rolle, da diese sowohl aus Kapital- als auch aus Erwerbseinkommen beglichen werden kann. Folglich ergeben sich in einem Prämiensystem keine Verzerrungen auf die Bildungsinvestitionen.

Diese Zusammenhänge können ebenfalls grafisch dargestellt werden. In Abbildung 17 wird angenommen, dass sich das Konsumniveau in der ersten Periode nicht verändert. Die finanziellen Einbußen aufgrund der Prämienzahlungen führen entsprechend dazu, dass die neue Konsummöglichkeitenkurve im I. Quadranten unterhalb der Kurve ohne Versicherung verläuft. Darüber hinaus 
sind die Spar- und die Bildungsquote nicht mehr proportional zueinander, die neue Grenzrate der Substitution lautet

$$
\frac{d s}{d b}=-\frac{(1-s) w}{(1-b) w-R}
$$

und wird daher durch die Prämienhöhe mit beeinflusst. ${ }^{132}$ Zusätzlich zu der Steigung bestimmt die Prämie auch die Lage der neuen Konsummöglichkeitenkurve. Zuletzt legt die Prämienhöhe gleichzeitig den Möglichkeitsraum für Spar-. und Bildungsmaßnahmen fest. Unter der Annahme eines konstanten Konsumniveaus in der ersten Periode geht die maximal mögliche Bildungsquote stärker zurück als die Sparquote, da das Erwerbseinkommen der ersten Periode aufgrund der zu finanzierenden Prämienzahlungen an Bedeutung gewinnt.

Der Zinssatz bleibt auch nach Einführung der Versicherung unverändert, so dass die Zinskurve im II. Quadranten genau derjenigen im Fall ohne Versicherung entspricht. ${ }^{133}$ Damit wird allen möglichen Sparquoten die gleiche verzinste Ersparnis zugeordnet, während der für Bildung verfügbare Zeitanteil für alle Sparquoten geringer ausfällt.

Nun ist die Annahme, dass sich der Konsum der ersten Periode trotz höherer finanzieller Belastung nicht verändert, sehr restriktiv. Durch die Einführung der Prämie sinken c. p. die verfügbaren Einkommen beider Perioden. Damit ist es ebenso möglich, dass ein Teil des Konsums der ersten Periode in die zweite Periode transferiert wird, um finanzielle Einbußen auszugleichen. Wird in der ersten Periode nach Einführung der Prämie weniger konsumiert, verläuft die Kurve im zweiten Quadranten flacher. Bei gleicher Sparquote wird damit weniger verzinstes Kapitaleinkommen erzielt.

Wie sich der Konsum der ersten Periode tatsächlich verändert, hängt nicht zuletzt von dem bis dato erreichten Konsumniveau ab. Je niedriger dieses ohne Prämie bereits ist, desto unwahrscheinlicher werden Substitutionseffekte zugunsten des zukünftigen Konsums. Im Rahmen der in Abbildung 17 gewählten Darstellung wird nun zum Zweck der Vergleichbarkeit angenommen, dass das Konsumniveau $c_{1}$ unverändert bleibt.

${ }^{132}(7.21)$ wird zu $c_{1}=(1-s)\left[(1-b) w-P_{1}\right]$.

133 Die Steigung der Kurve hängt erneut vom Zins, dem Konsumniveau der ersten Periode sowie der Sparquote selbst ab (siehe hierzu S. 136). 
Abbildung 17: Bildungsinvestitionen bei prämienfinanziertem Krankenversicherungsschutz und unverändertem Konsumniveau

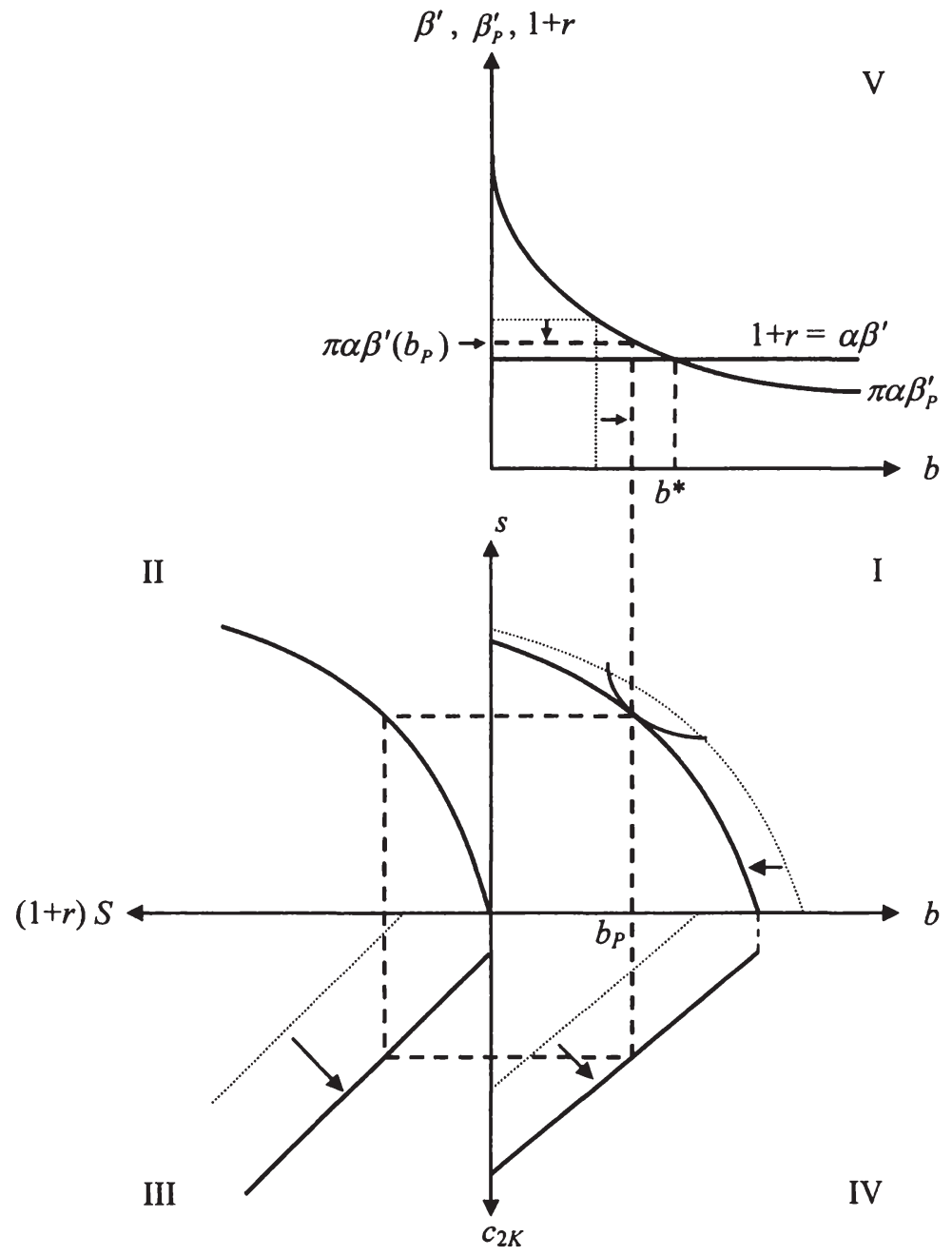

Quelle: Eigene Darstellung.

Im III. Quadranten ist erneut die Relation zwischen Ersparnis und Konsum bei Krankheit abgebildet. An dieser Stelle wird davon ausgegangen, dass die Versicherungsleistung ausreicht, um die Krankheitskosten vollständig abzudecken sowie ein finanzielles Minimum auch ohne Ersparnis zu garantieren $(v=1$, 
$q>0$ ). Somit bleibt trotz der Einschränkungen in der ersten Periode mehr Spielraum für Bildungsinvestitionen, da die Ersparnisrestriktion wegfällt. Dementsprechend verschiebt sich auch die Konsumkurve im IV. Quadranten parallel nach außen. Würde die Bildungsquote bis zum Maximum ausgeweitet, so dass keine Ersparnisse gebildet würden, stünde dennoch ein Basiseinkommen bei Krankheit zur Verfügung, so dass das Verlustrisiko bei Krankheit abgemindert wird. Dieser Mindestkonsum ist durch den senkrechten Bereich der Konsumkurve im IV. Quadranten gekennzeichnet.

Unter der Annahme, dass zwar weiterhin ein Teil des Einkommens der ersten Periode gespart wird, insgesamt aufgrund der Versicherungsleistung in der zweiten Periode aber weniger Ersparnisse erforderlich sind, kann der Konsum der ersten Periode aus einem geringeren Erwerbseinkommen gedeckt werden. Folglich steigt der optimale Bildungszeitaufwand im Vergleich zur Situation ohne Krankenversicherung an. Das neue Optimum im IV. Quadranten liegt bei $b_{P}$ und damit rechts von der Situation ohne Versicherung.

Versicherungsschutz kann somit dazu führen, dass bei gleichem Konsumniveau in der ersten Periode mehr Bildungskapital zu Lasten der Ersparnisse gebildet wird. Anhand des V. Quadranten wird deutlich, dass eine solche Entwicklung möglich ist, ohne die Optimalitätsbedingung zu verletzen. Der Zinssatz sowie die erwartete Grenzproduktivität bleiben im Vergleich zu Abbildung 16 unverändert, der Zeitaufwand für Bildungszwecke verschiebt sich jedoch von $b_{R}$ nach rechts, bis der Zeitanteil $b_{P}$ erreicht ist. Während die Prämie die Lage der Kurven in den Quadranten I und IV mitbestimmt, wirkt sich eine Anhebung oder Senkung der Prämie nicht weiter auf die Kurvenverläufe im V. Quadranten aus, so dass der Gleichgewichtspunkt $b^{*}$ unabhängig von den Entwicklungen im Gesundheitssystem ist. Damit werden Bildungsinvestitionen umso stärker gefördert, je mehr das Vorsorgesparen an Bedeutung verliert.

Da die Einführung einer Krankenversicherung Bildungsinvestitionen begünstigt, stellt sich hier die Frage, ob eine Verbesserung der Gesundheit nach wie vor einen positiven Einfluss auf die Bildung ausüben kann. Dadurch, dass sich im Hinblick auf die Bildungsbereitschaft keine Verzerrungen aus der Erhebung der Prämie ergeben, sind die Bedingungen erster Ordnung im Vergleich zu der Unsicherheitssituation ohne Versicherung unverändert, die Bedeutung der Gesundheit wird erneut durch Gleichung (7.18) abgebildet. Unterschiede ergeben sich hier lediglich in der Stärke der einzelnen Partialeffekte, die durch den Verlauf der Nutzenfunktion bestimmt werden. Die Richtung der einzelnen Effekte bleibt erhalten, während sich die Ausprägung aufgrund einer Veränderung der Einkommenssituation wandeln kann. 
Als Zwischenfazit bleibt festzuhalten, dass die Einführung einer Versicherung Bildungsinvestitionen begünstigt, da Konsumglättung zwischen den beiden Gesundheitszuständen betrieben wird. Die tatsächliche Höhe der Prämie errechnet sich aus dem Durchschnitt der erwarteten Versicherungsausgaben, so dass ein erwarteter Anstieg der Ausgaben zur Behandlung der Krankheitsschäden $L$ bei gleicher Erkrankungswahrscheinlichkeit stets zu einem Anstieg der Prämie in der zweiten Periode führt. Somit stellt sich an dieser Stelle zusätzlich die Frage, inwiefern sich Veränderungen der Prämie auf die Bildung auswirken. ${ }^{134}$

Aus den Bedingungen erster Ordnung folgt für den Einfluss der Prämie unter Verwendung des Totalen Differentials:

$$
\frac{d b_{P}}{d P_{2}}=\frac{\pi\left[\alpha \beta_{P}^{\prime}-(1+r)\right] u_{2 G, P}^{\prime \prime}-(1-\pi)(1+r) u_{2 K, P}^{\prime \prime}}{\pi \alpha \beta_{P}^{\prime \prime} u_{2 G, P}^{\prime}+\pi\left[\alpha \beta_{P}^{\prime}-(1+r)\right] \alpha \beta_{P}^{\prime} w u_{2 G, P}^{\prime \prime}} .
$$

Der Nenner ist aufgrund der unterstellten Konkavität von Nutzen- und Bildungsproduktionsfunktion sowie aufgrund des in (7.28) beschriebenen Zusammenhangs zwischen Bildung und Zins definitionsgemäß negativ. Damit ergibt sich ein negativer Einfluss der Prämienerhöhung auf die Bildung für einen positiven Zähler. Umformen von Gleichung (7.27) und anschließendes Einsetzen in den Zähler von (7.29) ergibt folgende Bedingung für einen negativen Effekt auf die Bildungskapitalakkumulation:

$$
-\frac{u_{2 G, P}^{\prime \prime}}{u_{2 G, P}^{\prime}}<-\frac{u_{2 K, P}^{\prime \prime}}{u_{2 K, P}^{\prime}}
$$

Diese Bedingung ist für Nutzenfunktionen mit global abnehmender Risikoaversion gegeben. ${ }^{135}$ Damit ist der Einfluss einer Prämienerhöhung bei einer unterstellten Nutzenfunktion, deren Verlauf dem in (7.30) definierten entspricht, stets negativ. Bildungsinvestitionen werden gegen Ersparnisse substituiert. Die Begründung hierfür liegt in den Finanzierungsmöglichkeiten der Prämienerhöhung.

\footnotetext{
${ }^{134}$ Unerheblich ist in diesem Zusammenhang, ob sich tatsächlich Änderungen in der Prämienhöhe ergeben oder ob diese lediglich erwartet werden und es de facto nicht zu einer Änderung kommt, da die erwartete Prämienhöhe für die Bildungsentscheidung maßgeblich ist. Folglich ist nicht nur auf die tatsächliche Entwicklung, sondern auch auf die langfristigen Erwartungen der Individuen zu achten.

${ }^{135} \mathrm{Zu}$ einer detaillierten Erläuterung der Risikoaversionsmaße siehe Arrow (1976).
} 
So kann das aufgrund der erhöhten Belastung im gesunden Zustand zusätzlich benötigte Einkommen aus beiden Einkommensquellen gleichermaßen stammen, während die Bedeutung der Ersparnisse im Krankheitsfall zunimmt. Bei einem konstanten Konsumniveau in der ersten Periode müssen die Bildungsinvestitionen zum Zweck des finanziellen Ausgleichs dementsprechend sinken.

Während sich eine Prämienanhebung negativ auf die Akkumulation von Bildungskapital auswirkt, ist das System der Krankenversicherung gegenüber Änderungen im Bildungskapital und damit gegenüber Beschäftigungs- und Lohnschwankungen zunächst robust. Die Zahlung der Prämie ist vom Lohn unabhängig und kann daher auch aus den Ersparnissen geleistet werden. Negative Konsequenzen für das Krankenversicherungssystem ergeben sich erst dann, wenn der Grundlohn $w$ aufgrund der geringeren Qualität der angebotenen Arbeitskraft bei Betrachtung weiterer Perioden absinkt, da sich hieraus erneut Substitutionseffekte zugunsten der Arbeitszeit ergeben würden. ${ }^{136}$

Darüber hinaus zeigt sich, dass eine einkommensunabhängige Prämienfinanzierung der Krankenversicherung keine Verzerrungen mit sich bringt, da sämtliche Einkommensarten durch das System gleich behandelt werden. Eine Vermeidung von Prämienzahlungen, indem eine Einkommensquelle einer anderen vorgezogen wird, ist nicht möglich. Aus verteilungspolitischer Sicht werden hier geringe Einkommen benachteiligt, wenn die Prämie gemäß des Prinzips einer gruppenmäßigen Äquivalenz für alle Einkommensschichten in gleicher Höhe zu entrichten ist. Wird jedoch ein maximaler Eigenanteilssatz festgelegt, bei dessen Überschreiten Ansprüche auf staatliche Transfers bestehen, ist zumindest sichergestellt, dass die wirtschaftliche Leistungsfähigkeit des Einzelnen nicht überschritten wird. ${ }^{137}$

Bei der Finanzierung des Umverteilungsvolumens muss indes sichergestellt sein, dass sich hierdurch nicht erneut unerwünschte Verzerrungen ergeben, indem eine Lohnsteuer erneut die Rendite von Bildungsinvestitionen belastet. Darüber hinaus kann eine Begrenzung des Anspruchs auf finanzielle Unterstützung die Unterlassung von Bildungsinvestitionen zugunsten des Gegenwarts-

\footnotetext{
${ }^{136}$ Nur durch eine Ausweitung der Arbeitszeit können höhere Prämienzahlungen in der ersten Periode finanziert werden. Als Konsequenz hieraus müsste der Bildungsaufwand weiter sinken, woraus sich langfristig ein weiter sinkender Lohnsatz ergeben kann.

${ }^{137}$ Konkrete Reformmodelle, die einen Einkommensausgleich vorsehen, sind beispielsweise das Konzept pauschaler Gesundheitsprämien der „Rürup-Kommission“ (vgl. Bundesministerium für Gesundheit und Soziale Sicherung (2003)), das so genannte Rürup-Wille-Modell (vgl. Rürup und Wille (2004)) sowie die Bürgerpauschale (vgl. Sachverstăndigenrat zur Begutachtung der gesamtwirtschaftlichen Entwicklung (2003)). Zu weiteren Gesundheitsreformmodellen siehe z. B. Henke et al. (2003).
} 
konsums fördern. Liegt das erwartete Einkommen eines Individuums dicht unter der Zuschussberechtigungsgrenze und würden Bildungsmaßnahmen den Lohnsatz entsprechend anheben, geht die Zuschussberechtigung verloren, so dass nach Abzug aller Zahlungen letztlich weniger Einkommen für Konsum und Ersparnis zur Verfügung steht.

\subsection{Einführung einer steuerfinanzierten Krankenversicherung}

\subsubsection{Finanzierung über eine proportionale Lohnsteuer}

Als Alternative zum Prämiensystem besteht die Möglichkeit, die Krankenversicherung über lohnproportionale Beitragszahlungen oder eine entsprechende Lohnsteuer zu finanzieren, um dem Leistungsfähigkeitsprinzip stärker Rechnung zu tragen. Angelehnt an die Finanzierung eines Bismarckschen Krankenversicherungssystems soll an dieser Stelle der Einfluss einer steuerfinanzierten Krankenversicherung auf die Bildungsinvestitionen untersucht werden. ${ }^{138}$ Die Mitgliedschaft in einem solchen System ist verpflichtend, die Möglichkeit eines privaten Versicherungsschutzes besteht ausschließlich über Zusatzversicherungen und wird daher im Folgenden in die privaten Konsumausgaben integriert und somit nicht explizit berücksichtigt. ${ }^{139}$

Die Lohnsteuerzahlungen erfolgen über die proportionalen Steuertarife $t \in(0,1)$ in der ersten und $\tau \in(0,1)$ in der zweiten Periode. ${ }^{140}$ Diese Steuersätze gelten für alle Individuen gleichermaßen und sind dementsprechend an dem Finanzbedarf der Versicherung, nicht aber an dem individuellen Erkrankungsrisiko ausgerichtet. Vielmehr wird die Finanzierung der Krankenversicherung ausschließlich durch das Erwerbseinkommen determiniert. ${ }^{141}$

\footnotetext{
${ }^{138}$ Zum Zweck der besseren Vergleichbarkeit mit Ansätzen aus der aktuellen Literatur wird an dieser Stelle ein steuerfinanziertes System vorgestellt. Im Vergleich mit einem beitragsfinanzierten System ergeben sich hier keine Unterschiede in der Wirkung auf die Bildungskapitalakkumulation.

${ }^{139}$ Eine gesonderte Berücksichtigung wäre vonnöten, wenn bewusste Investitionen in die Gesundheit mit in die Analyse einbezogen würden.

${ }^{140}$ Die Unterscheidung der Steuersätze wird vorgenommen, um Erwartungen über steigende oder sinkende Steuern zwischen den Perioden abbilden zu können. Diese beeinflussen die Erträge der Bildungsinvestitionen und werden somit in das Kalkül mit einbezogen.

${ }^{141}$ Eine steuerliche Berücksichtigung von Zinseinkünften erfolgt in Abschnitt 7.4.3. Von der Implementierung einer Beitragsbemessungsgrenze in das Modell wird aus Vereinfachungsgründen jedoch abgesehen.
} 
Das Optimierungsproblem lautet

$$
\max _{S, b} u\left(c_{1}\right)+\pi(h) u\left(c_{2 G}\right)+[1-\pi(h)] u\left(c_{2 K}\right)
$$

unter den Nebenbedingungen

$$
\begin{aligned}
& c_{1}=(1-t)(1-b) w-S, \\
& c_{2 G}=(1+r) S+(1-\tau)[1+\varphi] w, \\
& c_{2 K}=(1+r) S+(1-\tau) q w-(1-v) L, \\
& \sum_{i}\left(1-\pi_{i}\right)\left[v L_{i}+(1-\tau) q w\right]=\sum_{i} \pi_{i} \tau\left[1+\varphi_{i}\right] w .
\end{aligned}
$$

Für jeden positiven Steuersatz $t$ ist das Nettoeinkommen in der ersten Periode c. p. geringer als unter Sicherheit bzw. in der Situation ohne Krankenversicherung. Über das Konsumniveau lässt sich im Vergleich zum Prämiensystem keine Aussage treffen, da sich dieses durch den unterschiedlichen Anreiz zum Vorsorgesparen unterscheiden kann. Während die Prämienzahlungen im vorangegangenen Modellabschnitt lohnunabhängig und damit pro Periode konstant sind, unterscheiden sich Brutto- und Nettoerwerbseinkommen in einem lohnsteuerfinanzierten Modell, wobei die Grenzbelastung aufgrund der proportionalen Steuertarife konstant ist.

Aus Gleichung (7.31) folgt für die erste Periode, dass die beiden Kapitalarten Humankapital und Realkapital im Hinblick auf die Besteuerung ungleich behandelt werden. Die Ersparnisbildung erfolgt aus dem bereits besteuerten Erwerbseinkommen, während die Investitionen in die Bildung in der ersten Periode steuerfrei sind.

Bedingung (7.32) beschreibt den Konsum bei Gesundheit in der zweiten Periode. Das verfügbare Einkommen setzt sich aus den verzinsten Ersparnissen und dem Nettoerwerbseinkommen zusammen. Die Besteuerung des Lohneinkommens führt dazu, dass Humankapital zur Finanzierung des Krankenversicherungssystems herangezogen wird, Realkapital jedoch steuerfrei bleibt. Hieraus können sich Substitutionseffekte zugunsten der Ersparnisbildung ergeben, um Steuerzahlungen $\mathrm{zu}$ vermeiden. 
Für den Konsum im Krankheitsfall (Gleichung (7.33)) ergibt sich ein Einkommen aus den steuerfreien Ersparnissen sowie aus den Lohnersatzleistungen, die sich zwar in der Höhe nicht von den Leistungen bei Prämienfinanzierung unterscheiden, zusätzlich aber der Besteuerung unterliegen. Im Krankheitsfall erhält das Individuum zudem erneut einen Ausgleich für seine Ausgaben für medizinische Leistungen in Höhe von $v L$. Hierfür gilt, dass diese Leistungen unabhängig von den bisher gezahlten Steuern gewährt werden, so dass dem oben genannten Vermeidungsziel keine Restriktionen entgegenstehen. Für das Einkommen bei Krankheit gilt weiterhin, dass dieses aufgrund der Unzulässigkeit von privater Verschuldung zumindest den Selbstbeteiligungsanteil am Gesundheitsschaden decken muss, so dass $(1+r) S+(1-\tau) q w \geq(1-v) L$ gilt.

Ein weiterer wesentlicher Unterschied zu einem prämienfinanzierten System ergibt sich aus der Nullgewinnbedingung der Krankenversicherung (Gleichung (7.34)). Auf der linken Seite steht erneut die Summe der erwarteten Ausgaben, die jetzt durch die Summe der erwarteten Einnahmen gedeckt sein muss. Während die Einnahmen in einem Prämiensystem aufgrund der Einkommensunabhängigkeit sicher sind, hängen diese bei einer Lohnsteuerfinanzierung von den Erkrankungswahrscheinlichkeiten und damit von der Erwerbsfähigkeit der Individuen ab. Das bedeutet, dass Schwankungen im Erkrankungsrisiko aller Individuen nicht mehr nur die Ausgabenseite betreffen, sondern sich auch negativ auf die Einnahmeseite des Systems auswirken. Das erwartete Steueraufkommen wird sowohl durch die Zahl der Arbeitskräfte in der zweiten Periode als auch durch deren Einkommen und damit durch die Arbeitsproduktivität bestimmt. Je mehr Individuen erwerbsfähig sind, desto höher ist die Bemessungsgrundlage und desto geringer kann der Steuersatz gewählt werden, damit sämtliche Ausgaben durch die Versicherung gedeckt sind. Ein geringerer Steuersatz würde wiederum die Attraktivität der Bildungsinvestitionen steigern, da der Nettolohn ansteigt. Wird tatsächlich mehr Zeit in die Bildung investiert, ergeben sich hieraus zudem Produktivitätssteigerungen, die sich wiederum in Lohnsteigerungen und Steuersenkungen niederschlagen. ${ }^{142}$

142 Im Rahmen der hier gewählten Darstellung wird in der Ausbildungsperiode keine Versicherungsleistung in Anspruch genommen. In einem vollständigen Modell überlappender Generationen wären in der Nullgewinnbedingung der Versicherung beide Generationen gleichermaßen zu berücksichtigen. Dieser Fall bleibt aus Vereinfachungsgründen in der formalen Analyse ausgeklammert. Es sei darauf hingewiesen, dass die Höhe des optimalen Steuersatzes negativ von dem Erstattungssatz abhängt. Der Einfluss der Bildung ist hingegen ambivalent, da diese zum einen in der Jugend die Steuerzahlungen senkt und somit einen höheren Steuersatz nach sich zieht, zum anderen jedoch auch zu einer gesteigerten Arbeitsproduktivität und damit zu höheren Löhnen führt, so dass sich hieraus Steuersenkungseffekte ergeben würden. 
Um einen Vergleich zwischen den einzelnen Alternativen zu ermöglichen, wird erneut die optimale Grenzproduktivität der Bildung bestimmt. Für ein Nutzenmaximum ergeben sich aus (7.8) folgende Bedingungen erster Ordnung, wobei der Index $T$ den Fall einer lohnorientierten Besteuerung kennzeichnet:

$$
\begin{array}{ll}
S: & u_{1, T}^{\prime}=(1+r)\left[\pi(h) u_{2 G, T}^{\prime}+(1-\pi(h)) u_{2 K, T}^{\prime}\right], \\
b: \quad(1-t) u_{1, T}^{\prime}=\pi(h)(1-\tau) \alpha(h) \beta_{T}^{\prime}(b) u_{2 G, T}^{\prime} .
\end{array}
$$

Bedingung (7.35) beschreibt erneut die Möglichkeit der Konsumglättung über die Ersparnisse, während gemäß Bedingung (7.36) für sämtliche Bildungsbemühungen gilt, dass auf gegenwärtiges Einkommen verzichtet werden muss, um ein höheres zukünftiges Einkommen zu erhalten. ${ }^{143}$ Umformen von (7.36) nach dem Grenznutzen der ersten Periode, einsetzen in (7.35) und auflösen nach der Grenzproduktivität $\beta_{T}^{\prime}$ ergibt

$$
\beta_{T}^{\prime}=\frac{(1+r)}{\alpha} \frac{1-t}{1-\tau}\left[1+\frac{(1-\pi(h)) u_{2 K, T}^{\prime}}{\pi(h) u_{2 G, T}^{\prime}}\right] \text {. }
$$

Durch die Einführung einer lohnsteuerfinanzierten Krankenversicherung bestimmt nun zusätzlich das Verhältnis der Nettoquoten ${ }^{144}$ den für Bildung geplanten Zeitaufwand. Bleibt der Steuersatz im Zeitablauf konstant, dann gilt $t=\tau$ und Gleichung (7.37) wird erneut zu (7.14), so dass sich die Bildungsinvestitionen ausschließlich in den unterschiedlichen Grenznutzen in beiden Zuständen der zweiten Periode unterscheiden. Ist dies der Fall, dann wird bei Krankenversicherungsschutz mehr Zeit in die Bildung investiert, wenn das Verhältnis der Grenznutzen kleiner ist als ohne Versicherungsschutz:

$$
\beta_{T}^{\prime}<\beta_{R}^{\prime} \Leftrightarrow \frac{u_{2 K, T}^{\prime}}{u_{2 G, T}^{\prime}}<\frac{u_{2 K, R}^{\prime}}{u_{2 G, R}^{\prime}} .
$$

\footnotetext{
${ }^{143}$ Ein derartiger Zusammenhang wird auch in Blinder und Weiss (1976), S. 450, beschrieben.

${ }^{144}$ Die Nettoquote beschreibt hier das Verhältnis eines Nettowertes zu seiner jeweiligen Bruttogröße und ist damit immer genau das Gegenteil des Steuersatzes.
} 
Die Grenznutzenverhältnisse unterscheiden sich zum einen aufgrund der Steuerzahlungen, zum anderen aufgrund der Leistungen durch die Krankenversicherung im kranken Zustand. Die Versicherung lohnt sich dann, wenn der Konsum bei Krankheit den Konsum im Risikofall übersteigt. Hierfür müssen die verzinsten Ersparnisse zuzüglich der Versicherungsleistungen folglich größer sein als die verzinsten Ersparnisse ohne Versicherung, so dass $u_{2 K, T}^{\prime}<u_{2 K, R}^{\prime}$ gilt. Die Grenznutzen im Krankheitsfall sind in ihrer Höhe gleich, wenn sich der Konsum bei Krankheit hingegen nicht verändert, die verzinsten Ersparnisse also um den Deckungsanteil der Versicherung sowie die Lohnersatzleistungen sinken (d. h. $-\Delta S=(v L+(1-\tau) q w) /(1+r))$.

Ein Absinken der Ersparnisse kann sich zum einen dadurch ergeben, dass der Konsum in der ersten Periode bei konstantem Einkommen ausgeweitet wird. Bei unveränderter Konsumsumme sinken die Ersparnisse nur dann, wenn auch das Erwerbseinkommen zurückgeht, was wiederum auf eine Senkung der Arbeitszeit und damit auf eine erhöhte Bildungsaktivität zurückzuführen wäre. Bedingung (7.38) ist nun allerdings nur dann gegeben, wenn der Konsum ohne Krankenversicherung im Gesundheitsfall oberhalb des Konsumniveaus mit Krankenversicherungsschutz liegt, d. h. wenn $u_{2 G, T}^{\prime}>u_{2 G, R}^{\prime}$ ist. Die Zuwächse im Nettoeinkommen, die sich aus dem zusätzlichen Bildungskapital ergeben, müssen somit geringer ausfallen als der Ersparnisrückgang. Folglich müssten die jeweiligen Steuersätze bei gleichzeitig geringer Versicherungsleistung entsprechend hoch sein, damit dieser Fall Bestand hätte.

Im Folgenden wird davon ausgegangen, dass sich das Konsumniveau bei Krankheit durch die Einführung der Versicherung im Vergleich zum Konsum bei Unsicherheit erhöht. Je höher zudem die Varianz zwischen beiden Einkommenssituationen bei Krankheit ist, je höher also der Deckungsanteil der Versicherung sowie die Einkommensleistungen sind, desto stärker kann auf Einkommen im gesunden Zustand verzichtet werden, ohne dass Bedingung (7.38) verletzt wird.

Unterscheiden sich die Steuersätze der einzelnen Perioden, folgt aus (7.37) durch Auflösen nach $u_{2 K, T}^{\prime} / u_{2 G, T}^{\prime}$ als Bedingung für die erwartete Grenzproduktivität im Optimum:

$$
\pi \alpha \beta_{T}^{\prime}>\frac{1-t}{1-\tau}(1+r)
$$

Im Gegensatz zu den vorangegangenen Modellansätzen wird die erwartete optimale Grenzproduktivität nicht mehr nur durch die Verzinsung, sondern auch durch das Verhältnis der Nettoquoten bestimmt, sobald sich im Zeitablauf Ver- 
änderungen in der Besteuerung ergeben. Werden in der zweiten Periode Ausgabensteigerungen sowie ein Absinken der Beschäftigtenzahlen erwartet und bleiben die Versicherungsparameter konstant, muss im Gegenzug der Steuersatz angehoben werden, so dass $\tau>t$ gilt. In diesem Fall ist das Verhältnis der Nettoquoten größer Eins, so dass c. p. weniger Zeit in die Bildung investiert wird. Grund für die sinkende Nachfrage nach Bildung ist ein Absinken der erwarteten Bildungserträge im Verhältnis zum Nettoeinkommen der ersten Periode, so dass es lohnender wird, auf eine umfassende Ausbildung zu verzichten und einen Teil des Konsums über die Ersparnisse in die zweite Periode zu transferieren. In diesem Fall kann eine Krankenversicherung aufgrund des Anreizes zur Steuervermeidung dazu führen, dass weniger Zeit in die Bildung investiert wird, obwohl die Unsicherheit gemindert wird.

Verbessert sich hingegen die Einnahmenseite der Krankenversicherung aufgrund von Lohnzuwächsen oder aufgrund von steigenden Beschäftigtenzahlen, kann der Steuersatz in der zweiten Periode $\tau$ den Satz der ersten Periode $t$ unterschreiten. In diesem Fall wird die rechte Seite von Bedingung (7.39) kleiner, woraus sich bei einer konstanten Erkrankungswahrscheinlichkeit $\pi$ sowie bei gegebener Lerneffizienz $\alpha$ eine geringere erwartete Grenzproduktivität ergibt. In diesem Fall kann somit vermehrt Zeit für Bildungsinvestitionen aufgewendet werden, da das Nettoerwerbseinkommen im gesunden Zustand steigt, während die Leistungen der Versicherung unverändert bleiben. Es entsteht ein erhöhter Anreiz zur Bildungskapitalakkumulation. Die Begründung für diese Zusammenhänge liegt im Zeitpunkt der Besteuerung der beiden Kapitalarten. Während das Realkapital, das auf den Ersparnissen aus Periode 1 beruht, über die Lohnsteuer in der ersten Periode einer vorgelagerten Besteuerung unterliegt, ist die Akkumulation von Bildungskapital in der ersten Periode steuerfrei. Stattdessen werden sämtliche Erträge der Bildungsinvestitionen bei Gesundheit in der zweiten Periode nachgelagert besteuert, während die Zinserträge in der zweiten Periode steuerfrei sind. Ist der Steuersatz der ersten Periode größer als in der zweiten Periode, dann wird das Realkapital folglich stärker besteuert als das Bildungskapital, es kommt zu Substitutionseffekten zugunsten der Bildungsinvestitionen.

Die Entscheidung über Bildung und Ersparnis lässt sich wie folgt charakterisieren: Die Besteuerung des Erwerbseinkommens in der ersten Periode bestimmt den finanziellen Rahmen, in dem Konsum der ersten Periode sowie die Akkumulation der beiden Kapitalarten möglich sind. Die Erträge des Realkapitals in Form von Zinsen sind in der zweiten Periode steuerfrei und werden lediglich durch den Zinssatz determiniert. Die Erträge des Bildungskapitals hingegen werden zum einen durch den Steuersatz der zweiten Periode bestimmt, zum anderen durch die Produktivität des Individuums, mit der Zeitaufwand in Bildungskapital umgewandelt werden kann. Ein Vergleich zwischen (7.39) und 
(7.16) lässt damit keinen eindeutigen Schluss darüber zu, ob eine steuerfinanzierte Krankenversicherung die Bildungsinvestitionen positiv beeinflusst. Zum einen ermöglicht die Einführung einer Krankenversicherung ein höheres Einkommen im kranken Zustand der zweiten Periode, indem ein Teil des finanziellen Schadens sowie des Lohnausfalls übernommen wird, zum anderen senkt diese jedoch die Erträge aus den Bildungsinvestitionen, woraus eine vermehrte Ersparnisbildung resultieren kann.

\subsubsection{Vergleich der Versicherungssysteme}

Die in Kapitel 7.4.1 genannten Zusammenhänge lassen sich analog zu den vorangegangenen Modellansätzen grafisch darstellen. Verglichen mit dem Prämienmodell bleibt die Beziehung zwischen der Sparquote und den Kapitaleinkünften unverändert, da lediglich das Human-, nicht aber das Realkapital in der zweiten Periode besteuert wird. Der zweite Quadrant in Abbildung 18 ist folglich mit dem in Abbildung 17 identisch. Für die übrigen drei Quadranten ergeben sich hingegen Veränderungen in den Kurvenverläufen aus der Systemumstellung.

Um Vergleiche mit der Risikosituation sowie einer prämienfinanzierten Krankenversicherung anstellen zu können, wird im ersten Quadranten erneut der Trade-off zwischen Bildungs- und Sparquote abgebildet und nicht der Zusammenhang zwischen der Bildungszeit und den absoluten Ersparnissen. Eine Ausweitung der Bildungszeit führt bei einem in der ersten Periode konstantem Konsum zu einem Rückgang der Ersparnisse um den Nettolohnsatz, da sich das Erwerbseinkommen um genau diesen Betrag verringert. Im Vergleich hierzu gehen die Ersparnisse in der Situation ohne Versicherung im Umfang des gesamten entgangenen Bruttolohnes zurück. Unter Verwendung der Sparquote beträgt die Grenzrate der Substitution erneut $\mathrm{d} s / \mathrm{d} b=-(1-s) /(1-b)$. Somit verschiebt sich die Konsummöglichkeitenkurve im Vergleich zur Risikosituation ohne Versicherung (Abbildung 16) bei gleich bleibendem Konsumniveau Richtung Ursprung.

Von besonderem Interesse sind darüber hinaus die Veränderungen, die sich hieraus im Vergleich mit dem Prämiensystem ergeben, da die Konsummöglichkeitenkurve jeweils die maximale Bildungszeit sowie die maximal mögliche Sparquote festlegt. Aus den Grenzraten der Substitution ${ }^{145}$ folgt zunächst, dass die Konsummöglichkeitenkurve bei Prämienfinanzierung steiler verläuft. Hieraus ist jedoch noch nicht ersichtlich, in welchem System mehr Zeit in die Bil-

${ }^{145} \mathrm{Im}$ Prämienmodell gilt $\frac{d s}{d b}=-\frac{(1-s) w}{(1-b) w-P_{1}}$. 
dung investiert werden kann und wie sich die Finanzierung auf die Ersparnisbildung auswirkt.

Für die Versicherung gilt, dass die Leistungsausgaben $(v L+q w)$ jeweils unabhängig von der Finanzierungsart sind, so dass sich die Einnahmen im Durchschnitt entsprechen müssen. Läge nun die Konsummöglichkeitenkurve bei Prämienfinanzierung global unterhalb derjenigen im Steuersystem, würde dies bedeuten, dass die Prämie stets oberhalb der zu zahlenden Steuer läge, so dass die durchschnittliche finanzielle Gleichbehandlung nicht mehr gegeben ist. Im Gegensatz hierzu würden die Prämieneinnahmen stets unterhalb der Steuereinnahmen liegen, wenn die Bildungsquote bei Prämienfinanzierung mindestens derjenigen im Steuerfall entspricht. Da dies ebenfalls nicht zulässig ist, lässt sich hieraus schließen, dass die maximale Bildungsquote im Prämienfall unterhalb derjenigen bei Steuerfinanzierung liegen muss.

Im Hinblick auf Unterschiede in der maximalen Sparquote lassen sich ebenfalls detaillierte Aussagen treffen. Im Fall identischer maximaler Sparquoten müsste der Steuersatz genau dem Prämienanteil am Gesamtlohn entsprechen $(t=P / \mathrm{w})$. Für jede positive Bildungszeit würde die Prämie dann oberhalb der zu zahlenden Steuer liegen, da diese negativ von den Bildungsinvestitionen abhängt. $\mathrm{Da}$ diese Randlösung jedoch bildungspolitisch nicht von Interesse ist, folgt hieraus, dass ein Steuersystem verglichen mit pauschalen Prämien mehr Bildung zulässt, im Gegenzug aber die Sparquote senkt. Der Schnittpunkt beider Konsummöglichkeitenkurven ergibt sich dort, wo die durchschnittlichen Steuerzahlungen genau der Prämie entsprechen. Links von diesem Schnittpunkt ist bei gleicher Bildungsquote im Steuermodell weniger Ersparnis möglich, da die Steuerzahlungen die Prämienhöhe übersteigen.

Im III. Quadranten ergeben sich durch die Lohnbezogenheit der Steuerzahlungen ebenfalls Unterschiede. Aufgrund der Annahme, dass Ersparnisse nicht besteuert werden, ist die Steigung der Konsumgeraden jeweils gleich in Höhe von Eins, d. h. zusätzliche Ersparnisse erhöhen den Konsum bei Krankheit jeweils in vollem Umfang. Im Hinblick auf die Steigung sind die Konsumgeraden damit gleich, nicht aber hinsichtlich ihrer Lage. Der Ordinatenabschnitt kennzeichnet den Konsum bei Krankheit, wenn aufgrund der Lohnfortzahlungen vollständig auf Ersparnisbildung verzichtet wird. Die Lage der Konsumgeraden hängt nun maßgeblich von der Höhe der Prämie bzw. der Steuerzahlungen ab. Zunächst wird angenommen, dass die Prämie in der zweiten Periode genau den Steuerzahlungen aus der Lohnfortzahlung entspricht, so dass $P_{2}=\tau q w$ gelten muss. Da die Prämie in beiden Zuständen in identischer Höhe erhoben wird, müsste in diesem Fall ebenso $P_{2}=\tau[1+\varphi] w$ gelten, was für alle $q<1$ nicht möglich ist. 
Abbildung 18: Bildungsinvestitionen bei lohnsteuerfinanziertem Krankenversicherungsschutz

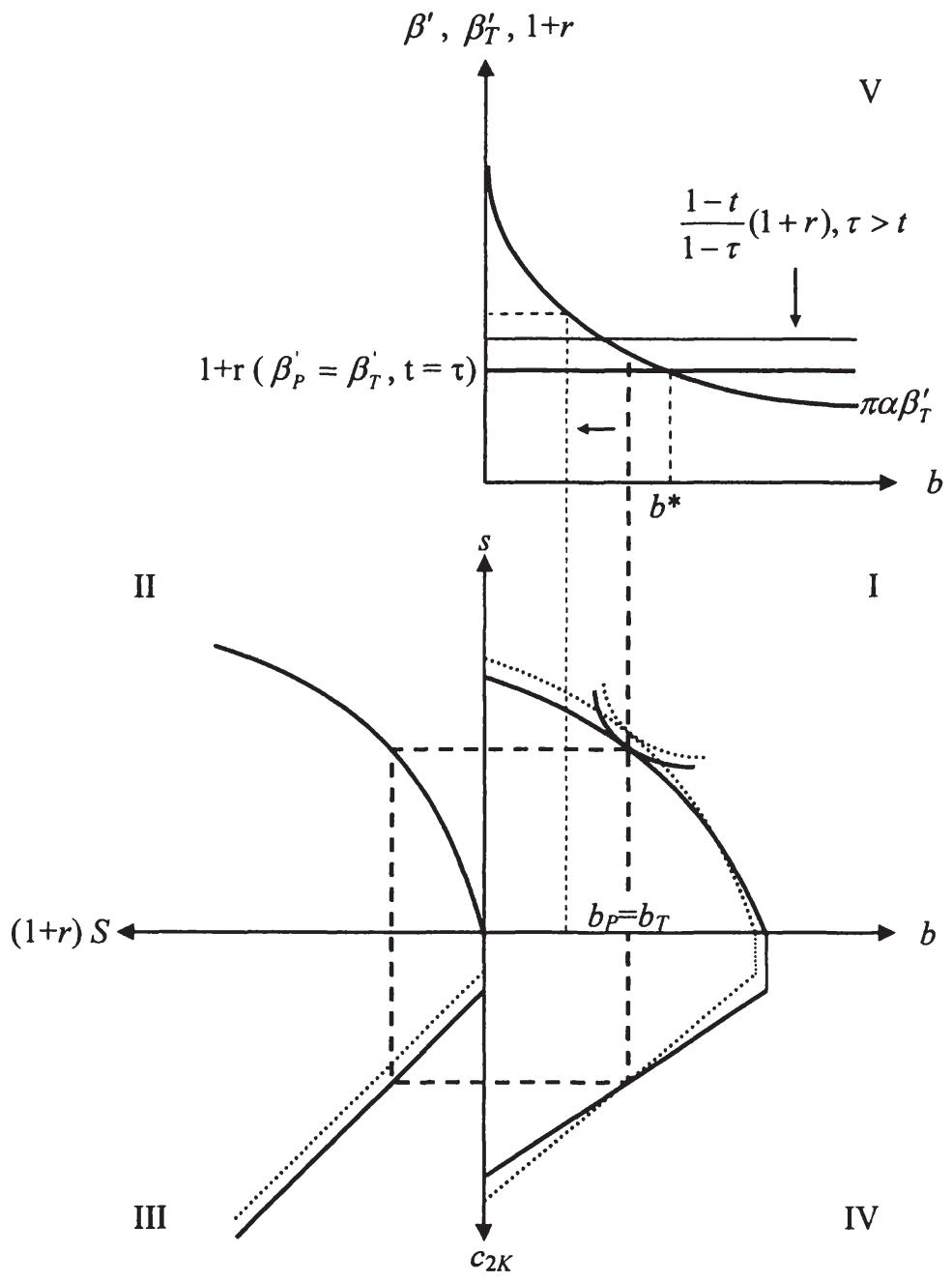

Prämienfinanzierung

— Steuerfinanzierung

Quelle: Eigene Darstellung. 
Aus diesem Grund muss die Prämie in ihrer Höhe den mit den Eintrittswahrscheinlichkeiten gewichteten Steuereinnahmen in beiden Zuständen entsprechen, so dass sich die Prämie anhand von $P_{2}=\tau[(1-\pi) q w+\pi(1+\varphi) w]$ errechnet. Damit liegt die Prämie stets oberhalb der Steuerlast im Krankheitsfall.

Es zeigt sich, dass die finanzielle Belastung im Steuersystem bei Gesundheit größer als bei Krankheit ist, da das Erwerbseinkommen definitionsgemäß über dem Einkommen bei Krankheit liegt. Im Prämienmodell ist die Belastung hingegen in beiden Zuständen gleich. Folglich sinkt auch die Bedeutung der Ersparnisse im Steuermodell, so dass die Konsumkurve oberhalb des Konsums bei Prämienfinanzierung liegt. ${ }^{146}$

Für den Einfluss der Bildungsquote auf den Konsum bei Krankheit im IV. Quadranten folgt analog zum Prämiensystem, dass nunmehr das volle Bildungspotential ausgeschöpft werden kann, da mit Krankheit keine finanzielle Restriktion mehr verbunden ist. Aufgrund der Besteuerung des Erwerbseinkommens wirkt sich eine Ausweitung der Bildungszeit jedoch nicht mehr so stark auf das verfügbare Einkommen aus wie in den Situationen ohne Versicherung oder bei Prämienfinanzierung. Entsprechend verläuft die Konsumgerade bei Steuerfinanzierung im IV. Quadranten mit geringerer Steigung. ${ }^{147}$

Im Vergleich zu dem Prämiensystem ist der Konsum bei Krankheit ohne Zeitaufwand für Bildungszwecke im Steuersystem geringer, bei hohem Bildungsaufwand kann hingegen mehr konsumiert werden. Dieser Zusammenhang lässt sich über die Bedeutung der Ersparnisse im jeweiligen Versicherungsfall begründen. In Bezug auf die Prämie gilt, dass die Summe aus Lohnersatzleistungen und Ersparnissen ausreichen muss, um die Prämienzahlungen auch bei Krankheit leisten zu können. Die Steuerzahlungen sind hingegen direkt an die Lohnersatzleistungen gekoppelt, so dass hier unabhängig von der Höhe der Ersatzleistungen keine Mindestersparnissumme erforderlich ist. Somit ist der maximal mögliche Bildungsaufwand im Steuermodell größer als im Prämienmodell. Gleichzeitig gilt für den maximal möglichen Konsum ohne Bildung, dass dieser aufgrund der höheren maximalen Sparquote im Prämienmodell größer ist.

\footnotetext{
146 Wie weit die Konsummöglichkeiten im Krankheitsfall voneinander entfernt liegen, hängt maßgeblich von den Bildungsbemühungen aller Versicherten und damit von den Einkommenssteigerungen $\mathrm{ab}$. Während die Prämienbelastung nur durch eine Veränderung der Ausgabenstruktur oder durch weniger Krankheitsfälle gesenkt werden kann, können Steuersenkungen selbst bei einer gleich bleibenden Erkrankungswahrscheinlichkeit realisiert werden, wenn die Bildungsanstrengungen der übrigen Individuen mit entsprechenden Produktivitătszuwächsen einhergehen.

${ }^{147}$ Die Steigung der Geraden verändert sich von - $(1+r) w$ auf $-(1+r)(1-t) w$.
} 
Der Schnittpunkt der Konsummöglichkeitenkurven im I. Quadranten kennzeichnet die Situation, in der sich die finanziellen Belastungen durch die Versicherung entsprechen. Es kann darüber hinaus gezeigt werden, dass sich die Konsumkurven bei Krankheit bereits bei einem geringeren Bildungsniveau schneiden. Nach wie vor ist das Konsumniveau in der ersten Periode für beide Systeme identisch und fest vorgegeben. Die Konsum- und Bildungsniveaus im IV. Quadranten stimmen nun genau dann überein, wenn die nachfolgende $\mathrm{Be}-$ dingung

$$
t(1-b) w+\frac{1}{1+r}(\tau q w)=P_{1}+\frac{1}{1+r} P_{2}
$$

die intertemporale Belastung durch die Krankenversicherung widerspiegelt. Es ist weiterhin bekannt, dass die Steuerzahlungen bei Krankheit durch die Umverteilung zwischen den Zuständen unterhalb der Prämie in der zweiten Periode liegen, d. h. $\tau q w<P_{2}$. Für Gleichung (7.40) folgt hieraus, dass die Steuerzahlungen in der ersten Periode die Prämie übersteigen müssen. Dies ist in allen Bereichen links des Schnittpunktes im I. Quadranten der Fall.

Aus den genannten Zusammenhängen ergibt sich folgende Situation im V. Quadranten: Wird zunächst angenommen, dass sich die Steuersätze der beiden Perioden entsprechen, ist nach wie vor die Verzinsung die entscheidende Bezugsgröße für die optimale Grenzproduktivität der Bildung, der gewählte Zeitaufwand kann demjenigen im Prämiensystem entsprechen $\left(b_{T}=b_{P}\right)$.

Sind die Steuersätze in beiden Perioden jedoch unterschiedlich, kann sich hieraus eine Verzerrung für die Bildungsentscheidung ergeben. Liegt der Steuersatz der ersten Periode oberhalb des Steuersatzes in der zweiten Periode, verschiebt sich die Zinsgerade in Abbildung 18 nach unten. Der Schnittpunkt mit dem erwarteten Grenzertrag der Bildung verschiebt sich entsprechend nach rechts, so dass die Bildungszeit ausgeweitet werden kann, ein Verharren im alten Optimalpunkt $b_{T}$ ist jedoch ebenso weiterhin möglich. ${ }^{148}$ Steigt der Steuersatz hingegen über die Perioden an, so dass $\tau>t$ ist, dann führt diese Entwicklung zu einer Parallelverschiebung der Zinsgeraden nach oben. Der neue Schnittpunkt im V. Quadranten befindet sich nun links vom alten Optimum, weshalb der Bildungsaufwand nicht weiter aufrechterhalten werden kann (siehe Bedingung (7.39)). Die in Bildung investierte Zeit wird verkürzt. In diesem Fall führt ein

\footnotetext{
${ }^{148}$ Dieser Zusammenhang ist auf die Ungleichung in (7.39) zurückzufuhren. Welcher Zeitaufwand für Bildung tatsächlich gewählt wird, hängt von den Präferenzen und der Risikobereitschaft der Individuen ab. Hierüber wurden im betrachteten Modell keine weiteren Annahmen getroffen.
} 
Steuersystem dazu, dass weniger Zeit in die Bildung investiert wird, obwohl die Rahmenbedingungen so gesetzt sind, dass insgesamt mehr Bildungszeit möglich ist.

\section{Veränderungen des Gesundheitszustandes $h$}

Analog zu den vorangegangenen Kapiteln stellt sich die Frage, welchen Einfluss eine Verbesserung der Gesundheit auf die Bildungsentscheidung ausübt, wenn ein steuerfinanzierter Krankenversicherungsschutz vorliegt und dementsprechend nur noch ein Teil der anfallenden finanziellen Aufwendungen privat zu finanzieren ist. Aus (7.36) folgt

$$
\begin{aligned}
\frac{d b_{T}}{d h} & =-\frac{1-\tau}{1-t} \frac{\beta_{T}^{\prime}\left[\pi^{\prime} \alpha+\pi \alpha^{\prime}\right]}{\Psi_{T}} u_{2 G, T}^{\prime}-\frac{\pi^{\prime}(1+r)}{\Psi_{T}} U_{T}^{\prime} \\
& -(1-\tau) \frac{\alpha^{\prime} \beta_{T} \pi\left[\frac{1-\tau}{1-t} \alpha \beta^{\prime}-(1+r)\right] w}{\Psi_{T}} u_{2 G, T}^{\prime \prime}
\end{aligned}
$$

mit

$$
\begin{aligned}
U_{T}^{\prime} & =u_{2 K, T}^{\prime}-u_{2 G, T}^{\prime} \\
\Psi_{T} & \equiv \pi \frac{1-\tau}{1-t} \alpha \beta_{T}^{\prime \prime} u_{2 G, T}^{\prime} \\
& +\pi\left[\frac{1-\tau}{1-t} \alpha \beta_{T}^{\prime}-(1+r)\right](1-\tau) \alpha \beta_{T}^{\prime} w u_{2 G, T}^{\prime \prime}<0 .
\end{aligned}
$$

Es zeigt sich, dass sich in der Richtung der einzelnen Partialeffekte keine Veränderungen gegenüber einer Prämienfinanzierung ergeben. Während die Prämienzahlungen jedoch ausschließlich über die Nutzenfunktion in das Kalkül einfließen, spielen die einzelnen Steuersätze hier explizit eine Rolle. Eine erwartete Steuersenkung in der zweiten Periode würde beispielsweise auf der einen Seite dazu führen, dass die Erträge aus den Bildungsinvestitionen ansteigen und somit der Anreiz, in die Bildung zu investieren, zunimmt. Auf der anderen Seite kann Bildung dann leichter gegen Ersparnisse substituiert werden, ohne dass auf Einkommen bei Gesundheit verzichtet werden muss. 


\section{Veränderungen des Steuersatzes $\tau$}

Waren die Vergleiche zwischen den einzelnen Unsicherheitssituationen bislang nur auf die optimale Bildungsproduktivität ausgerichtet, so ist weiterhin von Interesse, wie sich Steuererhöhungen direkt auf die Bildungsinvestitionen auswirken. Ein niedriger Steuersatz in der ersten Periode ermöglicht bei konstantem Konsum sowie gleich bleibenden Ersparnissen eine vergleichsweise hohe Bildungsquote. Wäre folglich vor Antritt einer Ausbildung bekannt, dass der Steuersatz $t$ gesenkt wird, so würde dies zu positiven Bildungseffekten führen. Im Rahmen des vorliegenden Modells werden derartige Steuersenkungen jedoch nicht näher betrachtet, da der Steuersatz der ersten Periode fest vorgegeben ist und somit ein unveränderliches Datum darstellt.

Anders verhält es sich mit dem zukünftigen Steuersatz $\tau$. Dieser kann ex ante nicht mit Sicherheit festgestellt werden, da er zum einen vom Finanzbedarf, zum anderen aber auch vom Bildungsverhalten der übrigen Individuen abhängt. Somit ist es möglich, dass sich die Erwartungen über die Entwicklung der Besteuerung in der zweiten Periode zwischen einzelnen Individuen unterscheiden. Nun wird hierüber auch die Bereitschaft, in die Bildung zu investieren, determiniert, indem sich der Nettoertrag der Bildungsinvestitionen aus den Steuerzahlungen ableitet.

Das Totale Differential aus (7.36) ergibt für den Einfluss des Steuersatzes der zweiten Periode auf die Bildungsinvestitionen folgendes Resultat:

$$
\begin{aligned}
\frac{d b_{T}}{d \tau} & =\frac{1}{(1-t)} \frac{\pi \alpha \beta_{T}^{\prime}}{\Psi_{T}} u_{2 G, T}^{\prime} \\
& -\frac{(1-\pi)(1+r) q w}{\Psi_{T}} u_{2 K, T}^{\prime \prime}+\frac{\Omega_{T}[1+\varphi]}{\Psi_{T}} u_{2 G, T}^{\prime \prime}
\end{aligned}
$$

mit

$$
\Omega_{T} \equiv \pi\left[\frac{1-\tau}{1-t} \alpha \beta^{\prime}-(1+r)\right] w>0 .
$$

$\Psi_{T}$ wird erneut durch Bedingung (7.43) definiert. Der erste Term im Zähler von Gleichung (7.44) beschreibt einen negativen Einfluss eines höheren Steuersatzes auf die Bildungsinvestitionen, indem die erwarteten zusätzlichen Erträge einer 
weiteren Bildungseinheit sinken. Hieraus folgt ein positiver Anreiz für zusätzliche Ersparnisbildung.

Der zweite Term in Gleichung (7.44) bestimmt die Höhe des Substitutionseffekts, der mit der Steuererhöhung einhergeht und ebenfalls zu einem Rückgang der Bildungsinvestitionen führt. Durch die Steuererhöhung wird der Nettobetrag der Lohnersatzleistungen gemindert, so dass mehr Ersparnisse gebildet werden müssen, um das Konsumniveau im Krankheitsfall konstant zu halten. Positiv wirkt sich hingegen der Einkommenseffekt (3. Term) auf die Bildungsinvestitionen aus, welcher eine gezielte Erhöhung des Bruttoeinkommens aufgrund der Minderung des Nettoeinkommens bei Gesundheit beschreibt. Welcher dieser genannten Effekte überwiegt, hängt erneut von der Risikobereitschaft des betreffenden Individuums sowie von den jeweiligen Erwartungseinkommen ab, so dass die Bedeutung eines steigenden Steuersatzes für die Bildungsinvestitionen nicht eindeutig ist. ${ }^{\text {199 }}$

\subsubsection{Erweiterung der Bemessungsgrundlage um Zinseinkünfte}

In dem vorangegangenen Modellansatz wurde in Anlehnung an die beitragsfinanzierte GKV nur das Erwerbseinkommen mit einer Steuer belegt, um die Krankenversicherung zu finanzieren. Damit wurden Ersparnisse relativ begünstigt, so dass es zu Substitutionseffekten zugunsten der Ersparnisbildung kommen konnte, sobald mit einer Steuererhöhung in der zweiten Periode gerechnet wurde. In diesem Abschnitt wird die Bemessungsgrundlage für die Besteuerung um die Zinseinkünfte in der zweiten Periode erweitert. Dieses Vorgehen hat zur Konsequenz, dass die Krankenversicherung nicht nur über das Einkommen im gesunden Zustand der zweiten Periode sowie über die Lohnersatzleistungen finanziert wird, sondern auch über die gesamten Zinseinnahmen, da diese zwischen den Zuständen identisch sind.

Der Konsum in der ersten Periode wird erneut durch (7.31) bestimmt. Die Konsumbedingungen für die beiden Zustände der zweiten Periode verändern sich zu

\footnotetext{
149 Snow und Warren (1990) zeigen, dass die Reaktion der Humankapitalinvestitionen auf die Einkommensentwicklung davon abhăngig ist, ob es sich bei Humankapital um ein normales oder um ein inferiores Gut handelt. Grundlage hierfür ist eine mit steigendem Einkommen abnehmende Risikoaversion. Je mehr Zeit nun in Bildung investiert wird, desto großer ist die Varianz zwischen dem maximal erreichbaren Einkommen und den Einkommensverlusten, die sich bei Erwerbsunfähigkeit ergeben, und desto größer ist auch die Unsicherheit über den zukünftigen Lohnsatz. Ist Humankapital ein inferiores Gut, dann fürt ein Anstieg im Risiko der Investitionen dazu, dass die Bereitschaft, dieses Risiko zu übernehmen, sinkt, so dass weniger investiert wird. Ist Humankapital jedoch ein normales Gut, dann steigen die Investitionen mit zunehmendem Risiko.
} 


$$
c_{2 G}=S+(1-\tau)(r S+[1+\varphi] w)
$$

und

$$
c_{2 K}=S+(1-\tau)[r S+q w]-(1-v) L
$$

Im Vergleich mit einer rein lohnbezogenen Besteuerung führt die zusätzliche Besteuerung der Zinseinkünfte c. p. zu einem niedrigeren Konsumniveau in beiden Zuständen, wenn die Zeitallokation sowie die Sparquote unverändert bleiben. Die Nullgewinnbedingung der Versicherung verändert sich zu

$$
\sum_{i}\left(1-\pi_{i}\right)\left[v L_{i}+(1-\tau) q w\right]=\sum_{i} \tau\left[\pi_{i}\left(1+\varphi_{i}\right) w+r S_{i}\right]
$$

Im Unterschied zu der Versicherungsbedingung (7.34) bestimmen sich die Einnahmen der Versicherung nicht mehr nur aus dem erwarteten Erwerbseinkommen, sondern auch aus den sicheren Zinserträgen. Für die Finanzierung der Krankenversicherung folgt zum einen, dass die Finanzierung in geringerem $\mathrm{Maße}$ von der Zahl der Erwerbstätigen abhängig und damit robuster gegenüber Beschäftigungsschwankungen ist. Zum anderen wird das Erwerbseinkommen entlastet, da sämtliche Einkommensarten zur Finanzierung der Versicherung herangezogen werden.

Analog zu den vorangegangenen Überlegungen ist der Deckungssatz $v$ nicht frei wählbar, da dieser von den Steuerzahlungen abhängt. Damit sind wiederum $b$ und $s$ die einzigen Entscheidungsvariablen. Die Bedingungen erster Ordnung lauten $^{150}$

$$
b:(1-t) u_{1, S}^{\prime}=\pi(h)(1-\tau) \alpha(h) \beta_{S}^{\prime}(b) u_{2 G, S}^{\prime}
$$

analog zu Gleichung (7.36), und

$$
S: \quad u_{1, S}^{\prime}=[1+(1-\tau) r]\left[\pi(h) u_{2 G, S}^{\prime}+(1-\pi(h)) u_{2 K, S}^{\prime}\right]
$$

${ }^{150}$ Der Index $S$ bezeichnet eine zusätzliche Besteuerung der Zinseinkünfte. 
Für die erwartete Grenzproduktivität im Optimum ergibt sich hieraus durch Gleichsetzen und Umformen

$$
\pi \alpha \beta_{S}^{\prime}>(1-t)\left(\frac{1}{1-\tau}+r\right) .
$$

Der Vergleich mit der Situation bei reiner Erwerbseinkommensbesteuerung zeigt, dass sich eine gleichmäßige Besteuerung der beiden Einkunftsarten Zinssowie Erwerbseinkommen weniger verzerrend auf die Bildungsentscheidung auswirkt, da Realkapital- und Humankapitaleinkommen nicht mehr unterschiedlich behandelt werden. Die rechte Seite in Bedingung (7.51) ist kleiner als die rechte Seite von (7.39), so dass sich hieraus eine geringere erwartete Grenzproduktivität der Bildung und damit einhergehend ein erhöhter Zeitaufwand für die Bildungskapitalakkumulation ergeben können. Dieser Zusammenhang impliziert wiederum, dass bei gleichmäßiger Besteuerung ein höherer Anreiz besteht, Zeit in die Bildung zu investieren, bis die erwartete Nettogrenzproduktivität der Bildung, $\pi \alpha \beta_{S}^{\prime}$, nur noch marginal über der Nettoverzinsung des Kapitaleinkommens liegt. Folglich erhöht die Ausweitung der Bemessungsgrundlage den Reiz weiterer Investitionen in die Bildung, da Anlagen in Finanzkapital in ihrer Attraktivität sinken.

\section{Veränderungen des Gesundheitszustandes $h$}

Es scheint, als wäre ein Modell, das beide Kapitaleinkünfte gleichermaßen besteuert, einem rein lohnsteuerfinanzierten Modell zumindest aus bildungspolitischen Gesichtspunkten vorzuziehen, da das Erstgenannte einen höheren Bildungskapitalstock ermöglicht. Bedingung (7.51) lässt jedoch keine Aussagen darüber $\mathrm{zu}$, wie sich die Bildungsinvestitionen bei Parameteränderungen verhalten. Hier stellt sich erneut die Frage, wie sich eine exogene Veränderung der Gesundheit auf die Bildungsbereitschaft auswirkt (siehe hierzu die Gleichungen A1-A3 im Anhang dieses Kapitels). Trotz der geänderten Bemessungsgrundlage bleiben die einzelnen Partialeffekte in ihrer Richtung erhalten, auch wenn sich diese in ihrer Ausprägung verändern.

Im Fall einer reinen Lohnsteuerfinanzierung führt ein Absinken der Erkrankungswahrscheinlichkeit dazu, dass die Ersparnisbildung an Bedeutung verliert, da die Erzielung eines Erwerbseinkommens immer wahrscheinlicher wird. Durch die zusätzliche Besteuerung der Zinseinkünfte wird dieser für die Bildung positive Effekt abgeschwächt, indem mehr Ersparnis notwendig ist, um ein identisches Konsumniveau im Krankheitsfall zu erreichen. Aus diesem Grund wirkt sich der Zinseffekt, der aus einer Verbesserung der Gesundheit folgt, weniger positiv auf die Bildung aus. Im Hinblick auf die übrigen Effekte ist hinge- 
gen keine eindeutige Vergleichbarkeit gegeben, da hier der Einfluss der geänderten Besteuerung im Wesentlichen durch den Verlauf der Nutzenfunktion bestimmt wird.

Für die gesamte Finanzierungssituation der Versicherung bringt ein verbesserter Gesundheitszustand der Versicherten hingegen eindeutig positive Effekte mit sich, denen im Rahmen der individuellen Bildungsentscheidung nicht Rechnung getragen wird. Allgemein spiegeln die Eintrittswahrscheinlichkeiten die Anteile der Versicherten wider, welche die zweite Periode gesund beziehungsweise krank verbringen. Ein Anstieg in der Wahrscheinlichkeit einer zweiten Erwerbsperiode führt bezogen auf alle Versicherten zu einem Anstieg des Erwerbstätigenanteils, so dass eine höhere Zahl an Erwerbseinkommen als Besteuerungsgrundlage dient. Diese Entwicklung führt zum einen zu geringeren Ausgaben im Krankheitsfall, die zu deckende Schadenssumme sinkt entsprechend ab. Zum anderen kann der gleiche Finanzierungsrahmen durch einen geringeren Steuersatz gesichert werden, indem die Zahl der Erwerbseinkommensbezieher ansteigt.

\section{Veränderungen des Steuersatzes $\tau$}

In der vorangegangenen Analyse konnte gezeigt werden, dass eine Veränderung des Steuersatzes der zweiten Periode in einem rein lohnsteuerfinanzierten System ausschließlich die Bildungskapitalerträge betrifft, während die Ersparnisse nicht zusätzlich belastet werden. Hieraus ergaben sich Substitutionseffekte zugunsten der Ersparnisbildung. Derartige Effekte können gemindert werden, indem zusätzlich zum Erwerbseinkommen die Zinseinkünfte besteuert werden, denn eine Gleichbehandlung von Real- und Humankapitalerträgen bringt weniger Möglichkeiten der Steuervermeidung mit sich. ${ }^{151}$

Gleichzeitig wird der positive Einkommenseffekt im Vergleich zu (7.44) verstärkt, d. h. es wird mehr Zeit in die Bildung investiert, um das gewünschte Einkommen bei Gesundheit zu erreichen. Eine Ausweichreaktion auf die Ersparnisse würde nicht zu einer geringeren Steuerbelastung bei Gesundheit führen, die Belastung bei Krankheit hingegen ansteigen lassen, so dass sich hieraus positive Bildungseffekte ergeben können.

Im Hinblick auf den Substitutionseffekt kann hingegen keine eindeutige Aussage getroffen werden. Zwar werden die Lohnersatzleistungen der Versicherung nach wie vor besteuert, so dass die Ersparnisse im Krankheitsfall aufgewertet werden. Die Zinserträge hieraus werden jedoch ebenfalls besteuert, weshalb

${ }^{151} \mathrm{Zu}$ den Auswirkungen eines veränderten Steuersatzes in der zweiten Periode auf die Bildungsinvestitionen siehe die Gleichungen B1 und B2 im Anhang dieses Kapitels. 
letztlich nur die Ersparnissumme der ersten Periode steuerfrei bleibt. Ob sich hieraus eine Abschwächung des Substitutionseffekts ergibt oder ob mehr Ersparnisse gebildet werden, hängt somit entscheidend von der Höhe der Lohnersatzleistungen $\mathrm{ab}$, da diese eine wesentliche Determinante des Sparverhaltens darstellen. Je höher die Lohnersatzleistungen sind, desto eher ist es möglich, dass der Substitutionseffekt abgeschwächt wird, so dass sich die Steuererhöhung weniger stark negativ auf die Bildungsinvestitionen auswirkt.

\subsection{Zusammenfassung}

Jeder Bildungsentscheidung liegt der Trade-off zwischen einem Einkommensverlust in der Gegenwart und zusätzlichem Einkommen in der Zukunft zugrunde. Entscheidet sich ein Individuum dazu, bereits frühzeitig erwerbstätig zu werden, kann ein Teil des erarbeiteten Einkommens für zukünftige Konsumzwecke gespart und somit in die Zukunft transferiert werden. In einer Optimalsituation unter Sicherheit würde ein Individuum genau so lange in die eigene Ausbildung investieren, bis der Grenzertrag der Bildung genau der Verzinsung des Kapitals entspricht. Die Entscheidung für Investitionen in die beiden Kapitalarten - Human- und Realkapital - würde hier ausschließlich anhand der Rendite gefällt werden.

Unsicherheit über die eigene Gesundheit und damit die zukünftige Erwerbsfähigkeit führt dazu, dass mehr Kapital für das Vorsorgesparen aufgewendet wird, um erwartete finanzielle Engpässe bei Krankheit abzumildern, für die es zwei wesentliche Ursachen gibt: Zum einen kommt es zu Einkommensverlusten, wenn eine Erkrankung mit Erwerbsunfähigkeit verbunden ist, woraus sich eine gesteigerte Notwendigkeit der Ersparnisbildung ergibt. Zum anderen müssen sämtliche Gesundheitsausgaben privat finanziert werden, wenn nicht auf die Leistungen einer Versicherung zurückgegriffen werden kann.

Durch die Einführung einer Krankenversicherung kann diese Unsicherheit abgeschwächt werden, indem die Versicherung die Finanzierung medizinischer Leistungen sowie Lohnfortzahlungen im Krankheitsfall gewährleistet. Zugleich wird das Risiko von Bildungsinvestitionen gemindert, da die Bedeutung der Ersparnisse im Krankheitsfall zurückgeht. Folglich wird mehr Bildung nachgefragt. Grundsätzlich kann ein Krankenversicherungssystem dazu beitragen, dass Investitionen in die Bildung aufgrund geringerer Unsicherheit finanziell attraktiver werden. Es erfolgt eine Konsumglättung zwischen den Zuständen krank und damit leistungsbedürftig auf der einen und gesund und arbeitsfähig auf der anderen Seite. 
Die Bildungsanreize einer Krankenversicherung können sich jedoch je nach Finanzierung deutlich voneinander unterscheiden. So bringt eine einkommensunabhängige Prämie keine Verzerrungen mit sich, da diese aus dem Erwerbseinkommen sowie aus den Ersparnissen gleichermaßen finanziert werden kann. In diesem Fall hängt die Entscheidung über die Bildungsinvestitionen einzig von persönlichen Präferenzen sowie der individuellen Risikobereitschaft ab. ${ }^{152}$

Wird die Versicherung wie im System der Gesetzlichen Krankenversicherung in Deutschland ausschließlich über das Lohneinkommen finanziert, während Zinserträge unbelastet bleiben, kann es zu Substitutionseffekten von den Bildungsinvestitionen hin zur Ersparnisbildung kommen. Dieser Effekt wird noch verstärkt, wenn die Leistungen im Krankheitsfall nicht nach dem Äquivalenzprinzip gewährt werden, sondern allen Individuen gleichermaßen zustehen. Individuelle Steuervermeidungseffekte haben in diesem Fall keine Leistungskürzungen zur Konsequenz. Was hier individuell rational erscheint, wirkt sich negativ auf die Einnahmeseite der Versicherung aus und führt so zu steigenden Steuersätzen. Hieraus ergeben sich erneut steigende Belastungen für das Einkommen mit neuerlichen Substitutionseffekten.

Eine Möglichkeit, diesen Negativkreislauf zu beenden, liegt in einem System, in dem auch Zinseinkünfte besteuert werden, um der Ungleichbehandlung von Real- und Humankapital entgegenzuwirken. Nach wie vor ergeben sich jedoch Verzerrungen auf die individuelle Bildungsentscheidung aus der Einkommensbezogenheit der Finanzierung, solange nur die Zinseinkünfte veranlagt werden, nicht aber die gesamten Ersparnisse. ${ }^{153}$ Die Vorteilhaftigkeit gegenüber einer reinen Lohnsteuerfinanzierung wird allerdings eingeschränkt, sobald ein Sparerfreibetrag eingeführt wird, da dieser Zinseinkünfte gegenüber den vollständig zu verbeitragenden Erwerbseinkommen erneut in ihrer Attraktivität steigen lässt. Damit wäre wiederum eine Ungleichbehandlung von Human- und Realkapital zu Lasten der Bildung gegeben.

152 Werden hingegen bei niedrigem Gesamteinkommen steuerfinanzierte Zuschüsse zu den Prämien gewährt, kann es hier ebenfalls zu den bei der Steuerfinanzierung geschilderten Vermeidungsstrategien kommen, auch wenn diese Verzerrungen aufgrund des geringeren Steuervolumens stark abgeschwächt werden. Zudem kann es in diesem Fall sein, dass Bildungsinvestitionen unterlassen werden, um eine Zuschussberechtigung zu erhalten. Dieses Argument lässt sich jedoch nur für diejenigen Individuen anführen, die ein geringes Lebenszeiteinkommen erwarten.

${ }^{153}$ An dieser Stelle sei darauf hingewiesen, dass es nicht im allgemeinen Interesse sein kann, sämtliche Ersparnisse zu besteuern, da diese stets zur Konsumglättung zwischen den Zuständen herangezogen werden müssen. 
Insgesamt ist ein Prämiensystem der Einkommensbesteuerung vorzuziehen, da sich hieraus keinerlei Verzerrungen ergeben. Zudem ist ein solches System weniger anfällig gegenüber Beschäftigungsschwankungen sowie der allgemeinen Einkommensentwicklung. Hingegen ist die gegenwärtige Finanzierung der GKV stark konjunkturabhängig. Zudem bringt die Lohnorientierung eine ausgeprägte Möglichkeit der Beitragsvermeidung mit sich, indem weniger Gewicht auf das Erwerbseinkommen und damit auf die Bildung gelegt wird, sondern vielmehr gegenwärtiges Einkommen über die Ersparnisse in zukünftige Perioden transferiert wird.

Im Hinblick auf Veränderungen der Versicherungsbedingungen ist die Vorteilhaftigkeit jedoch anders zu beurteilen. In einem Prämiensystem wirken sich Erwartungen über Beitragssatzsteigerungen in jedem Fall negativ auf die Akkumulation von Bildungskapital aus, solange ein Individuum risikoavers ist. Aufgrund der Annahme, dass diese Prämie sowohl bei Gesundheit als auch im Krankheitsfall in gleicher Höhe zu leisten ist, nimmt die Bedeutung der Ersparnisse im Krankheitsfall bei einer steigenden Prämie zu, die Bildungsinvestitionen müssen entsprechend sinken.

Zu Substitutionseffekten kann es bei einer rein lohnsteuerfinanzierten Versicherung ebenfalls kommen, da die Erträge des Bildungskapitals bei einer Steuererhöhung sinken, während die Zinserträge unbelastet bleiben. Auf der anderen Seite kann es zu einem Einkommenseffekt kommen, infolge dessen mehr Zeit in die Bildung investiert wird, um die drohenden Einkommensverluste auszugleichen. Welches Verhalten aus individueller Sicht das bessere ist, hängt zum einen vom Zinssatz und zum anderen von den erwarteten Bildungserträgen sowie von der Risikoaversion ab. ${ }^{154}$

Die negativen Auswirkungen einer Anhebung des Steuersatzes sind genau dann am geringsten, wenn beide Kapitalerträge gleichermaßen besteuert werden. In diesem Fall ist nicht nur das Erwerbseinkommen von der Erhöhung betroffen, sondern auch die Zinseinkünfte, so dass sich die Substitutionsmöglichkeiten auf die Ersparnissumme selbst, nicht aber auf die Erträge beschränken. Hiermit einher geht auch ein stärkerer Einkommenseffekt, da der Anreiz steigt, das geringere Nettoeinkommen durch ein höheres Bruttoeinkommen auszugleichen.

\footnotetext{
${ }^{154}$ Ein starker Anstieg des Beitragssatzes scheint im gegenwärtigen GKV-System unumgänglich, da die Erwerbstätigkeit aufgrund einer alternden Bevölkerung sowie steigender Arbeitslosigkeit zurückgeht. Die Beitragssatzprognosen für die GKV reichen von $23,1 \%$ bis zu $39,5 \%$ im schlechtesten Fall (vgl. Postler (2003), S. 23).
} 


\subsection{Anhang zu Kapitel 7.4.3}

A Einfluss der Gesundheit auf die Bildungsentscheidung

Bilden des Totalen Differentials aus (7.49) und (7.50):

$$
\frac{d b_{S}}{d h}=-\frac{D_{h}}{D_{b}}
$$

mit

$$
\begin{aligned}
D_{h} & \equiv \frac{1-\tau}{1-t} \beta^{\prime}\left[\pi^{\prime} \alpha+\pi \alpha^{\prime}\right] u_{2 G, S}^{\prime} \\
& +\pi^{\prime}(1+(1-\tau) r)\left[u_{2 K, S}^{\prime}-u_{2 G, S}^{\prime}\right] \\
& +\pi\left[\frac{1-\tau}{1-t} \alpha \beta^{\prime}-(1+(1-\tau) r)\right](1-\tau) \alpha \beta^{\prime} w u_{2 G, S}^{\prime \prime}, \\
D_{b} & \equiv \pi \frac{1-\tau}{1-t} \alpha \beta^{\prime \prime} u_{2 G, S}^{\prime} \\
& +\pi\left[\frac{1-\tau}{1-t} \alpha \beta^{\prime}-(1+(1-\tau) r)\right](1-\tau) \alpha \beta^{\prime} w u_{2 G, S}^{\prime \prime}<0 .
\end{aligned}
$$

B Bedeutung einer erwarteten Steuersatzänderung in der zweiten Periode

$$
\begin{aligned}
& \frac{d b_{\tau}}{d \tau}=-\frac{D_{\tau}}{D_{b}} \\
& D_{\tau} \equiv-\frac{\pi \alpha \beta^{\prime}}{(1-t)(1+(1-\tau) r)} u_{2 G, S}^{\prime} \\
& \quad-\pi\left[\frac{1-\tau}{1-t} \alpha \beta^{\prime}-(1+(1-\tau) r)\right](r S+(1+\varphi) w) u_{2 G, S}^{\prime \prime} \\
& \quad+(1-\pi)(1+(1-\tau) r)(r S+q w) u_{2 K, S}^{\prime \prime}
\end{aligned}
$$

Der Nenner von (B1) bleibt zu (A1) unverändert. 


\section{Empirische Analyse der Gesundheitsdeterminanten anhand von Mikrodaten}

\subsection{Querschnittsanalyse}

Wie in den vorangegangenen Kapiteln gezeigt wurde, ist eine gute Gesundheit für dauerhaftes Wirtschaftswachstum unverzichtbar. Zum einen folgen aus der Gesundheit direkte Produktivitätseffekte, indem sich die physische sowie die mentale Leistungsfähigkeit erhöht und Fehlzeiten gesenkt werden können, woraus sich eine optimale Nutzung des Produktionsfaktors Arbeit ergibt. Zum anderen ist eine gute Gesundheit für die Bildungskapitalakkumulation förderlich, da die Lerneffizienz sowie die Rendite der Bildungsinvestitionen gesteigert werden, so dass der Gesundheit eine große Bedeutung für die wirtschaftliche Entwicklung beigemessen werden muss.

In der nachfolgenden empirischen Untersuchung wird analysiert, welche Faktoren das gesundheitsrelevante Verhalten sowie den eigentlichen Gesundheitszustand beeinflussen. Zunächst wird das gesundheitsrelevante Verhalten von Individuen untersucht, indem Merkmale wie Rauchen und Körpergewicht zu einem ordinal skalierten Verhaltensindikator zusammengefasst werden. Gemäß den theoretischen Überlegungen hierzu ist zu vermuten, dass das verfügbare Einkommen, das Bildungsniveau sowie die Arbeitszeit relevante Einflussfaktoren darstellen. Darüber hinaus ist von Interesse, inwiefern sich das Verhalten auf das gesundheitliche Wohlbefinden auswirkt und ob sich zusätzliche direkte Gesundheitseinflüsse aus der Beschäftigung ergeben. Hierzu wird die Selbsteinschätzung des Gesundheitszustandes als Indikator verwendet. Die Antwortskala reicht von 1 (schlechter gegenwärtiger Gesundheitszustand) bis 5 (sehr guter Gesundheitszustand), so dass für beide relevanten Fragestellungen ein Schätzverfahren für kategoriale Variablen benötigt wird.

Zudem handelt es sich sowohl beim gesundheitsrelevanten Verhalten als auch bei der Gesundheit um latente, d. h. nicht beobachtbare, stetige Variablen. In einem solchen Fall wird der Übergang von einer Kategorie zur nächsten durch das Überschreiten eines nicht beobachtbaren Schwellenwertes modelliert (siehe hierzu die Erläuterungen im Anhang des Kapitels).

Würde man nun auf diese Variablenstruktur das Verfahren der linearen Regression anwenden, würden folgende Probleme auftreten: Erstens würde die Regressionsgerade insbesondere bei sehr wenigen Kategorien nahezu waagerecht verlaufen. Zweitens sind die Fehlerterme heteroskedastisch und nicht normalverteilt. Zudem lässt sich drittens allgemein sagen, dass die Ergebnisse des Modells für geordnete Kategorien nur dann mit denen des linearen Regressionsmodells 
vereinbar sind, wenn die Abstände zwischen den Kategorien überall exakt gleich sind. Dies ist bei latenten Variablen keineswegs zwingend der Fall, da es sich jeweils um subjektive Entscheidungen und Einschätzungen handelt, bei denen eine Gleichverteilung der Schwellenabstände nicht überprüft werden kann. Zudem ist davon auszugehen, dass eine exakte Gleichverteilung zufällig wäre. Bei einer Anwendung des linearen Wahrscheinlichkeitsmodells müsste entsprechend von inkorrekten Ergebnissen ausgegangen werden, so dass in der vorliegenden Arbeit das Ordered Probit-Modell angewendet wird.

\subsubsection{Datenmaterial und Hypothesen}

Zur Analyse des gesundheitsrelevanten Verhaltens sowie der Selbsteinschätzung des persönlichen Gesundheitszustandes werden Daten des Sozioökonomischen Panels (SOEP) verwendet. Hierbei handelt es sich um Individual- und Haushaltsdaten, die vom deutschen Institut für Wirtschaftsforschung seit 1984 im jährlichen Rhythmus erhoben werden. Der Datensatz wurde auf Individuen im erwerbsfähigen Alter (unter 65 Jahren) beschränkt, um eine Vergleichbarkeit hinsichtlich möglicher Arbeitszeiteffekte zu gewährleisten. In der Welle 2004 sind Sport, Rauchen und das Körpergewicht als Verhaltensvariablen sowie die Selbsteinschätzung des Gesundheitszustandes als Gesundheitsindikator vorhanden, so dass sich die Verwendung dieser Welle für die nachfolgende Querschnittsanalyse anbietet.

Im Rahmen der theoretischen Modellanalyse werden drei wesentliche Effekte der Arbeitszeit auf die Gesundheit postuliert. Es wird erstens ein Einkommenseffekt der Arbeitszeit unterstellt, indem mit steigender Arbeitszeit bei gegebenem Lohnsatz auch das Erwerbseinkommen ansteigt. Zweitens kommt es zu einem Zeiteffekt, da mit steigender Arbeitszeit immer weniger Zeit für Gesundheitsinvestitionen zur Verfügung steht. Darüber hinaus existiert drittens ein $\mathrm{Ab}$ schreibungseffekt, wenn übermäßig viel Arbeit mit Stress verbunden ist, so dass sich c. p. eine Verschlechterung der individuellen Gesundheit einstellt.

Folgende Hypothesen werden untersucht:

Hypothese 1: Mit steigendem Einkommen steigen auch die Gesundheitsinvestitionen.

Für das individuelle Gesundheitsverhalten ergeben sich aus diesen Zusammenhängen folgende Implikationen: Aufgrund der umfassenden medizinischen Versorgung in Deutschland ist der Konsum verordneter medizinischer Leistungen nur begrenzt einkommensabhängig, indem beispielsweise Zuzahlungen zu Arzneimitteln geleistet oder bestimmte Heil- und Hilfsmittel selbst finanziert werden müssen. Darüber hinaus spiegelt der Einkommenseffekt insbesondere die 
Nachfrage nach privaten Gesundheitsleistungen wider, denn je höher das individuell verfügbare Einkommen ist, desto mehr Geld kann auch für Gesundheitsinvestitionen aufgewendet werden, die nicht direkt medizinischen Leistungen zuzurechnen sind. Hierzu zählt insbesondere der Konsum von hochwertigen und damit häufig teureren Nahrungsmitteln, so dass das Normalgewicht den Einkommenseffekt mit abbildet. ${ }^{155}$

Hypothese 2: Zwischen der individuellen Arbeitszeit und dem gesundheitsrelevanten Verhalten besteht ein konkaver Zusammenhang.

Einen negativen Partialeffekt bringt die verminderte Investitionszeit mit sich. Da aus dem Datenmaterial nicht hervorgeht, ob es sich bei Arztbesuchen um Vorsorgeuntersuchungen oder um kurative Maßnahmen handelt, wird diese Variable nicht als Merkmal gesundheitsrelevanten Verhaltens herangezogen. Stattdessen wird sportliche Betätigung in den Verhaltensindikator aufgenommen, da Sport mit hohem Zeitaufwand verbunden ist. Gleichzeitig kann die Sportvariable ebenfalls einen Teil des Einkommenseffektes abbilden, wenn die gewählte Sportart mit entsprechenden Kosten, z. B. bezüglich der Ausrüstung, verbunden ist.

Zum dritten gibt es einen direkten Effekt der Arbeit auf das Gesundheitsverhalten, indem arbeitsbedingter Stress häufig mit einer ungesunden Lebensweise einhergeht. Als Indikator hierfür kommt wiederum das Normalgewicht in Frage, da Stress mit ungesunder Ernährung verbunden sein kann. Darüber hinaus stehen in der verwendeten Welle Daten darüber zur Verfügung, ob es sich bei der befragten Person um einen Raucher handelt. Positive Effekte wie Zufriedenheit durch Selbstverwirklichung oder Anerkennung, die die Abschreibungsrate mindern, können hierüber jedoch nicht abgebildet werden.

Nimmt man diese Arbeitszeiteffekte zusammen, so ist ein konkaver Zusammenhang zu erwarten: Zunächst überwiegen die positiven Aspekte der Arbeit. Es steht mehr Einkommen für Investitionen zur Verfügung, und die Opportunitätskosten einer Krankheit nehmen mit steigendem Einkommen zu. Folglich ist anzunehmen, dass die Investitionen in die Gesundheit mit zunehmender Arbeitszeit ansteigen. Nimmt darüber hinaus das Stressempfinden mit der Arbeitszeit zu und bleibt darüber hinaus nur wenig Freizeit, verkehrt sich der Einfluss ins Negative, die Gesundheitsinvestitionen nehmen ab.

${ }^{155}$ Es ist daruber hinaus davon auszugehen, dass das Normalgewicht maßgeblich durch körperliche Betătigung beeinflusst wird, wobei auch hier ein Einkommensbezug besteht (vgl. Hypothese 2). 
Hypothese 3: Auch zwischen der Arbeitszeit und dem individuellen Gesundheitszustand wird ein konkaver Zusammenhang postuliert, da Stressfaktoren sowie körperliche Anstrengung mit zunehmender Arbeitszeit an Bedeutung gewinnen.

Nun ist gesundheitsrelevantes Verhalten nicht automatisch mit Gesundheit per se gleichzusetzen, da der Verhaltensindikator nur einen Teil der Gesundheitseinflüsse abdeckt. Insofern ist weiterhin zu untersuchen, wie sich das Verhalten auf die Selbsteinschätzung des individuellen Gesundheitszustandes auswirkt und welche direkten Effekte sich aus der Arbeitszeit ergeben, die nicht aus den Verhaltensvariablen abgeleitet werden können. Hierunter fallen sowohl positive Einflussfaktoren wie ein hohes $\mathrm{Ma} ß$ an Selbstbestimmung und soziale Kontakte, als auch negative Effekte, die nicht über die Verhaltensindikatoren abgebildet werden, so z. B. körperliche Anstrengung.

\subsubsection{Variablenbeschreibung}

\section{Abhängige Variablen}

Die abhängige Variable der ersten Schätzung ist ein Indikator des gesundheitsrelevanten Verhaltens. Dieser setzt sich aus den einzelnen Variablen Sport, Normalgewicht sowie Rauchen wie folgt zusammen: Zunächst wird aus dem Körpergewicht und der Körpergröße der Individuen der Body Mass Index (nachfolgend BMI) berechnet. Hierzu wird die Körpergröße, angegeben in Metern, quadriert. Anschließend wird das Gewicht in Kilogramm durch die quadrierte Körpergröße geteilt. Eine Person befindet sich im normalgewichtigen Bereich, wenn der BMI zwischen 18,5 und 24,9 liegt (vgl. WHO / World Health Organization (2003), S. 69).

Eine wesentliche Kritik an der Verwendung dieser Normalgewichtsdefinition besteht in der Altersunabhängigkeit. Tatsächlich lässt sich zeigen, dass die für die Lebenserwartung optimale Relation von Gewicht und Größe mit dem Alter zunimmt (vgl. National Research Council (1989), S. 564). Die nachfolgende Tabelle 3 gibt daher einen Überblick über die Einteilung altersabhängiger BMIKlassen. Hier fällt insbesondere auf, dass die Höchstgrenze für die Altersgruppe der 19-24jährigen gleichzeitig die Untergrenze für die über 65jährigen darstellt. $^{156}$

${ }^{156}$ Durch die Altersbereinigung werden insgesamt 12,78\% der Personen (15,64\% der Männer, $9,64 \%$ der Frauen) als normalgewichtig eingestuft, bei denen ansonsten ein Übergewicht festgestellt würde. 
Tabelle 3: Wünschenswerter Body Mass Index im Verhältnis zum Alter

\begin{tabular}{|c|c|}
\hline Altersgruppe & BMI \\
\hline $19-24$ & $19-24$ \\
\hline $25-34$ & $20-25$ \\
\hline $35-44$ & $21-26$ \\
\hline $45-54$ & $22-27$ \\
\hline $55-64$ & $23-28$ \\
\hline$>=65$ & $24-29$ \\
\hline
\end{tabular}

Quelle: Vgl. National Research Council (1989), S. 564.

Damit ergeben sich für die Variable Gesundheitsverhalten folgende Kategorien:

0 Die Person treibt nicht regelmäßig Sport, raucht und hat ein Gewicht, das vom Normalgewicht abweicht.

1 Eine der Bedingungen Sport, Nichtraucher und Normalgewicht ist erfüllt

2 Zwei der Bedingungen Sport, Nichtraucher und Normalgewicht sind erfüllt

3 Die Person treibt regelmäßig Sport, ist Nichtraucher und hat Normalgewicht

Die zweite Schätzung befasst sich nicht mit dem gesundheitsrelevanten Verhalten, sondern mit der Gesundheit per se. Analog zu Nocera und Zweifel (1998) wird die wird auf die Selbsteinschätzung des Gesundheitszustandes als Gesundheitsindikator zurückgegriffen. Hier reichen die Kategorien von einer 1 für einen schlechten Gesundheitszustand bis zu einer 5 furr die Einschätzung eines sehr guten Zustandes.

\section{Erklärende Variablen}

Die erklärenden Variablen lassen sich in vier Kategorien einteilen. In der ersten Kategorie der prädisponierenden Variablen sind alle diejenigen Variablen enthalten, die unweigerlich mit der Person verknüpft sind und daher als unveränderliche individuelle Merkmale angesehen werden können. Hierzu gehören das Alter und das Geschlecht ebenso wie die Bildung oder die Nationalität. Die zweite Kategorie bildet die sozioökonomischen Variablen ab, die den Familienstand, das Einkommen sowie den Versicherungsstatus betreffen. In der dritten 
Kategorie werden individuelle Gesundheitsmerkmale aufgelistet, während die vierte Kategorie letztlich die Arbeitszeitvariablen umfasst. Eine Zusammenfassung aller verwendeten Variablen liefert Tabelle 4.

Tabelle 4: Verwendete Variablen in der Querschnittsanalyse

\begin{tabular}{|ll|}
\hline Pri̊dispon. Variablen & \\
Alter & Alter in Jahren \\
Alter & Alter quadriert \\
Geschlecht & 1, wenn weiblich \\
Interaktion & Alter * Geschlecht \\
Realschule & 1, wenn Realschulabschluss \\
FH-Reife & 1, wenn Fachhochschulreife \\
Abitur & 1, wenn Abitur \\
Diplom & 1, wenn Diplom \\
Ausländer & 1, wenn Ausländer \\
Behinderung & 1, wenn Erwerbs- oder Schwerbehinderung \\
Soziobk. Variablen & \\
Partner_HH & 1, wenn Partner im Haushalt lebt \\
Kind & 1, wenn mind. 1 Kind unter 16 Jahren im Haushalt \\
& lebt \\
Ln_HH-Netto & Logarithmiertes Haushaltsnettoeinkommen \\
Eink.-Sorgen & 1, wenn große Einkommenssorgen \\
PKV & 1, wenn PKV-versichert \\
PKV_Zusatz & 1, wenn private Zusatzversicherung \\
Gesundheitsmerkmale & \\
Gesundverhalten & Generiertes Gesundheitsverhalten (0 - sehr unge- \\
Gesundzustand & sunder Lebensstil bis 3 - sehr gesunder Lebensstil) \\
Anstrengung & Gesundheitszustand gegenwärtig (1 - schlecht bis \\
& 5- sehr gut) \\
Arztbesuche & 1, wenn Gesundheit anstrengende Tätigkeiten \\
KH (Vj.) & stark erschwert \\
AU (Vj.) & Zahl der Arztbesuche im letzten Quartal \\
Ln_BMI & 1, wenn im Vorjahr Krankenhausaufenthalt \\
(Ln_BMI) & Tage im Vorjahr wegen Krankheit nicht gearbeitet \\
Sport & Logarithmierter Body Mass Index \\
Rauchen & Logarithmierter Body Mass Index quadriert \\
Arbeitsmerkmale & 1, wenn regelmäßig Sport \\
Arbeitszeit & 1, wenn gegenwärtig Raucher \\
Arbeitszeit & \\
& Tatsächliche Arbeitszeit \\
& Tatsächliche Arbeitszeit quadriert \\
\hline
\end{tabular}




\section{Prädisponierende Variablen}

Sowohl für das Alter als auch für das Geschlecht können keine eindeutigen Vorhersagen für den Einfluss auf die Gesundheit und das Gesundheitsverhalten getroffen werden. Es ist a priori nicht klar, ob sich ältere Menschen weniger bewegen und entsprechend weniger Sport treiben, was sich negativ auf den Verhaltensindikator auswirken würde, oder aber ob die mit dem Alter einhergehende höhere Morbidität zu einem gesteigerten Gesundheitsbewusstsein und damit zu einem besseren gesundheitsrelevanten Verhalten führt. Für die Einschätzung des Gesundheitszustandes wird aufgrund der höheren Erkrankungswahrscheinlichkeit aber ein eindeutig negatives Vorzeichen erwartet. Der quadrierte Altersterm soll zudem Aufschluss darüber geben, wie sich die Einschätzung mit dem Alter verändert.

Da Frauen im Durchschnitt häufiger den Arzt aufsuchen als Männer, ist damit zu rechnen, dass diese der Gesundheit eine höhere Wertschätzung beimessen als Männer. Auf der anderen Seite ist es jedoch ebenso möglich, dass Frauen über einen schlechteren Gesundheitszustand verfügen, weshalb vermehrt kurative Leistungen in Anspruch genommen werden müssen. Für das Verhalten ist jedoch ebenfalls keine eindeutige Aussage möglich. Die Überlegung, ob sich das Alter bei Frauen anders auf die Gesundheit auswirkt als bei Männern, wird durch den Interaktionsterm abgedeckt. Dieser nimmt nur für Frauen Werte gröBer Null an, weshalb hierdurch der geschlechtsspezifische Einfluss des Alters beobachtet werden kann. Zumindest für den selbst eingeschätzten Gesundheitszustand ist hier ebenfalls ein positiver Einfluss zu erwarten.

Die nachfolgenden vier Variablen aus Tabelle 4 geben Aufschluss über die Schulbildung der befragten Personen. Der Einfluss der Bildung wird durch vier Dummy-Variablen abgebildet, wobei Personen, die maximal über einen Hauptschulabschluss verfügen, als Referenzgruppe dienen. In Bezug auf das gesundheitsrelevante Verhalten ist zu erwarten, dass dieses positiv durch die Bildung beeinflusst wird. Es ist davon auszugehen, dass Kenntnisse über die Gesundheit beeinflussende Faktoren mit dem Stand der Bildung zunehmen. Diese Annahme betrifft sowohl die Gefahren des Rauchens als auch Informationen über die Ernährung und die Notwendigkeit, Sport zu treiben. Zudem steigt mit der Bildung in der Regel auch das Erwerbseinkommen, weshalb die Opportunitätskosten einer schlechten Gesundheit ansteigen. Da im Verhältnis zur Referenzgruppe für alle Bildungsdummys ein positives Vorzeichen zu erwarten ist, lassen sich die unterschiedlichen Effekte lediglich über die Koeffizienten abbilden. Somit ist der Einfluss der Bildung umso größer, je größer auch die entsprechenden Koeffizienten ausfallen. 
Im Hinblick auf den selbst eingeschätzten Gesundheitszustand kann hingegen keine eindeutige Prognose abgegeben werden. Zwar lässt die Theorie einer gesteigerten Allokations- und Produktionseffizienz einen positiven Einfluss der Bildung vermuten. Dem kann jedoch entgegengestellt werden, dass mit zunehmender Kenntnis über den „optimalen“ Gesundheitszustand die eigene Gesundheit eher unterschätzt und damit als zu schlecht eingestuft wird.

Zuletzt sind die Variablen Ausländer und Behinderung dieser Kategorie zuzuordnen. Die Bedeutung der Nationalität ist nicht abzusehen, während bei der Variable Behinderung für beide Gesundheitsindikatoren ein negatives Vorzeichen zu erwarten ist. Diese Annahmen können dadurch begründet werden, dass die sportliche Betätigung durch die Behinderung eingeschränkt sein kann, was sich auch auf das Körpergewicht negativ auswirkt. Es ist darüber hinaus nahe liegend, dass Behinderte ihre eigene Gesundheit schlechter einschätzen als Personen ohne Behinderung.

\section{Sozioökonomische Variablen}

Die folgenden zwei Variablen betreffen die Bedeutung der familiären Situation für die Gesundheit. Ein Kind im Haushalt kann sich sowohl positiv als auch negativ auf das Gesundheitsverhalten auswirken. Ein positiver Partialeffekt würde sich dann ergeben, wenn Personen mit Kindern im Haushalt beispielsweise seltener rauchen. Durch den hohen Zeitaufwand, den Kinder in der Regel mit sich bringen, steht c. p. jedoch auch weniger Zeit für sportliche Betätigung zur Verfügung, so dass diesem Partialeffekt ein negativer Einfluss zuzuschreiben ist. Hinsichtlich des Körpergewichts sind Effekte in beide Richtungen denkbar, da zum einen die Notwendigkeit regelmäßiger Mahlzeiten prinzipiell eine gesündere Ernährung bedingt, zum anderen mangelnder Sport aber negative Auswirkungen auf den BMI besitzt. Gleichwohl ist es ebenso möglich, dass sich Familien mit Kindern häufiger bewegen, ohne dass diese Bewegung direkt sportlicher Betätigung zuzurechnen ist. Für den Gesamteffekt ist damit entscheidend, welche Partialeffekte überwiegen. Eine ähnliche Argumentation bezüglich der Richtung der Effekte ist mit der Variable Partner_HH verbunden, auch wenn die Ausprägung jeweils geringer sein dürfte.

Als Einkommensvariablen werden das logarithmierte Haushaltsnettoeinkommen sowie Einkommenssorgen gewählt. Das Haushaltseinkommen ist auf positive Werte beschränkt, gleichzeitig nach oben jedoch offen. Sehr hohe Einkommen sind insgesamt seltener, da zum einen der Bedarf für entsprechend dotierte Jobs relativ gering ist und zum anderen Lohnerhöhungen in der Regel prozentual erfolgen, weshalb die Einkommen in der Zeit exponentiell ansteigen. Damit weist das Haushaltseinkommen eine Rechtsschiefe auf. Das Problem einer solchen 
Verteilung ist, dass diese verzerrte Schätzergebnisse liefert, da Werte, die um den Mittelwert schwanken, unterschätzt werden. Durch das Logarithmieren wird dieser multiplikative Prozess, dem die Lohnentwicklung unterliegt, in einen additiven Zusammenhang überführt, so dass sich die Verteilung an eine Normalverteilung angleicht. Bei Variablen, die einer solchen Struktur unterliegen, spricht man allgemein von einer Logarithmischen Normalverteilung. ${ }^{157}$

Für die Bereitschaft der Individuen, in ihre Gesundheit $\mathrm{zu}$ investieren, ist ein positiver Einfluss des Einkommens zu erwarten. Hierfür spricht zum einen, dass insbesondere Nahrungsmittel mit einer besseren Qualität gekauft werden können, was sich positiv auf das Normalgewicht auswirken würde. Zudem sind die Einkommensverluste aus einer Krankheit umso höher, je höher das Erwerbseinkommen ist. An dieser Stelle sei darauf hingewiesen, dass hier bewusst nicht das individuelle Erwerbseinkommen, sondern das Haushaltsnettoeinkommen verwendet wird, um sonstige Vermögen sowie das Einkommen des Partners nicht auszuklammern. Darüber hinaus besteht eine positive Korrelation zwischen Einkommen und Schulbildung, so dass der Bildungsstand letztlich über das Einkommen erneut auf das Gesundheitsverhalten wirkt. Den selbst eingeschätzten Gesundheitszustand betreffend ist aufgrund der geringen Einkommensbezogenheit des deutschen Gesundheitswesens von einem positiven oder neutralen Effekt auszugehen.

Über den eigentlichen Einkommenseffekt hinaus wird zudem untersucht, wie sich finanzielle Sorgen auf die Gesundheit auswirken. Es ist denkbar, dass auch ein hohes Nettoeinkommen nicht für Gesundheitsinvestitionen verwendet werden kann, wenn diesem entsprechend hohe Verbindlichkeiten gegenüberstehen. Zudem können finanzielle Sorgen psychischen Stress verursachen, der sich entsprechend auf das persönliche Wohlbefinden und damit auf die Einschätzung des Gesundheitszustandes auswirkt.

Für die Versicherungsvariable $P K V$ wird für beide Schätzungen ein positiver Einfluss erwartet, da in der Regel mehr Gesundheitsleistungen durch die Versicherung finanziert werden. Zum anderen verhindert die Versicherungspflichtgrenze, dass sich jede Person privat versichern kann, so dass letztlich auch hier das Einkommen wieder eine Rolle spielt. ${ }^{158}$ Gleiches ließe eine private Zusatzversicherung vermuten. Nichts desto trotz ist es ebenso möglich, dass sich ge-

\footnotetext{
157 Năhere Informationen zur Logarithmischen Normalverteilung finden sich bei Aitchison und Brown (1976).

${ }^{158}$ Zudem wird die Option, sich privat zu versichern, insbesondere von niedrigen Risiken wahrgenommen, da in der PKV risikoorientierte Prämien erhoben werden (vgl. Breyer et al. (2005), S. 199.
} 
rade diejenigen privat zusatzversichert haben, die bereits über einen eher schlechten Gesundheitszustand verfügen oder aber bei denen die Bereitschaft zu präventiven Gesundheitsinvestitionen gering ist, so dass zusätzliche medizinische Leistungen in Anspruch genommen werden. Damit kann nicht eindeutig prognostiziert werden, ob sich eine private Zusatzversicherung tatsächlich positiv auf die Gesundheitsindikatoren auswirkt.

\section{Gesundheitsmerkmale}

Die Dummyvariable Anstrengung gibt Aufschluss darüber, ob eine deutliche Gesundheitsbeeinträchtigung vorliegt, die nicht mit einer Behinderung gleichzusetzen ist. Es stellt sich hierbei die Frage, inwiefern sich eine solche Beeinträchtigung auf das Gesundheitsverhalten auswirkt. Hinsichtlich des Vorzeichens besteht keine eindeutige Vermutung. Zum einen kann es sein, dass der Anreiz zu einem gesunden Lebensstil erhöht wird, um der gesundheitlichen Schwäche entgegenzuwirken. Zum anderen ist es jedoch ebenso möglich, dass die Beeinträchtigung nicht mit dem Lebensstil in Verbindung gebracht wird oder dass keine Bereitschaft zu einer Verhaltensänderung besteht. In diesem Fall ergäbe sich kein eindeutiger Effekt. Hinsichtlich der Selbsteinschätzung der Gesundheit wird jedoch ein eindeutig negatives Vorzeichen erwartet.

Die zwei Variablen Arztbesuche und $K H(V j$. $)$ geben Aufschluss darüber, wie sich die Inanspruchnahme medizinischer Leistungen auf das Gesundheitsverhalten einerseits sowie auf die Gesundheit andererseits auswirkt. Während bei Krankenhausaufenthalten zu erwarten ist, dass diese sich negativ auf das Verhalten sowie ebenfalls negativ auf den Gesundheitszustand auswirken, liefert die Theorie für die Zahl der Arztbesuche keine eindeutige Prognose, da es hier wiederum zu gegenläufigen Effekten kommen kann. Für ein positives Vorzeichen in der Verhaltensgleichung spricht die Annahme, dass es sich hierbei um gesundheitsbewusste Personen handelt, die Vorsorgeuntersuchungen im Sinne der Gesundheitsinvestitionen in Anspruch nehmen und die die Anweisungen der Ärzte hinsichtlich einer gesunden Lebensweise auch entsprechend befolgen. Damit wären die Arztbesuche als Komplemente zu den eigenen Bemühungen zu sehen. Ein negatives Vorzeichen würde hingegen dafür sprechen, dass Arztbesuche Substitute darstellen. In diesem Fall geht die Bereitschaft, selbst etwas für die Gesundheit zu tun, zurück, sobald derartige Gesundheitsleistungen konsumiert werden. Im Hinblick auf den Gesundheitszustand wird ein negatives Vorzeichen erwartet, d. h. je mehr Arztbesuche getätigt werden, desto wahrscheinlicher ist es, dass die eigene Gesundheit als schlecht eingeschätzt wird.

Aufgrund der Abhängigkeit der Gesundheit von der Investitionsbereitschaft der Individuen ist darüber hinaus zu erwarten, dass ein schlechter Gesundheitszu- 
stand langfristig mit negativen Folgen verbunden ist. So können sich Krankheiten und Verletzungen, die stationär behandelt werden mussten, beispielsweise negativ auf die körperliche Belastbarkeit auswirken, wenn keine vollständige Heilung erzielt werden konnte. Da hierdurch gleichzeitig das Körpergewicht negativ beeinflusst wird, ist von einem negativen Einfluss der Krankenhausbesuche aus dem Vorjahr auf die abhängige Variable auszugehen. Ein negatives Vorzeichen wird zudem für die Selbsteinschätzung des Gesundheitszustandes postuliert.

Als letztes Gesundheitsmerkmal ist die Arbeitsunfähigkeit im Jahr 2003 zu nennen. Diese Variable soll langfristige Effekte von schweren Erkrankungen abbilden, die zu Fehlzeiten geführt haben. Während der Einfluss auf das Gesundheitsverhalten a priori nicht bestimmbar ist, ist zu vermuten, dass sich diese Variable negativ auf den Gesundheitszustand auswirkt.

In der Gesundheitszustandsgleichung sind darüber hinaus die Variablen Ln_BMI, (Ln_BMI) ${ }^{2}$, Sport und Rauchen enthalten. Sowohl Körpergröße als auch das Gewicht können durch die Lognormalverteilung gut abgebildet werden, da sie keine negativen Werte annehmen können (vgl. Johnson et al. (1994), S. 239). Zudem handelt es sich um Variablen, deren Verteilung rechtsschief ist. Aus diesen Gründen wird für den BMI, wie beim Haushaltsnettoeinkommen auch, die logarithmierte Form verwendet. Gemäß der Definition für die Normalgewichtsklassen des BMI ist zu erwarten, dass es zu einem konkaven Zusammenhang kommt: Mit steigendem BMI verbessert sich auch die Wahrscheinlichkeit für einen guten Gesundheitszustand, allerdings mit abnehmender Rate, so dass für den quadrierten Term ein negativer Einfluss postuliert wird. Die erwarteten Vorzeichen sind damit für den BMI positiv sowie negativ für den quadrierten Term.

Aufgrund der gesundheitsschädigenden Wirkung des Rauchens wird hier ein negativer Zusammenhang unterstellt, während davon ausgegangen wird, dass sich regelmäßige sportliche Betätigung positiv auf den Gesundheitszustand auswirkt. Zwar können durch Sport auch Verletzungen auftreten, es besteht jedoch die Vermutung, dass diese durch die positiven Einflüsse überkompensiert werden.

\section{Arbeitsmerkmale}

Ein zentrales Ergebnis des theoretischen Teils dieser Arbeit ist der konkave $\mathrm{Zu}$ sammenhang zwischen den Faktoren Arbeitszeit und Gesundheit. Da sich ein wesentlicher Teil der Argumentation auf die Investitionen in die Gesundheit bezieht, wird hier von einem positiven Vorzeichen für die Variable Arbeitszeit sowie von einem negativen für die quadrierte Arbeitszeit ausgegangen. In einem 
solchen Fall würde sich die Wahrscheinlichkeit eines gesunden Lebenswandels mit zunehmender Arbeitszeit zwar zunächst erhöhen, jedoch nur so lange, bis die Stressfaktoren und der Zeitaufwand Überhand nehmen, so dass der Einkommenseffekt nicht mehr ausreicht, um die negativen Auswirkungen auszugleichen.

Weiterhin wird davon ausgegangen, dass nicht alle Arbeitszeiteffekte über die für das gesundheitsrelevante Verhalten gewählten Indikatoren abgebildet werden. Vielmehr ist auch ein direkter positiver Effekt auf die Gesundheit zu vermuten, der sich jedoch ebenfalls mit zunehmender Arbeitszeit abschwächt.

\subsubsection{Deskriptive Statistik}

Die für die erste Schätzung verwendeten Daten aus dem Jahr 2004 umfassen insgesamt 4765 Personen im Alter von 17 bis unter 65 Jahren, da diese das gesetzliche Renteneintrittsalter noch nicht erreicht haben und somit als grundsätzlich erwerbsfähig eingestuft werden können. Insbesondere im Hinblick auf die Gesundheit sowie die Erwerbstätigkeit können sich geschlechtsspezifische Unterschiede ergeben, weshalb die Schätzungen jeweils noch einmal allein für Männer sowie für Frauen durchgeführt werden. Der Stichprobenumfang beträgt hier 2494 bzw. 2271 Personen. Die nachfolgende Tabelle 5 zeigt jeweils den Mittelwert sowie die Standardabweichung der verwendeten Variablen.

In der für die erste Schätzung verwendeten Gesamtstichprobe sind 47,7\% der beteiligten Personen Frauen. Das Durchschnittsalter liegt für alle verwendeten Stichproben bei knapp über 39 Jahren. Deutliche Unterschiede zwischen Männern und Frauen treten bei der Schulbildung auf. Insgesamt $71,2 \%$ der Frauen haben mindestens einen Realschulabschluss, aber nur 63,5\% der Männer. Da sich die durchschnittliche Zahl derer, die zumindest die Fachhochschulreife haben, nicht wesentlich zwischen den Geschlechtern unterscheidet, ist der Bildungsunterschied insbesondere auf den Realschulabschluss zurückzuführen.

Das durchschnittliche Haushaltsnettoeinkommen liegt bei Männern mit 3009 Euro etwas höher als bei Frauen (2938 Euro), obwohl Frauen in dieser Stichprobe durchschnittlich über den höheren Schulabschluss verfügen. Zudem liegt das Maximum für Frauen bei 12700 Euro, während es bei der Gruppe der Männer mit 15690 Euro deutlich darüber liegt. Dementsprechend ist auch das logarithmierte Haushaltsnettoeinkommen bei Männern höher als bei Frauen. Eine Erklärung hierfür liefert unter anderem die tatsächliche Arbeitszeit, die mit durchschnittlich fast 31 Stunden für Frauen deutlich unter der Arbeitszeit der Männer liegt, die nahezu 40 Stunden im Durchschnitt beträgt. 
Tabelle 5: Deskriptive Statistik, Querschnittsanalyse

\begin{tabular}{|c|c|c|c|c|c|c|}
\hline & \multicolumn{2}{|c|}{$\begin{array}{r}\text { Gesamt } \\
N=4765\end{array}$} & \multicolumn{2}{|c|}{$\begin{array}{l}\text { Männer } \\
\mathrm{N}=2494\end{array}$} & \multicolumn{2}{|c|}{$\begin{array}{c}\text { Frauen } \\
\mathrm{N}=2271\end{array}$} \\
\hline & $\begin{array}{c}\text { Mittel- } \\
\text { wert }\end{array}$ & $\begin{array}{l}\text { Standard- } \\
\text { abweich. }\end{array}$ & $\begin{array}{c}\text { Mittel- } \\
\text { wert }\end{array}$ & $\begin{array}{l}\text { Standard- } \\
\text { abweich. }\end{array}$ & $\begin{array}{c}\text { Mittel- } \\
\text { wert }\end{array}$ & $\begin{array}{l}\text { Standard- } \\
\text { abweich. }\end{array}$ \\
\hline \multicolumn{7}{|c|}{ Priddispon. Variablen } \\
\hline Alter & 39,641 & 11,14 & 39,811 & 11,19 & 39,453 & 11,084 \\
\hline Alter ${ }^{2}$ & 1695,446 & 899,179 & 1710,098 & 911,223 & 1679,354 & 885,685 \\
\hline Geschlecht & 0,477 & 0,5 & - & - & - & 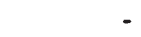 \\
\hline Interaktion & 18,803 & 21,14 & - & - & - & - \\
\hline Realschule & 0,361 & 0,48 & 0,328 & 0,469 & 0,397 & 0,489 \\
\hline FH-Reife & 0,031 & 0,172 & 0,035 & 0,185 & 0,026 & 0,158 \\
\hline Abitur & 0,086 & 0,281 & 0,076 & 0,265 & 0,097 & 0,296 \\
\hline Diplom & 0,194 & 0,396 & 0,196 & 0,397 & 0,192 & 0,394 \\
\hline Ausländer & 0,105 & 0,307 & 0,118 & 0,322 & 0,092 & 0,289 \\
\hline Behinderung & 0,064 & 0,245 & 0,069 & 0,254 & 0,058 & 0,234 \\
\hline \multicolumn{7}{|c|}{ Soziobk. Variablen } \\
\hline Partner_HH & 0,737 & 0,440 & 0,734 & 0,443 & 0,742 & 0,437 \\
\hline Kind & 0,392 & 0,488 & 0,411 & 0,492 & 0,370 & 0,483 \\
\hline Ln_HH-Netto & 7,900 & 0,454 & 7,919 & 0,436 & 7,880 & 0,471 \\
\hline Eink.-Sorgen & 0,261 & 0,439 & 0,245 & 0,43 & 0,280 & 0,449 \\
\hline PKV & 0,091 & 0,287 & 0,111 & 0,314 & 0,068 & 0,252 \\
\hline PKV_Zusatz & 0,112 & 0,315 & 0,098 & 0,298 & 0,127 & 0,333 \\
\hline \multicolumn{7}{|c|}{ Gesundheitsmerkmale } \\
\hline Gesundverhalt. & 1,419 & 0,890 & 1,369 & 0,891 & 1,475 & 0,885 \\
\hline Gesundzustand & 3,572 & 0,832 & 3,597 & 0,819 & 3,545 & 0,846 \\
\hline Anstrengung & 0,050 & 0,219 & 0,046 & 0,21 & 0,055 & 0,228 \\
\hline Arztbesuche & 1,855 & 3,037 & 1,545 & 3,032 & 2,195 & 3,006 \\
\hline $\mathrm{KH}(\mathrm{Vj})$. & 0,088 & 0,283 & 0,08 & 0,272 & 0,096 & 0,294 \\
\hline $\mathrm{AU}(\mathrm{Vj})$. & 8,419 & 23,292 & 7,9 & 20,887 & 8,990 & 25,666 \\
\hline Ln_BMI & 3,220 & 0,162 & 3,255 & 0,143 & 3,182 & 0,173 \\
\hline$\left(\mathrm{Ln}_{-}{ }_{-} \mathrm{BMI}\right)^{2}$ & 10,394 & 1,056 & 10,614 & 0,938 & 10,152 & 1,123 \\
\hline Normalgew. & 0,503 & 0,500 & 0,494 & 0,500 & 0,513 & 0,500 \\
\hline Sport & 0,291 & 0,454 & 0,277 & 0,447 & 0,306 & 0,461 \\
\hline Rauchen & 0,374 & 0,484 & 0,402 & 0,49 & 0,344 & 0,475 \\
\hline \multicolumn{7}{|c|}{ Arbeitsmerkmale } \\
\hline Arbeitszeit & 35,626 & 14,399 & 39,958 & 12,954 & 30,869 & 14,408 \\
\hline Arbeitszeit ${ }^{2}$ & 1476,488 & 860,179 & 1764,327 & 819,869 & 1160,384 & 789,694 \\
\hline
\end{tabular}

Die unterschiedliche Einkommenssituation spiegelt sich auch in dem Versichertenstatus wieder. Insgesamt sind 9,1 Prozent der Personen in der privaten Krankenversicherung versichert, und 11,2 Prozent haben eine private Zusatzversicherung. Nach Geschlechtern getrennt zeigt sich jedoch ein großer Unterschied, denn 11,1 Prozent der Männer sind Mitglied einer PKV, aber nur 6,8 Prozent der Frauen. Somit scheint auch der Bedarf an privaten Zusatzversiche- 
rungen, die in der GKV nicht enthaltene Leistungen abdecken, bei den Frauen größer zu sein als bei Männern.

Von besonderem Interesse sind mögliche Unterschiede hinsichtlich der Gesundheitsmerkmale. Der Indikator für das Gesundheitsverhalten weist in der Gesamtstichprobe einen Durchschnitt von 1,42 auf. Bei der getrennten Betrachtung zeigt sich, dass sich das Gesundheitsverhalten von Frauen und Männern nur wenig unterscheidet, denn der Mittelwert liegt mit 1,48 bei den Frauen nur etwas über dem Wert von 1,37, der in der Männerstichprobe ermittelt wurde. Anhand der deskriptiven Statistik zeigt sich weiterhin, dass Frauen in allen drei Indikatorkategorien ein gesünderes Verhalten aufweisen. Während die Verteilung des Normalgewichts mit 51,3\% der Frauen der Verteilung in der Männerstichprobe (hier sind 49,4\% normalgewichtig) noch relativ nah kommt, ist die Differenz beim Sport mit 2,9 Prozentpunkten schon etwas deutlicher. Allerdings ist auch die Streuung des BMI bei den Frauen insgesamt größer. Der deutlichste Unterschied im Verhalten ist in Bezug auf das Rauchen festzustellen, da immerhin 40,2\% der Männer angeben, regelmäßig zu rauchen, aber nur 34,4\% der Frauen.

Tabelle 6 listet die relativen Häufigkeiten der einzelnen Gesundheitskategorien, aus denen sich die Variable Gesundverhalten zusammensetzt, noch einmal nach ihren Indikatoren auf.

Tabelle 6: Verteilung der 3 Gesundheitskategorien (in \%)

\begin{tabular}{|c|l|c|c|c|c|}
\cline { 3 - 6 } \multicolumn{1}{c|}{} & \multicolumn{2}{c|}{ nicht normalgewichtig } & \multicolumn{2}{c|}{ normalgewichtig } \\
\cline { 2 - 6 } \multicolumn{1}{c|}{ in $\%$} & Raucher & $\begin{array}{c}\text { Nicht- } \\
\text { raucher }\end{array}$ & Raucher & $\begin{array}{c}\text { Nicht- } \\
\text { raucher }\end{array}$ \\
\hline \multirow{2}{*}{ Männer } & Kein Sport & 16,4 & $\underline{22,49}$ & $\underline{15,68}$ & $\underline{\underline{17,76}}$ \\
\cline { 2 - 6 } & Sport & $\underline{3,85}$ & $\underline{\underline{7,9}}$ & $\underline{\underline{4,25}}$ & $\underline{\underline{11,67}}$ \\
\hline \multirow{2}{*}{ Frauen } & Kein Sport & 13,87 & $\underline{21,09}$ & $\underline{12,86}$ & $\underline{\underline{21,58}}$ \\
\cline { 2 - 6 } & Sport & $\underline{\mathbf{3 , 6 1}}$ & $\underline{\underline{10,13}}$ & $\underline{\underline{4,05}}$ & $\underline{\underline{12,81}}$ \\
\hline
\end{tabular}

Kategorie 0: kein Kriterium ist erfüllt.

Kategorie 1: nur 1 Kriterium ist erfüllt.

Kategorie 2: 2 Kriterien sind erfüllt.

Kategorie 3: alle 3 Kriterien sind erfüllt.

Beide Geschlechter geben bezüglich der zweiten Kategorie (2 Kriterien sind erfüllt) am häufigsten an, einzig kein Sport zu treiben und sich sonst gleicherma- 
Ben gesund zu verhalten. In diese Kategorie sind mit 21,58\% zudem die meisten Frauen einzuordnen. Weiterhin zeigt sich, dass Personen, die regelmäßig Sport treiben, zumeist Nichtraucher sind, und zwar unabhängig von der Gewichtssituation.

Die meisten Männer $(22,4 \%)$ lassen sich in ein Verhaltensmuster der Kategorie 1 einordnen, indem sie zwar nicht rauchen, sich aber nicht sportlich betätigen und entsprechend nicht normalgewichtig sind. Abschließend ist zudem ein Unterschied in den Extremkategorien 0 und 3 festzustellen. Während der Unterschied derjenigen, die sich vollkommen gesund verhalten, mit $12,81 \% \mathrm{zu}$ $11,67 \%$ nur relativ knapp zugunsten der Frauenstichprobe ausfällt, zeigt sich im absolut ungesunden Verhalten eine relativ deutliche Ungleichheit, indem 16,4\% der Männer entsprechend eingeordnet werden können, aber nur 13,87\% der Frauen. Die nachfolgende Tabelle liefert noch einmal eine Aufstellung darüber, wie viel Prozent der Männer bzw. der Frauen in die jeweiligen Kategorien einzuordnen sind:

Tabelle 7: Anteil der Personen je Kategorie (Querschnitt)

\begin{tabular}{|c|c|c|}
\hline \multirow{2}{*}{ Kategorie } & Männer & Anteil der Personen \\
\hline 0 & $16,4 \%$ & $13,87 \%$ \\
\hline 1 & $42,02 \%$ & $37,56 \%$ \\
\hline 2 & $29,91 \%$ & $35,76 \%$ \\
\hline 3 & $11,67 \%$ & $12,81 \%$ \\
\hline
\end{tabular}

Obwohl Frauen gemessen an diesen Indikatoren das bessere Gesundheitsverhalten aufweisen, wird der selbst eingeschätzte Gesundheitszustand knapp niedriger bewertet als in der Stichprobe der Männer. Insgesamt schätzen gut 60 Prozent der Männer ihre Gesundheit als sehr gut oder gut ein, während nur knapp $58 \%$ der Frauen eine entsprechende Bewertung abgeben. Dieser Zusammenhang zeigt sich ebenfalls in der höheren Zahl der Arztbesuche und der Krankenhausaufenthalte. Frauen berichten häufiger über körperliche Einschränkungen bei anstrengenden Tätigkeiten. Zudem waren sie im Jahr 2003 durchschnittlich fast 9 Tage krank, während diese Zahl bei den Männern mit 7,9 Tagen deutlich darunter liegt.

\subsubsection{Ergebnisse}

Insgesamt werden nachfolgend 6 verschiedene Schätzungen präsentiert. Da es sich bei den abhängigen Variablen Gesundverhalten und Gesundzustand um or- 
dinal skalierte Variablen handelt, wird jeweils das Ordered Probit-Verfahren angewendet. Während die Varianz in einem OLS-Modell geschätzt werden kann, indem es sich bei den Werten von $y$ um tatsächliche Beobachtungen handelt, muss für latente Variablen eine Varianz für die Fehlerterme angenommen werden (vgl. Long und Freese (2003), S. 112). Eine mögliche Fehlspezifikation, die sich aus der unterstellten Verteilung der Störterme ergeben kann, wird durch die Verwendung eines robusten Schätzers umgangen (vgl. Long und Freese (2003), S. 74).

\section{Gesundheitsrelevantes Verhalten}

In der ersten Schätzung werden Einflussfaktoren des gesundheitsrelevanten Verhaltens unter Berücksichtigung der gesamten Stichprobe untersucht. Die zweite und dritte Schätzung liefern die entsprechenden Schätzergebnisse nur für Männer bzw. Frauen. Die Ergebnisse aller drei Schätzungen sind in Tabelle 8 zusammengefasst.

In der Gesamtstichprobe zeigt sich für das Alter ein signifikant negativer $\mathrm{Zu}$ sammenhang, der allerdings mit zunehmendem Alter abnimmt. Demnach sinkt die Bereitschaft, in die Gesundheit zu investieren, mit steigendem Alter. Bei der getrennten Betrachtung der Schätzungen zwei und drei zeigt sich, dass der Einfluss des Alters allein für die Männer existiert, während bei den Frauen keine Effekte festgestellt werden können. Entgegen der deskriptiven Statistik weisen Frauen zudem kein besseres Investitionsverhalten auf, vielmehr ist das geschätzte Vorzeichen sogar negativ, wenn auch eindeutig nicht signifikant. Der Interaktionsterm ist ebenfalls nicht signifikant, so dass keine geschlechtsspezifischen Alterseffekte vorhanden sind. Um mögliche Unterschiede in der Bedeutung einzelner Einflussfaktoren zu berücksichtigen, wird die Gleichung noch einmal nach Geschlecht getrennt geschätzt.

Für die Bildungsvariablen lassen sich jeweils folgende Ergebnisse feststellen: Sämtliche Bildungsindikatoren weisen ein positives Vorzeichen auf, und nahezu alle sind auf dem $1 \%$-Niveau signifikant ${ }^{159}$, so dass in der Referenzgruppe derjenigen, die maximal einen Hauptschulabschluss aufweisen können, die Wahrscheinlichkeit für ungesundes Verhalten am größten ist. Auch zeigt sich anhand der Koeffizienten über alle drei Stichproben, dass sich höhere Bildungsabschlüsse gegenüber dem Realschulabschluss ebenfalls positiv auf die Investi-

\footnotetext{
${ }^{159}$ Die Signifikanzniveaus für den Einfluss des Realschulabschlusses sind in den geschlechtsspezifischen Schätzungen mit 1,1\% bzw. 1,6\% nur knapp über dem 1\%-Niveau, so dass allgemein von einer hohen Bedeutung der Bildung für das gesundheitsrelevante Verhalten ausgegangen werden kann.
} 
tionsbereitschaft auswirken. Bezüglich der drei Variablen Fachhochschulreife, Abitur und Diplom ist allerdings kein eindeutiger Gradient festzustellen. Die Fachhochschulreife weist jeweils den höchsten Koeffizienten auf, während dem Abitur jeweils der geringste Einfluss zukommt. Eine fundierte Interpretation dieses Ergebnisses kann nicht gegeben werden. Eindeutig ist jedoch, dass die Wahrscheinlichkeit gesundheitsrelevanten Verhaltens mit der Qualität der Schulbildung ansteigt.

Die Variable Ausländer ist sowohl in der Gesamtstichprobe als auch in der Stichprobe der Männer signifikant negativ, während sich eine Behinderung lediglich für die Männer negativ auswirkt, und das auch nur sehr schwach signifikant. Somit kann der postulierte negative Zusammenhang nicht einwandfrei bestätigt werden.

Im Hinblick auf das familiäre Umfeld ergeben sich geschlechtsspezifische Unterschiede. In der Gesamtstichprobe spielt die Familienzugehörigkeit keine Rolle. Für das gesundheitsrelevante Verhalten der Männer ist das Zusammenleben mit einer Partnerin hingegen eher hinderlich, während für Frauen kein Einfluss nachweisbar ist. Deutliche Unterschiede lassen sich zudem bei der Variablen Kind beobachten. In der Stichprobe, die lediglich die Männer umfasst, ist der Einfluss eines Kindes unter 16 Jahren positiv, wenn auch nur schwach signifikant. Bei Frauen ist demgegenüber ein auf dem 1\%-Niveau signifikanter negativer Zusammenhang festzustellen. Eine mögliche Begründung für diese Unterschiede liegt in den Zuständigkeiten für die Kindererziehung, da diese nach wie vor häufig den Frauen obliegt. ${ }^{160}$ Dazu kommt, dass bei etwa einem Drittel der berufstätigen Frauen mindestens ein Kind im Alter von unter 16 Jahren im Haushalt lebt, so dass hier Kind und Beruf miteinander verknüpft werden müssen. Damit dürfte für Investitionen in die eigene Gesundheit häufig nicht ausreichend Zeit bleiben.

Gemäß den theoretischen Überlegungen geht ein steigendes Einkommen mit einer steigenden Nachfrage nach Gesundheitsleistungen und damit mit einer höheren Wahrscheinlichkeit gesundheitsrelevanten Verhaltens einher, so dass für die Einkommensvariable ein positives sowie für Einkommenssorgen ein negatives Vorzeichen postuliert wird. Die Ergebnisse der Schätzungen bestätigen diese Hypothese jeweils auf dem 1\%-Niveau. Je höher das Einkommen ist, desto wahrscheinlicher ist es also, dass alle drei Verhaltenskategorien (Nichtraucher, regelmäßig Sport und Normalgewicht) erfüllt sind, während sich Sorgen ein-

${ }^{160}$ Bundesweit sind nur 0,2\% der Văter allein in Elternzeit, und zumindest 4,7\% teilen sich die Elternzeit mit der Partnerin (vgl. Bundesministerium für Familie, Senioren, Frauen und Jugend (2004), S. 16). 
deutig negativ auswirken. Somit stellt das Einkommen neben der Bildung in dieser Querschnittsschätzung den zweiten bedeutenden Einflussfaktor dar.

Tabelle 8: Ergebnisse der Querschnittsschätzung: gesundheitsrelevantes Verhalten

Abhängige Variable: gesundheitsrelevantes Verhalten ${ }^{161}$

\begin{tabular}{|c|c|c|c|c|c|c|}
\hline & \multicolumn{2}{|c|}{ (1) ges. Stichprobe } & \multicolumn{2}{|c|}{ (2) nur Männer } & \multicolumn{2}{|c|}{ (3) nur Frauen } \\
\hline & Koeff. & P-Wert & Koeff. & P-Wert & Koeff. & P-Wert \\
\hline \multicolumn{7}{|c|}{ Prildispon. Variablen } \\
\hline Alter & $-0,0346$ & $0,001 * * *$ & $-0,0468$ & $0,001 * * *$ & $-0,0105$ & 0,501 \\
\hline Alter ${ }^{2}$ & 0,0004 & $0,002 * * *$ & 0,0006 & $0,001 * * *$ & 0,0001 & 0,499 \\
\hline Geschlecht & $-0,0996$ & 0,401 & & & & \\
\hline Interaktion & 0,0043 & 0,133 & & & & \\
\hline Realschule & 0,1452 & $0,000 * * *$ & 0,138 & $0,011 * *$ & 0,1435 & $0,016^{* *}$ \\
\hline FH-Reife & 0,4812 & $0,000 * * *$ & 0,5115 & $0,000 * * *$ & 0,4331 & $0,003 * * *$ \\
\hline Abitur & 0,3532 & $0,000 * * *$ & 0,459 & $0,000 * * *$ & 0,2635 & $0,006 * * *$ \\
\hline Diplom & 0,4058 & $0,000 * * *$ & 0,4887 & $0,000 * * *$ & 0,315 & $0,000 * * *$ \\
\hline Ausländer & $-0,0989$ & $0,055^{*}$ & $-0,148$ & $0,032 * *$ & $-0,0524$ & 0,505 \\
\hline Behinderung & $-0,101$ & 0,124 & $-0,159$ & $0,095^{*}$ & $-0,0449$ & 0,619 \\
\hline \multicolumn{7}{|c|}{ Soziobk. Variablen } \\
\hline Partner_HH & $-0,0306$ & 0,486 & $-0,1464$ & $0,021 * *$ & $-0,042$ & 0,503 \\
\hline Kind & $-0,0400$ & 0,279 & 0,0855 & $0,094^{*}$ & $-0,1721$ & $0,003 * * *$ \\
\hline Ln_HH-Netto & 0,2679 & $0,000 * * *$ & 0,3021 & $0,000 * * *$ & 0,2117 & $0,000^{* * *}$ \\
\hline Eink.-Sorgen & $-0,2394$ & $0,000 * * *$ & $-0,1508$ & $0,004^{* * *}$ & $-0,3186$ & $0,000 * * *$ \\
\hline PKV & 0,1664 & $0,004 * * *$ & 0,1826 & $0,013^{* *}$ & 0,1263 & 0,185 \\
\hline PKV_Zusatz & 0,0167 & 0,750 & $-0,0517$ & 0,503 & 0,0619 & 0,391 \\
\hline \multicolumn{7}{|c|}{ Gesundheitsmerkmale } \\
\hline Anstrengung & $-0,0375$ & 0,612 & 0,0432 & 0,703 & $-0,0905$ & 0,352 \\
\hline Arztbesuche & 0,0143 & $0,011^{* *}$ & 0,0182 & $0,030^{* *}$ & 0,0088 & 0,234 \\
\hline $\mathrm{KH}(\mathrm{Vj})$. & $-0,1267$ & $0,033^{* *}$ & $-0,0662$ & 0,468 & $-0,1712$ & $0,030 * *$ \\
\hline$A U(V j)$. & $-0,002$ & $0,008 * * *$ & $-0,0025$ & $0,046^{* *}$ & $-0,0018$ & $0,054^{*}$ \\
\hline \multicolumn{7}{|c|}{ Arbeitsmerkmale } \\
\hline Arbeitszeit & 0,0113 & $0,001 * * *$ & 0,0119 & $0,010^{* * *}$ & 0,0128 & $0,031 * *$ \\
\hline Arbeitszeit ${ }^{2}$ & $-0,0003$ & $0,000 * * *$ & $-0,0003$ & $0,000 * * *$ & $-0,0003$ & $0,002 * * *$ \\
\hline $\begin{array}{l}\text { Log } \\
\text { Likelihood }\end{array}$ & $-5877,45$ & & $-3049,6$ & & $-2807,73$ & \\
\hline Wald $\mathrm{Chi}^{2}$ & 397,25 & $0,000 * * *$ & 245,69 & $0,000 * * *$ & 164,53 & $0,000 * * *$ \\
\hline $\begin{array}{l}\text { McFadden's } \\
\mathrm{R}^{2}\end{array}$ & 0,033 & & 0,039 & & 0,029 & \\
\hline 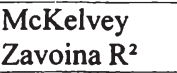 & 0,091 & & 0,108 & & 0,080 & \\
\hline AIC & 2,477 & & 2,464 & & 2,493 & \\
\hline Beobacht. & 4765 & & 2494 & & 2271 & \\
\hline
\end{tabular}

$161 *$ signifikant auf dem 10\%-Niveau, ** signifikant auf dem 5\%-Niveau, *** signifikant auf dem $1 \%$-Niveau. 
Im Hinblick auf den Versichertenstatus zeigen sich erneut Unterschiede zwischen Männern und Frauen. In der Schätzung für die Gesamtstichprobe weisen privat versicherte Personen ein größeres Gesundheitsbewusstsein auf. Dieser Zusammenhang bestätigt sich für die Männer ebenfalls deutlich, aber nicht für die Frauen. Eine mögliche Begründung für den positiven Einfluss ist ein positiver Selbstbeteiligungssatz, so dass generell das Interesse an einer guten Gesundheit größer ist, da nicht alle medizinischen Leistungen vollständig durch die Versicherung übernommen werden. Darüber hinaus haben Frauen statistisch gesehen ein höheres Erkrankungsrisiko ${ }^{162}$ sowie eine höhere Lebenserwartung, so dass privat versicherte Frauen c. p. höhere Prämien zahlen als Männer (vgl. Wasem und Greß (2004)). Diese höhere finanzielle Belastung könnte mit einem geringeren Anreiz zum gesundheitsrelevanten Verhalten einhergehen, indem private Investitionen gegen medizinische Leistung substituiert werden. Private $\mathrm{Zu}$ satzversicherungen wirken sich für alle Stichproben nicht auf das Verhalten aus.

Die dritte untersuchte Kategorie umfasst mit den Gesundheitsmerkmalen auch die Inanspruchnahme medizinischer Leistungen. Eine Gesundheitsbeeinträchtigung bei anstrengenden Tätigkeiten wirkt sich in keiner Weise auf das Investitionsverhalten aus. Für die Zahl der Arztbesuche zeigt sich ein auf dem 5\%-Niveau signifikant positiver Effekt für die Gesamtstichprobe sowie die Teilstichprobe der Männer. Damit wird ein positives Gesundheitsverhalten umso wahrscheinlicher, je mehr Arztbesuche vorliegen. Für Frauen lässt sich hier jedoch erneut kein eindeutiger Einfluss nachweisen. Hingegen zeigt sich bei der Betrachtung der Krankenhausaufenthalte des Vorjahres ein entgegengesetztes Bild, denn hierdurch wird lediglich das Verhalten der Frauen negativ beeinflusst. Für alle drei Stichproben zeigt sich weiterhin ein negativer Einfluss von Arbeitsunfähigkeit aus dem Vorjahr, wodurch Zeitverzögerungseffekte abgebildet werden können.

Zuletzt bleibt die Frage nach dem einkommens- und bildungsbereinigten Einfluss der Arbeit, der allein durch die reine Arbeitszeit abgebildet wird. Für alle drei Stichproben wird der a priori postulierte konkave Zusammenhang bestätigt. Aus den geschätzten Koeffizienten lässt sich nun diejenige Arbeitszeit ermitteln, bei der die Wahrscheinlichkeit eines sehr gesunden Verhaltens maximal wird. Für die Gesamtstichprobe ergibt sich eine optimale Arbeitszeit von 20,01 Stunden, für die Gruppe der Männer liegt diese Zeit bei 21,75 Stunden und für die Frauen bei 19,21 Stunden. Geht die Arbeitszeit darüber hinaus, steigt die Wahrscheinlichkeit eines ungesunden Verhaltensmerkmals, indem beispielsweise Stress durch Tabakkonsum kompensiert wird oder neben zusätzlichen Haushaltsaufgaben nicht genügend Zeit für Sport zur Verfügung steht. Damit können

${ }^{162}$ Zumindest im Alter von 18 bis 55 Jahren. 
die Hypothesen 1 und 2 für die vorliegenden Querschnittsschätzungen bestätigt werden.

Die Wald-Statistik ${ }^{163}$ ist jeweils signifikant von Null verschieden. Damit können die Modellzusammenhänge nicht allein durch die jeweiligen Konstanten erklärt werden. Das $\mathrm{R}^{2}$ von McFadden ist bei allen drei Schätzungen mit 0,029 für die Stichprobe der Frauen und 0,039 für die der Männer relativ gering. Das $\mathrm{R}^{2}$ von McKelvey und Zavoina hingegen liegt mit 0,080 bzw. 0,108 deutlich darüber. Die Aussagekraft des Modells scheint damit für die Stichprobe der Männer gröBer zu sein als für Frauen, was sich auch in den AIC-Werten von 2,493 bzw. 2,464 niederschlägt (nähere Erläuterungen zu den verwendeten Gütemaßen befinden sich in Kapitel A.4 im Anhang).

\section{Selbst eingeschätzter Gesundheitszustand}

In der zweiten Schätzung werden die Determinanten des selbst eingeschätzten Gesundheitszustands bestimmt. Hier stellt sich die Frage, inwiefern sich Faktoren, die das gesundheitsrelevante Verhalten beeinflussen, auch auf die eigene Gesundheit auswirken, wenn die Verhaltensindikatoren eigens in die Schätzung eingehen. ${ }^{164}$ Die Ergebnisse der Schätzungen sind in Tabelle 9 aufgeführt.

Im Hinblick auf die prädisponierenden Variablen ergeben sich deutliche Unterschiede zwischen dem Verhalten auf der einen und dem Gesundheitszustand auf der anderen Seite. Für das Investitionsverhalten sind die Ausbildung sowie das Einkommen maßgeblich. Für die Einschätzung der Gesundheit per se sind die Bildungsvariablen hingegen ohne Bedeutung. Hier üben neben dem Geschlecht insbesondere das Alter sowie das Vorliegen einer Behinderung einen negativen Einfluss auf die Einschätzung aus. In Bezug auf das Alter kann zudem von einem linearen Zusammenhang ausgegangen werden, da der quadrierte Altersterm nicht signifikant von Null verschieden ist.

${ }^{163}$ Die Wald-Statistik gibt Aufschluss darüber, ob die verwendeten Variablen tatsächlich einen Erklärungsgehalt besitzen oder ob es sich vielmehr um zufällige Variationen handelt. Hierzu wird die Hypothese aufgestellt, dass alle Koeffizienten mit Ausnahme der Konstanten gleich Null sind. Je weiter die geschätzten Werte von dieser Restriktion abweichen, desto eher kann diese Hypothese verworfen werden (vgl. Long (1997), S. 90).

${ }^{164}$ An dieser Stelle werden die Verhaltensindikatoren separat als erklärende Variablen in die Schätzung aufgenommen, um mögliche Unterschiede in den Auswirkungen auf die Gesundheitseinschätzung zu berücksichtigen. Eine Verwendung des Index würde in diesem Fall weniger Aufschluss über die Relation zwischen dem Verhalten einerseits und der Gesundheit per se andererseits geben. 
Tabelle 9: Ergebnisse der Querschnittsschätzung: eingeschätzter Gesundheitszustand

Abhăngige Variable: Selbst eingeschätzter Gesundheitszustand

\begin{tabular}{|c|c|c|c|c|c|c|}
\hline & \multicolumn{2}{|c|}{ (1) ges. Stichprobe } & \multicolumn{2}{|c|}{ (2) nur Männer } & \multicolumn{2}{|c|}{ (3) nur Frauen } \\
\hline & Koeff. & P-Wert & Koeff. & P-Wert & Koeff. & P-Wert \\
\hline \multicolumn{7}{|c|}{ Pridispon. Variablen } \\
\hline Alter & $-0,0311$ & $0,005 * * *$ & $-0,0434$ & $0,004 * * *$ & $-0,0145$ & 0,376 \\
\hline Alter ${ }^{2}$ & 0,0001 & 0,682 & 0,0002 & 0,356 & 0,0001 & 0,737 \\
\hline Geschlecht & $-0,2303$ & $0,067^{*}$ & & & & \\
\hline Interaktion & 0,0045 & 0,126 & & & & \\
\hline Realschule & $-0,0388$ & 0,340 & $-0,0669$ & 0,231 & $-0,0135$ & 0,822 \\
\hline FH-Reife & $-0,1410$ & 0,149 & $-0,0770$ & 0,147 & $-0,0814$ & 0,579 \\
\hline Abitur & 0,0161 & 0,811 & $-0,0770$ & 0,418 & 0,0943 & 0,327 \\
\hline Diplom & 0,0007 & 0,989 & $-0,0559$ & 0,420 & 0,0584 & 0,430 \\
\hline Ausländer & 0,1347 & $0,021 * *$ & 0,2215 & $0,003 * * *$ & 0,0062 & 0,947 \\
\hline Behinderung & $-0,3840$ & $0,000 * * *$ & $-0,3368$ & $0,001 * * *$ & $-0,4558$ & $0,000 * * *$ \\
\hline \multicolumn{7}{|c|}{ Soziozk. Variablen } \\
\hline Partner_HH & $-0,0771$ & 0,085 & $-0,0655$ & 0,666 & $-0,1260$ & $0,047 * *$ \\
\hline Kind & 0,0516 & 0,174 & 0,0475 & 0,371 & 0,0133 & 0,822 \\
\hline Ln_HH-Netto & 0,0483 & 0,242 & 0,0607 & 0,303 & 0,0418 & 0,478 \\
\hline Eink.-Sorgen & $-0,2661$ & $0,000 * * *$ & $-0,3473$ & $0,000 * * *$ & $-0,2001$ & $0,000 * * *$ \\
\hline PKV & $-0,0481$ & 0,444 & 0,0104 & 0,896 & $-0,1613$ & 0,120 \\
\hline PKV_Zusatz & $-0,0028$ & 0,957 & $-0,0959$ & 0,206 & 0,0768 & 0,259 \\
\hline \multicolumn{7}{|c|}{ Gesundheitsmerkmale } \\
\hline Anstrengung & $-1,0509$ & $0,000 * * *$ & $-0,8605$ & $0,000 * * *$ & $-1,2312$ & $0,000 * * *$ \\
\hline Ln_BMI & 8,8165 & $0,003 * * *$ & 9,3352 & $0,025^{*}$ & 8,6445 & $0,044^{* *}$ \\
\hline$\left(\mathrm{Ln}_{-} \_\mathrm{BMI}\right)^{2}$ & $-1,4455$ & $0,001 * * *$ & $-1,5165$ & $0,016^{* *}$ & $-1,4280$ & $0,032 * *$ \\
\hline Rauchen & $-0,1803$ & $0,000 * * *$ & $-0,1841$ & $0,000 * * *$ & $-0,1836$ & $0,000 * * *$ \\
\hline Sport & 0,2347 & $0,000 * * *$ & 0,3074 & $0,000^{* * *}$ & 0,1540 & $0,003^{* * *}$ \\
\hline Arztbesuche & $-0,0898$ & $0,000 * * *$ & $-0,0855$ & $0,000 * * *$ & $-0,0950$ & $0,000 * * *$ \\
\hline $\mathrm{KH}(\mathrm{Vj})$. & $-0,2159$ & $0,001 * * *$ & $-0,2283$ & $0,026 * *$ & $-0,2017$ & $0,024^{* *}$ \\
\hline$A U(V j)$. & $-0,0050$ & $0,000 * * *$ & $-0,0052$ & $0,001 * * *$ & $-0,0046$ & $0,000 * * *$ \\
\hline \multicolumn{7}{|c|}{ Arbeitsmerkmale } \\
\hline Arbeitszeit & 0,0069 & 0,061 & 0,0101 & $0,043 * *$ & 0,0040 & 0,501 \\
\hline Arbeitszeit ${ }^{2}$ & $-0,0001$ & $0,046 * *$ & $-0,0001$ & $0,064^{*}$ & $-0,0001$ & 0,320 \\
\hline $\begin{array}{l}\text { Log } \\
\text { Likelihood }\end{array}$ & $-4978,68$ & & $-2553,49$ & & $-2406,26$ & \\
\hline Wald $\mathrm{Chi}^{2}$ & 1107,62 & $0,000 * * *$ & 628,95 & $0,000 * * *$ & 515,84 & $0,000 * * *$ \\
\hline $\begin{array}{l}\text { McFadden's } \\
\mathrm{R}^{2}\end{array}$ & 0,134 & & 0,140 & & 0,133 & \\
\hline $\begin{array}{l}\text { McKelvey } \\
\text { Zavoina } \mathrm{R}^{2}\end{array}$ & 0,324 & & 0,333 & & 0,325 & \\
\hline AIC & 2,102 & & 2,070 & & 2,144 & \\
\hline Beobacht. & 4765 & & 2494 & & 2271 & \\
\hline
\end{tabular}

Bei der nach Männern und Frauen getrennten Beobachtung der Ergebnisse zeigt sich weiterhin, dass der Alterseffekt für die Gruppe der Männer hoch signifikant 
ist, während die Hypothese, dass das Alter bei der Gruppe der Frauen eine Rolle spielt, wieder verworfen werden kann. Darüber hinaus schätzen männliche Ausländer ihre Gesundheit c. p. besser ein als deutsche Männer, obwohl sie signifikant weniger in ihre Gesundheit investieren. Dieser Effekt kann für die Frauen nicht nachgewiesen werden.

Für die sozioökonomischen Variablen gilt, dass hier nur noch die Einkommenssorgen über alle drei Stichproben hoch signifikant sind, während die übrigen Variablen jeweils keinen Einfluss ausüben. Damit spielt das tatsächlich verfügbare Einkommen für die Gesundheit per se keine Rolle, obwohl diese im Hinblick auf die Gesundheitsinvestitionen einen maßgeblichen Einflussfaktor darstellen (vgl. Tabelle 8). Dieser Zusammenhang bestätigt die grundsätzlich einkommensunabhängige Ausgestaltung des deutschen Gesundheitssystems, in dem der Zugang zu Gesundheitsleistungen unabhängig vom Einkommen gewährt wird. Nichtsdestotrotz wirken sich wirtschaftliche Sorgen negativ auf die Gesundheit aus, weshalb davon ausgegangen werden muss, dass nicht das absolute Einkommen, sondern die zum Bedarf relative Einkommensposition von besonderer Bedeutung ist.

In der Liste der Gesundheitsmerkmale sind neben den in der ersten Schätzreihe verwendeten Variablen zusätzlich noch die Verhaltensindikatoren Gewicht, Rauchen und Sport enthalten. In der ersten Schätzung, die die gesamte Stichprobe umfasst, sind alle Indikatoren auf dem 1\%-Niveau signifikant, und auch bei den nach dem Geschlecht getrennten Stichproben findet sich ein ähnliches Ergebnis. Der Einfluss des logarithmierten BMI ist jeweils positiv, wenn auch mit abnehmender Rate. Folglich sind weder Unter- noch Übergewicht der Gesundheit zuträglich. Für die Männer ergibt sich aus den Koeffizienten ein gesundheitsoptimaler BMI von 21,71, der Optimalwert der Frauen liegt bei 20,63. Für beide Geschlechter liegt dieser BMI im Bereich des altersunabhängigen Normalgewichts gemäß der WHO-Definition. Es kann daher davon ausgegangen werden, dass die Wahrscheinlichkeit einer sehr guten Gesundheit für normalgewichtige Personen am größten ist. Darüber hinaus wirkt sich das Rauchen eindeutig negativ auf die Gesundheit aus, und auch der postulierte positive Einfluss sportlicher Betätigung kann bestätigt werden.

Die Zahl der Arztbesuche im letzten Quartal beeinflusst die Wahrscheinlichkeit einer guten Gesundheit ebenso negativ wie mögliche Krankenhausaufenthalte sowie Arbeitsunfähigkeit im Vorjahr. Hierin zeigt sich, dass Gesundheitsprobleme in der Regel langfristiger Natur sind und sich daher bis in nachfolgende Perioden auswirken. 
Der konkave Zusammenhang für die Arbeitszeit, der sich für beide Geschlechter in der Verhaltensschätzung finden lässt, besitzt in der Zustandsschätzung nur noch für die Stichprobe der Männer sowie die Gesamtstichprobe Gültigkeit. Die für Männer gesundheitsoptimale Arbeitszeit liegt demnach bei 35,57 Stunden. Damit ist die Bedeutung der Arbeitszeit für das gesundheitsrelevante Verhalten sowie für die Gesundheit per se sehr unterschiedlich. Während die reine Arbeitszeit die für Ernährung und Sport verfügbare Zeit determiniert, sind diese Verhaltensindikatoren in der Zustandsschätzung enthalten, so dass hier letztlich ein reiner Zeiteffekt bestimmt wird, bei dem positive Einflüsse der Arbeit wie Motivation den negativen Einflüssen, beispielsweise Stress oder körperliche Anstrengung, entgegenwirken. Für die Gesundheitseinschätzung von Frauen ist die Arbeitszeit hingegen nicht von Bedeutung. In der Regel üben Frauen seltener körperlich anstrengende Tätigkeiten aus als Männer. Darüber hinaus ist die durchschnittliche Arbeitszeit mit knapp 31 Stunden gegenüber der Arbeitszeit von Männern mit knapp 40 Stunden wesentlich niedriger, so dass hier davon ausgegangen werden kann, dass auch die Belastung durch die Erwerbstätigkeit entsprechend geringer ist.

Die Wald-Statistik ist für alle drei Stichproben erneut signifikant von Null verschieden. Das $\mathrm{R}^{2}$ von McFadden liegt bei diesen Schätzungen zwischen 0,133 und 0,14 und ist damit deutlich größer als in der Verhaltensschätzung. Gleiches gilt für das $\mathrm{R}^{2}$ von McKelvey und Zavoina, das zwischen 0,323 und 0,332 liegt. Erneut scheint der Erklärungsgehalt des Modells für die Männer am größten zu sein, da auch der AIC-Wert mit 2,07 unter dem Wert bei den Frauen $(2,145)$ liegt.

\subsection{Panelanalyse}

\subsubsection{Vorteile und Probleme der Panelschätzung}

Die Panelschätzung vereint die Vorteile von Quer- und Längsschnittanalysen, indem Haushalte oder einzelne Individuen über mehrere Perioden hinweg betrachtet werden. Auf diese Weise erhält man mehr Beobachtungen, die überdies Veränderungen in der Zeit abbilden. Hieraus ergeben sich folgende Vorteile (vgl. Kennedy (2003), S. $301 \mathrm{f}$ ):

Erstens fuhhren Unterschiede im Verhalten von Individuen, die nicht oder nur unter Verwendung zahlreicher weiterer erklärenden Variablen gemessen werden können, ebenso zu Verzerrungen wie fehlende Zeitreihenvariablen, die das Verhalten in jeder Periode unterschiedlich beeinflussen. Durch die Verwendung von Paneldaten kann dieser Heterogenität Rechnung getragen werden. Zweitens erlauben Paneldaten mehr Variation in den Variablen, wodurch die Wahrscheinlichkeit von Multikollinearität verringert wird. Es stehen insgesamt mehr Infor- 
mationen über die einzelnen erklärenden Variablen zur Verfügung. Drittens bieten Paneldaten schließlich eine bessere Anpassungsdynamik als Querschnittsdaten, in denen Veränderungen in der Zeit nicht enthalten sind. In einer reinen Längsschnittbetrachtung ist die Dynamik nur dann beobachtbar, wenn die Zeitreihe hinreichend lang ist, da nur dann ausreichend Beobachtungen zur Verfügung stehen. Eine entsprechend lange Periode ist im Panel nicht notwendig, da hier die Dynamik über viele Individuen betrachtet wird, so dass in der Regel wenige Zeitpunkte ausreichen. ${ }^{165}$

Ein wesentliches Problem stellt hingegen die Erfassung der Daten dar (vgl. Baltagi (2005), S. 7-9). Insbesondere bei der Beantwortung von Fragen über mehrere Perioden kann es zu widersprüchlichen Aussagen durch die befragten Personen kommen, sei es durch eine unklare Fragestellung oder durch Erinnerungslücken. In einem Querschnitt sind derartige Messfehler nicht als solche zu identifizieren, im Panel führen sie zu Inkonsistenzen, so dass Korrekturen notwendig sind. Aus diesem Grund sind im SOEP generierte Variablen enthalten, die entsprechende Fehler ausgleichen, indem Daten vorangegangener Perioden ebenfalls berücksichtigt werden.

Ebenso kann es sein, dass Individuen entweder Fragen nicht beantworten (nonresponse) oder aus beliebigen Gründen nicht weiter an der Befragung teilnehmen, was gemeinhin als Panelsterblichkeit bezeichnet wird. In diesen Fällen ist die Stichprobe nicht mehr absolut zufällig, so dass die Repräsentativität in Frage gestellt werden muss. Darüber hinaus sind kurze Zeiträume nicht geeignet, sobald es sich um Variablen mit asymptotischen Eigenschaften handelt, da der Annäherungsprozess über mehrere Perioden hinweg zu beobachten ist.

Für die nachfolgenden Schätzungen wird aufgrund der Ordinalskalierung der beiden abhängigen Variablen ein Random Effects ${ }^{166}$ Ordered Probit-Modell angewendet. Im Rahmen dieser Modellspezifikation sind die Elemente des Fehlerterms $\varepsilon_{i t}, v_{i t}$ und $u_{i}$, unabhängige Zufallsvariablen, für die folgende Annahmen gelten (vgl. Greene (2003), S. 690f.):

$$
\begin{aligned}
& E\left[v_{i t}\right]=0, \\
& \operatorname{Cov}\left[v_{i t}, v_{j s}\right]=\operatorname{Var}\left[v_{i t}\right]=1 \quad \text { für } i=j \text { und } t=s, 0 \text { sonst, }
\end{aligned}
$$

${ }^{165} \mathrm{Zu}$ weiteren Vorteilen siehe Baltagi (2005), S. $6 \mathrm{f}$.

${ }^{166} \mathrm{Zu}$ einer genauen Abgrenzung von Fixed und Random Effects siehe Kapitel B im Anhang. 


$$
\begin{aligned}
& E\left[u_{i}\right]=0 \\
& \operatorname{Cov}\left[u_{i}, u_{j}\right]=\operatorname{Var}\left[u_{i}\right]=\sigma_{u}^{2} \quad \text { für } i=j, 0 \text { sonst }, \\
& \operatorname{Cov}\left[v_{i t}, u_{j}\right]=0 \quad \text { für alle } i, t, j .
\end{aligned}
$$

Damit sind die Elemente des Fehlerterms, $v_{i t}$ und $u_{i}$, unkorreliert und jeweils unabhängig von $\boldsymbol{x}$. Weiterhin sind die Fehlerterme normalverteilt und homoskedastisch, da sie eine konstante Varianz aufweisen. Aus diesen Annahmen lassen sich für den gesamten Fehlerterm folgende Bedingungen ableiten:

$$
\begin{aligned}
& E\left[\varepsilon_{i t}\right]=0, \\
& \operatorname{Var}\left(\varepsilon_{i t}\right)=\sigma_{v}^{2}+\sigma_{u}^{2}=1+\sigma_{u}^{2}, \\
& \operatorname{Corr}\left[\varepsilon_{i t}, \varepsilon_{i s}\right]=\rho=\frac{\sigma_{u}^{2}}{1+\sigma_{u}^{2}} .
\end{aligned}
$$

wobei $\rho$ die Korrelation der kombinierten Fehlerterme der verwendeten Jahre angibt. Die Schätzung erfolgt unter Verwendung der folgenden Log-LikelihoodFunktion: ${ }^{167}$

$$
\ln L=\sum_{i=1}^{N} \ln \left(P\left(y_{i 1}, y_{i 2}, \ldots, y_{i T}\right)\right)
$$

\subsubsection{Deskriptive Statistik}

Für die nachfolgende Panelschätzung werden die Wellen 2002 und 2004 des SOEP verwendet, da diese einzig Informationen über Körpergröße und -gewicht der befragten Individuen enthalten. Da die Variable Sport lediglich Bestandteil der Welle des Jahres 2004 ist, ist diese nicht für die Panelschätzung verfügbar, während alle übrigen im Querschnitt verwendeten Variablen auch in die Panelschätzung mit eingehen. Durch die fehlende Sport-Variable kann das Gesundheitsverhalten nunmehr nur noch durch die beiden Gesundheitsindikatoren BMI

${ }^{167}$ Die genaue Modellerlăuterung zur Schätzung eines Random Effects Ordered Probit-Modells mit Paneldaten findet sich bei French (2000). 
sowie Rauchen abgebildet werden. Für die Variable Gesundverhalten ergeben sich im Panel damit folgende Ausprägungen:

0 Die Person raucht und hat ein Gewicht, das vom Normalgewicht abweicht.

1 Die Person ist entweder Nichtraucher oder normalgewichtig.

2 Die Kriterien Nichtraucher und Normalgewicht sind jeweils erfüllt.

Tabelle 10 Zeigt zudem die Aufteilung der Befragten auf die einzelnen Gesundheitskategorien, getrennt nach Frauen und Männern:

Tabelle 10: Verteilung der zwei Gesundheitskategorien (Panel)

\begin{tabular}{|l|c|c|c|}
\cline { 2 - 4 } \multicolumn{1}{c|}{} & in \% & nicht normalgewichtig & normalgewichtig \\
\hline \multirow{2}{*}{ Männer } & Raucher & 20,19 & $\underline{\mathbf{2 9 , 7 6}}$ \\
\cline { 2 - 4 } & Nichtraucher & $\underline{20,97}$ & $\underline{\mathbf{2 9 , 0 7}}$ \\
\hline \multirow{2}{*}{ Frauen } & Raucherin & 16,91 & $\underline{31,50}$ \\
\cline { 2 - 4 } & Nichtraucherin & $\underline{17,12}$ & $\underline{\mathbf{3 4 , 4 7}}$ \\
\hline Kategorie 0: kein Kriterium ist erfüllt. \\
\hline \multicolumn{2}{|l}{ Kategorie 1: nur 1 Kriterium ist erfüllt. } \\
\hline \multicolumn{2}{|l}{ Kategorie 2: beide Kriterien sind erfüllt. } \\
\hline
\end{tabular}

Die zusätzliche Berücksichtigung der Welle 2002 bringt kaum Unterschiede bei den Verhaltensindikatoren mit sich, da sowohl der Anteil der Normalgewichtigen als auch der Nichtraucher annähernd konstant geblieben sind. ${ }^{168}$ Somit sind die meisten Männer nach wie vor zwar Nichtraucher, gleichzeitig aber auch nicht normalgewichtig $(29,76 \%)$. Für die meisten Frauen sind sogar beide Kategorien zutreffend $(34,47 \%)$.

In Tabelle 11 erfolgt noch einmal eine Zusammenfassung der Kategorien:

\footnotetext{
${ }^{168}$ Auch hier würde der Anteil der Übergewichtigen ohne Altersbereinigung zunehmen und zwar insgesamt um 13,43 Prozentpunkte. Letztlich zeigt sich hier zudem, dass die Berucksichtigung des Alters insbesondere den Männern zugute kommt, da hier 16,05\% der Männer betroffen sind, während es bei den Frauen nur 10,34\% sind.
} 
Tabelle 11: Anteil der Personen je Kategorie (Panel)

\begin{tabular}{|c|c|c|}
\hline \multirow{2}{*}{ Kategorie } & Männer & Anteil der Personen \\
\hline 0 & $20,19 \%$ & $16,91 \%$ \\
\hline 1 & $50,74 \%$ & $48,62 \%$ \\
\hline 2 & $29,07 \%$ & $34,47 \%$ \\
\hline
\end{tabular}

Tabelle 12 liefert die deskriptive Statistik der Panelanalyse. Die prädisponierenden Variablen weisen mit Ausnahme der Bildungsvariablen im Vergleich mit dem Querschnittsdatensatz keine besonderen Abweichungen auf. Allerdings ist der Bildungsunterschied zugunsten der Frauen auch im Panel augenscheinlich. Die Analyse von Mittelwert und Standardabweichung des logarithmierten Haushaltsnettoeinkommens zeigt darüber hinaus, dass Frauen trotz höherer Bildungsabschlüsse weniger verdienen und dass zudem die Streuung der Einkommen hier größer ist. Diese Werte lassen sich erneut auf die Unterschiede in der Arbeitszeit zurückführen, da Männer im Durchschnitt nach wie vor eine deutlich längere Arbeitszeit aufweisen (41 zu 32 Stunden) und auch hier die Streuung wesentlich geringer ist. Zudem liegt das logarithmierte Haushaltsnettoeinkommen im Durchschnitt über den Werten aus der Querschnittsanalyse. Der Anteil derer, die sich mit Einkommenssorgen belastet sehen, beträgt in der Gesamtstichprobe des Panels etwa ein Fünftel. Diese Zahl setzt sich aus knapp 18,3\% im Jahr 2002 und bereits knapp 25\% im Jahr 2004 zusammen, wodurch die schlechte wirtschaftliche Entwicklung der vergangenen Jahre in Deutschland abgebildet wird.

Leichte Unterschiede ergeben sich auch im Hinblick auf die Gesundheitsmerkmale. Zwar geben Frauen auch im Panel an, sich häufiger gesundheitsbewusst zu verhalten, eine direkte Vergleichbarkeit der Daten ist unter Berücksichtigung der fehlenden Sport-Variablen jedoch nicht möglich. Trotz des gesünderen Verhaltens geben Frauen auch im Panel an, über eine durschnittlich schlechtere Gesundheit zu verfügen.

Zuletzt sind noch die einzelnen Werte des Gesundheitsverhaltens von Interesse. Diese unterliegen in dieser kurzen Zeitspanne kaum Veränderungen, denn nicht nur die Werte für den durchschnittlichen BMI sind nahezu konstant geblieben, sondern auch der Anteil derjenigen, die über Normalgewicht verfügen sowie derjenigen, die angeben, Raucher zu sein. Diese Zusammenhänge gelten im Übrigen für die Gesamtstichprobe ebenso wie für die nach Geschlechtern getrennte Betachtung. 
Tabelle 12: Deskriptive Statistik der Panelanalyse

\begin{tabular}{|c|c|c|c|c|c|c|}
\hline & \multicolumn{2}{|c|}{$\begin{array}{r}\text { Gesamt } \\
N=7497\end{array}$} & \multicolumn{2}{|c|}{$\begin{array}{l}\text { Männer } \\
N=4062\end{array}$} & \multicolumn{2}{|c|}{$\begin{array}{c}\text { Frauen } \\
\mathrm{N}=3435\end{array}$} \\
\hline & $\begin{array}{c}\text { Mittel- } \\
\text { wert }\end{array}$ & $\begin{array}{l}\text { Standard- } \\
\text { abweich. }\end{array}$ & $\begin{array}{c}\text { Mittel- } \\
\text { wert }\end{array}$ & $\begin{array}{l}\text { Standard- } \\
\text { abweich. }\end{array}$ & $\begin{array}{c}\text { Mittel- } \\
\text { wert }\end{array}$ & $\begin{array}{l}\text { Standard- } \\
\text { abweich. }\end{array}$ \\
\hline \multicolumn{7}{|c|}{ Pridispon. Variablen } \\
\hline Alter & 40,145 & 10,617 & 40,101 & 10,701 & 40,196 & 10,508 \\
\hline Alter ${ }^{2}$ & 1724,298 & 865,078 & 1722,772 & 878,864 & 1726,102 & 848,61 \\
\hline Geschlecht & 0,458 & 0,498 & - & - & - & - \\
\hline Interaktion & 18,417 & 21,254 & - & - & - & - \\
\hline Realschule & 0,366 & 0,482 & 0,328 & 0,47 & 0,410 & 0,492 \\
\hline FH-Reife & 0,031 & 0,172 & 0,036 & 0,187 & 0,024 & 0,152 \\
\hline Abitur & 0,079 & 0,269 & 0,074 & 0,262 & 0,084 & 0,278 \\
\hline Diplom & 0,204 & 0,403 & 0,203 & 0,402 & 0,206 & 0,405 \\
\hline Ausländer & 0,104 & 0,305 & 0,116 & 0,32 & 0,089 & 0,285 \\
\hline Behinderung & 0,061 & 0,239 & 0,062 & 0,241 & 0,06 & 0,237 \\
\hline \multicolumn{7}{|c|}{ Soziő̈k. Variablen } \\
\hline Partner_HH & 0,754 & 0,341 & 0,751 & 0,433 & 0,757 & 0,429 \\
\hline Kind & 0,398 & 0,49 & 0,428 & 0,495 & 0,363 & 0,481 \\
\hline Ln_HH-Netto & 7,915 & 0,438 & 7,932 & 0,412 & 7,894 & 0,465 \\
\hline Eink.-Sorgen & 0,216 & 0,412 & 0,205 & 0,403 & 0,23 & 0,421 \\
\hline PKV & 0,093 & 0,29 & 0,113 & 0,317 & 0,069 & 0,253 \\
\hline PKV_Zusatz & 0,11 & 0,313 & 0,096 & 0,294 & 0,127 & 0,333 \\
\hline \multicolumn{7}{|c|}{ Gesundheitsmerkmale } \\
\hline Gesundverhalt & 1,128 & 0,697 & 1,089 & 0,696 & 1,175 & 0,695 \\
\hline Gesundzustand & 3,56 & 0,807 & 3,589 & 0,788 & 3,526 & 0,828 \\
\hline Anstrengung & 0,049 & 0,215 & 0,041 & 0,199 & 0,057 & 0,233 \\
\hline Arztbesuche & 1,9 & 3,094 & 1,556 & 2,804 & 2,307 & 3,36 \\
\hline $\mathrm{KH}(\mathrm{Vj})$. & 0,083 & 0,276 & 0,078 & 0,268 & 0,089 & 0,285 \\
\hline$A U(V j)$. & 8,48 & 21,833 & 7,881 & 19,511 & 9,189 & 24,278 \\
\hline Ln_BMI & 3,223 & 0,158 & 3,255 & 0,138 & 3,184 & 0,17 \\
\hline$\left(\mathrm{Ln} \_\mathrm{BMI}\right)^{2}$ & 10,41 & 1,024 & 10,616 & 0,906 & 10,167 & 1,099 \\
\hline Normalgew. & 0,507 & 0,5 & 0,5 & 0,5 & 0,515 & 0,5 \\
\hline Rauchen & 0,379 & 0,485 & 0,412 & 0,492 & 0,34 & 0,474 \\
\hline \multicolumn{7}{|c|}{ Arbeitsmerkmale } \\
\hline Arbeitszeit & 37,112 & 12,992 & 41,036 & 11,314 & 32,472 & 13,317 \\
\hline Arbeitszeit ${ }^{2}$ & 1546,057 & 812,833 & 1811,882 & 761,127 & 1231,711 & 758,094 \\
\hline
\end{tabular}

\subsubsection{Ergebnisse}

Die nachfolgenden Ergebnisse beziehen sich auf eine Random-Effects-Schätzung für geordnete Kategorien, die aufgrund des großen Stichprobenumfangs von 7497 Beobachtungen einer Schätzung mit fixen Effekten vorzuziehen ist. Analog zur Vorgehensweise bei den Querschnittsschätzungen wird zunächst das gesundheitsrelevante Verhalten analysiert, indem sowohl die Gesamtstichprobe 
als auch jeweils nach Geschlecht getrennte Teilstichproben verwendet werden. Anschließend werden erneut die Einflussfaktoren für die Selbsteinschätzung des Gesundheitszustandes untersucht.

\section{Gesundheitsrelevantes Verhalten}

Bei der Interpretation der in Tabelle 13 aufgeführten Werte für das gesundheitsrelevante Verhalten ist maßgeblich zu berücksichtigen, dass diese Variable im Panel nur noch drei statt wie im Querschnitt vier Dimensionen aufweist. Durch das Fehlen der Variablen Sport ist ein Rückgang der Einkommens- und Arbeitszeiteffekte zu erwarten, da regelmäßiger Sport sowohl zeit- als auch einkommensintensiv ist.

Die wesentlichen Ergebnisse aus der Querschnittsschätzung lassen auch für die Panelanalyse bestätigen. Nach wie vor spielt - von der Fachhochschulreife bei Frauen einmal abgesehen - die Bildung eine zentrale Rolle für die Bereitschaft zu Gesundheitsinvestitionen. Darüber hinaus ist das Haushaltsnettoeinkommen auf dem 1\%-bzw. auf dem 5\%-Niveau signifikant und somit eine wesentliche Determinante dafü, ob eine Person Nichtraucher sowie normalgewichtig ist. Einkommenssorgen hingegen sind für die Männerstichprobe nicht mehr signifikant, wohl aber für die Gesamtstichprobe sowie die der Frauen. Im Querschnitt konnte jeweils ein konkaver Zusammenhang für die Arbeitszeit ermittelt werden. Auch dieser Zusammenhang lässt sich erneut für die Gesamtstichprobe sowie die zweite Schätzung bestätigen, während Arbeitszeit für Frauen nun nicht mehr von Bedeutung ist. Die für das Verhalten der Männer optimale Arbeitszeit liegt nun bei 27,54 Stunden, gegenüber 21,75 Stunden aus der Querschnittsschätzung. Es kann davon ausgegangen werden, dass ein Teil dieses Unterschieds auf die im Panel fehlende Variable Sport zurückzuführen ist, da regelmäßige sportliche Betätigung mit hohem Zeitaufwand einhergeht und so die optimale Arbeitszeit senkt. Während darüber hinaus zumindest einige der Gesundheitsmerkmale im Querschnitt ein signifikantes Vorzeichen aufwiesen, sind diese nun allesamt nicht mehr von Relevanz für das Verhalten.

Um periodenspezifische Effekte abzubilden, wurde darüber hinaus ein Jahresdummy generiert, der für alle Beobachtungen aus dem Jahr 2002 den Wert 1 annimmt. Diese Jahresdummies sind für alle drei Schätzungen nicht signifikant von Null verschieden, so dass keine wesentlichen Unterschiede im Verhalten zwischen den Jahren 2002 und 2004 nachgewiesen werden können. Der Korrelationskoeffizient $\rho$ beträgt jeweils etwa 0,83 und ist stets signifikant positiv. Der Likelihood-Verhältnis-Test (LR Test ${ }^{169}$ ) vergleicht das spezifizierte Modell

${ }^{169}$ Likelihood-Ratio-Test (Chi²-verteilt). 
auf Basis des Maximum-Likelihood-Schätzverfahrens mit einem weiteren Modell, das durch Restriktionen gekennzeichnet ist. Im einfachsten Fall wird untersucht, ob sich durch die verwendeten Variablen eine höhere Aussagekraft erzielen lässt als bei einem Modell, das nur die Konstante enthält.

Tabelle 13: Ergebnisse der Panelschätzung: gesundheitsrelevantes Verhalten Abhängige Variable: gesundheitsrelevantes Verhalten

\begin{tabular}{|c|c|c|c|c|c|c|}
\hline & \multicolumn{2}{|c|}{ (1) ges. Stichprobe } & \multicolumn{2}{|c|}{ (2) nur Mănner } & \multicolumn{2}{|c|}{ (3) nur Frauen } \\
\hline & Koeff. & P-Wert & Koeff. & P-Wert & Koeff. & P-Wert \\
\hline \multicolumn{7}{|l|}{ Jahresdummy } \\
\hline d02 & -0.0254 & 0.468 & $-0,0418$ & 0,400 & $-0,0018$ & 0,974 \\
\hline \multicolumn{7}{|c|}{ Prïdispon. Variablen } \\
\hline Alter & -0.0529 & $0.053^{*}$ & $-0,0619$ & $0,087^{*}$ & $-0,0387$ & 0,370 \\
\hline Alter ${ }^{2}$ & 0.0008 & $0.015^{* *}$ & 0,0010 & $0,031^{* *}$ & 0,0005 & 0,299 \\
\hline Geschlecht & 0.4020 & 0.208 & & & & \\
\hline Interaktion & -0.0041 & 0.592 & & & & \\
\hline Realschule & 0.3585 & $0.001^{* * *}$ & 0,3353 & $0,019 * *$ & 0,3845 & $0,015^{* *}$ \\
\hline FH-Reife & 0.8724 & $0.001 * * *$ & 1,0817 & $0,002 * * *$ & 0,4859 & 0,258 \\
\hline Abitur & 0.6555 & $0.000 * * *$ & 0,8378 & $0,001 * * *$ & 0,5406 & $0,024^{* *}$ \\
\hline Diplom & 1.0083 & $0.000 * * *$ & 1,1976 & $0,000 * * *$ & 0,8679 & $0,000 * * *$ \\
\hline Ausländer & -0.0626 & 0.642 & $-0,3314$ & $0,065^{*}$ & 0,2598 & 0,203 \\
\hline Behinderung & -0.2511 & $0.071^{*}$ & $-0,2722$ & 0,152 & $-0,2071$ & 0,307 \\
\hline \multicolumn{7}{|c|}{ Soziobk. Variablen } \\
\hline Partner_HH & -0.0313 & 0.731 & $-0,1660$ & 0,202 & $-0,1823$ & 0,171 \\
\hline Kind & 0.0102 & 0.895 & 0,1516 & 0,153 & $-0,1698$ & 0,115 \\
\hline Ln_HH-Netto & 0.3138 & $0.000 * * *$ & 0,3647 & $0,002^{* * *}$ & 0,2449 & $0,037 * *$ \\
\hline Eink.-Sorgen & -0.1881 & $0.005^{* * *}$ & $-0,1359$ & 0,146 & $-0,2107$ & $0,027 * *$ \\
\hline PKV & 0.0748 & 0.555 & 0,0113 & 0,944 & 0,1358 & 0,525 \\
\hline PKV_Zusatz & 0.0991 & 0.314 & 0,1260 & 0,371 & 0,0617 & 0,653 \\
\hline \multicolumn{7}{|c|}{ Gesundheitsmerkmale } \\
\hline Anstrengung & -0.1793 & 0.159 & $-0,2826$ & 0,149 & $-0,1072$ & 0,522 \\
\hline Arztbesuche & 0.0019 & 0.827 & 0,0091 & 0,493 & $-0,0043$ & 0,703 \\
\hline $\mathrm{KH}(\mathrm{Vj})$ & -0.0609 & 0.506 & $-0,0563$ & 0,677 & $-0,0722$ & 0,568 \\
\hline$A U(V j)$. & -0.0006 & 0.590 & 0,0011 & 0,551 & $-0,0020$ & 0,206 \\
\hline \multicolumn{7}{|c|}{ Arbeitsmerkmale } \\
\hline Arbeitszeit & 0.0137 & $0.037 * *$ & 0,0246 & $0,005 * * *$ & 0,0061 & 0,572 \\
\hline Arbeitszeit ${ }^{2}$ & -0.0004 & $0.001^{* * *}$ & $-0,0004$ & $0,001 * * *$ & $-0,0003$ & $0,076^{*}$ \\
\hline$\rho$ & 0,8298 & 0.000 & 0,8350 & $0,000 * * *$ & 0,8246 & $0,000 * * *$ \\
\hline Log likelihood & $-6288,60$ & & $-3373,15$ & & $-2900,70$ & \\
\hline $\mathrm{LR} \mathrm{Chi}^{2}$ & 189,37 & $0,000 * * *$ & 135,26 & $0,000^{* * *}$ & 62,01 & $0,000 * *$ \\
\hline $\begin{array}{l}\text { McFadden's } \\
\mathrm{R}^{2} \text { adj. }\end{array}$ & 0,011 & & 0,013 & & 0,002 & \\
\hline $\begin{array}{l}\text { McKelvey } \\
\text { Zavoina } \mathrm{R}^{2}\end{array}$ & 0,212 & & 0,260 & & 0,167 & \\
\hline AIC & 1,685 & & 1,673 & & 1,703 & \\
\hline Beobacht. & 7497 & & 4062 & & 3435 & \\
\hline
\end{tabular}


Für die durchgeführten Schätzungen ist das Ergebnis jeweils signifikant, so dass den gewählten Variablen ein Erklärungsgehalt zukommt und die Variation in der abhängigen Variablen nicht als zufällig angenommen werden kann. ${ }^{170}$ Weiterhin ist der Erklärungsgehalt der gewählten Spezifikation für Männer bei allen drei Gütemaßen etwas besser als für die Stichprobe der Frauen.

\section{Selbst eingeschätzter Gesundheitszustand}

Im Hinblick auf den selbst eingeschätzten Gesundheitszustand ergeben sich gegenüber der Querschnittsschätzung ebenfalls nur wenige Änderungen, die sich zumeist in unterschiedlichen Signifikanzniveaus ausdrücken (siehe Tabelle 14). Darüber hinaus bleiben in der Panelschätzung das Geschlecht mitsamt dem Interaktionsterm sowie Krankenhausaufenthalte der Frauen ohne Einfluss. Die Arbeitszeit spielt in der Gesamtstichprobe ebenfalls keine entscheidende Rolle mehr, wohl aber für die Männer. Die für den Gesundheitszustand optimale Arbeitszeit liegt demnach bei 34,54 Stunden. Damit ist der Unterschiede zu dem Ergebnis der Querschnittsschätzung sehr gering.

Für die Männerstichprobe gilt darüber hinaus, dass im Gegensatz zum Querschnitt nun auch der Einfluss des Einkommens auf dem 5\%-Niveau signifikant positiv ist, so dass ein hohes Einkommen nicht mehr nur das gesundheitsrelevante Verhalten bedingt, sondern auch einen direkten Effekt auf die Gesundheit per se ausübt.

Die Zusammenhänge zwischen dem gesundheitsrelevanten Verhalten auf der einen und der Gesundheit per se auf der anderen Seite werden noch einmal anhand der Gesundheitsindikatoren BMI und Rauchen deutlich. Beide Determinanten sind auch bei der Panelbetrachtung nach wie vor von großer Bedeutung. Die Wahrscheinlichkeit einer optimalen Gesundheit ist für Männer nun bei einem BMI von 21,34 am größten, während sich in der reinen Querschnittsbetrachtung noch ein Optimalwert von 21,66 ergab. Eine deutliche Änderung hat sich hingegen bei dem gesundheitsoptimalen BMI der Frauen ergeben. Im Querschnitt lag dieser noch bei 20,32, im Panel ist der Wert von 23,18 fast drei BMIPunkte darüber und sogar über dem Wert der Männer. Alle Werte liegen gleichermaßen im Bereich der altersunabhängigen Normalgewichtsdefinition.

\footnotetext{
${ }^{170}$ Hierzu wird zunăchst das Modell ohne Restriktionen geschătzt. Anschließend werden sämtliche Variablenparameter auf Null gesetzt. An dieser Stelle sei zudem angemerkt, dass die Wahrscheinlichkeit in den Grenzen Null und Eins liegen muss. Der Logarithmus ist damit stets kleiner Null. Weitere mögliche Testverfahren sind der Wald Test sowie der LagrangeMultiplikator-Test. In Bezug auf die Nullhypothese sind diese Tests asymptotisch aqquivalent zu dem LR-Test (vgl. Greene (2003)).
} 
Tabelle 14: Ergebnisse der Panelschätzung: eingeschätzter Gesundheitszustand Abhängige Variable: Selbst eingeschätzter Gesundheitszustand

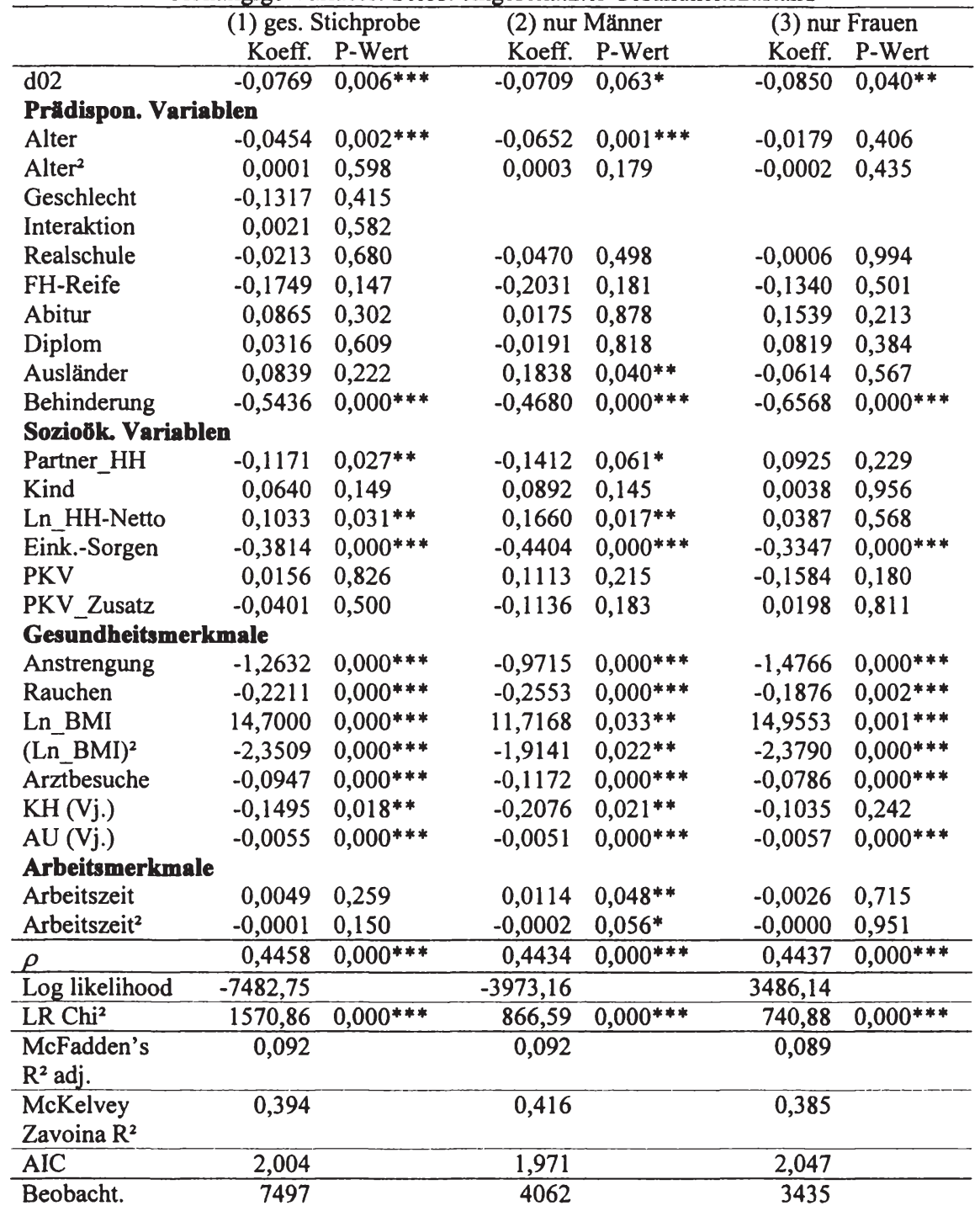

Weiterhin zeigt sich in der Panelschätzung, dass sich die Einschätzung des eigenen Gesundheitszustandes zum Jahr 2004 jeweils verbessert hat, da die Dummies für das Jahr 2002 allesamt signifikant negativ sind. Somit hat sich zwar seit 
2002 keine signifikante Verhaltensänderung eingestellt, wohl aber eine Verbesserung der Gesundheit an sich.

Der Korrelationskoeffizient ist auch für diese drei Schätzungen jeweils ebenso signifikant positiv wie das Ergebnis des LR-Tests. Die Gütemaße des adjustierten Bestimmtheitsmaßes von McFadden sowie des $\mathrm{R}^{2}$ von McKelvey und $\mathrm{Za}$ voina liegen deutlich über denen des gesundheitsrelevanten Verhaltens. Relativ hohe Werte ergeben sich hier insbesondere in dem Maß von McKelvey und Zavoina, da die Anpassungsgüte hier zwischen 0,385 (Frauen) und sogar 0,416 (Männer) schwankt. Demgegenüber steht das AIC, das jeweils für das gesundheitsrelevante Verhalten (vgl. Tabelle 13) niedriger ist, weshalb die Schätzungen letztlich nicht miteinander verglichen werden können.

\subsection{Kritische Würdigung}

Als Ergebnis der ersten drei Panelschätzungen ist festzuhalten, dass insbesondere Bildung, Einkommen und Erwerbstätigkeit das Gesundheitsverhalten entscheidend positiv beeinflussen. Zudem zeigt sich, dass sich die Einflussfaktoren des Gesundheitsverhaltens auch auf den Gesundheitszustand auswirken. Somit fuhren Maßnahmen, die das Verhalten positiv beeinflussen, gleichzeitig zu einer besseren Gesundheit.

Bemerkenswert sind diese Zusammenhänge insbesondere im Hinblick auf die Inanspruchnahme medizinischer Leistungen. Diese wirken sich zwar eindeutig auf den Gesundheitszustand aus, bringen für das Verhalten jedoch keine Lenkungswirkung mit sich, da die Variablen Arztbesuche und $K H(V j$.) jeweils im Panel nicht signifikant sind. Im Querschnitt hat sich zumindest ein positiver Einfluss von Arztbesuchen für die Männer ergeben, Krankenhausaufenthalte im Vorjahr waren für Frauen dort sogar signifikant negativ. Um eine Verbesserung der Gesundheit zu erzielen, können folglich nicht nur die direkten Gesundheitsleistungen ausgeweitet werden, sondern ein Einwirken auf das Verhalten über ausreichende Beratung würde sich ebenfalls positiv auf die Gesundheit auswirken.

Die genannten Merkmale Bildung, Einkommen und Arbeit sind sämtlich Determinanten der sozialen Schichtzugehörigkeit. Damit können die im theoretischen Teil dieser Arbeit untersuchten Spiralwirkungen der empirischen Überprüung standhalten. Erstens führt eine gute Gesundheit insbesondere in der Kindheit zu mehr Bildung, woraus sich positive Effekte hinsichtlich des zukünftigen gesundheitsrelevanten Verhaltens ergeben. Zweitens bedingen ein hoher Bildungsstand sowie gute Gesundheit eine hohe Arbeitsproduktivität, wodurch technischer Fortschritt sowie Wirtschaftswachstum erzielt werden können. Mit dem 
Wirtschaftswachstum gehen wiederum sowohl ein steigendes Einkommen als auch ein hoher Beschäftigungsgrad einher, so dass sich auch hier positive Konsequenzen für die Gesundheit ergeben. Erneut stellt sich damit eine Spiralwirkung ein. Drittens kann die durchschnittliche Arbeitszeit bei mehr Beschäftigung gesenkt werden, indem es zu weniger Überstunden kommt, die reguläre Erwerbstätigkeit hingegen ausgeweitet wird. Letztlich können so mehr Individuen von den positiven Effekten der Arbeit profitieren, während die negativen Auswirkungen beschränkt werden können. 


\subsection{Anhang zu Kapitel 8}

A Einführung in Modelle mit binären und kategorialen Variablen in Querschnittsanalysen

\section{A.1 Binäre Variablen: Das lineare Wahrscheinlichkeitsmodell}

Von besonderer Relevanz für die nachfolgende Analyse ist die Verwendung einer binären Variablen als abhängige Variable. ${ }^{171}$ Das Strukturmodell hierzu lautet

$$
y=\beta_{0}+\beta_{1} x_{1}+\ldots+\beta_{k} x_{k}+\varepsilon=\boldsymbol{x} \boldsymbol{\beta}+\varepsilon,
$$

wobei $\boldsymbol{x}$ der Vektor aller erklärenden Variablen ist. $\boldsymbol{\beta}$ bezeichnet den Parametervektor, $\varepsilon$ den Fehlerterm. Besitzt der Störterm $\varepsilon$ einen Erwartungswert von Null, dann gilt für den Erwartungswert von $y$

$$
E(y \mid x)=\boldsymbol{x} \beta
$$

Gleichzeitig lässt sich der Erwartungswert als

$$
E(y \mid \boldsymbol{x})=[1 \times P(y=1 \mid \boldsymbol{x})]+[0 \times P(y=0 \mid \boldsymbol{x})]=P(y=1 \mid \boldsymbol{x})
$$

schreiben. Damit ist der Erwartungswert von $y$ unter der Bedingung von $\boldsymbol{x}$ gleichzeitig der Wahrscheinlichkeit, dass $y$ bei gegebenem $\boldsymbol{x}$ den Wert 1 annimmt. Die Schätzung der binären Variablen erfolgt unter Anwendung des $\mathrm{OLS}^{172}$-Regressionsmodells. Die Bezeichnung dieses Modells als lineares Wahrscheinlichkeitsmodell bezieht sich auf die Tatsache, dass $\beta_{j}$ die Wahrscheinlichkeit angibt, mit der sich $y$ ändert, wenn sich $x_{j}$ bei Konstanz aller übrigen Faktoren ändert.

171 Einen Sonderfall der ordinal skalierten Variablen stellen binäre Variablen oder auch Dummy-Variablen dar. Hierbei handelt es sich um Variablen, die stets ausschließlich die Werte Null und Eins annehmen und damit lediglich zwei Kategorien aufweisen. Derartige qualitative Variablen beschreiben einen Entweder-oder-Zusammenhang: männlich oder weiblich, erwerbstătig oder Rentner, usw. Für die Verwendung von Dummy-Variablen ist es daher maßgeblich, nach einem Ereignis zu fragen, bei dessen Eintreten der Variablen der Wert Eins für „zutreffend“ zugeordnet wird. Das Gegenereignis erhält entsprechend den Wert Null (vgl. hierzu Wooldridge (2003), S. 240ff.).

172 OLS $=$ Ordinary Least Squares 
Das Ergebnis eines Regressionsmodells mit einer metrisch skalierten abhängigen Variablen lässt sich derart interpretieren, dass der Koeffizient $\beta_{j}$ die Veränderung von $y$ aufgrund einer Veränderung von $x_{j}$ um eine Einheit angibt unter der Prämisse, dass alle übrigen Variablen konstant gehalten werden. Eine solche Interpretation ist bei binären Variablen nicht mehr möglich, da $y$ lediglich die Ausprägungen Null und Eins besitzt und damit auch nur Veränderungen von Null auf Eins oder von Eins auf Null möglich sind.

Auch wenn das lineare Wahrscheinlichkeitsmodell in seiner Interpretation dem OLS-Modell ähnlich ist, so sind doch einige der Standardannahmen verletzt (vgl. Long und Freese (2003), S. 38ff.). Die erste verletzte Annahme besteht in dem Problem der Heteroskedastie. Die Varianz von $y$ gegeben $\boldsymbol{x}$ errechnet sich durch $\operatorname{Var}(y \mid \boldsymbol{x})=\mathrm{E}\left[(y-\mathrm{E}(y))^{2}\right]$. Durch Einsetzen von (8.6) folgt hierfür $\operatorname{Var}(y \mid x)=\mathrm{E}\left(y^{2}\right)-2 \mathrm{P}(y=1 \mid \boldsymbol{x}) \mathrm{E}(y)+[\mathrm{P}(y=1 \mid \boldsymbol{x})]^{2}$. Da y lediglich die Ausprägungen 0 und 1 annehmen kann, wird $\mathrm{E}\left(y^{2}\right)$ zu $\mathrm{E}(y)=\mathrm{P}(y=1 \mid \boldsymbol{x})$. Die Varianz von $y$ lässt sich damit durch

$$
\operatorname{Var}(y \mid \boldsymbol{x})=P(y=1 \mid \boldsymbol{x})[1-P(y=1 \mid \boldsymbol{x})]
$$

bestimmen. Diese Darstellung zeigt, dass die Varianz von den Wahrscheinlichkeiten der beiden Ereignisse und damit direkt von $\boldsymbol{x}$ abhängt, so dass diese nicht mehr konstant ist. Der OLS-Schätzer ist entsprechend ineffizient und die Standardfehler sind verzerrt. Damit ist die Teststatistik nicht mehr korrekt, was auch für die Konfidenzintervalle gilt.

Zum zweiten ist die Annahme eines normalverteilten Störterms verletzt. Da $y$ nur die Werte Null und Eins annehmen kann, folgt hieraus für den Störterm, dass dieser entweder $\boldsymbol{x} \boldsymbol{\beta}$ oder $1-\boldsymbol{x} \boldsymbol{\beta}$ betragen muss. ${ }^{173}$ Hieraus ergibt sich auch die Möglichkeit unsinniger Wahrscheinlichkeitsergebnisse, denn der Störterm kann nicht auf das 0-1-Intervall beschränkt werden. Für besonders niedrige Werte von $x$ werden beispielsweise negative Wahrscheinlichkeiten errechnet, während besonders hohe Werte dazu führen können, dass die prognostizierte Wahrscheinlichkeit den Wert 1 übersteigt.

Die letzte Kritik bezieht sich auf die Linearität des Wahrscheinlichkeitsmodells. Der Einfluss eines Faktors auf die Wahrscheinlichkeit ist im Modell stets konstant, jedoch sind in der Realität häufig abnehmende Effekte zu beobachten, je weiter sich die Wahrscheinlichkeit in Richtung der Extremwerte Null und Eins

\footnotetext{
${ }^{173}$ Die Bedingung eines normalverteilten Störterms ist jedoch nicht zwingend notwendig, um unverzerrte OLS-Schätzer zu erhalten (vgl. Long und Freese (2003), S. 39).
} 
bewegt. So ist beispielsweise davon auszugehen, dass die Entscheidung über die Berufstätigkeit von Frauen stärker von der Geburt des ersten Kindes als von der Geburt weiterer Kinder abhängt. Das lineare Wahrscheinlichkeitsmodell sagt jedoch für alle Kinderzahlen einen konstanten Effekt voraus, d. h. jedes zusätzliche Kind verringert die Wahrscheinlichkeit der Erwerbstätigkeit um den gleichen Betrag (vgl. Long und Freese (2003), S. 39f.).

\section{A.2 Probit und Logit Modelle für binäre Variablen}

Eine alternative Betrachtungsweise des Problems besteht in der Annahme, dass der beobachteten Variablen $y$ eine latente, $d$. h. nicht beobachtbare, stetige Variable $y^{*}$ zugrunde liegt. Beobachtbar ist lediglich, ob $y$ den Wert 0 oder den Wert 1 annimmt, Informationen über das Zustandekommen dieser Werte sind jedoch nicht vorhanden. Das Konzept der latenten Variablen sieht nun vor, dass es einen Schwellenwert $\tau$ geben muss, bei dessen Überschreiten eine Veränderung der Entscheidung herbeigeführt wird. Im weiteren Verlauf wird angenommen, dass dieser Schwellenwert exakt bei $\tau=0$ liegt (vgl. hierzu Long (1997), S.41ff.):

$$
y_{j}=\left\{\begin{array}{ll}
1 & \text { für } y_{j}^{*}>\tau \\
0 & \text { für } y_{j}^{*} \leq \tau
\end{array} .\right.
$$

Als Beispiel hierzu führt Long (1997) die Erwerbstätigkeit von Frauen an. Tatsächlich beobachtet werden kann nur die Tatsache, ob eine Frau erwerbstätig ist oder nicht. Nicht beobachtbar ist jedoch, mit welcher Sicherheit diese Entscheidung vertreten wird. So kann es sein, dass eine Frau kurz davor ist, ihre Stelle zu kündigen, während eine andere fest in ihrem Job verankert ist. Für beide Personen wird die gleiche Ausprägung von $y$ beobachtet.

Wird nun das Modell der latenten Variablen durch

$$
y^{*}=\boldsymbol{x} \boldsymbol{\beta}+\varepsilon
$$

beschrieben, wird deutlich, dass diese unbeobachteten Unterschiede über den Störterm aufgefangen werden müssen. Analog zum linearen Wahrscheinlichkeitsmodell wird dem Störterm ein Erwartungswert von Null unterstellt $(E(\varepsilon \mid x)=0)$, d. h. der Störterm ist von $\boldsymbol{x}$ unabhängig. Die Varianz des Störterms ist nicht berechenbar, da es sich bei $y^{*}$ um eine nicht beobachtbare Variable handelt. Das Probit-Modell unterstellt daher eine Standardnormalverteilung des Störterms, während das Logit-Modell eine logistische Verteilung annimmt. Da- 
mit gilt entweder $\operatorname{Var}(\varepsilon \mid \boldsymbol{x})=1$ im Probit-Modell oder $\operatorname{Var}(\varepsilon \mid \boldsymbol{x})=\pi^{2} / 3$ im Logit-Modell.

Für einen normalverteilten Störterm mit $E(\varepsilon \mid \boldsymbol{x})=0$ und $\operatorname{Var}(\varepsilon \mid \boldsymbol{x})=1$ ergibt sich eine Wahrscheinlichkeitsdichte von

$$
\phi(\varepsilon)=\frac{1}{\sqrt{2 \pi}} \exp \left(-\frac{\varepsilon^{2}}{2}\right)
$$

Die kumulative Verteilungsfunktion ${ }^{174}$ wird bestimmt durch

$$
\Phi(\varepsilon)=\int_{-\infty}^{\varepsilon} \frac{1}{\sqrt{2 \pi}} \exp \left(-\frac{t^{2}}{2}\right) d t
$$

Die Varianz der Logit-Verteilung von $\pi^{2} / 3$ ergibt eine möglichst einfache Wahrscheinlichkeitsdichte (vgl. Long (1997), S. 42):

$$
\lambda(\varepsilon)=\frac{\exp (\varepsilon)}{[1+\exp (\varepsilon)]^{2}}
$$

Für die Verteilungsfunktion gilt

$$
\Lambda(\varepsilon)=\frac{\exp (\varepsilon)}{1+\exp (\varepsilon)}
$$

Bei beiden Verteilungen handelt es sich um ansteigende Funktionen. Zudem gilt für beide gleichermaßen, dass die Steigung bei $\varepsilon=0$ am größten ist. Weiterhin gilt: Geht der Störterm gegen $-\infty$, nähert sich die Wahrscheinlichkeit dem Wert 0 , während ein Störterm von $\varepsilon \rightarrow \infty$ zu einer Wahrscheinlichkeit von Eins führt (vgl. Wooldridge (2003), S. 554ff.).

${ }^{174}$ Diese gibt die Wahrscheinlichkeit dafür an, dass eine Zufallsvariable kleiner oder gleich einem bestimmten Wert ist (vgl. Long (1997), S. 42). 
Diese Zusammenhänge lassen sich wie folgt erläutern: Angenommen, zwei Individuen stehen vor der gleichen Kaufentscheidung. Die erste Person besitzt eine hohe Kaufneigung, während der zweiten Person anhand der beobachteten Charakteristika lediglich eine geringe Kaufneigung zugeordnet wird. Nun treffen beide Individuen dennoch eine positive Kaufentscheidung. Bei dem ersten Individuum lässt sich diese Entscheidung direkt durch die unabhängigen Variablen erklären, während bei dem zweiten Individuum Einflussfaktoren existieren müssen, welche die Kaufentscheidung erklären. Diese Faktoren werden über einen hohen positiven Störterm aufgefangen (vgl. Kennedy (2003), S. 261).

Die Unterschiede zwischen dem Logit-Modell auf der einen und dem ProbitModell auf der anderen Seite lassen sich anhand der folgenden Abbildung 19 erläutern. Die Wahrscheinlichkeitsdichte der logistischen Verteilung weist eine höhere Varianz auf als die Normalverteilung, so dass der gesamte Kurvenverlauf flacher ist. Für die vorhergesagten Wahrscheinlichkeiten, dargestellt im zweiten Diagramm der Abbildung, bedeutet dies eine langsamere Annäherung an die Ränder. Somit werden bei einem Störterm kleiner Null vergleichsweise höhere Wahrscheinlichkeiten errechnet, während ein positiver Störterm zu eher niedrigeren Wahrscheinlichkeiten führt (vgl. hierzu Greene (2003), S. 667).

Die Ergebnisse beider Modelle sind gleichermaßen interpretierbar. Daher stellt sich die Frage, welches Modell am besten für die Schätzproblematik gewählt werden sollte. Aufgrund der relativ einfachen Struktur wurde in der Vergangenheit häufig das Logit-Modell vorgezogen, doch dieser Vorteil ist mit zunehmend leistungsfähigeren Computerprogrammen nicht mehr ausschlaggebend. Vielmehr bezeichnet Kennedy (2003) die Wahl des Modells als eine Frage des eigenen Geschmacks. Wooldridge (2003) hingegen sieht das Probit-Modell als das populärere an, da eine Normalverteilung des Störterms von Ökonometrikern allgemein bevorzugt wird (vgl. Wooldridge (2003), S. 556).

Tatsächlich liefern beide Verfahren ähnliche Ergebnisse, solange nur wenige Stichprobenwerte an den Rändern liegen. Unterschiedliche Wahrscheinlichkeiten bekommt man dagegen insbesondere dann, wenn eine der Antwortmöglichkeiten 0 oder 1 besonders häufig enthalten ist oder wenn eine unabhängige Variable eine hohe Streuung aufweist. ${ }^{175}$

Aus den genannten Gründen sind die Ergebnisse der Parameterschätzungen $\boldsymbol{\beta}$ nicht direkt miteinander vergleichbar. Zumindest eine Annäherung der Parame-

${ }^{175} \mathrm{Zu}$ weiteren Details hinsichtlich der Verwendung von Probit- und Logit-Modellen siehe Amemiya (1981). 
ter erhält man, wenn die Schätzwerte von $\boldsymbol{\beta}$, die das Logit-Modell liefert, mit $3^{1 / 2} / \pi$ multipliziert werden (vgl. Maddala (1999), S. 23).

Abbildung 19: Normalverteilung und Logistische Verteilung
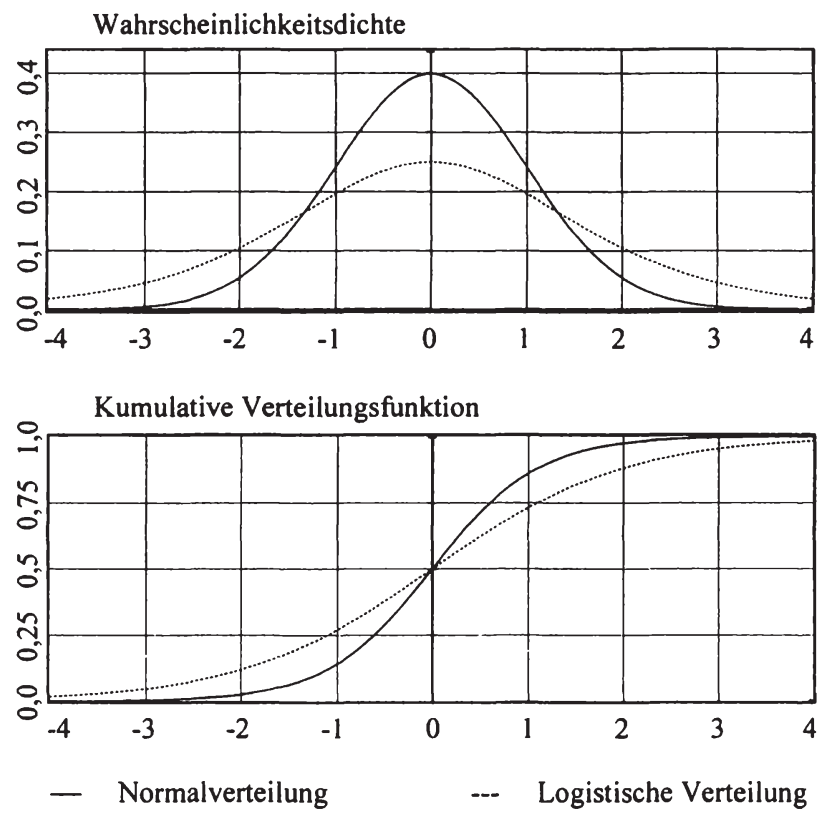

Quelle: In Anlehnung an Long (1997), S. 43.

Aufgrund der Stetigkeit der latenten Variablen treten die Probleme des linearen Wahrscheinlichkeitsmodells nicht weiter auf, allerdings ist auch keine OLSSchätzung mehr möglich. Stattdessen wird eine Maximum-Likelihood-Schätzung angewendet. Das Ergebnis ist entsprechend die Wahrscheinlichkeit, dass ein bestimmtes Ereignis eintritt, und der Wert 1 minus dieser Wahrscheinlichkeit steht für das Nicht-Eintreten des Ereignisses. Diese Werte werden über alle Beobachtungen miteinander multipliziert.

Aus Bedingung (8.8) folgt für einen Schwellenwert von Null, dass die abhängige Variable $y$ den Wert Eins annimmt, sobald die latente Variable $y^{*}$ größer Null ist. Damit gilt auch für die Wahrscheinlichkeiten, dass 


$$
P(y=1 \mid \boldsymbol{x})=P\left(y^{*}>0 \mid \boldsymbol{x}\right)
$$

sein muss. Aus (8.9) folgt wiederum

$$
P(y=1 \mid \boldsymbol{x})=P(\boldsymbol{x} \boldsymbol{\beta}+\varepsilon>0 \mid \boldsymbol{x})
$$

bzw.

$$
P(y=1 \mid \boldsymbol{x})=P(\varepsilon>-\boldsymbol{x} \boldsymbol{\beta} \mid \boldsymbol{x}) .
$$

Aufgrund der Symmetrie, die für beide Verteilungen gleichermaßen zutriff, lässt sich Gleichung (8.16) auch in Form von

$$
P(y=1 \mid \boldsymbol{x})=P(\varepsilon \leq \boldsymbol{x} \boldsymbol{\beta} \mid \boldsymbol{x})
$$

schreiben. Bei diesem Ausdruck handelt es sich um die kumulative Verteilungsfunktion des Fehlers an der Stelle $\boldsymbol{x} \boldsymbol{\beta}$. Damit ist

$$
P(y=1 \mid \boldsymbol{x})=F(\boldsymbol{x} \boldsymbol{\beta}),
$$

wobei $F$ die normalverteilte Funktion $\Phi$ für das Probit-Modell und die logistisch verteilte Funktion $\Lambda$ im Fall des Logit-Modells darstellt (vgl. zu diesem Abschnitt Long (1997), S. 45). Dieser Zusammenhang fuhrt zu folgender Likelihood-Funktion, die die Wahrscheinlichkeiten für ein Ereignis mit den Gegenwahrscheinlichkeiten in Beziehung setzt:

$$
\begin{gathered}
P\left(Y_{1}=y_{1}, Y_{2}=y_{2}, \ldots, Y_{n}=y_{n} \mid \boldsymbol{X}\right) \\
\quad=\prod_{y_{i}=0}\left[1-F\left(\boldsymbol{x}_{i} \boldsymbol{\beta}\right)\right] \prod_{y_{i}=1} F\left(\boldsymbol{x}_{i} \boldsymbol{\beta}\right) .
\end{gathered}
$$

Diese Likelihood-Funktion kann auch als

$$
L=\prod_{i=1}^{n} F\left(\boldsymbol{x}_{i} \boldsymbol{\beta}\right)^{y_{i}}\left[1-F\left(\boldsymbol{x}_{i} \boldsymbol{\beta}\right)\right]^{1-y_{i}}
$$


geschrieben werden. Um nun das Maximum zu erhalten, wird der Ausdruck zunächst logarithmiert:

$$
\ln L=\sum_{i=1}^{n}\left\{y_{i} \ln F\left(\boldsymbol{x}_{i} \boldsymbol{\beta}\right)+\left(1-y_{i}\right) \ln \left[1-F\left(\boldsymbol{x}_{i} \boldsymbol{\beta}\right)\right]\right\} .
$$

Anschließend erfolgt die Ableitung nach dem Parametervektor $\boldsymbol{\beta}$.

$$
\frac{\partial \ln L}{\partial \beta}=\sum_{i=1}^{n}\left[y_{i} \frac{f_{i}}{F_{i}}-\left(1-y_{i}\right) \frac{f_{i}}{1-F_{i}}\right] \boldsymbol{x}_{i}=0 \text {. }
$$

$f_{i}$ ist die Ableitung der Verteilungsfunktion $F_{i}, d F_{i} / d(\boldsymbol{x} \boldsymbol{\beta})$, und kann damit durch die entsprechende Dichtefunktion der Normalverteilung oder der logistischen Verteilung ersetzt werden. Im Fall der logistischen Verteilungsfunktion gilt damit in Analogie zu Gleichung (8.13) ${ }^{176}$

$$
\Lambda(\boldsymbol{x} \beta)=\frac{\exp (\boldsymbol{x} \boldsymbol{\beta})}{1+\exp (\boldsymbol{x} \boldsymbol{\beta})}
$$

sowie für die Dichtefunktion

$$
\lambda(\boldsymbol{x} \boldsymbol{\beta})=\frac{\exp (\boldsymbol{x} \boldsymbol{\beta})}{[1+\exp (\boldsymbol{x} \boldsymbol{\beta})]^{2}} .
$$

Durch Einsetzen der Gleichungen (8.23) und (8.24) in die Likelihood-Gleichung (8.22) erhält man schließlich die Likelihood-Funktion für das Logit-Modell (vgl. im folgenden Maddala (1999), S. 25f. sowie Greene (2003), S. 671):

$$
\frac{\partial \ln L}{\partial \boldsymbol{\beta}}=\sum_{i=1}^{n}\left(y_{i}-\Lambda_{i}\right) \boldsymbol{x}_{i}=0
$$

${ }^{176}$ Vgl. hierzu Maddala (1999), S. 22. 
Für das Probit-Modell wird $F\left(\boldsymbol{x}_{i} \beta\right)$ in Gleichung (8.20) und (8.21) entsprechend ersetzt durch die Verteilungsfunktion $\Phi\left(x_{i} \beta\right)$. Damit folgt für die Ableitung der logarithmierten Likelihood-Funktion (8.21) nach dem Parametervektor $\beta$ :

$$
\frac{\partial \ln L}{\partial \beta}=\sum_{i=1}^{n} \frac{y_{i}-\Phi\left(x_{i} \beta\right)}{\Phi\left(x_{i} \beta\right)\left[1-\Phi\left(x_{i} \beta\right)\right]} \phi\left(x_{i} \beta\right) x_{i}=0
$$

Somit zeigt sich, dass sich die jeweiligen Modelle einzig durch die unterstellte Verteilung unterscheiden. Da diese jedoch nicht bekannt ist, kann keine eindeutige Empfehlung fur die Verwendung des einen oder des anderen Modells ausgesprochen werden.

\section{A.3 Kategoriale Variablen}

Besitzt die abhängige Variable nicht nur zwei, sondern mehrere Ausprägungen, die nach einem bestimmten Schema geordnet werden können, spricht man von kategorialen Variablen. Als Beispiel hierfür lassen sich sämtliche Fragen anführen, bei denen die Antwort anhand einer Skala gegeben wird. Jedem Zahlenwert, beispielsweise auf einer Skala von 1 bis 10, wird eine Interpretation zugeordnet, wobei die Intensität in der Regel von der Mitte zu den Rändern hin zunimmt. Wird jemand um seine Meinung gebeten, so führt die Skala von ,stimme überhaupt nicht zu“ am unteren Rand bis hin zu ,stimme vollkommen zu“ am oberen Rand. Die Berechnung der Wahrscheinlichkeiten erfolgt nun über so genannte Ordered Probit- bzw. Ordered Logit-Modelle. Da es zunächst nicht von Bedeutung ist, ob nun das Probit- oder das Logit-Modell gewählt wird, werden diese nachfolgend als Modelle für geordnete Kategorien zusammengefasst.

Im Rahmen der empirischen Untersuchung ist der Einfluss der Erwerbstätigkeit auf das gesundheitsrelevante Verhalten bzw. die Gesundheit per se von besonderem Interesse, indem hierüber das Einkommen sowie die verfügbare Zeit determiniert werden. Beide Variablen werden durch eine Ordinalskala abgebildet. ${ }^{177}$ Tatsächlich handelt es sich jedoch sowohl beim gesundheitsrelevanten Verhalten als auch bei der Gesundheit um latente Variablen, die nicht genau beobachtet werden können. Daher wird das Konzept der latenten Variablen bei 0-1-Entscheidungen auf Variablen mit mehreren Kategorien ausgeweitet. Erneut gilt, dass der Übergang von einer Kategorie zur nächsten durch das Überschreiten eines nicht beobachtbaren Schwellenwertes modelliert wird. Bezeichnet nun $y$

${ }^{177}$ Eine genaue Beschreibung der verwendeten Variablen erfolgt in den Abschnitten 8.1.2 und 8.2.2. 
die beobachtete Variable und $y^{*}$ die latente Variable (siehe Gleichung (8.9)), dann lässt sich die Zuordnung zu den Variablenkategorien wie folgt charakterisieren: ${ }^{178}$

$$
y=m \quad \text { für } \tau_{m-1} \leq y^{*}<\tau_{m}, \quad m=1, \ldots, J .
$$

Die untere Grenze $\tau_{0}$ liegt bei $-\infty$, die obere $\tau_{J}$ bei $+\infty$. Für eine Kategorienanzahl von $J=2$ entspricht dieser Ansatz genau dem in Gleichung (8.8) geschilderten Prinzip.

Sowohl im Probit- als auch im Logit-Modell entsprechen die Dichte- und Verteilungsfunktionen denen für binäre Variablen, so dass die Gleichungen (8.10) bis (8.13) nach wie vor Gültigkeit besitzen. Nachfolgend wird daher erneut die Funktion $F$ für $\Phi$ bzw. $\Lambda$ und die Funktion $f$ für $\phi$ oder $\lambda$ verwendet.

Angenommen, die erste Kategorie wird mit der Ziffer 0 bezeichnet, so dass $y=0$ gilt, wenn $y^{*}$ zwischen $\tau_{0}=-\infty$ und $\tau_{1}$ liegt. Für die Wahrscheinlichkeit, dass $y=0$ ist, folgt dann

$$
P(y=0 \mid x)=P\left(\tau_{0} \leq y^{*}<\tau_{1} \mid x\right) .
$$

Substituiert man $y^{*}$ durch die Strukturgleichung (8.9), lässt sich Gleichung (8.28) in Form von

$$
P(y=0 \mid \boldsymbol{x})=P\left(\tau_{0} \leq \boldsymbol{x} \boldsymbol{\beta}+\varepsilon<\tau_{1} \mid \boldsymbol{x}\right)
$$

darstellen. Durch Subtraktion von $\boldsymbol{x} \boldsymbol{\beta}$ verändert sich Gleichung (8.29) schließlich zu

$$
P(y=0 \mid x)=P\left(\tau_{0}-x \boldsymbol{\beta} \leq \varepsilon<\tau_{1}-\boldsymbol{x} \boldsymbol{\beta} \mid \boldsymbol{x}\right) .
$$

Gesucht wird gemäß dieser Gleichung nach der Wahrscheinlichkeit, dass die Zufallsvariable $\varepsilon$ zwischen zwei bestimmten Werten liegt. Dieser Zusammenhang ist wiederum identisch mit der Differenz aus der Wahrscheinlichkeit, dass der Wert von $\varepsilon$ unterhalb der Obergrenze liegt und der Wahrscheinlichkeit, dass $\varepsilon$ unterhalb der Untergrenze liegt. Diese Wahrscheinlichkeiten können wie-

${ }^{178}$ Vergleiche hierzu sowie zu den folgenden Ausführungen Long (1997), S. 116-124. 
derum durch die Verteilungsfunktionen substituiert werden, so dass die allgemeine Formulierung von (8.30) durch

$$
P(y=m \mid \boldsymbol{x})=F\left(\tau_{m}-\boldsymbol{x} \boldsymbol{\beta}\right)-F\left(\tau_{m-1}-\boldsymbol{x} \boldsymbol{\beta}\right)
$$

gegeben ist.

Die Likelihood-Funktion ist damit

$$
L(\boldsymbol{\beta}, \tau \mid \boldsymbol{y}, \boldsymbol{X})=\prod_{m=1}^{J} \prod_{y_{i}=j}\left[F\left(\tau_{m}-\boldsymbol{x}_{i} \boldsymbol{\beta}\right)-F\left(\tau_{m-1}-\boldsymbol{x}_{i} \boldsymbol{\beta}\right)\right]
$$

Durch logarithmieren erhält man schließlich die zugehörige Log-LikelihoodFunktion:

$$
\ln L(\boldsymbol{\beta}, \boldsymbol{\tau} \mid \boldsymbol{y}, \boldsymbol{X})=\sum_{m=1}^{J} \sum_{y_{i}=j} \ln \left[F\left(\tau_{m}-\boldsymbol{x}_{i} \boldsymbol{\beta}\right)-F\left(\tau_{m-1}-\boldsymbol{x}_{i} \boldsymbol{\beta}\right)\right]
$$

Die Parametervektoren $\boldsymbol{\tau}$ und $\boldsymbol{\beta}$ können mit Hilfe des Maximum LikelihoodSchätzverfahrens geschätzt werden. Im Fall des Ordered Probit-Modells wird $F$ durch $\Phi$ ersetzt, während $F$ im Ordered-Logit-Modell durch die Verteilungsfunktion $\Lambda$ substituiert wird. In der folgenden ökonometrischen Untersuchung findet jedoch ausschließlich das Ordered Probit-Modell Anwendung.

\section{A.4 Gütemaße}

Im linearen Regressionsmodell gibt das Bestimmtheitsmaß $R^{2}$ denjenigen Anteil der Variation in der abhängigen Variablen an, der durch die Regressoren erklärt werden kann (vgl. zu diesem Abschnitt insbesondere Long (1997), S. 102-110). Hierzu wird die durch das Modell erklärte Varianz zur Gesamtvarianz der abhängigen Variablen in Beziehung gesetzt. Die Gesamtvarianz TSS ${ }^{179}$ ist gleich der Summe der quadratischen Abweichungen der beobachteten Werte $Y_{i}$ von ihrem arithmetischen Mittel $\bar{Y}$. Derjenige Teil der Varianz, der nicht durch die Variablen erklärt wird, wird mit $R S S^{180}$ bezeichnet. Dieser Wert entspricht der Summe der quadratischen Abweichungen der Fehler, die sich aus der Differenz

${ }^{179} T S S=$ Total Sum of Squares
${ }^{180} R S S=$ Residual Sum of Squares 
der beobachteten Werte $Y_{i}$ und dem bedingten Erwartungswert $\hat{Y}=x \hat{\beta}$ ergibt. Für das Bestimmtheitsmaß $R^{2}$ folgt hieraus

$$
R^{2}=\frac{T S S-R S S}{T S S}=1-\frac{R S S}{T S S}=1-\frac{\sum_{i=1}^{N}\left(y_{i}-\hat{y}_{i}\right)^{2}}{\sum_{i=1}^{N}\left(y_{i}-\bar{y}\right)^{2}} .
$$

Nun gilt dieses Maß für Regressionsmodelle mit stetigen Variablen. Sobald es sich jedoch um binäre oder ordinal skalierte Variablen handelt, ist ein anderes Bestimmtheitsmaß notwendig, da die Varianz nicht geschätzt werden kann. Im Gegensatz zum allgemein akzeptierten $R^{2}$ gibt es für Probit-Modelle jedoch kein allgemeingültiges Bestimmtheitsmaß (vgl. Kennedy (2003), S. 267). Vielmehr existiert eine Vielzahl von Ansätzen, von denen an dieser Stelle nur einige genannt werden sollen. ${ }^{181}$

Das Pseudo- $\mathrm{R}^{2}$ von McFadden ist für jedes Maximum-Likelihood-Modell gültig. Hierfür wird zunächst die Log Likelihood eines Modells ohne Regressoren $M_{\alpha}$ bestimmt, die anstelle der Gesamtvarianz verwendet wird. Der Wert der Residuen $R S S$ wird durch die Log Likelihood des gesamten Modells mit Regressoren, $M_{\beta}$ ersetzt. Damit ergibt sich in Analogie zu Gleichung (8.34)

$$
R_{M c F}^{2}=1-\frac{\ln \hat{L}\left(M_{\beta}\right)}{\ln \hat{L}\left(M_{\alpha}\right)}
$$

Dem $\mathrm{R}^{2}$ von McFadden sind zwei Besonderheiten zuzuschreiben. Erstens ist es nicht möglich, dass dieses $\mathrm{Ma}$ den Wert 1 annimmt, und zweitens ist es umso höher, je mehr Variablen enthalten sind. Um diese Auswirkungen zu vermeiden, wird in dem korrigierten Bestimmtheitsmaß $\bar{R}^{2}{ }_{M c F}$ die Zahl der im Modell enthaltenen Parameter $K$ berücksichtigt, so dass sich Gleichung (8.35) zu

$$
\bar{R}_{M c F}^{2}=1-\frac{\ln \hat{L}\left(M_{\beta}\right)-K}{\ln \hat{L}\left(M_{\alpha}\right)}
$$

${ }^{181}$ Weitere Bestimmtheitsmaße finden sich bei Long (1997). 
verändert. In diesem Fall steigt das Bestimmtheitsmaß nur dann, wenn $\ln \hat{L}\left(M_{\beta}\right)$ um mehr als 1 ansteigt, sobald ein neuer Regressor hinzugefügt wird. Durch diese Vorgehensweise bedeutet ein höherer Wert des Bestimmtheitsmaßes auch eine bessere Anpassung. Eine genaue Interpretation des Ergebnisses ist jedoch nach wie vor nicht möglich, da es keinen Standard darüber gibt, bei welchem Wert tatsächlich von einer hohen Anpassung auszugehen ist.

Ein Pseudo- $\mathrm{R}^{2}$ für latente Variablen liefern McKelvey und Zavoina (1975). Hier wird die geschätzte Varianz der latenten Variablen, dargestellt im Zähler von Gleichung (8.37), zur tatsächlichen Varianz in Beziehung gesetzt:

$$
R_{M \& Z}^{2}=\frac{\hat{\operatorname{Var}}\left(\hat{y}^{*}\right)}{\operatorname{Var}\left(y^{*}\right)}=\frac{\hat{\operatorname{Var}}\left(\hat{y}^{*}\right)}{\operatorname{Var}\left(\hat{y}^{*}\right)+\operatorname{Var}(\varepsilon)} .
$$

Die Varianz des Störterms $\varepsilon$ ist fix durch das Schätzverfahren vorgegeben. Für das Probit-Modell gilt $\operatorname{Var}(\varepsilon)=1$. Die Varianz der unbeobachtbaren Variablen lässt sich hingegen über die Kovarianzmatrix der erklärenden Variablen bestimmen (vgl. hierzu Long (1997)).

Veall und Zimmermann (1992) und (1996) untersuchen anhand von Simulationstests, welches der genannten Pseudo- $\mathrm{R}^{2}$ dem OLS- $\mathrm{R}^{2}$ am nächsten kommt. Hierzu wird eine abhängige stetige Variable in ordinal skalierte Variablen mit zwei, drei und vier Kategorien umgewandelt. Es zeigt sich, dass das Pseudo- $\mathbf{R}^{2}$ von McKelvey und Zavoina dem $R^{2}$ für stetige Variablen am nächsten kommt.

Akaikes Informationskriterium (AIC ${ }^{182}$ ) schließlich verwendet ebenfalls den Log Likelihood-Schätzer des gesamten Modells, setzt diesen jedoch zu der $\mathrm{Pa}$ rameterzahl $P$ sowie zu der Zahl der Beobachtungen $N$ in Beziehung:

$$
A I C=\frac{-2 \ln \hat{L}\left(M_{\beta}\right)+2 P}{N} .
$$

Je mehr Parameter in dem betreffenden Modell enthalten sind, desto größer wird die errechnete Likelihood und desto kleiner wird der erste Term im Zähler. Damit die Bestimmung der Anpassung nicht allein durch zusätzlich eingefugte erklärende Variablen erfolgt, wird die Zahl der Parameter im Zähler ebenfalls berücksichtigt. Eine hohe Parameterzahl verringert c. p. die Anpassungsgüte, in-

${ }^{182}$ Akaike's Information Criterion. 
dem das AIC ansteigt, während das Modell umso besser spezifiziert ist, je kleiner das AIC ist. Eine große Zahl an verfügbaren Beobachtungen $N$ hat schließlich ebenfalls eine Verringerung des AIC zur Konsequenz.

\section{B Grundlagen der Panelschätzung - Fixed versus Random Effects}

Eine Regression mit Paneldaten unterscheidet sich von Querschnittsanalysen dadurch, dass nunmehr nicht nur die Individuen, sondern auch verschiedene Zeitpunkte erfasst werden. In seiner allgemeinen Form lässt sich ein solches Modell in Form von

$$
y_{i t}=\boldsymbol{x}_{i t} \boldsymbol{\beta}+\varepsilon_{i t}, \quad i=1, \ldots, N, t=1, \ldots, T
$$

schreiben. Der Index $i$ bezeichnet hierbei die erfassten Haushalte oder Individuen, während $t$ den Zeitindex darstellt. Somit ist $i$ Ausdruck der Querschnittsdimension, während $t$ die Längsschnittdimension widerspiegelt (vgl. Baltagi (2005), S. 11). $\boldsymbol{x}_{i t}$ kann Variablen enthalten, die sich nur zwischen den Individuen und nicht über die Zeit, nur in der Zeit und nicht zwischen den Individuen oder aber gleichermaßen in $i$ und $t$ ändern. Der Fehlerterm $\varepsilon_{i t}$ wird zusätzlich in einen individualspezifischen Effekt $u_{i}$ sowie in einem restlichen Störterm $v_{i t}$ getrennt:

$$
\varepsilon_{i t}=v_{i t}+u_{i}
$$

Der individualspezifische Effekt ist zeitunabhängig und beschreibt die unbeobachtbare Heterogenität zwischen den einzelnen Individuen. Der übrige Störterm variiert sowohl zwischen den Individuen als auch in der Zeit, so dass dieser die Bedeutung des üblichen Fehlerterms in einer Regression einnimmt. Wird nun $u_{i}$ als ein Parameter interpretiert, der für jede Querschnittsbeobachtung $i$ geschätzt werden kann, spricht man von einer Fixed Effects-Spezifikation. In diesem Fall wird für jedes Jahr und jedes Individuum ein eigener Dummy geschätzt, der die Individualspezifika abfangen soll, so dass insgesamt $(N-1)+(T-1)$ Dummyvariablen geschätzt werden müssen. ${ }^{183}$ Ein derartiges Vorgehen ist daher insbesondere bei sehr großen Stichproben problematisch (vgl. Pindyck und Rubinfeld (1998), S. 252).

${ }^{183}$ Jeweils eine Dummyvariable wird nicht mitgeschätzt, um perfekte Kollinearität zwischen den erklärenden Variablen zu vermeiden. Zudem reduziert sich die Zahl der Freiheitsgrade durch die zusätzlich enthaltenen Dummyvariablen. 
Random Effects ergeben sich dann, wenn $u_{i}$ als eine Zufallsvariable behandelt wird, die über den Fehlerterm mit abgebildet wird. Diese Unterscheidung bedeutet jedoch nicht, dass $u_{i}$ im Rahmen einer Fixed Effects-Schätzung als nicht zufällig angesehen wird. Vielmehr ist hier eine Korrelation zwischen den beobachteten erklärenden Variablen $\boldsymbol{x}_{i t}$ und dem individualspezifischen Effekt erlaubt, während für Random Effects gilt, dass die erklärenden Variablen und $u_{i}$ als nicht korreliert angenommen werden $\left(\operatorname{Cov}\left(x_{i t}, u_{i}\right)=0, t=1, \ldots, T\right)(\mathrm{vgl}$. Wooldridge (2002), S. 251f.).

Allgemein wird bei der Random Effects-Spezifikation angenommen, dass die spezifischen Charakteristika von Individuen zufällig über den Querschnitt verteilt sind. Diese Annahme gilt insbesondere dann, wenn die Stichprobe aus einer großen Population stammt. Die Konstante, die im Rahmen eines Fixed EffectsModells durch die Dummies ersetzt wird, beschreibt im Random Effects-Modell den Mittelwert der unbeobachteten Heterogenität. Insgesamt sinkt durch diese Vorgehensweise die Zahl der zu schätzenden Parameter beträchtlich, was sich insbesondere bei einer großen Zahl an Beobachtungen als wesentlicher Vorteil erweist (vgl. Greene (2003), S. 293f.).

Nachfolgend findet wiederum die Annahme einer latenten abhängigen Variablen Anwendung. Gleichung (8.39) verändert sich somit zu

$$
y_{i t}^{*}=x_{i t} \beta+\varepsilon_{i t}, \quad i=1, \ldots, N, t=1, \ldots, T .
$$

Im Fall einer ordinalskalierten Variablen mit mehreren Kategorien werden die Beobachtungen analog zu (8.30) bestimmten Schwellenwerten zugeordnet:

$$
y_{i t}=m \quad \text { für } \tau_{m-1} \leq y_{i t}^{*}<\tau_{m}, \quad m=1, \ldots, J,
$$

wobei erneut $\tau_{0}=-\infty$ und $\tau_{\mathrm{J}}=+\infty$ gilt. 


\section{Zusammenfassung und Politikimplikationen}

Eine gute Gesundheit ist nicht nur für jedes einzelne Individuum von unschätzbarem Wert, sondern auch für die Volkswirtschaft. Nur Menschen, die gesund sind, können ihr Arbeitspotential bestmöglich ausschöpfen, indem sie eine optimale Balance zwischen Arbeit, Weiterbildung und Erholung finden.

Die wachstumstheoretische Untersuchung des dritten Kapitels hat gezeigt, dass die Produktivität der Menschen, die in einem Land leben, für den wirtschaftlichen Erfolg maßgeblich ist. Zwar sind die Rahmenbedingungen, beispielsweise die geographische Lage oder das Rohstoffvorkommen, von Land zu Land unterschiedlich, weshalb einige Länder naturgemäß über einen Vorteil verfügen. Die wirtschaftliche Nutzung der Ressourcen wird aber durch menschliches Verhalten bestimmt, welches durch die Gesundheit auf eine direkte und eine indirekte Weise beeinflusst wird. Die direkte Wirkung ergibt sich aus den Produktivitätseffekten der Gesundheit, indem die zu verrichtende Arbeit schnell und korrekt ausgeführt werden kann. Die indirekte Wirkung folgt aus der Bedeutung einer guten Gesundheit für die Bildung.

Je besser die Gesundheit ist, desto weniger Zeitaufwand ist nötig, um einen bestimmten Bildungsstand zu erreichen, d. h. der Lernprozess erfolgt mit einer höheren Effektivität. Hieraus ergeben sich positive Einkommenseffekte, indem das zukünftige Einkommen mit der Bildung ansteigt. Darüber hinaus determiniert die Gesundheit das Risiko, zumindest einen Teil der Zeit krank zu verbringen, wodurch die Bildungsanstrengungen nicht ausreichend kompensiert würden. Im Rahmen einer modelltheoretischen Untersuchung wird gezeigt, dass Unsicherheit über die zukünftige Erwerbsfähigkeit, hervorgerufen durch ein positives Erkrankungsrisiko, zu geringe Bildungsinvestitionen nach sich zieht.

Mit Hilfe einer Versicherung kann zwar nicht das Risiko einer Erkrankung gesenkt werden, wohl aber die finanzielle Belastung, die hieraus entsteht. ${ }^{184}$ Durch die Glättung von Einkommen zwischen den Zuständen gesund und krank trägt die Versicherung dazu bei, dass Unsicherheit hinsichtlich der zukünftigen Erträge abgebaut und so das Vorsichtssparmotiv eingeschränkt wird, so dass sich Bildungsinvestitionen im Prinzip wieder stärker lohnen. Dabei sollte die Versicherung derart ausgestaltet sein, dass keine Verzerrungen zwischen den Einkommen aus Ersparnissen sowie aus Erwerbstätigkeit entstehen, da hierüber die optimale Zeitallokation bestimmt wird.

\footnotetext{
${ }^{184}$ Es sei denn, die Versicherung finanziert auch Vorsorgeleistungen, die das Erkrankungsrisiko mindern und sich so positiv auf die Erwerbsfähigkeit auswirkt.
} 
Die konkrete Wirkung der Versicherung auf die Bildungsentscheidung ist nun zum einen von dem Finanzierungssystem, zum anderen aber auch von der Entwicklung des Beitragssatzes bzw. der Prämie abhängig. Das aktuelle Beitragssystem der GKV wirkt sich verzerrend auf die Bildungsentscheidung der Individuen aus, indem ausschließlich das Erwerbseinkommen belastet wird, nicht aber andere Einkommensarten. Humankapital wird so gegenüber dem Realkapital benachteiligt, weshalb die Ersparnisbildung, auch im Hinblick auf die vorherrschende Unsicherheit über zukünftige finanzielle Belastungen, an Attraktivität gewinnt. Weniger verzerrend ist die gleichmäßige Besteuerung von Zinseinkünften und Lohneinkommen, da dann nur noch der Konsumverzicht der ersten Periode steuerfrei bleibt, nicht aber die Erträge, die sich hieraus ergeben. ${ }^{185}$ Ein Prämiensystem bleibt hingegen vollständig ohne Verzerrungen. Demnach wäre eine Prämienfinanzierung einer Steuerfinanzierung allgemein vorzuziehen. Bezogen auf die gesundheitspolitische Diskussion in Deutschland lässt sich aus dieser Perspektive nur eine Umstellung der Finanzierung auf ein einkommensunabhängiges Prämiensystem rechtfertigen, während eine Finanzierungsreform im Sinne der vielfach diskutierten Bürgerversicherung aufgrund der entstehenden Verzerrungen abzulehnen wäre (vgl. zu den Gesundheitsreformdiskussionen exemplarisch Wille und Albring (2005)).

Nun bleibt die Frage, wie konjunkturanfällig die einzelnen Systeme sind und wie sich langfristig die Steuersätze bzw. die Prämien entwickeln werden. Das gegenwärtige GKV-System bringt eine ausgeprägte Möglichkeit der Beitragsvermeidung mit sich, indem gegenwärtiges Einkommen über die Ersparnisse in zukünftige Perioden transferiert wird. Zudem ist ein starker Anstieg des Beitragssatzes bei sinkender Erwerbstätigkeit und einer gleichzeitig alternden Bevölkerung unumgänglich. Unter zusätzlicher Berücksichtigung des medizinischtechnischen Fortschritts reichen die Beitragssatzprognosen von $23,1 \%$ im günstigsten bis zu 39,5\% im schlechtesten Fall (vgl. Postler (2003), S. 23).

Durch eine Umstellung der Finanzierung auf ein System, das gleichzeitig die Ersparnisse berücksichtigt, würde zwar die Bemessungsgrundlage ausgeweitet, bei einem Anstieg des Beitragssatzes käme es jedoch erneut unweigerlich $\mathrm{zu}$ einem Anstieg der Lohnnebenkosten und damit zu einer zusätzlichen Belastung des Faktors Arbeit. Für ein Prämiensystem gilt hingegen, dass sich ein Anstieg in der Prämie zwar negativ auf die Bereitschaft zu mehr Bildung auswirkt, da die finanzielle Belastung im Krankheitsfall ebenfalls ansteigt und so das Vor-

${ }^{185}$ An dieser Stelle sei darauf hingewiesen, dass die Einfuhrung eines Sparerfreibetrages die Zinseinkünfte gegenüber den vollständig zu verbeitragenden Erwerbseinkommen in ihrer Attraktivität steigen lässt. Damit wäre wiederum eine Ungleichbehandlung von Human- und Realkapital zu Lasten der Bildung gegeben. 
sichtssparen stärker gefördert wird. Durch die geringere Konjunkturabhängigkeit können Prämienanstiege jedoch länger vermieden werden, so dass die individuelle Bildungsentscheidung einem größeren $\mathrm{Ma} ß$ an Sicherheit unterliegt.

Aus der Humankapitalperspektive stellen beide Alternativen eine Verbesserung zum Status quo dar. Mit dem Modell der Bürgerversicherung sowie den zahlreichen Prämienmodellen, die gegenwärtig die Diskussion um eine Reform der GKV bestimmen, würden daher die zahlreichen Verzerrungswirkungen der rein lohnbezogenen Finanzierung zumindest abgeschwächt, im Optimalfall sogar vollständig abgebaut werden. Dies gilt insbesondere für risikoorientierte Prämien, wie sie das Bayreuther Versichertenmodell vorsieht (vgl. Oberender et al. (2006)), da diese eine langfristige finanzielle Planungssicherheit gewährleisten. In einem solchen Modell sind keine Vermeidungseffekte mehr gegeben, da ein möglicher finanzieller Ausgleich an eine Belastungsgrenze gekoppelt ist, die sich auf das gesamte Einkommen bezieht. ${ }^{186}$

Insgesamt stellt die Diskussion über eine Finanzreform der gesetzlichen Krankenversicherung demnach zu stark auf die Einnahmenseite ab, ohne mögliche Fehlanreize, die sich für die Investitionen in Humankapital ergeben, zu bedenken. Darüber hinaus sollten jedoch auch Bildungs- und damit einhergehende Wachstumseffekte berücksichtigt werden, da sich mit Bildung einhergehende Einkommenssteigerungen positiv auf die Finanzierungsbasis auswirken.

Über die reinen Bildungseffekte hinaus muss es ein Ziel sein, dem Kosten treibenden medizinisch-technischen Fortschritt, der sich im Wesentlichen auf Produkt- und nicht auf Prozessinnovationen konzentriert, entgegenzuwirken, um Beitragssatzsteigerungen zu vermeiden. Hierzu muss sich entsprechend die Gesundheit der Bevölkerung verbessern, denn das Finanzierungsvolumen kann nur gesenkt werden, wenn die kurative Inanspruchnahme medizinischer Leistungen ebenfalls gesenkt werden kann. Eine Alternative hierzu wäre die Vorgehensweise der Rationierung, die jedoch aufgrund möglicher negativer Gesundheitseffekte weitgehend vermieden werden sollte. Andernfalls wäre die Gesundheit per se einkommensabhängig, woraus sich eine immer stärker werdende Chancenungleichheit ergeben würde.

${ }^{186}$ Das Bayreuther Versichertenmodell orientiert sich am erwarteten Schadensverlauf einer Person und damit an Durchschnittswerten der Versicherten. Insofern kann nicht von risikoäquivalenten Prämien gesprochen werden (vgl. Oberender et al. (2006)). In Bezug auf die Bildungsinvestitionen ist eine solche Vorgehensweise vorteilhaft, da Risikoäquivalenz erneut die langfristige Planungssicherheit einschränken und somit das Vorsorgesparen fördern würde, was wiederum zu Lasten der Bildungsinvestitionen ginge. 
Im Rahmen der Arbeit wird gezeigt, dass insbesondere das individuelle Verhalten für die Gesundheit eine große Rolle spielt. Dieses wird wiederum maßgeblich durch drei Faktoren bedingt, wie sowohl die theoretische als auch die empirische Analyse zeigt: Das gesundheitsrelevante Verhalten verbessert sich mit dem Bildungsstand, dem Haushaltseinkommen und der Arbeitszeit. Zumindest bei Männern können auch Arztbesuche zu einem gesundheitsbewussten Verhalten beitragen. Für die selbst eingeschätzte Gesundheit, welche für einen Arztbesuch entscheidend ist, sind die Ergebnisse ähnlich.

Aktuelle Zahlen belegen die immensen volkswirtschaftlichen Kosten, die durch ungesundes Verhalten der Individuen entstehen. Allein die Konsequenzen von Fettleibigkeit werden durch die WHO wie folgt beschrieben: „Adipositas führt auch zu einer beträchtlichen ökonomischen Belastung durch Produktivitätsausfälle und Einkommensverluste und ist für 2-8\% der gesamten Gesundheitsausgaben verantwortlich" (WHO / Weltgesundheitsorganisation (2005), S. 3). Diese Gesundheitsausgaben betrugen für das Jahr 2003 anteilig 11,3 Prozent des BIP, so dass hier ein enormes Einsparpotential besteht. Die direkten und indirekten Kosten des Rauchens wurden im Jahr 2000 auf umgerechnet 28,6 bis 43,5 Mrd. Euro geschätzt, wobei Erkrankungen, die durch Passivrauchen entstehen, nicht mit eingerechnet wurden (vgl. Welte et al. (2000), zit. in Sachverständigenrat für die Konzertierte Aktion im Gesundheitswesen (2001), S. 98). ${ }^{187}$

Aus diesen Überlegungen lassen sich folgende Politikimplikationen ableiten: Mit Hilfe einer verständlichen Aufklärung über die Folgen von Übergewicht und Tabakkonsum können Verhaltensunterschiede, die sich aus unterschiedlichen Bildungsabschlüssen ergeben, zumindest teilweise abgebaut werden. Auf diese Weise kann auch die Eigenverantwortung gestärkt werden. Ebenso von Bedeutung ist das Haushaltseinkommen, da hierüber beispielsweise der Konsum von Nahrungsmitteln und von privat $\mathrm{zu}$ finanzierenden Gesundheitsleistungen determiniert wird. Um eine Steigerung des Einkommens zu erreichen, ist es folglich wichtig, die Erwerbstätigkeit von Personen im erwerbsfähigen Alter zu fördern. Nicht zuletzt aufgrund positiver Arbeitszeiteffekte, über die die Opportunitătskosten im Krankheitsfall, ein gesteigertes Verantwortungsgefühl sowie soziale Kontakte bestimmt werden, ist eine positive Entwicklung des Arbeitsmarktes für dauerhaftes Wirtschaftswachstum ebenso wie für das Wachstum des Gesundheitssektors unabdingbar.

${ }^{187}$ Die direkten Kosten umfassen die Behandlungskosten, die unmittelbar auf Tabakkonsum zurückzufuhren sind. Mit Hilfe der indirekten Kosten wird der krankheitsbedingte Arbeitsausfall, der durch das Rauchen entsteht, bewertet. 
Gerade die Einkommensabhängigkeit des Gesundheitsverhaltens zeigt zudem, dass Maßnahmen, die die Eigenverantwortung stärken sollen, derart ausgestaltet sein müssen, dass insbesondere sozial Schwache nicht durch das System benachteiligt werden. Es muss daher sichergestellt sein, dass Prävention nicht ebenfalls eine Frage des Einkommens ist. Zudem sollten die Konsequenzen ungesunden Verhaltens deutlich aufgezeigt und vor allem Lösungsansätze vermittelt werden, die selbst für Personen mit niedriger Bildung und geringem Einkommen nachvollziehbar und damit auch durchführbar sind. 


\section{Literaturverzeichnis}

Aghion, P. und P. Howitt (1999), Endogenous Growth Theory, 3 Aufl., Cambridge, MA: MIT Press.

Aitchison, J. und J. Brown (1976), The Lognormal Distribution with Special Reference to its Uses in Economics, Cambridge et al.: Cambridge University Press.

Alderman, H., Behrman, J. R., Lavy, V. und R. Menon (2001), Child Health and School Enrollment - A Longitudinal Analysis, in: Journal of Human Resources, 36, 185-205.

Amemiya, T. (1981), Qualitative Response Models: A Survey, in: Journal of Economic Literature, 19, 1483-1536.

Anderberg, D. und F. Andersson (2000), Social Insurance with Risk-Reducing Investments, in: Economica, 67, 37-56.

Andrén, D. und E. Palmer (2001), The Effect of Sickness on Earnings, Working Papers in Economics Nr. 45, Göteborg University, Göteborg.

Arora, S. (2001), Health, Human Productivity, and Long-Term Economic Growth, in: Journal of Economic History, 61, 699-749.

Arrow, K. J. (1976), The Theory of Risk Aversion, in: Arrow, K. J. (Hg.), Essays in the Theory of Risk-Bearing, Amsterdam und Oxford: North-Holland, 90-120.

Baltagi, B. H. (2005), Econometric Analysis of Panel Data, 3. Aufl., Chichester: Wiley.

Barro, R. J. (1997), Determinants of Economic Growth: A Cross-country Empirical Study, Cambridge und London: MIT Press.

Barro, R. J. (2001), Human Capital: Growth, History and Policy - A Session to Honor Stanley Engerman, in: American Economic Review, 91, 12-17.

Barro, R. J. und J. Lee (2000), International Data on Educational Attainment Updates and Implications, NBER Working Paper Nr. 7911, National Bureau of Economic Research, Cambridge, MA. 
Barro, R. J. und X. Sala-i-Martin (1995), Economic Growth, New York et al.: McGraw-Hill.

Barro, R. J. und X. Sala-i-Martin (1997), Technological Diffusion, Convergence, and Growth, in: Journal of Economic Growth, 2, 1-27.

Barro, R. J. und X. Sala-i-Martin (2003), Economic Growth, 2 Aufl., Cambridge, MA: The MIT Press.

Becker, G. S. (1965), A Theory of the Allocation of Time, in: The Economic Journal, 75, 493-517.

Becker, G. S. (1993), Human capital: A Theoretical and Empirical Analysis, with special Reference to Education, 3. Aufl., Chicago und London: University of Chicago Press.

Behrman, J. R. (1996), The Impact of Health and Nutrition on Education, in: The World Bank Research Observer, 11, 23-38.

Ben-Porath, Y. (1967), The Production of Human Capital and the Life Cycle of Earnings, in: Journal of Political Economy, 75, 352-365.

Benhabib, J. und M. M. Spiegel (1994), The Role of Human Capital in Economic Development. Evidence from Aggregate Cross-Country Data, in: Journal of Monetary Economics, 34, 143-173.

Berger, M. C. und J. P. Leigh (2001), Schooling, Self-Selection, and Health, in: Journal of Human Resources, 24, 433-455.

Berkowitz, M., Fenn, P. und J. Lambrinos (1983), The Optimal Stock of Health with Endogenous Wages, in: Journal of Health Economics, 2, 139-147.

Bernanke, B. S. und R. S. Gürkaynak (2001), Is Growth Exogenous? Taking Mankiw, Romer, and Weil Seriously, in: NBER Macroeconomics Annual, 16, 11-57.

Bhargava, A., Jamison, D. T., Lau, L. J. und C. J. L. Murray (2001), Modeling the Effects of Health on Economic Growth, in: Journal of Health Economics, $20,423-440$.

Bhattacharya, J., Currie, J. und S. Haider (2004), Poverty, Food Insecurity, and Nutritional Outcomes in Children and Adults, in: Journal of Health Economics, 23, 839-862. 
Bils, M. und P. J. Klenow (2000), Does Schooling Cause Growth? in: American Economic Review, 90, 1160-1183.

Blackburn, K., Hung, V. T. Y. und A. F. Pozzolo (2000), Research, Development and Human Capital Accumulation, in: Journal of Macroeconomics, 22, 189-206.

Blinder, A. S. und Y. Weiss (1976), Human Capital and Labor Supply: A Synthesis, in: Journal of Political Economy, 84, 449-472.

Bloom, D. E., Canning, D. und J. Sevilla (2004), The Effect of Health on Economic Growth: A Production Function Approach, in: World Development, $32,1-13$.

Bodenhöfer, H. und M. Riedel (1998), Bildung und Wirtschaftswachstum. Alte und neue Ansätze, in: Weizsäcker, R. K. v. (Hg.), Bildung und Wirtschaftswachstum, Schriften des Vereins für Socialpolitik, Neue Folge, Bd. 258, Berlin: Duncker \& Humblot, 11-47.

Breyer, F., Zweifel, P. und M. Kifmann (2005), Gesundheitsökonomik, 5. Aufl., Berlin et al.: Springer.

Brown, E. und H. Kaufold (1988), Human Capital Accumulation and the Optimal Level of Unemployment Insurance Provision, in: Journal of Labor Economics, 6, 493-514.

Brunello, G. (2002), Absolute Risk Aversion and the Returns to Education, in: Economics of Education Review, 21, 635-640.

Bundesministerium für Familie, Senioren, Frauen und Jugend (2004), Bericht über die Auswirkungen der $\S \S 15$ und 16 Bundeserziehungsgeldgesetz des Bundesministeriums für Familie, Senioren, Frauen und Jugend, Berlin.

Bundesministerium für Gesundheit und Soziale Sicherung (2003), Nachhaltigkeit in der Finanzierung der Sozialen Sicherungssysteme, Bericht der Kommission, Berlin.

Bundesministerium für Gesundheit und Soziale Sicherung (2005), Statistisches Taschenbuch Gesundheit 2005, Berlin.

Case, A., Fertig, A. und C. Paxson (2003), From Cradle to Grave? The Lasting Impact of Childhood Health and Circumstance, National Bureau of Econo- 
mic Research (Hg.), NBER Working Paper Series Nr. 9788, Cambridge, MA.

Case, A., Fertig, A. und C. Paxson (2005), The Lasting Impact of Childhood Health and Circumstance, in: Journal of Health Economics, 24, 365-389.

Chakraborty, S. (2003), Endogenous Lifetime and Economic Growth, Working Paper Nr. 23, Department of Economics, University of Oregon.

Chakraborty, S. (2004), Endogenous Lifetime and Economic Growth, in: Journal of Economic Theory, 116, 119-137.

Chirikos, T. N. und G. Nestel (1985), Further Evidence on the Economic Effects of Poor Health, in: The Review of Economics and Statistics, 67, 61-69.

Cremieux, P. Y., Ouellette, P. und C. Pilon (1999), Health Care Spending as Determinants of Health Outcomes, in: Health Economics, 8, 627-639.

Cropper, M. L. (1977), Health, Investment in Health, and Occupational Choice, in: Journal of Political Economy, 85, 1273-1294.

Currie, J. und B. C. Madrian (1999), Health, Health Insurance and the Labor Market, in: Ashenfelter, O. und D. Card (Hg.), Handbook of Labor Economics, Bd. 3C, Amsterdam et al.: Elsevier, 3309-3415.

Currie, J. und M. Stabile (2003), Socioeconomic Status and Child Health: Why is the Relationship Stronger for Older Children? in: American Economic Review, 93, 1813-1823.

Currie, J. und R. Hyson (1999), Is the Impact of Health Shocks Cushioned by Socioeconomic Status? The Case of Low Birthweight, in: American Economic Review, 89, 245-250.

Dardanoni, V. und A. Wagstaff (1990), Uncertainty and the Demand for Medical Care, in: Journal of Health Economics, 9, 23-38.

Dinopoulos, E. und P. Thompson (1996), A Contribution to the Empirics of Endogenous Growth, in: Eastern Economic Journal, 22, 389-400.

De la Croix, D. und O. Licandro (1999), Life Expectancy and Endogenous Growth, in: Economics Letters, 65, 255-263.

Dolan, P. (2000), The Measurement of Health-Related Quality of Life for Use in Resource Allocation Decisions in Health Care, in: Culyer, A. J. und J. P. 
Newhouse (Hg.), Handbook of Health Economics, Bd. 1B, Amsterdam et al.: Elsevier, 1723-1760.

Duraisamy, P. und D. Sathiyavan (1998), Impact of Health Status on Wages and Labour Supply of Men and Women, in: Indian Journal of Labour Economics, 41, 67-84.

Edwards, L. N. und M. Grossman (1979), The Relationship Between Children's Health and Intellectual Development, in: Mushkin, S. J. und D. W. Dunlop (Hg.), Health: What Is It Worth? Measures of Health Benefits, New York et al.: Pergamon Press, 273-314.

Ehrlich, I. und G. S. Becker (1972), Market Insurance, Self-Insurance, and SelfProtection, in: Journal of Political Economy, 80, 623-648.

Ehrlich, I. und H. Chuma (1990), A Model of the Demand for Longevity and the Value of Life Extension, in: Journal of Political Economy, 98, 761-782.

Eisenring, C. (2000), Is There a Trade-Off between Longevity and Quality of Life in Grossman's Pure Investment Model? in: Health Economics, 9, 669680 .

Erbsland, M., Ried, W. und V. Ulrich (1998), The Impact of the Environment on the Demands for Health and Health Care: An Empirical Analysis for Germany, in: Zweifel, P. (Hg.), Health, the Medical Profession, and Regulation, Dordrecht: Kluwer, 3-34.

Ettner, S. L. (1996), New Evidence on the Relationship between Income and Health, in: Journal of Health Economics, 15, 67-85.

Ettner, S. L. (2000), The Relationship Between Labor Market Outcomes and Physical and Mental Health, in: Salkever, D. und A. Sorkin (Hg.), The Economics of Disability, Stanford: Stanford University, 1-31.

Fogel, R. F. (1994), Economic Growth, Population Theory, and Physiology: The Bearing of Long-Term Processes on the Making of Economic Policy, in: American Economic Review, 84, 369-395.

Foster, A. D. (1994), Poverty and Illness in Low-Income Rural Areas, in: American Economic Review, 84, 216-220. 
French, E. (2000), The Effects of Health, Wealth, and Wages on Labor Supply and Retirement Behavior, Federal Reserve Bank of Chicago (Hg.), Working Papers Series Nr. WP 200-02, Chicago.

Galor, O. und D. Tsiddon (1997), The Distribution of Human Capital and Economic Growth, in: Journal of Economic Growth, 2, 93-124.

Gemmell, N. (1995), Endogenous Growth, the Solow Model and Human Capital, in: Economics of Planning, 28, 169-183.

Gerdtham, U. und M. Johannesson (1999), New Estimates of the Demand for Health: Results based on a Categorical Health Measure and Swedish Micro Data, in: Social Science \& Medicine, 49, 1325-1332.

Gersovitz, M. (1983), Savings and Nutrition at Low Incomes, in: Journal of Political Economy, 91, 841-855.

Gilleskie, D. B. (1998), A Dynamic Stochastic Model of Medical Care Use and Work Absence, in: Econometrica, 66, 1-45.

Gilleskie, D. B. und A. L. Harrison (1998), The Effect of Endogenous Health Inputs on the Relationship between Health and Education, in: Economics of Education Review, 17, 279-297.

Glewwe, P. (2002), Schools and Skills in Developing Countries: Education Policies and Socioeconomic Outcomes, in: Journal of Economic Literature, 40, 436-482.

Goetz, S. J. und D. Hu (1996), Economic Growth and Human Capital Accumulation: Simultaneity and Expanded Convergence Tests, in: Economics Letters, 51, 355-362.

Goodman, A. C., Stano, M. und J. M. Tilford (1999), Household Production of Health Investment: Analysis and Applications, in: Southern Economic Journal, 65, 791-806.

Greene, W. H. (2003), Econometric Analysis, 5. Aufl., Upper Saddle River: Pearson Education.

Grossman, G. M. und E. Helpman (1994), Endogenous Innovation in the Theory of Growth, in: Journal of Economic Perspectives, 8, 23-44. 
Grossman, M. (1970), The Demand for Health: A Theoretical and Empirical Investigation, Columbia University: Dissertation.

Grossman, M. (1972), On the Concept of Health Capital and the Demand for Health, in: Journal of Political Economy, 80, 223-255.

Grossman, M. (1975), The Correlation between Health and Schooling, in: Terleckyj, N. E. (Hg.), Household Production and Consumption, New York: Columbia University Press, 147-223.

Grossman, M. (2000), The Human Capital Model, in: Culyer, A. J. und J. P. Newhouse (Hg.), Handbook of Health Economics, Bd. 1A, Amsterdam et al.: Elsevier, 347-408.

Grossman, M. und L. Benham (1974), Health, Hours and Wages, in: Perlman, M. (Hg.), The Economics of Health and Medical Care, New York: Halsted Press, 206-233.

Gyimah-Brempong, K. und M. Wilson (2004), Health Human Capital and Economic Growth in Sub-Saharan African and OECD Countries, in: The Quarterly Review of Economics and Finance, 44, 296-320.

Haveman, R., Wolfe, B. L., Kreider, B. und M. Stone (1994), Market Work, Wages, and Men's Health, in: Journal of Health Economics, 13, 163-182.

Henke, K., Borchardt, K., Schreyögg, J. und O. Farhauer (2003), Eine ökonomische Analyse unterschiedlicher Finanzierungsmodelle der Krankenversorgung in Deutschland, Wirtschaftswissenschaftliche Dokumentation der TU Berlin (Hg.), Diskussionspapier Nr. 2003/4, Berlin.

Henke, K., Martin, K. und C. S. Behrens (1997), Direkte und indirekte Kosten von Krankheiten in der Bundesrepublik Deutschland 1980 und 1990, in: Zeitschrift für Gesundheitswissenschaften, 5, 123-145.

Hernández-Quevedo, C., Jones, A. M. und N. Rice (2004), Reporting Bias and Heterogeneity in Self-Assessed Health. Evidence from the British Household Panel Survey, Discussion Papers in Economics Nr. 2004/18, Department of Economics and Related Studies, University of York, Heslington, York.

Howitt, P. (2005), Health, Human Capital, and Economic Growth: A Schumpeterian Perspective, in: López-Casanovas, G., Rivera, B. und L. Currais (Hg.), Health and Economic Growth. Findings and Policy Implications, Cambridge: MIT Press, 19-40. 
Jamison, D. T., Lau, L. J. und J. Wang (2005), Health's Contribution to Economic Growth in an Environment of Partially Endogenous Technical Progress, in: López-Casanovas, G., Rivera, B. und L. Currais (Hg.), Health and Economic Growth. Findings and Policy Implications, Cambridge: MIT Press, 67-91.

Johansson, P. und K. Löfgren (1995), Wealth from Optimal Health, in: Journal of Health Economics, 14, 65-79.

Johnson, N. L., Kotz, S. und N. Balakrishnan (1994), Continuous Univariate Distributios, Bd. 1, 2. Aufl., New York et al.: Wiley-Interscience.

Kalemli-Ozcan, S. (2002), Does the Mortality Decline Promote Economic Growth? in: Journal of Economic Growth, 7, 411-439.

Kalemli-Ozcan, S. (2003), A Stochastic Model of Mortality, Fertility, and Human Capital Investment, in: Journal of Development Economics, 70, 103118.

Kalemli-Ozcan, S., Ryder, H. E. und D. N. Weil (2000), Mortality Decline, Human Capital Investment, and Economic Growth, in: Journal of Development Economics, 62, 1-23.

Kemna, H. J. M. I. (1987), Working Conditions and the Relationship between Schooling and Health, in: Journal of Health Economics, 6, 189-210.

Kenkel, D. S. (1991), Health Behavior, Health Knowledge, and Schooling, in: Journal of Political Economy, 99, 286-305.

Kennedy, P. (2003), A Guide to Econometrics, 5 Aufl., Cambridge, MA: MIT Press.

Khan, Q. M. (1984), The Impact of Household Endowment Constraints on Nutrition and Health, in: Journal of Development Economics, 15, 313-328.

Knowles, S. und P. D. Owen (1995), Health Capital and Cross-Country Variation in Income per Capita in the Mankiw-Romer-Weil Model, in: Economics Letters, 48, 99-106.

Knowles, S. und P. D. Owen (1997), Education and Health in an Effective-Labour Empirical Growth Model, in: Economic Record, 73, 314-328. 
Kodde, D. A. (1986), Uncertainty and the Demand for Education, in: Review of Economics and Statistics, 68, 460-467.

Krebs, T. (2003), Human Capital Risk and Economic Growth, in: Quarterly Journal of Economics, 118, 709-744.

Kruse, A., Knappe, E., Schulz-Nieswandt, F., Schwartz, F. und J. Wilbers (2003), Kostenentwicklung im Gesundheitswesen: Verursachen ältere Menschen höhere Gesundheitskosten? Expertise, erstellt im Auftrag der AOK Baden-Württemberg, Heidelberg.

Lambrinos, J. (1981), Health: A Source of Bias in Labor Supply Models, in: Review of Economics and Statistics, 63, 206-212.

Lee, L. (1982), Health and Wage: A Simultaneous Equation Model with Multiple Discrete Indicators, in: International Economic Review, 23, 199-221.

Leibowitz, A. A. (2004), The Demand for Health and Health Concerns after 30 Years, in: Journal of Health Economics, 23, 663-671.

Leigh, J. P. (1983), Direct and Indirect Effects of Education on Health, in: Social Science and Medicine, 17, 227-234.

Leontaridi, R. M. und M. E. Ward (2002), Work-Related Stress, Quitting Intentions and Absenteeism, IZA Bonn (Hg.), IZA Discussion Paper Nr. 493, Bonn.

Leu, R. E. und M. Gerfin (1992), Die Nachfrage nach Gesundheit - Ein empirischer Test des Grossmann-Modells, in: Oberender, P. (Hg.), Steuerungsprobleme im Gesundheitswesen, Gesundheitsökonomische Beiträge, Band 14, Baden Baden: Nomos, 61-79.

Leu, R. E. und R. J. Doppmann (1986), Die Nachfrage nach Gesundheit und Gesundheitsleistungen, in: Gäfgen, G. (Hg.), Okonomie des Gesundheitswesens, Schriften des Vereins für Socialpolitik; N. F., Bd. 159, Berlin: Duncker \& Humblot, 161-175.

Leu, R. E., Doppmann, R., Keller, T. und R. Deutschmann (1986), Die quantitative Erfassung von Gesundheitszustand und Lebensqualität, illustriert am Beispiel von Psoriasiskranken, in: Horisberger, B. und W. van Eimeren (Hg.), Die Kosten-Nutzen-Analyse. Methodik und Anwendung am Beispiel von Medikamenten, Berlin et al.: Springer, 155-248. 
Levhari, D. und Y. Weiss (1974), The Effect of Risk on the Investment in Human Capital, in: American Economic Review, 64, 950-963.

Liljas, B. (1998), The Demand for Health with Uncertainty and Insurance, in: Journal of Health Economics, 17, 153-170.

Long, J. S. (1997), Regression Models for Categorical and Limited Dependent Variables, Thousand Oaks et al.: Sage Publications.

Long, J. S. und J. Freese (2003), Regression Models for Categorical Dependent Variables Using Stata, College Station: Stata Press.

Lucas, R. E. (1988), On the Mechanics of Economic Development, in: Journal of Monetary Economics, 22, 3-42.

Maddala, G. S. (1999), Limited Dependent and Qualitative Variables in Econometrics, Cambridge: Cambridge University Press.

Mankiw, N. G., Romer, D. und D. N. Weil (1992), A Contribution to the Empirics of Economic Growth, in: Quarterly Journal of Economics, 107, 407437.

Mathers, C. (1994), Health Differentials among Adult Australians aged 25-64 Years, Canberra: AGPS.

Mayer-Foulkes, D. (2005), Human Development Traps and Economic Growth, in: López-Casanovas, G., Rivera, B. und L. Currais (Hg.), Health and Economic Growth. Findings and Policy Implications, Cambridge: MIT Press, 115-141.

McGuire, A., Henderson, J. und G. Mooney (1997), The Economics of Health Care. An Introductory Text, London et al.: Routledge.

McKelvey, R. D. und W. Zavoina (1975), A Statistical Model for the Analysis of Ordinal Level Dependent Variables, in: Journal of Mathematical Socio$\log y, 4,103-120$.

Mincer, J. (1958), Investment in Human Capital and Personal Income Distribution, in: Journal of Political Economy, 66, 281-302.

Mincer, J. (1984), Human Capital and Economic Growth, in: Economics of Education Review, 3, 195-205. 
Mushkin, S. J. (1962), Health as an Investment, in: Journal of Political Economy, 70, S129-S157.

Muurinen, J. (1982), Demand for Health, in: Journal of Health Economics, 1, 528.

Muysken, J., Yetkiner, I. H. und T. Ziesemer (2003), Health, Labour Productivity and Growth, in: Hagemann, H. und S. Seiter (Hg.), Growth Theory and Growth Policy, London: Routledge, 187-205.

National Research Council (1989), Diet and Health. Implications for Reducing Chronic Disease Risk, Washington D.C.: National Academy Press.

Nelson, R. R. und E. S. Phelps (1966), Investment in Humans, Technological Diffusion, and Economic Growth, in: American Economic Review, 56, 6975.

Nishiyama, K. und J. V. Johnson (1997), Karoshi - Death from Overwork: Occupational Health Consequences of Japanese Production Management, in: International Journal of Health Services, 27, 625-641.

Nocera, S. und P. Zweifel (1998), The Demand for Health: An Empirical Test of the Grossman Model Using Panel Data, in: Zweifel, P. (Hg.), Health, the Medical Profession, and Regulation, Dordrecht: Kluwer, 35-49.

Oberender, P. O., Felder, S., Ulrich, V., Schneider, U., Werblow, A. und J. Zerth (2006), Bayreuther Versichertenmodell. Der Weg in ein freiheitliches Gesundheitswesen, Bayreuth: Verlag P. C. O.

Oberender, P., Hebborn, A. und J. Zerth (2002), Wachstumsmarkt Gesundheit, Stuttgart: Lucius \& Lucius.

Over, M., Ellis, R. P., Huber, J. H. und O. Solon (1992), The Consequences of Adult Ill-Health, in: International Bank for Reconstruction and Development (Hg.), The Health of Adults in the Developing World, New York et al.: Oxford University Press, 161-208.

Papageorgiou, C. (2003), Distinguishing Between the Effects of Primary and Post-primary Education on Economic Growth, in: Review of Development Economics, 7, 622-635. 
Pauly, M. V., Nicholson, S., Xu, J., Polsky, D., Danzon, P. M., Murray, J. F. und M. L. Berger (2002), A General Model of the Impact of Absenteeism on Employers and Employees, in: Health Economics, 11, 221-231.

Perri, T. J. (1984), Health Status and Schooling Decisions of Young Men, in: Economics of Education Review, 3, 207-213.

Pindyck, R. S. und D. L. Rubinfeld (1998), Econometric Models and Economic Forecasts, 4. Aufl., Boston, Mass. et al.: McGraw-Hill.

Pitt, M. M., Rosenzweig, M. R. und M. N. Hassan (1990), Productivity, Health, and Inequality in the Intrahousehold Distribution of Food in Low-Income Countries, in: American Economic Review, 80, 1139-1156.

Postler, A. (2003), Modellrechnungen zur Beitragssatzentwicklung in der Gesetzlichen Krankenversicherung. Auswirkungen von demographischem Wandel und medizinisch-technischem Fortschritt, Diskussionsbeiträge der Fakultät für Wirtschaftswissenschaft der Universität Duisburg-Essen Nr. 298, Universität Duisburg-Essen, Duisburg.

Pritchett, L. und L. H. Summers (1996), Wealthier is Healthier, in: Journal of Human Resources, 31, 841-868.

Psacharopoulos, G. (1982), Education as an Investment, in: Finance and Development, 19, 39-42.

Ram, R. und T. W. Schultz (1979), Life Span, Health, Savings, and Productivity, in: Economic Development and Cultural Change, 27, 399-421.

Rebelo, S. (1991), Long-Run Policy Analysis and Long-Run Growth, in: Journal of Political Economy, 99, 500-521.

Ried, W. (1998a), QALYs versus HYEs - What's Right and What's Wrong. A Review of the Controversy, in: Journal of Health Economics, 17, 607-25.

Ried, W. (1998b), Comparative Dynamic Analysis of the Full Grossman Model, in: Journal of Health Economics, 17, 383-425.

Rillaers, A. (2001), Education and Income Inequality: The Role of a Social Protection System, in: Journal of Population Economics, 14, 425-443.

Rivera, B. und L. Currais (1999), Economic Growth and Health: Direct Impact or Reverse Causation? in: Applied Economics Letters, 6, 761-764. 
Rivera, B. und L. Currais (2000), The Contribution of Publicly Provided Health to Growth and Productivity, in: Estudos Economicos, 30, 191-206.

Rivera, B. und L. Currais (2004), Public Health Capital and Productivity in the Spanish Regions: A Dynamic Panel Data Model, in: World Development, 32, 871-885.

Romer, P. M. (1986), Increasing Returns and Long-Run Growth, in: Journal of Political Economy, 94, 1002-1037.

Romer, P. M. (1990), Endogenous Technological Change, in: Journal of Political Economy, 98, S71-S102.

Rosen (1976), A Theory of Life Earnings, in: Journal of Political Economy, 84, S45-S66.

Rostow, W. W. (1990), The Stages of Economic Growth: A Non-communist Manifesto, 3. Aufl., Cambridge: Cambridge University Press.

Rürup, B. und E. Wille (2004), Finanzierungsreform in der Krankenversicherung. Gutachten vom 15. Juli 2004.

Sachverständigenrat für die Konzertierte Aktion im Gesundheitswesen (1994), Gesundheitsversorgung und Krankenversicherung 2000: Eigenverantwortung, Subsidiarität und Solidarität bei sich ändernden Rahmenbedingungen, Sachstandsbericht 1994, Baden-Baden: Nomos.

Sachverständigenrat für die Konzertierte Aktion im Gesundheitswesen (1996), Gesundheitswesen in Deutschland: Kostenfaktor und Zukunftsbranche, Band I: Demographie, Morbidität, Wirtschaftlichkeitsreserven und Beschäftigung, Sondergutachten 1996, Baden-Baden: Nomos.

Sachverständigenrat für die Konzertierte Aktion im Gesundheitswesen (2001), Bedarfsgerechtigkeit und Wirtschaftlichkeit, Band III: Über-, Unter- und Fehlversorgung, Baden-Baden: Nomos.

Sachverständigenrat für die Konzertierte Aktion im Gesundheitswesen (2003), Finanzierung, Nutzerorientierung und Qualität, Band I: Finanzierung und Nutzerorientierung, Baden-Baden: Nomos.

Sachverständigenrat zur Begutachtung der gesamtwirtschaftlichen Entwicklung (2003), Staatsfinanzen konsolidieren - Steuersystem reformieren, Jahresgutachten 2003/04, Stuttgart: Metzler-Poeschel. 
Sala-i-Martin, X. (2005), On the Health-Poverty Trap, in: López-Casanovas, G., Rivera, B. und L. Currais (Hg.), Health and Economic Growth. Findings and Policy Implications, Cambridge: MIT Press, 95-114.

Schneider, U. (2002), Theorie und Empirie der Arzt-Patient-Beziehung: Zur Anwendung der Principal-Agent-Theorie auf die Gesundheitsnachfrage, Frankfurt a. M.: Peter Lang.

Schofield, D. (1996), The Impact of Employment and Hours of Work on Health Status and Health Service Use, Discussion Paper Nr. 11, NATSEM, Universtiy of Canberra, Canberra.

Schultz, T. P. (1997), Assessing the Productive Benefits of Nutrition and Health: An Integrated Human Capital Approach, in: Journal of Econometrics, 77, 141-158.

Schultz, T. P. (1999), Health and Schooling Investments in Africa, in: Journal of Economic Perspectives, 13, 67-88.

Schultz, T. W. (1961), Investment in Human Capital, in: American Economic Review, 51, 1-17.

Schäper, C. (2003), Growth and Distribution Effects of Education Policy in an Endogenous Growth Model with Human Capital Accumulation, in: Hagemann, H. und S. Seiter (Hg.), Growth Theory and Growth Policy, London: Routledge, 136-155.

Selden, T. M. (1993), Uncertainty and Health Care Spending by the Poor: The Health Capital Model revisited, in: Journal of Health Economics, 12, 109115.

Shakotko, R. A., Edwards, L. N. und M. Grossman (1981), An Exploration of the Dynamic Relationship between Health and Cognitive Development in Adolescence, in: Gaag, J. v. d. und M. Perlman (Hg.), Health, Economics, and Health Economics, Amsterdam et al.: North-Holland, 305-325.

Shields, M. (2000), Long Working Hours and Health, in: Perspectives in Labour and Income, 12, 49-56.

Smith, J. P. (1999), Healthy Bodies and Thick Wallets: The Dual Relation between Health and Economic Status, in: Journal of Economic Perspectives, $13,145-166$. 
Snow, A. und R. S. J. Warren (1990), Human Capital Investment and Labor Supply under Uncertainty, in: International Economic Review, 31, 195-206.

Solow, R. M. (1956), A Contribution to the Theory of Economic Growth, in: Quarterly Journal of Economics, 70, 65-94.

Solow, R. M. (2000), Growth Theory: An Exposition, 2 Aufl., New York: Oxford University Press.

Sparks, K., Cooper, C., Fried, Y. und A. Shirom (1997), The Effects of Hours of Work on Health: A Meta-Analytic Review, in: Journal of Occupational and Organizational Psychology, 70, 391-408.

Spurgeon, A., Harrington, J. M. und C. L. Cooper (1997), Health and Safety Problems Associated with Long Working Hours: A Review of the Current Position, in: Occupational and Environmental Medicine, 54, 367-375.

Statistisches Bundesamt (2003), Bevölkerung Deutschlands bis 2050 - 10. koordinierte Bevölkerungsvorausberechnung, Wiesbaden.

Stewart, J. M. (2001), The Impact of Health Status on the Duration of Unemployment Spells and the Implications for Studies of the Impact of Unemployment on Health Status, in: Journal of Health Economics, 20, 781796.

Strauss, J. und D. Thomas (1998), Health, Nutrition, and Economic Development, in: Journal of Economic Literature, 36, 766-817.

Sundberg, G. (1996), Essays on Health Economics, Economic Studies Nr. 26, Department of Economics, Uppsala University, Uppsala.

Sundberg, G. (1998), Health, Work Hours, and Wages in Sweden, in: Zweifel, P. (Hg.), Health, the Medical Profession, and Regulation, Boston et al.: Kluwer, 95-111.

Swanson, C. E. und K. J. Kopecky (1999), Lifespan and Output, in: Economic Inquiry, 37, 213-235.

Swanson, C. E. und K. J. Kopecky (2000), Health, Lifespan and Economic Activity: Why Poor Nations Remain Poor and Rich Nations Rich, in: Global Business \& Economics Review, 2, 185-200. 
Todaro, M. P. und S. C. Smith (2003), Economic Development, 8. Aufl., Boston et al.: Pearson Addison Wesley.

Ulrich, V. (2001), Demographische Alterung und medizinischer Fortschritt Mehr als ein potentieller Sprengsatz für die GKV? in: Schmähl, W. und V. Ulrich (Hg.), Soziale Sicherung und demographische Herausforderungen, Tübingen: Mohr Siebeck, 23-43.

Uzawa, H. (1965), Optimum Technical Change in an Aggregative Model of Economic Growth, in: International Economic Review, 6, 18-31.

Van Zon, A. und J. Muysken (2001), Health and Endogenous Growth, in: Journal of Health Economics, 20, 169-185.

Van Zon, A. und J. Muysken (2005), Health as a Principal Determinant of Economic Growth, in: López-Casanovas, G., Rivera, B. und L. Currais (Hg.), Health and Economic Growth. Findings and Policy Implications, Cambridge: MIT Press, 41-65.

Veall, M. R. und K. F. Zimmermann (1992), Pseudo-R's in the Ordinal Probit Model, in: Journal of Mathematical Sociology, 16, 333-342.

Veall, M. R. und K. F. Zimmermann (1996), Pseudo-R ${ }^{2}$ Measures for Some Common Limited Dependent Variable Models, in: Journal of Economic Surveys, 10, 241-259.

WHO / Weltgesundheitsorganisation (2005), Adipositas: Eine Herausforderung für die europäische Region der WHO, Faktenblatt Euro/13/05.

WHO / World Health Organization (2003), Diet, Nutrition and the Prevention of Chronic Diseases. Report of a Joint WHO/FAO Expert Consultation, WHO Technical Report Series Nr. 916, Geneva.

Wagstaff, A. (1986), The Demand for Health: Some New Empirical Evidence, in: Journal of Health Economics, 5, 195-233.

Wasem, J. und S. Greß (2004), Geschlechtsspezifische Auswirkungen von Leistungsausgrenzungen und Zuzahlungserhöhungen in der Gesetzlichen Krankenversicherung, Kurzexpertise für das Wirtschafts- und Sozialwissenschaftliche Institut (WSI) der Hans-Böckler-Stiftung.

Webber, D. J. (2002), Policies to Stimulate Growth: Should we Invest in Health or Education? in: Applied Economics, 34, 1633-1643. 
Weil, D. N. (2005), Accounting for the Effect of Health on Economic Growth, Department of Economics, Brown University, Providence.

Welch, F. (1970), Education in Production, in: Journal of Political Economy, $78,35-59$.

Welte, R., König, H. und R. Leidl (2000), The Costs of Health Damage and Productivity Losses attributable to Cigarette Smoking in Germany, in: European Journal of Public Health, 40, 31-44.

Wille, E. und M. Albring (2005), Versorgungsstrukturen und Finanzierungsoptionen auf dem Prüfstand, Frankfurt a. M.: Peter Lang.

Williams, A. (1985), The Nature, Meaning and Measurement of Health and Illness: An Economic Viewpoint, in: Social Science \& Medicine, 20, 10231027.

Williams, J. T. (1979), Uncertainty and the Accumulation of Human Capital over the Life Cycle, in: Journal of Business, 52, 521-548.

Wolfe, B. L. (1985), The Influence of Health on School Outcomes, in: Medical Care, 23, 1127-1138.

Wooldridge, J. M. (2002), Econometric Analysis of Cross Section and Panel Data, Cambridge, Mass.: MIT Press.

Wooldridge, J. M. (2003), Introductory Econometrics: A Modern Approach, Mason: South-Western.

World Bank (1993), World Development Report: Investing in Health, Oxford: Oxford University Press.

Yaari, M. E. (1969), Uncertain Lifetime, Life Insurance, and the Theory of the Consumer, in: Review of Economic Studies, 32, 137-150.

Zdrowomyslaw, N. und W. Dürig (1997), Gesundheitsökonomie, München: Oldenbourg.

Zhang, J. (2003), Optimal Dept, Endogenous Fertility, and Human Capital Externalities in a Model with Altruistic Bequests, in: Journal of Public Economics, 87, 1825-1835.

Zhang, J., Zhang, J. und R. Lee (2003), Rising Longevity, Education, Savings, and Growth, in: Journal of Development Economics, 70, 83-101. 
Zweifel, P. und G. Zysset-Pedroni (1992), Was ist Gesundheit und wie läßt sie sich messen? Bd. 1: Einführende Texte, in: Andersen, H. H., Henke, K. und J. G. v. d. Schulenburg (Hg.), Basiswissen Gesundheitsökonomie, Berlin: Edition Sigma, 41-62. 


\section{STAATLICHE ALLOKATIONSPOLITIK IM MARKTWIRTSCHAFTLICHEN SYSTEM}

Band 1 Horst Siebert (Hrsg.): Umweltallokation im Raum. 1982.

Band 2 Horst Siebert (Hrsg.): Global Environmental Resources. The Ozone Problem. 1982.

Band 3 Hans-Joachim Schulz: Steuerwirkungen in einem dynamischen Unternehmensmodell. Ein Beitrag zur Dynamisierung der Steuerüberwälzungsanalyse. 1981.

Band 4 Eberhard Wille (Hrsg.): Beitrăge zur gesamtwirtschaftlichen Allokation. Allokationsprobleme im intermediären Bereich zwischen offentlichem und privatem Wirtschaftssektor. 1983.

Band 5 Heinz König (Hrsg.): Ausbildung und Arbeitsmarkt. 1983.

Band 6 Horst Siebert (Hrsg.): Reaktionen auf Energiepreissteigerungen. 1982.

Band 7 Eberhard Wille (Hrsg.): Konzeptionelle Probleme öfentlicher Planung. 1983.

Band 8 Ingeborg Kiesewetter-Wrana: Exporterlösinstabilităt. Kritische Analyse eines entwicklungspolitischen Problems. 1982.

Band 9 Ferdinand Dudenhöfer: Mehrheitswahl-Entscheidungen über Umweltnutzungen. Eine Untersuchung von Gleichgewichtszustănden in einem mikroökonomischen Markt- und Abstimmungsmodell. 1983.

Band 10 Horst Siebert (Hrsg.): Intertemporale Allokation. 1984.

Band 11 Helmut Meder: Die intertemporale Allokation erschőpfbarer Naturressourcen bei fehlenden Zukunftsmărkten und institutionalisierten Marktsubstituten. 1984.

Band 12 Ulrich Ring: Öffentliche Planungsziele und staatliche Budgets. Zur Erfüllung offentlicher Aufgaben durch nicht-staatliche Entscheidungseinheiten. 1985.

Band 13 Ehrentraud Graw: Informationseffizienz von Terminkontraktmårkten für Währungen. Eine empirische Untersuchung. 1984.

Band 14 Rüdiger Pethig (Ed.): Public Goods and Public Allocation Policy. 1985.

Band 15 Eberhard Wille (Hrsg.): Öffentliche Planung auf Landesebene. Eine Analyse von Planungskonzepten in Deutschland, Österreich und der Schweiz. 1986.

Band 16 Helga Gebauer: Regionale Umweltnutzungen in der Zeit. Eine intertemporale Zwei-Regionen-Analyse. 1985.

Band 17 Christine Pfitzer: Integrierte Entwicklungsplanung als Allokationsinstrument auf Landesebene. Eine Analyse der öffentlichen Planung der Länder Hessen, Bayern und Niedersachsen. 1985.

Band 18 Heinz König (Hrsg.): Kontrolltheoretische Ansătze in makroökonometrischen Modellen. 1985.

Band 19 Theo Kempf: Theorie und Empirie betrieblicher Ausbildungsplatzangebote. 1985.

Band 20 Eberhard Wille (Hrsg.): Konkrete Probleme öffentlicher Planung. Grundlegende Aspekte der Zielbildung, Effizienz und Kontrolle. 1986.

Band 21 Eberhard Wille (Hrsg.): Informations- und Planungsprobleme in offentlichen Aufgabenbereichen. Aspekte der Zielbildung und Outputmessung unter besonderer Berūcksichtigung des Gesundheitswesens. 1986.

Band 22 Bernd Gutting: Der Einfluß der Besteuerung auf die Entwicklung der Wohnungs- und Baulandmärkte. Eine intertemporale Analyse der bundesdeutschen Steuergesetze. 1986.

Band 23 Heiner Kuhl: Umweltressourcen als Gegenstand internationaler Verhandlungen. Eine theoretische Transaktionskostenanalyse. 1987.

Band 24 Hubert Hornbach: Besteuerung, Inflation und Kapitalallokation. Intersektorale und internationale Aspekte. 1987. 
Band 25 Peter Müller: Intertemporale Wirkungen der Staatsverschuldung. 1987.

Band 26 Stefan Kronenberger: Die Investitionen im Rahmen der Staatsausgaben. 1988.

Band 27 Armin-Detlef Rieß: Optimale Auslandsverschuldung bei potentiellen Schuldendienstproblemen. 1988.

Band 28 Volker Ulrich: Preis- und Mengeneffekte im Gesundheitswesen. Eine Ausgabenanalyse von GKV-Behandlungsarten. 1988.

Band 29 Hans-Michael Geiger: Informational Efficiency in Speculative Markets. A Theoretical Investigation. Edited by Ehrentraud Graw. 1989.

Band 30 Karl Sputek: Zielgerichtete Ressourcenallokation. Ein Modellentwurf zur Effektivitätsanalyse praktischer Budgetplanung am Beispiel von Berlin (West). 1989.

\section{ALLOKATION IM MARKTWIRTSCHAFTLICHEN SYSTEM}

Band 31 Wolfgang Krader: Neuere Entwicklungen linearer latenter Kovarianzstrukturmodelle mit quantitativen und qualitativen Indikatorvariablen. Theorie und Anwendung auf ein mikroempirisches Modell des Preis-, Produktions- und Lageranpassungsverhaltens von deutschen und französischen Unternehmen des verarbeitenden Gewerbes. 1991.

Band 32 Manfred Erbsland: Die öffentlichen Personalausgaben. Eine empirische Analyse für die Bundesrepublik Deutschland. 1991.

Band 33 Walter Ried: Information und Nutzen der medizinischen Diagnostik. 1992.

Band 34 Anselm U. Römer: Was ist den Bürgern die Verminderung eines Risikos wert? Eine Anwendung des kontingenten Bewertungsansatzes auf das Giftmüllrisiko. 1993.

Band 35 Eberhard Wille, Angelika Mehnert, Jan Philipp Rohweder: Zum gesellschaftlichen Nutzen pharmazeutischer Innovationen. 1994.

Band 36 Peter Schmidt: Die Wahl des Rentenalters. Theoretische und empirische Analyse des Rentenzugangsverhaltens in West- und Ostdeutschland. 1995.

Band 37 Michael Ohmer: Die Grundlagen der Einkommensteuer. Gerechtigkeit und Effizienz. 1997.

Band 38 Evamaria Wagner: Risikomanagement rohstoffexportierender Entwicklungsländer. 1997.

Band 39 Matthias Meier: Das Sparverhalten der privaten Haushalte und der demographische Wandel: Makroökonomische Auswirkungen. Eine Simulation verschiedener Reformen der Rentenversicherung. 1997.

Band 40 Manfred Albring / Eberhard Wille (Hrsg.): Innovationen in der Arzneimitteltherapie. Definition, medizinische Umsetzung und Finanzierung. Bad Orber Gespräche über kontroverse Themen im Gesundheitswesen 25.-27.10.1996. 1997.

Band 41 Eberhard Wille / Manfred Albring (Hrsg.): Reformoptionen im Gesundheitswesen. Bad Orber Gespräche über kontroverse Themen im Gesundheitswesen 7.-8.11.1997. 1998.

Band 42 Manfred Albring / Eberhard Wille (Hrsg.): Szenarien im Gesundheitswesen. Bad Orber Gespräche über kontroverse Themen im Gesundheitswesen 5.-7.11.1998. 1999.

Band 43 Eberhard Wille / Manfred Albring (Hrsg.): Rationalisierungsreserven im deutschen Gesundheitswesen. 2000.

Band 44 Manfred Albring / Eberhard Wille (Hrsg.): Qualitätsorientierte Vergütungssysteme in der ambulanten und stationären Behandlung. 2001.

Band 45 Martin Pfaff / Dietmar Wassener / Astrid Sterzel / Thomas Neldner: Analyse potentieller Auswirkungen einer Ausweitung des Pharmaversandes in Deutschland. 2002.

Band 46 Eberhard Wille / Manfred Albring (Hrsg.): Konfliktfeld Arzneimittelversorgung. 2002. 
Band 47 Udo Schneider: Theorie und Empirie der Arzt-Patient-Beziehung. Zur Anwendung der Principal-Agent-Theorie auf die Gesundheitsnachfrage. 2002.

Band 48 Manfred Albring / Eberhard Wille: Die GKV zwischen Ausgabendynamik, Einnahmenschwäche und Koordinierungsproblemen. 2003.

Band 49 Uwe Jirjahn: X-Ineffizienz, Managementanreize und Produktmarktwettbewerb. 2004.

Band 50 Stefan Resch: Risikoselektion im Mitgliederwettbewerb der Gesetzlichen Krankenversicherung. 2004.

Band 51 Paul Marschall: Lebensstilwandel in Ostdeutschland. Gesundheitsőkonomische Implikationen. 2004.

Band 52 Eberhard Wille / Manfred Albring (Hrsg.): Paradigmenwechsel im Gesundheitswesen durch neue Versorgungsstrukturen? 8. Bad Orber Gesprăche. 6. - 8. November 2003. 2004.

Band 53 Eberhard Wille / Manfred Albring (Hrsg.): Versorgungsstrukturen und Finanzierungsoptionen auf dem Prüfstand. 9. Bad Orber Gesprăche. 11.-13. November 2004. 2005.

Band 54 Brit S. Schneider: Gesundheit und Bildung. Theorie und Empirie der Humankapitalinvestitionen. 2007.

www.peterlang.de 